Florida International University FIU Digital Commons

\title{
VHITS: Vertical Handoff Initiation and Target Selection in a Heterogeneous Wireless Network
}

Faisal Kaleem

Florida International University, kaleemf@fiu.edu

DOI: $10.25148 /$ etd.FI12050206

Follow this and additional works at: https://digitalcommons.fiu.edu/etd

\section{Recommended Citation}

Kaleem, Faisal, "VHITS: Vertical Handoff Initiation and Target Selection in a Heterogeneous Wireless Network" (2012). FIU

Electronic Theses and Dissertations. 602.

https://digitalcommons.fiu.edu/etd/602 


\section{FLORIDA INTERNATIONAL UNIVERSITY}

Miami, Florida

VHITS: VERTICAL HANDOFF INITIATION AND TARGET SELECTION IN A HETEROGENEOUS WIRELESS NETWORK

A dissertation submitted in partial fulfillment of the requirements for the degree of DOCTOR OF PHILOSOPHY in ELECTRICAL AND COMPUTER ENGINEERING by

Faisal Kaleem 
To: Dean Amir Mirmiran

College of Engineering and Computing

This dissertation, written by Faisal Kaleem and entitled VHITS: Vertical Handoff Initiation and Target Selection in a Heterogeneous Wireless Network, having been approved in respect to style and intellectual content, is referred to you for judgment.

We have read this dissertation and recommend that it be approved.

Jean H. Andrian

Hai Deng

Deng Pan

Kaushik Dutta

Abolfazl Mehbodniya

Kang K. Yen, Major Professor

Date of Defense: March 28, 2012

The dissertation of Faisal Kaleem is approved.

Dean Amir Mirmiran

College of Engineering and Computing

Dean Lakshmi N. Reddi

University Graduate School

Florida International University, 2012 


\section{DEDICATION}

I dedicate this dissertation to Hasan, Sameen, Maleeha, and to our parents, whose love, understanding, and sincere support gave me the inspiration to complete this overdue work. 


\section{ACKNOWLEDGMENTS}

I am grateful to Allah for providing me with the capability to finish this difficult task. My sincere gratitude goes to my advisor, Dr. Kang K. Yen, for his candid advice, support, and for helping me successfully finish my dissertation; thank you for believing in me. I would like to also acknowledge the guidance and moral support provided to me by Dr. Jean H. Andrian as he pushed me to complete this work. I greatly enjoyed his good humor and political talks. I cannot thank Dr. Abolfazl Mehbodniya enough, who in a very difficult time, and with short notice, held my hand and helped me succeed; thank you for your dedication, enormous help, and for the guidance you provided towards my dissertation. Thanks to Dr. Kaushik Dutta who agreed to attend my dissertation defense from Singapore, regardless of the immense time difference. Thanks are also due to Dr. Hai Deng and Dr. Deng Pan who agreed to become my dissertation committee members at the last moment. I cannot forget to thank Dr. Shekhar Bhansali, who supported and guided me since the day he took charge of the Department of Electrical and Computer Engineering at FIU; thanks for providing me all the opportunities to further my career.

I am also grateful to Dr. Christos Koulamas, Dr. Nicole Wishart, Dr. Manoel Oliveira, Dr. Zuzana Hlavacova Gurung, Ms. Maria Benincasa, Ms. Pat Brammer, Ms. Adelaida Ojedaa, Ms. Janey Sardinas, and Stefan Schins for their continuous support.

Finally, I would like to thank my wonderful wife Maleeha, and my parents, whose endless prayers and support helped me finish this tedious and challenging task. 


\section{ABSTRACT OF THE DISSERTATION \\ VHITS: VERTICAL HANDOFF INITIATION AND TARGET SELECTION IN A HETEROGENEOUS WIRELESS NETWORK}

by

Faisal Kaleem

Florida International University, 2012

Miami, Florida

\section{Professor Kang K. Yen, Major Professor}

Global connectivity, for anyone, at anyplace, at anytime, to provide high-speed, high-quality, and reliable communication channels for mobile devices, is now becoming a reality. The credit mainly goes to the recent technological advances in wireless communications comprised of a wide range of technologies, services, and applications to fulfill the particular needs of end-users in different deployment scenarios (Wi-Fi, WiMAX, and 3G/4G cellular systems). In such a heterogeneous wireless environment, one of the key ingredients to provide efficient ubiquitous computing with guaranteed quality and continuity of service is the design of intelligent handoff algorithms.

Traditional single-metric handoff decision algorithms, such as Received Signal Strength (RSS) based, are not efficient and intelligent enough to minimize the number of unnecessary handoffs, decision delays, and call-dropping and/or blocking probabilities.

This research presented a novel approach for the design and implementation of a multi-criteria vertical handoff algorithm for heterogeneous wireless networks. Several parallel Fuzzy Logic Controllers were utilized in combination with different types of ranking algorithms and metric weighting schemes to implement two major modules: the 
first module estimated the necessity of handoff, and the other module was developed to select the best network as the target of handoff.

Simulations based on different traffic classes, utilizing various types of wireless networks were carried out by implementing a wireless test-bed inspired by the concept of Rudimentary Network Emulator (RUNE).

Simulation results indicated that the proposed scheme provided better performance in terms of minimizing the unnecessary handoffs, call dropping, and call blocking and handoff blocking probabilities. When subjected to Conversational traffic and compared against the RSS-based reference algorithm, the proposed scheme, utilizing the FTOPSIS ranking algorithm, was able to reduce the average outage probability of MSs moving with high speeds by $17 \%$, new call blocking probability by $22 \%$, the handoff blocking probability by $16 \%$, and the average handoff rate by $40 \%$. The significant reduction in the resulted handoff rate provides MS with efficient power consumption, and more available battery life. These percentages indicated a higher probability of guaranteed session continuity and quality of the currently utilized service, resulting in higher user satisfaction levels. 


\section{TABLE OF CONTENTS}

CHAPTER

PAGE

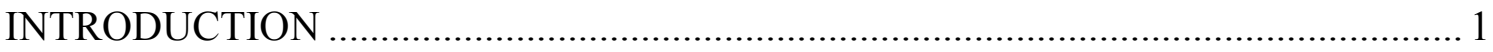

1.1 Motivation and Significance of Research .................................................... 4

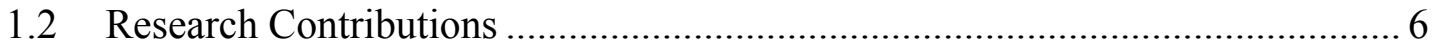

1.3 Justification of Using "AI" in Vertical Handoffs ........................................... 8

1.4 Organization of The Dissertation ................................................................ 10

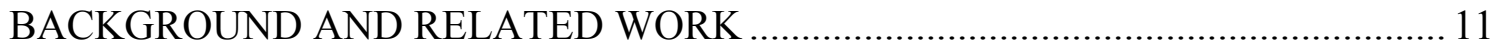

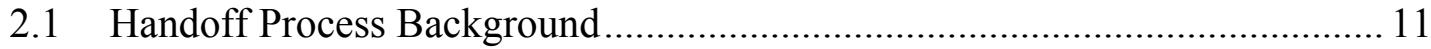

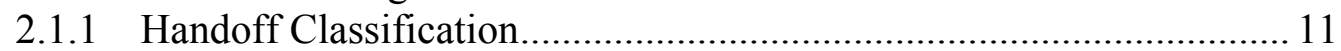

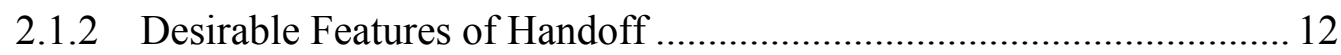

2.1.3 Vertical Handoff Process ............................................................... 13

2.1.4 Vertical Handoff Criteria and Metrics ................................................. 14

2.1.5 Classification of Vertical Handoff Algorithms .................................... 19

2.1.6 Performance Evaluation of Handoff Algorithms ................................ 22

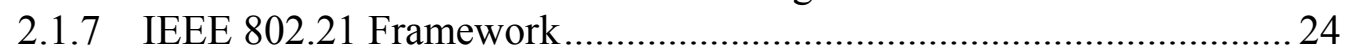

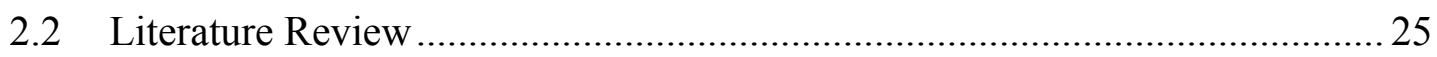

2.2.1 RSS Based Algorithms........................................................... 25

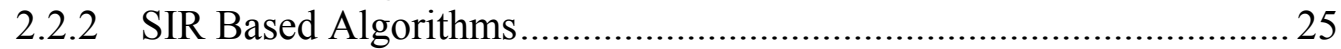

2.2.3 Velocity Based Algorithms ……………………................................ 25

2.2.4 Direction Biased Algorithms............................................................ 26

2.2.5 Minimum Power Algorithms ……………………......................... 26

2.2.6 User Preference Based Algorithms ................................................ 26

2.2.7 Context Aware Based Algorithms...................................................2 27

2.2.8 Cost Function Based Algorithms ………………………………….... 27

2.2.9 Multiple Criteria Based Algorithms ………………............................ 28

2.2.10 AI Based Approaches ......................................................................... 33

2.2.10.1 Fuzzy Logic Based Approaches............................................ 33

2.2.10.2 Artificial Neural Network Based Approaches ........................ 38

2.2.10.3 Combination Algorithms........................................................ 40

2.2.11 Brief Comparison of the Approaches ................................................... 45

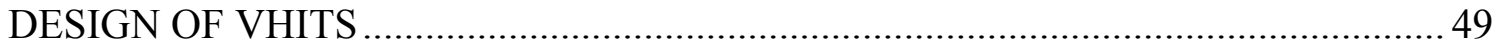

3.1 Comparison of VHITS with other Schemes ...................................................5 50

3.2 VHITS Handoff Necessity Estimation Module ………………………............5

3.2.1 System Attributes ........................................................................... 55

3.2.2 Weights Calculations for System Attributes .......................................58

3.2.2.1 Weights Calculations using AHP ...........................................59

3.2.2.2 Weights Calculations using FAHP............................................ 69

3.2.2.3 Weights Calculations using Linguistic Variables ................... 78

3.2.3 Brief Comparison of Weights Calculations Schemes ............................ 83

3.2.4 RSS Prediction Using Grey Prediction Theory ...................................... 83 
3.2.5 Normalization of Attributes using Fuzzy Techniques...........................86

3.2.6 Calculation of Degree of QoS for Current PoA ……………............... 89

3.2.7 VHO Factor Calculation using Fuzzy Logic ........................................ 91

3.2.7.1 Design of Fuzzy Logic Controller 1....................................... 91

3.2.7.2 Design of Fuzzy Logic Controller 2........................................ 96

3.2.7.3 Design of Fuzzy Logic Controller 3 ....................................... 98

3.2.7.4 Design of Fuzzy Logic Controller 4..................................... 101

3.2.8 Handoff Necessity Estimation.......................................................... 105

3.3 VHITS Target Network Selection Module ………….................................. 105

3.3.1 System Attributes .......................................................................... 107

3.3.2 Weights Calculations for System Attributes ....................................... 108

3.3.3 RSS Prediction Using Grey Prediction Theory ................................... 108

3.3.4 Normalization of Attributes using Fuzzy Techniques......................... 108

3.3.5 Significance Of Individual Parameters ............................................ 109

3.4 Network Selection (Ranking) Algorithms.................................................... 110

3.4.1 Network Selection using TOPSIS .................................................. 111

3.4.2 Network Selection using Fuzzy TOPSIS …………………………..... 113

3.4.3 Network Selection using Fuzzy VIKOR ………………………...... 121

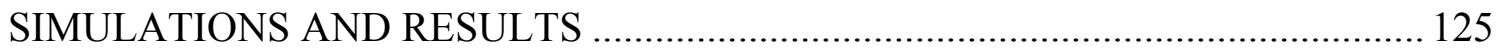

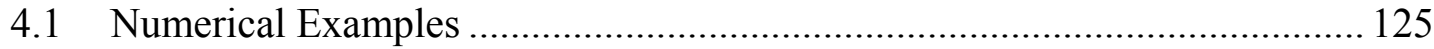

4.2 Simulation Setup and Environment......................................................... 142

4.3 Simulation Results: Single Mobile User ...................................................... 153

4.3.1 TOPSIS Based Network Selection .................................................. 154

4.3.2 FTOPSIS Based Network Selection................................................. 162

4.3.3 FVIKOR Based Network Selection ................................................ 166

4.4 Simulation Results: Single Mobile User Preferences ..................................... 168

4.4.1 TOPSIS Based Network Ranking with User Preferred Cost .............. 168

4.4.2 TOPSIS Network Ranking with User Preferred Security ................... 171

4.4.3 FTOPSIS and FVIKOR Rankings with User Preferred Cost.............. 173

4.4.4 FTOPSIS And FVIKOR Rankings with User Preferred Security ...... 175

4.5 Simulation Results: Multiple Mobile Users ................................................... 176

4.5.1 Average Outage Probability with TOPSIS Based Selection.............. 179

4.5.2 Average Outage Probability with FTOPSIS and FVIKOR ................. 182

4.5.3 Average Handoff Blocking Probability Based on TOPSIS ................ 183

4.5.4 Average Handoff Blocking Probability, FTOPSIS and FVIKOR ..... 186

4.5.5 Average New Call Blocking Probability based on TOPSIS .............. 188

4.5.6 Average New Call Blocking Probability, FTOPSIS and FVIKOR ... 190

4.5.7 Average Handoff Rate Based on TOPSIS.......................................... 191

4.5.8 Average Handoff Rate Based On FTOPSIS and FVIKOR ................. 193

4.5.9 Percentage Of Network Connections -TOPSIS ................................ 194

4.5.10 Percentage Of Network Connection-FTOPSIS and FVIKOR ....... 202

4.6 Comparison between Different Schemes ...................................................... 203 
CONCLUSIONS AND FUTURE WORK

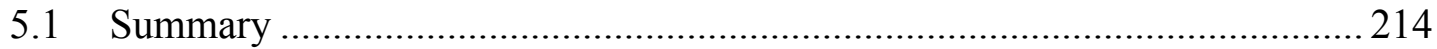

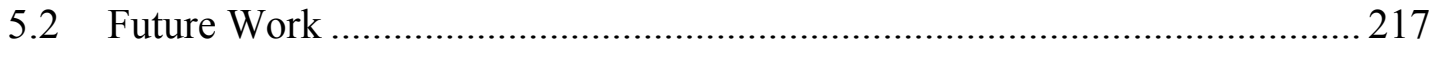

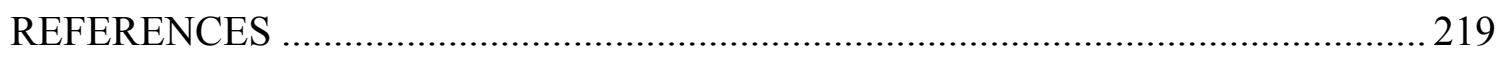

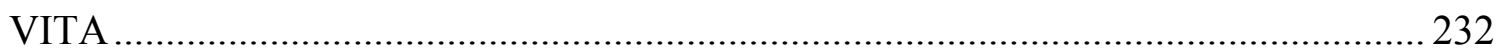




\section{LIST OF TABLES}

TABLE

PAGE

Table 2.1: Overall Comparison of Handoff Approaches ............................................ 47

Table 2.2: The Comparison of Handoff Algorithms ................................................ 48

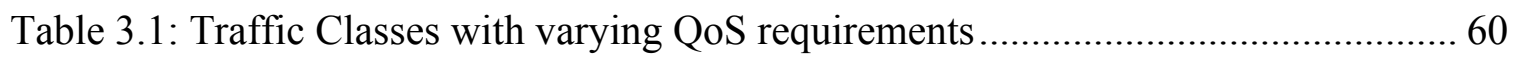

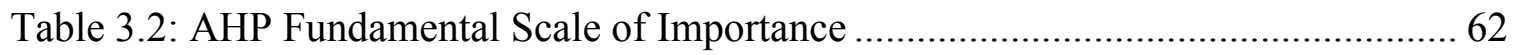

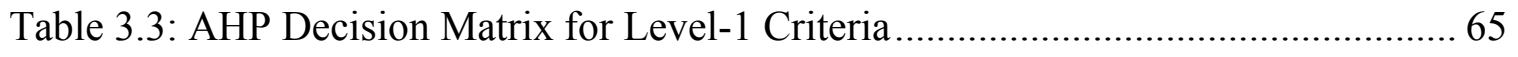

Table 3.4: AHP Decision Matrix for QoS Sub-criteria for Conversational Traffic.......... 65

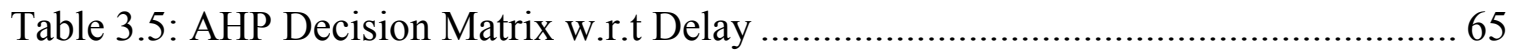

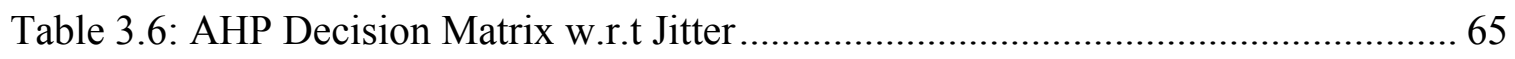

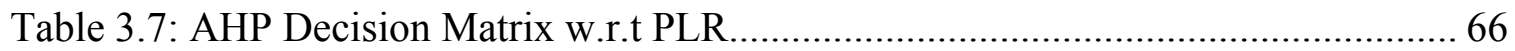

Table 3.8: AHP Interdependence Matrix for QoS parameters...................................... 66

Table 3.9: AHP Decision Matrix for QoS Sub-criteria for Interactive Traffic................. 67

Table 3.10: AHP Decision Matrix for QoS Sub-criteria for Background Traffic ............ 68

Table 3.11: AHP Decision Matrix for QoS Sub-criteria for Streaming Traffic ............... 69

Table 3.12: TFNs and Reciprocal TFNs for FAHP Levels of Importance ..................... 73

Table 3.13: FAHP Decision Matrix for Leve-1 Criteria for Conversational Traffic........ 74

Table 3.14: FAHP Decision Matrix for QoS Sub-criteria for Conversational Traffic ..... 74

Table 3.15: FAHP Decision Matrix w.r.t Delay .................................................... 74

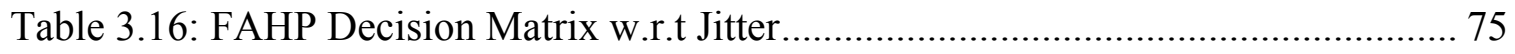

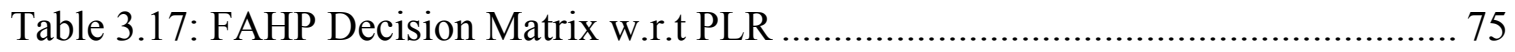

Table 3.18: FAHP Interdependence Matrix for QoS parameters …............................ 75

Table 3.19: FAHP Decision Matrix for QoS Sub-criteria for Interactive Traffic ............ 76 
Table 3.20: FAHP Decision Matrix for QoS Sub-criteria for Background traffic 77

Table 3.21: FAHP Decision Matrix for QoS Sub-criteria for Streaming traffic .............. 77

Table 3.22: Linguistic Variables with TFNs and Crisp Values .................................... 80

Table 3.23: Linguistic Variables and Weights for Level-1 Criteria .............................. 80

Table 3.24: Linguistic Variables and Weights for QoS criteria, Conversational traffic... 80

Table 3.25: Linguistic Variables and Weights w.r.t Delay ........................................ 81

Table 3.26: Linguistic Variables and Weights w.r.t Jitter ....................................... 81

Table 3.27: Linguistic Variables and Weights w.r.t PLR .......................................... 81

Table 3.28: Linguistic Variables Interdependence Matrix for QoS parameters .............. 81

Table 3.29: Linguistic Variables \& Weights for QoS Sub-criteria, Interactive traffic..... 81

Table 3.30: Linguistic Variables \& Weights for QoS Sub-criteria, Background traffic .. 82

Table 3.31: Linguistic Variables \& Weights for QoS criteria, Streaming traffic ............. 83

Table 3.32: Comparison of Different Weighting Schemes ........................................ 84

Table 3.33: Predicted Values of RSS using GPT for Different Network Types ............. 86

Table 3.34: Parameter Ranges for Different Network Types .................................... 88

Table 4.1: Different Parameters for Current PoA ................................................... 126

Table 4.2: New Parameter Set When User Walks Away from Current PoA ................ 130

Table 4.3: Parameter Set for Available Networks in-Range of Walking User .............. 131

Table 4.4: Normalized Parameter Set and AHP based Weights for All Networks......... 132

Table 4.5: Fuzzy Decision Matrix for FTOPSIS ...................................................... 145

Table 4.6: Linguistic Weights used with FTOPSIS for Different Traffic Classes ......... 145

Table 4.7: Normalized weighted Matrix, Streaming Class, showing FPIS and FNIS ... 146

Table 4.8: FTOPSIS Calculations for All Traffic Classes ........................................ 146 
Table 4.9: FVIKOR Decision Matrix, showing FBV and FWV, Streaming traffic

Table 4.10: FVIKOR Calculations for All Traffic Classes......................................... 148

Table 4.11: Network Parameters used for VHITS Simulation ................................... 153

Table 4.12: System Parameters used for VHITS Simulation .................................... 154

Table 4.13: VHITS Selection Schemes Comparison for Conversational Traffic ........... 210

Table 4.14: VHITS Selection Schemes Comparison for Streaming Traffic................... 211

Table 4.15: VHITS Selection Schemes Comparison for Background Traffic................ 212

Table 4.16: VHITS Selection Schemes Comparison for Interactive Traffic .................. 213 


\section{LIST OF FIGURES}

FIGURE

PAGE

Figure 1.1: Evolution of Wireless Communications (from $1 \mathrm{G}$ to $4 \mathrm{G}$ ) ............................ 3

Figure 1.2: Handoff Management Concept ............................................................. 3

Figure 2.1: Horizontal and Vertical Handoff in Heterogeneous Wireless Networks ....... 15

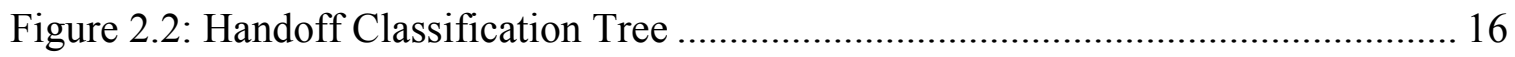

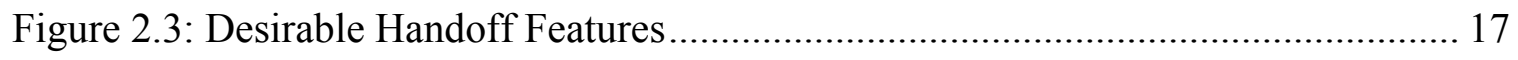

Figure 2.4: Metrics used in Vertical Handoff Decisions .......................................... 17

Figure 2.5: Traffic Classes and Handoff Metrics..................................................... 23

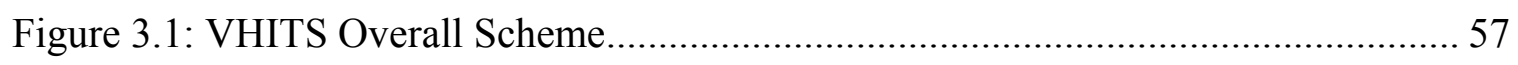

Figure 3.2: VHITS Handoff Necessity Estimation Module.......................................... 58

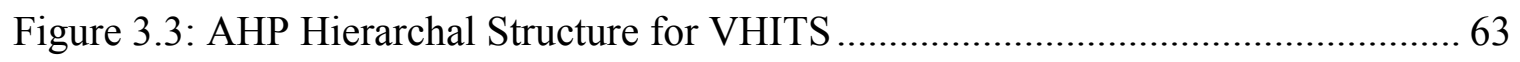

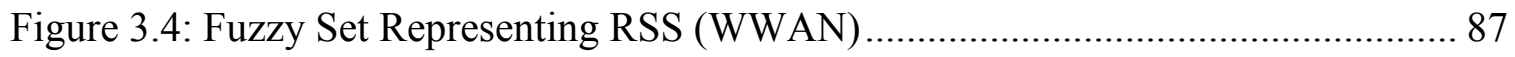

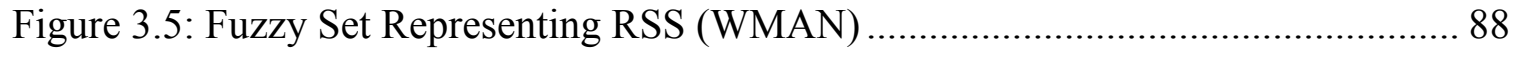

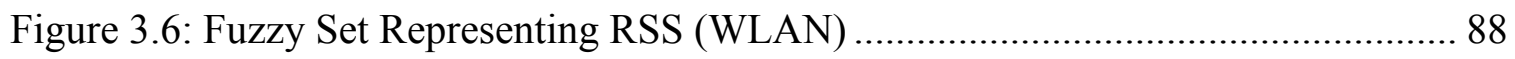

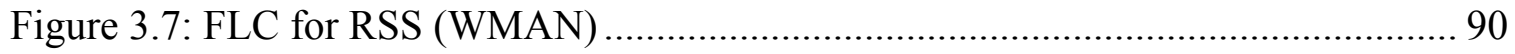

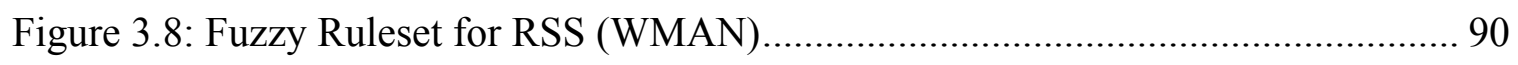

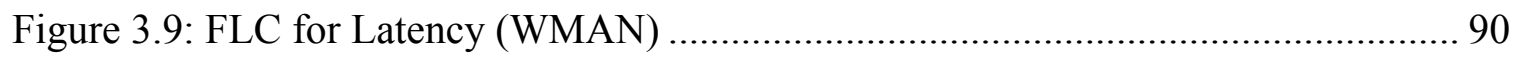

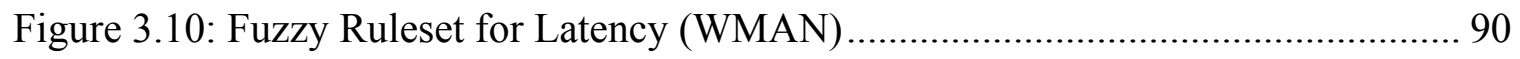

Figure 3.11: Fuzzy Logic Controllers for VHO Necessity Estimation.......................... 92

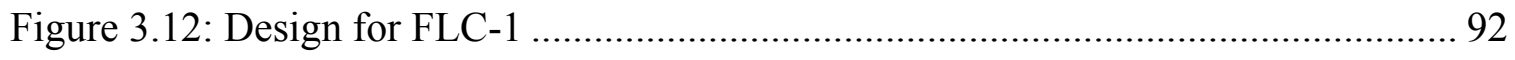

Figure 3.13: Membership Function for MS-Velocity for FLC-1 ............................... 93

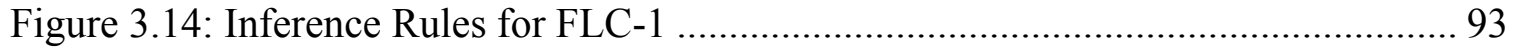


Figure 3.15: The Control Surface of the Output Variable WWAN-Reject for FLC-1 .... 94

Figure 3.16: Rules Evaluation for FLC-1 …......................................................... 94

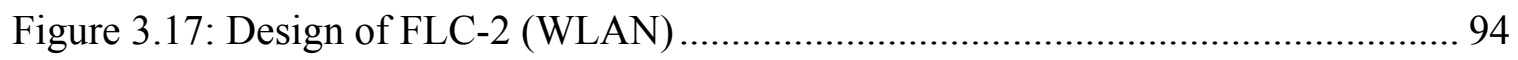

Figure 3.18: Membership Functions for FLC-2 (WLAN) ........................................ 95

Figure 3.19: Membership Functions for FLC-2 (WMAN) ....................................... 95

Figure 3.20: Membership Functions for FLC-2 (WWAN) ....................................... 95

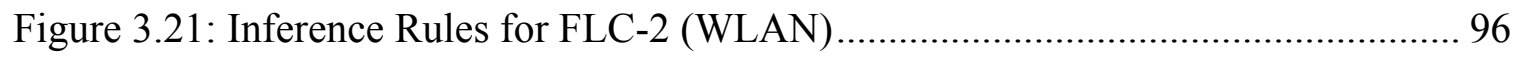

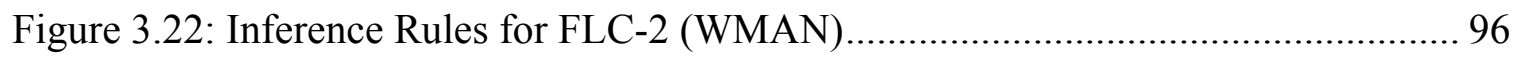

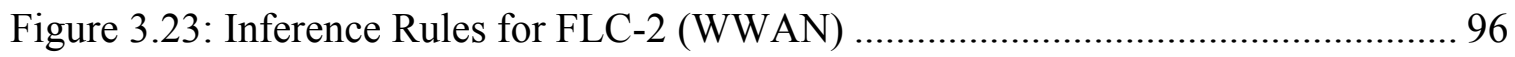

Figure 3.24: The Control Surface of the Output Variable WMAN-Reject for FLC-2 _.... 97

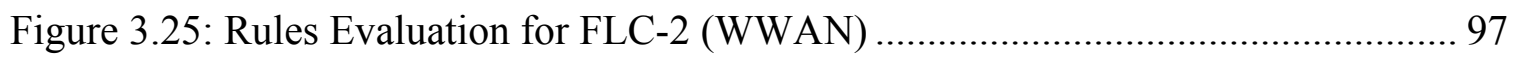

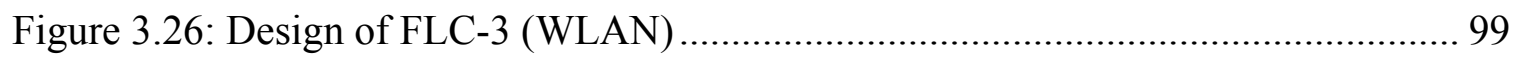

Figure 3.27: Membership Function for Input Variable PRSS of FLC-3 (WLAN).......... 99

Figure 3.28: Membership Function for Input Variable QoS of FLC-3 (WLAN)........... 99

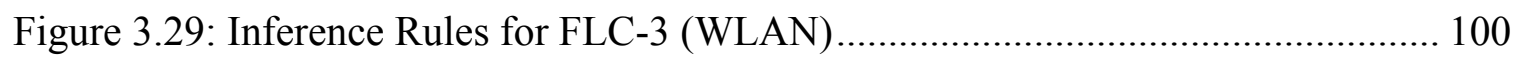

Figure 3.30: Control Surface of the Variable PRSS-QoS-Factor of FLC-3 (WLAN).... 100

Figure 3.31: Rules Evaluation for FLC-3 (WLAN) (Low RSS and High QoS)............ 100

Figure 3.32: Rules Evaluation for FLC-3 (WLAN) (High RSS and Low QoS)............ 101

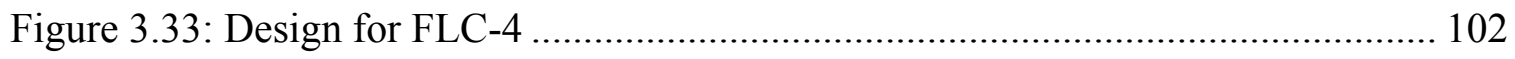

Figure 3.34: Membership function for MS-PoA-Distance-Factor for FLC-4 .............. 102

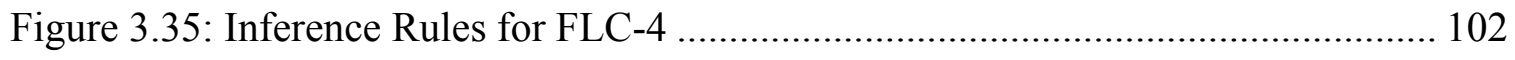

Figure 3.36: Control Surface for the Variable WLAN-HO-Factor for FLC-4 ….......... 103

Figure 3.37: Control Surface for the Variable WMAN-HO-Factor for FLC-4 ............. 103 
Figure 3.38: Control Surface for the Variable WWAN-HO-Factor for FLC-4. 103

Figure 3.39: Rules Evaluation for FLC-4 (High Handoff Probability) 104

Figure 3.40: Rules Evaluation for FLC-4 (Low Handoff Probability) .......................... 104

Figure 3.41: VHITS Target Network Selection Scheme …...................................... 107

Figure 4.1: First-Level Weights based on All Weighting Schemes............................ 127

Figure 4.2: AHP-based Weights for QoS Parameters.............................................. 128

Figure 4.3: FAHP-based Weights for QoS Parameters ......................................... 128

Figure 4.4: Linguistic-Variables based Weights for QoS Parameters ......................... 129

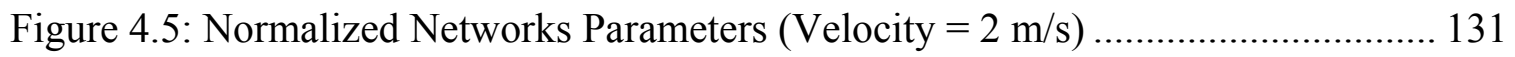

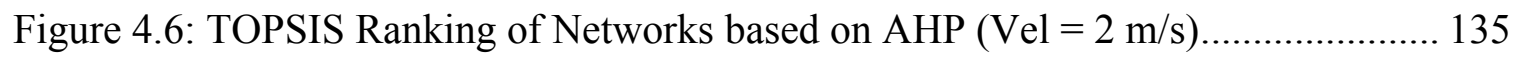

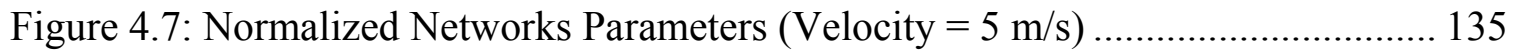

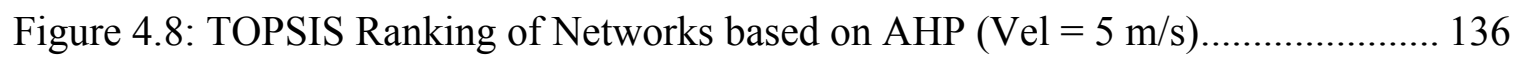

Figure 4.9: TOPSIS Ranking of Networks based on AHP $(\mathrm{Vel}=10 \mathrm{~m} / \mathrm{s}) \ldots \ldots \ldots \ldots \ldots \ldots \ldots \ldots \ldots \ldots . . . .136$

Figure 4.10: TOPSIS Ranking of Networks based on FAHP $(\mathrm{Vel}=2 \mathrm{~m} / \mathrm{s}) \ldots \ldots \ldots \ldots \ldots . . . . . .137$

Figure 4.11: TOPSIS Ranking of Networks based on FAHP $(\mathrm{Vel}=5 \mathrm{~m} / \mathrm{s}) \ldots \ldots \ldots \ldots \ldots \ldots . . . \ldots 137$

Figure 4.12: TOPSIS Ranking of Networks based on FAHP $(\mathrm{Vel}=10 \mathrm{~m} / \mathrm{s}) \ldots \ldots \ldots \ldots \ldots . . . \ldots 138$

Figure 4.13: TOPSIS Ranking of Networks based on TFN $(\mathrm{Vel}=2 \mathrm{~m} / \mathrm{s}) \ldots \ldots \ldots \ldots \ldots \ldots \ldots \ldots \ldots \ldots . . . . .138$

Figure 4.14: TOPSIS Ranking of Networks based on TFN weighting $(\mathrm{Vel}=5 \mathrm{~m} / \mathrm{s}) \ldots .139$

Figure 4.15: TOPSIS Ranking of Networks based on TFN (Vel $=10 \mathrm{~m} / \mathrm{s}) \quad \ldots \ldots \ldots \ldots \ldots . . . . .139$

Figure 4.16: TOPSIS Ranking with AHP $(\mathrm{Vel}=5 \mathrm{~m} / \mathrm{s}, \mathrm{WWAN}-$ Loading $=100 \%) \ldots . . .141$

Figure 4.17: TOPSIS with AHP (Vel $=5 \mathrm{~m} / \mathrm{s}$, WWAN, WMAN-Loading $=100 \%) \ldots 141$

Figure 4.18: FTOPSIS Ranking for Available Networks (Velocity $=5 \mathrm{~m} / \mathrm{s}$ ).............. 144

Figure 4.19: FVIKOR Network Rankings for all Traffic classes (Velocity $=5 \mathrm{~m} / \mathrm{s}) \ldots . .144$ 
Figure 4.20: System Model for VHITS Simulation...................................................... 151

Figure 4.21: System model (zoomed-in) Depicting the Mobility of Single User........... 151

Figure 4.22: Distribution of MSs across Different Networks......................................... 152

Figure 4.23: Percentage of NW-Connection, TOPSIS, AHP, for Conversational ......... 155

Figure 4.24: Percentage of NW-Connection, TOPSIS, AHP, for Interactive.................. 156

Figure 4.25: Percentage of NW-Connection, TOPSIS, AHP, for Background ............... 156

Figure 4.26: Percentage of NW-Connection, TOPSIS, AHP, for Streaming ................. 157

Figure 4.27: Percentage of NW-Connection, TOPSIS, FAHP, for Background ............. 158

Figure 4.28: Percentage of NW-Connection, TOPSIS, FAHP, for Conversational ....... 158

Figure 4.29: Percentage of NW-Connection, TOPSIS, FAHP, for Interactive .............. 159

Figure 4.30: Percentage of NW-Connection, TOPSIS, FAHP, for Streaming ................ 159

Figure 4.31: Percentage of NW-Connection, TOPSIS, TFN, for Background............... 160

Figure 4.32: Percentage of NW-Connection, TOPSIS, TFN, for Conversational .......... 160

Figure 4.33:Percentage of NW-Connection, TOPSIS, TFN, for Interactive .................. 161

Figure 4.34: Percentage of NW-Connection, TOPSIS, TFN, for Streaming.................. 161

Figure 4.35: Percentage of NW-Connection, FTOPSIS for Background Traffic........... 164

Figure 4.36: Percentage of NW-Connection, FTOPSIS for Conversational traffic ....... 164

Figure 4.37: Percentage of NW-Connection, FTOPSIS for Interactive Traffic ............. 165

Figure 4.38: Percentage of NW-Connection, FTOPSIS for Streaming Traffic .............. 165

Figure 4.39: Percentage of NW-Connection, FVIKOR for Background Traffic ........... 167

Figure 4.40: Percentage of NW-Connection, FVIKOR for Conversational Traffic....... 167

Figure 4.41: Percentage of NW-Connection, FVIKOR for Interactive Traffic.............. 169

Figure 4.42: Percentage of NW-Connection, FVIKOR for Streaming Traffic............... 169 
Figure 4.43: Percentage Connections, Preferred Cost, TOPSIS, AHP, Streaming ........ 170

Figure 4.44: Percentage Connections, Preferred Cost, TOPSIS, FAHP, Streaming ...... 170

Figure 4.45: Percentage Connection, Preferred Cost, TOPSIS, TFN, Streaming ......... 171

Figure 4.46: Percentage of NW, Preferred Security, TOPSIS, AHP, Streaming ........... 172

Figure 4.47: Percentage of NW, Preferred Security, TOPSIS, FAHP, Streaming ......... 172

Figure 4.48: Percentage of NW, Preferred Security, TOPSIS, TFN, Streaming ............ 173

Figure 4.49: Percentage of NW, Preferred Cost, FTOPSIS, Streaming $\quad$.............. 174

Figure 4.50: Percentage of NW, Preferred Cost, FVIKOR, Streaming ......................... 174

Figure 4.51: Percentage of NW, Preferred Security, FTOPSIS, Streaming .................. 175

Figure 4.52: Percentage of NW, Preferred Security, FVIKOR, Streaming ................... 176

Figure 4.53: Average Outage Probability, TOPSIS, AHP, Conversational................... 180

Figure 4.54: Average Outage Probability, TOPSIS, FAHP, Conversational ................ 181

Figure 4.55: Average Outage Probability, TOPSIS, TFN, Conversational ................... 181

Figure 4.56: Average Outage Probability, FTOPSIS, Conversational ........................ 182

Figure 4.57: Average Outage Probability, FVIKOR, Conversational .......................... 183

Figure 4.58: Average Handoff Blocking Probability based on TOPSIS-AHP .............. 184

Figure 4.59: Average Handoff Blocking Probability based on TOPSIS-FAHP............ 185

Figure 4.60: Average Handoff Blocking Probability based on TOPSIS-TFN .............. 185

Figure 4.61: Average Handoff Blocking Probability based on FTOPSIS .................... 187

Figure 4.62: Average Handoff Blocking Probability based on FVIKOR..................... 187

Figure 4.63: Average New Call Blocking Probability based on TOPSIS-AHP ............ 188

Figure 4.64: Average New Call Blocking Probability based on TOPSIS-FAHP.......... 189

Figure 4.65: Average New Call Blocking Probability based on TOPSIS-TFN ............ 189 
Figure 4.66: Average New Call Blocking Probability based on FTOPSIS 190

Figure 4.67: Average New Call Blocking Probability based on FVIKOR 191

Figure 4.68: Average Handoff Rate based on TOPSIS-AHP 192

Figure 4.69: Average Handoff Rate based on TOPSIS-FAHP..... 192

Figure 4.70: Average Handoff Rate based on TOPSIS-TFN 193

Figure 4.71: Average Handoff Rate based on FTOPSIS 195

Figure 4.72: Average Handoff Rate based on FVIKOR. 196

Figure 4.73: Percentage of NW per Average Calls, TOPSIS-AHP, Vel= $1 \mathrm{~m} / \mathrm{s}$ 196

Figure 4.74: Percentage of NW per Average Calls, TOPSIS-AHP, Vel $=5 \mathrm{~m} / \mathrm{s}$ 198

Figure 4.75: Percentage of NW per Average Calls, TOPSIS-AHP, Vel $=9 \mathrm{~m} / \mathrm{s}$ 199

Figure 4.76: Percentage of NW per Average Calls, TOPSIS-FAHP, Vel $=1 \mathrm{~m} / \mathrm{s}$ 199

Figure 4.77: Percentage of NW per Average Calls, TOPSIS-FAHP, Vel $=5 \mathrm{~m} / \mathrm{s} \ldots \ldots . .200$

Figure 4.78: Percentage of NW per Average Calls, TOPSIS-FAHP, Vel $=9 \mathrm{~m} / \mathrm{s} \ldots \ldots \ldots . .200$

Figure 4.79: Percentage of NW per Average Calls, TOPSIS-TFN, Vel= $1 \mathrm{~m} / \mathrm{s} \quad \ldots \ldots \ldots . . .201$

Figure 4.80: Percentage of NW per Average Calls, TOPSIS-TFN, Vel $=5 \mathrm{~m} / \mathrm{s} \ldots \ldots \ldots \ldots . . .201$

Figure 4.81: Percentage of NW per Average Calls, TOPSIS-TFN, Vel $=9 \mathrm{~m} / \mathrm{s} \ldots \ldots \ldots . . .202$

Figure 4.82: Percentage of NW per Average Calls, FTOPSIS, Vel $=1$ m/s ............... 204

Figure 4.83: Percentage of NW per Average Calls, FTOPSIS, Vel $=5$ m/s ................ 204

Figure 4.84: Percentage of NW per Average Calls, FTOPSIS, Vel $=9 \mathrm{~m} / \mathrm{s} \ldots \ldots \ldots \ldots \ldots . . . .205$

Figure 4.85: Percentage of NW per Average Calls, FVIKOR, Vel $=1 \mathrm{~m} / \mathrm{s} \ldots \ldots \ldots \ldots \ldots . . .205$

Figure 4.86: Percentage of NW per Average Calls, FVIKOR, Vel $=5 \mathrm{~m} / \mathrm{s} \ldots \ldots \ldots \ldots \ldots . . .206$

Figure 4.87: Percentage of NW per Average Calls, FVIKOR, Vel $=9 \mathrm{~m} / \mathrm{s} \ldots \ldots \ldots \ldots \ldots \ldots . . . . .206$ 


\section{LIST OF ABBREVIATIONS}

3G: Third Generation

4G: Fourth Generation

ABC: Always Best Connected

AGO: Accumulated Generating Operation

AHP: Analytical Hierarchy Process

AI: Artificial Intelligence

AN: Access Network

AP: Access Point

ANFIS: Adaptive Neuro-Fuzzy based Inference System

ANN: Artificial Neural Network

BER: Bit Error Rate

BS: Base Station

CDMA: Code Division Multiple Access

CINR: Channel to Interference plus Noise Ratio

EDGE: Enhanced Data for GSM Evolution

ELECTRE: Elimination and Choice Translating Priority

FAHP: Fuzzy Analytical Hierarchy Process

FIS: Fuzzy Inference System

FLC: Fuzzy Logic Controller

FLS: Fuzzy Logic System

FNQD: Fuzzy Normalized Quantitative Decision

FQDA: Fuzzy Quantitative Decision Algorithm 
FTOPSIS: Fuzzy TOPSIS

FVIKOR: Fuzzy VIKOR

GA: Genetic Algorithm

GERAN: GSM EDGE Radio Access Network

GPRS: General Packet Radio Service

GPT: Grey Prediction Theory

GRA: Grey Relational Analysis

GRC: Grey Relational Coefficient

GSM: Global System for Mobile Communications

HAPS: High Altitude Platform Cellular system

HSDPA/HSUPA: High Speed Downlink/Uplink Packet Access

JRRM: Joint Radio Resource Management

MADM: Multi Attribute Decision Making

MCDM: Multi Criteria Decision Making

MODM: Multiple Objective Decision Making

MEW: Multiplicative Exponent Weighting

MLP: Multi-Layer Perceptron

MS: Mobile Station

MUSIC: MUltiple SIgnal Classification

PAN: Personal Area Network

PBNN: Probabilistic Neural Network

PEV: Performance Evaluation Value

PLR: Packet Loss Ratio 
PoA: Point of Attachment

PNN: Probabilistic Neural Network

PRSS: Predicted RSS

QoS: Quality of Service

QDV: Quantitative Decision Value

RBFN: Radial-Basis Function Network

RRM: Radio Resource Management

RSS: Received Signal Strength

RUNE: Rudimentary Network Emulator

RSSI: Received Signal Strength Intensity

SAW: Simple Additive Weighting

SIR: Signal to Interference Ratio

SINR: Signal to Interference plus Noise Ratio

SMART: Simple Multi-Attribute Rate Technique

TFN: Triangular Fuzzy Number

TOPSIS: Techniques for Order Preference by Similarity to Ideal Solution

UMTS: Universal Mobile Telecommunication Systems

UoD: Universe of Discourse

VANET: Vehicular Ad-hoc Network

VHITS: Vertical Handoff Initiation and Target Selection

VHO: Vertical Handoff

VIKOR: \{Serbian: VIseKriterijumsa Optimizacija I Kompromisno Resenje, meaning: multi-criteria optimization and compromise solution $\}$ 
VoIP: Voice over IP

Wi-Fi: Wireless Fidelity

WiMAX: Worldwide Interoperability for Microwave Access

WLAN: Wireless Local Area Network

WMAN: Wireless Metropolitan Area Network

WWAN: Wireless Wide Area Network 


\section{CHAPTER 1}

\section{INTRODUCTION}

Over the past few years, there have been several exciting innovations in wireless network technology [1]. As can be seen from Figure 1.1, the current trends and demands in the area of wireless communications are to deliver real-time multimedia applications over heterogeneous wireless networks with guaranteed Quality of Service (QoS). The consumer demand, to access such applications and services anywhere and anytime, is continuously on the rise. New technological developments, such as the Fourth Generation (4G) wireless systems [2, 3] and their integration, offer these rich services and applications at high data transfer rates and allow for global roaming and seamless mobility over a diverse range of heterogeneous wireless networks [4-6].

Mobile Stations (MSs) in a typical 4G network will be equipped with multiple interfaces, and will have the required intelligence to make improved decisions to be able to connect to a variety of Access Networks (ANs) in order to provide rich multimedia services. These access networks include different types of cellular networks such as Code Division Multiple Access (CDMA), Global System for Mobile Communication (GSM), High Speed Downlink/Uplink Packet Access (HSDPA/HSUPA), General Packet Radio Services (GPRS) [7, 8], Bluetooth-based Personal Area Network (PAN) [9], IEEE 802.11 Wireless Local Area Network (WLAN) [10], IEEE 802.16 Worldwide Interoperability for Microwave Access (WiMAX) [11], Vehicular Ad-hoc Network (VANET), and Satellite networks. These wireless networks often have overlapping coverage in the same service areas and can offer innovative services based on user demands. The ultimate goal of such an environment is to provide simple, uninterrupted accesses to any type of 
desired service at any time, independent of devices, locations, and available networks, [12] while also maintaining satisfactory user experience in a cost-efficient manner.

The wireless technologies in a heterogeneous wireless network are usually different from each other from a technological point of view. Most of them usually differ in terms of, but not limited to, their offered bandwidths, operating frequencies and costs, coverage areas, and latencies. Currently, no single wireless technology claims to provide cost-effective services, which offers high bandwidths and low latencies to all mobile users in a large coverage area. This is where the need for well-organized vertical handoffs (VHOs) between heterogeneous wireless technologies becomes evident.

The term "handoff", or "handover" [13], refers to the process of transferring a mobile station from one base station or channel to another. One example is a seamless transfer of an ongoing voice or video conversation from one channel served by a core network to another. More clearly, handoff is the process of changing communication channel (frequency, data rate, modulation scheme, spreading code, or their combination) associated with the current connection, while, a communication session (or call) is in progress.

A handoff process can be thought of as having two major stages: handoff initiation and handoff execution [13]. In the first phase, a decision is made regarding the selection of the new Base Station (BS), or Access Point (AP), to which the MS will be transferred. In the execution phase, new radio links are formed between the BS/AP and MS, and resources are allocated. A comprehensive overview of handoff management is provided in [14] and depicted in Figure 1.2. 


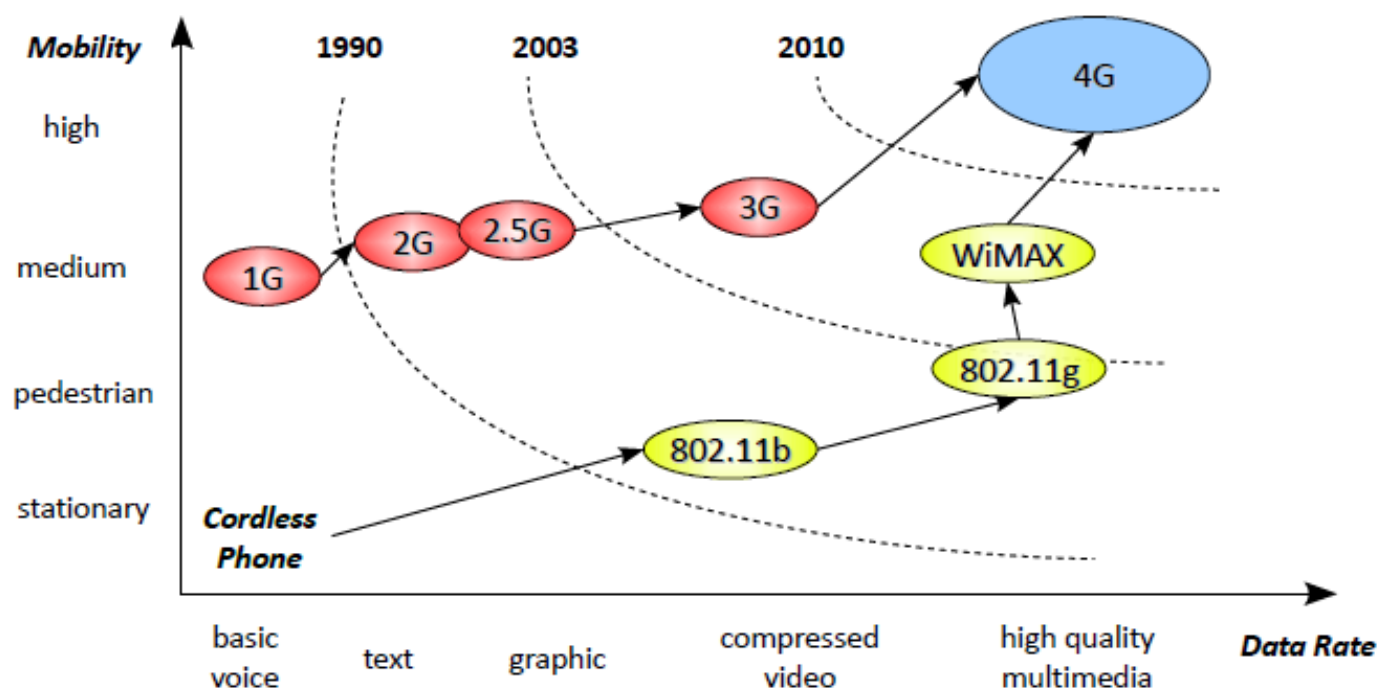

Figure 1.1: Evolution of Wireless Communications (from $1 \mathrm{G}$ to $4 \mathrm{G}$ )

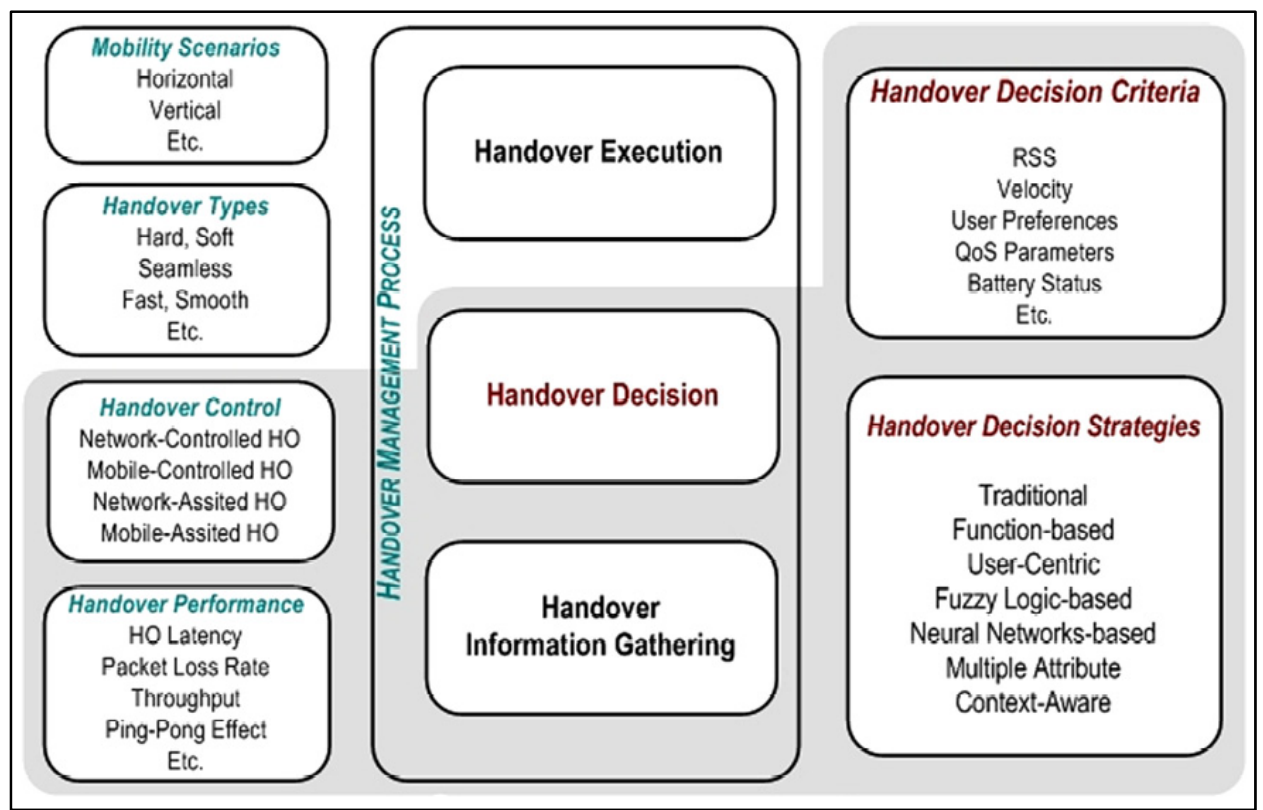

Figure 1.2: Handoff Management Concept [14]

This chapter begins with Section 1.1 describing the motivation and significance of this research work, followed by Section 1.2, providing a quick overview of related research contributions. Section 1.3 explains how techniques, based on Artificial Intelligence (AI), can be used to perform handoff necessity estimation and target network 
selection in a more intelligent and efficient manner. Finally, Section 1.4 provides an outline of the dissertation.

\subsection{MOTIVATION AND SIGNIFICANCE OF RESEARCH}

So far, significant research has been done to achieve seamless mobility while an MS moves across different heterogeneous wireless networks. However, this research mainly focuses on an important aspect of seamless mobility: vertical handoff initiations and decisions. Horizontal handoff decisions between homogeneous wireless networks are made mainly on the basis of Received Signal Strength (RSS), whereas decisions for vertical handoffs are typically performed based on more than one network's parameters, including, but not limited to, RSS, MS-Velocity, Security, Cost, and QoS parameters. These decisions often incorporate network-operators' policies and end-users' preferences

as well. Automating these handoff decisions in a heterogeneous environment is a complex task compared to homogeneous wireless networks due to several reasons including varying network characteristics, environmental conditions, and mobility patterns.

Many of the existing handoff algorithms, which are based on single metric such as RSS, do not exploit the benefits of multi-criteria and the inherent knowledge about the sensitivities of these handoff parameters in a heterogeneous wireless environment. In addition, while performing vertical handoffs, these algorithms do not take into account the QoS of an ongoing session to maximize the end-users' satisfaction based on their preferences, location and/or application contexts. Factors like available network bandwidth, latency, security, usage cost, power consumption, battery status of MS, and 
user preferences should be thoroughly considered while performing these handoff decisions.

In nearly all the existing multi-criteria handoff schemes, assigning different weights helps prioritize network parameters. Most of the time, the assignment of these weights is done manually without considering how much of a weight is needed for a certain network parameter. This could lead to a degraded handoff performance if one parameter is given higher weight value as compared to another, especially during an ongoing user-session, such as a Voice over IP (VoIP) conversations, where achieving a minimum level of quality of service is essential. Thus, calculation for the correct weights for network parameters is an important task when operating in a heterogeneous wireless environment. Furthermore, nearly all handoff schemes utilize crisp values for these weights, ignoring the fact that typical values of parameters in a wireless network are not precise and are characterized by inherent uncertainty. Therefore, in order to guarantee the quality of the currently utilized service, proper weight assignment, especially for QoSrelated parameters, is of utmost importance and should be done very carefully. In addition, the fuzzy nature of these values should be kept in mind while assigning these weights.

The handoff can be divided into handoff initiation and target network selection. Most of the research work deals with the target network selection, ignoring the handoff initiation and necessity estimation that is of equal importance, as handoff initiation and its necessity estimation play a critical role in maximizing end-user's satisfaction. Just like the values of weights associated with network parameters, the calculations to perform handoff necessity estimation and target network selection are usually done using raw or 
crisp values of parameters provided by different networks, without paying much attention to the vagueness and fuzziness contained in this information.

\subsection{RESEARCH CONTRIBUTIONS}

In this research work, an intelligent, scalable, and flexible hybrid scheme is proposed to perform intelligen and efficient handoff necessity estimations and target network selection decisions. The proposed scheme is divided into two parts. The first part examines the existing conditions of current PoA to estimate the necessity of handoff using Fuzzy Logic (FL). In the second stage, different parameters of all available candidate networks are utilized to determine a new PoA, or an access network, that can best fulfill the end-user's requirements. The target network selection scheme utilizes certain ranking algorithms to rank the available networks based on multiple criteria.

The proposed scheme intends to maximize the end-user's satisfaction, taking into account the quality of the currently utilized service that the end-user experiences at the mobile terminal. The following are the main contributions of this research work:

- A comprehensive survey of current network selection algorithms in heterogeneous wireless networks is provided.

- A module based on Grey Prediction Theory (GPT) is designed to predict the future values of the measured RSS in order to minimize the call dropping probability of the MS due to a sudden drop of RSS common in wireless networks.

- Several parallel Fuzzy Logic Controllers (FLCs) are developed to estimate the necessity of handoffs. The necessity estimation module is important, as 
a handoff that is done too early is costly in terms of valuable network resources. Similarly, a handoff that is performed too late increases the call-dropping probability of MS. Furthermore, parallel FLCs offer reduced rule-sets, are more efficient, and less complex than a single FLC containing bulky rule set.

- Since a heterogeneous wireless network is comprised of many different wireless technologies with varying parameter ranges, the proposed scheme also utilizes FLCs to normalize the incoming data to a common scale. Consequently, benefit-type parameters such as RSS, and cost-type parameters such as network latency, measured from different networks, are normalized using FLCs.

- Different weight calculation methods are developed to calculate priority weights for network parameters. These methods include Analytic Hierarchy Process (AHP), Fuzzy AHP (FAHP), and Linguistic Variables and Triangular Fuzzy Numbers (TFNs). FAHP and Linguistic Variables are utilized to demonstrate the weight calculations, keeping in mind the vagueness and fuzziness of these subjective values.

- Several Multi-Attribute Decision Making (MADM) ranking algorithms are utilized to demonstrate the selection of best target network among other available candidate networks based on the measured values of different parameters from each network. These include algorithms such as Techniques for Order Preference by Similarity to Ideal Solution (TOPSIS), Fuzzy extension of TOPSIS (FTOPSIS), and Fuzzy extension 
of VIKOR (Serbian: VIseKriterijumsa Optimizacija I Kompromisno Resenje, i.e.: multi-criteria optimization and compromise solution) (FVIKOR).

- A simulation test-bed for a heterogeneous wireless network is created for evaluating the performance of the proposed scheme. Simulation is carried out for three different types of networks providing four different types of services. This test-bed simulates in detail a real heterogeneous wireless environment with all the Radio Resource Management (RRM) modules such as channel assignment, mobility and propagation modules, etc.

To the best of our knowledge, this research work is a first attempt to utilize FAHP, TFNs and Linguistic Variables to calculate weights for network attributes and to apply FTOPSIS and FVIKOR to perform target network selection in the context of a heterogeneous wireless environment. It is also important to mention that this research work attempts to distinguish between the different types of traffic classes based on the QoS parameters and tries to find an improved weighting schemes based on these types.

\subsection{JUSTIFICATION OF USING “AI” IN VERTICAL HANDOFFS}

Fuzzy logic and Artificial Neural Networks (ANNs) are extensively used in literature to perform vertical handoff decisions in order to select the best access network for an MS. The application of these complicated algorithms is necessitated by the complexity of vertical handoff decisions and dynamic conditions of wireless networks. Unlike the traditional vertical handoff decision approaches where the decisions are solely 
based on RSS, fuzzy logic and ANN-based approaches can combine various design parameters to perform such decisions, which are inherently optimized.

The fact that fuzzy logic can mimic human expert reasoning and that many of the terms used to describe a signal (weak, far, strong, close) are fuzzy in nature, makes fuzzy logic a strong candidate for performing vertical handoff decisions. Fuzzy logic can adapt easily to these decisions as it can overcome radio environment uncertainty, fluctuations, and can deal with heterogeneous inter-system parameters (shadowing effect, traffic variations, etc.).

Artificial neural networks on the other hand, have the ability to handle large sets of data in an environment that demands fast processing. They can interpret complex data and learn typical trends from it. The learned system can then be used to predict the response of an arbitrary input signal. Hence, ANN can be trained to predict an MS's handoff to the best available access networks.

Another possibility exists where a hybrid solution of fuzzy logic and neural networks can be utilized to perform vertical handoff decisions. Known sensitivities of handoff parameters are used to create a Fuzzy Logic System (FLS) rule base, but this demands large storage and high computational complexity. These demands can be circumvented by replacing the rule base in FLS by an ANN to derive an adaptive algorithm that retains the high performance of FLS and provides an efficient architecture for storage and computational requirements. An ANN can be trained to learn complex relationships among the multiple inputs and output of a handoff system. After the ANN is trained, its parameters can be used to predict or estimate the outputs for given inputs.

An overview of these handoff schemes will be presented in Chapter 2. 


\subsection{ORGANIZATION OF THE DISSERTATION}

The organization of this dissertation is as follows. Chapter 2 provides a brief background on the process of handoff, followed by a comprehensive overview of the related work in the area of vertical handoff decisions. In Chapter 3, an overall framework of the proposed handoff scheme is presented. Simulation and experimental results are

presented in Chapter 4, and finally, Chapter 5 concludes this research work with suggested future research directions. 


\section{CHAPTER 2}

\section{BACKGROUND AND RELATED WORK}

This chapter begins by providing a background related to the handoff process followed by a comprehensive survey of different approaches to make vertical handoff decisions. Through the literature review, the available handoff algorithms can be grouped into different categories based on the main handoff decision criterion used [14]: RSSbased, multiple-criteria decision based, user-preference based, context-aware approaches, cost-function based, and AI based approaches. Since this research is focused on the usage of AI based approaches, a detailed overview of the related work will be provided mainly for this category while briefly mentioning the others. Sections 2.2.1-2.2.8 briefly categorize different handoff decision algorithms. In Section 2.2.9, a brief overview of multiple criteria based algorithms, is provided. Section 2.2 .10 provides a detailed overview of the related works using the AI based approaches. Finally, in Section 2.2.11, a comparison among few of these techniques is provided.

\subsection{HANDOFF PROCESS BACKGROUND}

\subsubsection{HANDOFF CLASSIFICATION}

Handoffs can be classified in several ways as discussed below:

Horizontal and Vertical Handoff: Depending on the type of network technologies involved, handoff can be classified as either horizontal or vertical [15]. Traditional handoff, also called horizontal or intra-system handoff, occurs when the MS switches between different BSs or APs of the same access network. For example, this typically happens when the user moves between two geographically adjacent cells of a third 
generation $(3 \mathrm{G})$ cellular network. On the other hand, vertical handoff or inter-system handoff involves two different network-interfaces representing different wireless access networks or technologies, e.g., BS in IEEE 802.16 and an AP in IEEE 802.11. Figure 2.1 [16] depicts the two types of handoffs in heterogeneous wireless networks where horizontal handoff occurs between two WLANs, and vertical handoff occurs between a WLAN and a CDMA network.

Hard and Soft Handoff: This classification of handoff depends upon the number of BSs and/or APs to which an MS is associated with at any given moment. Hard handoff, also called "break before make", involves only one BS or AP at a time. The MS must break its connection from the current access network before it can connect to a new one. In a soft handoff, also called "make before break", an MS can communicate and connect with more than one access network during the handoff process [15, 17].

Mobile-controlled, Mobile-assisted, and Network-controlled Handoff: As the names suggest, these types of handoff classifications are based on the entity, MS or access network, which make the handoff decisions [18]. Mobile-assisted handoff is the hybrid of mobile-controlled and network-controlled handoff where the MS makes the handoff decisions in cooperation with the access network. A detailed handoff classification tree can be found in [18] and is shown in Figure 2.2.

\subsubsection{DESIRABLE FEATURES OF HANDOFF}

Figure 2.3 [18] describes several desirable features of handoff algorithms as mentioned in the literature $[18,19]$. Some of these features are described below: 
- Speed: Handoff should be fast enough to avoid service degradation and/or interruption at the MS. Mobility of an MS at a high speed requires the handoff to be done promptly.

- Reliability: Handoff should be reliable such that the MS will be able to maintain the required QoS after handoff.

- Successful: Free channels and resources must be available at the target access network in order to make the handoff successful.

- Number of Handoffs: The number of handoffs must be minimized. Excessive number of handoffs results in poor QoS and excessive processing overheads as well as power loss, which is a critical issue in MSs with limited battery power.

- Multiple Criteria Handoffs: The target access network should be intelligently chosen based on multiple criteria. Identification of a correct AN prevents unnecessary and frequent handoffs.

\subsubsection{VERTICAL HANDOFF PROCESS}

The traditional horizontal handoff research involves handoff decisions based on the manual evaluation of RSS measured at the MS to support the "Always Best Connected" communications. These traditional handoffs are triggered when the RSS value of the serving BS falls below a specified threshold. On the other hand, an MS in a heterogeneous wireless environment can move between different ANs with different functionality and characteristics (bandwidth, latency, power consumption, cost, etc.) which cannot be directly compared. Hence, in case of vertical handoffs, RSS itself is not 
sufficient for making efficient and intelligent handoff decisions; other system metrics including, but not limited to, cost, network-load and performance, available bandwidth, security, and user preferences should be taken into consideration as well. On the other hand, the inclusion of multiple metrics increases the complexity of vertical handoff decisions and makes the entire process more challenging. A vertical handoff comprises of three phases as follows [20]:

Network Discovery: An MS with multiple active interfaces can discover several wireless networks based on broadcasted service advertisements from these wireless networks. However, keeping all these interfaces active all the time can significantly affect the battery power of the MS.

Handoff Triggering and Decision: This is the phase where the decision regarding "when" to perform handoff is made. In this phase, the target wireless access network is selected based on multiple criteria, as discussed before.

Handoff Execution: This is the last phase of the vertical handoff process where the actual transfer of the current session to the new AN takes place. This requires the current network to transfer routing and other contextual information related to the MS to the newly selected AN as quickly as possible.

\subsubsection{VERTICAL HANDOFF CRITERIA AND METRICS}

Figure 2.4 [18] and Figure 2.5 [21] describe different traffic classes and several handoff metrics that are used as inputs to the various vertical handoff algorithms. These metrics are described below: 


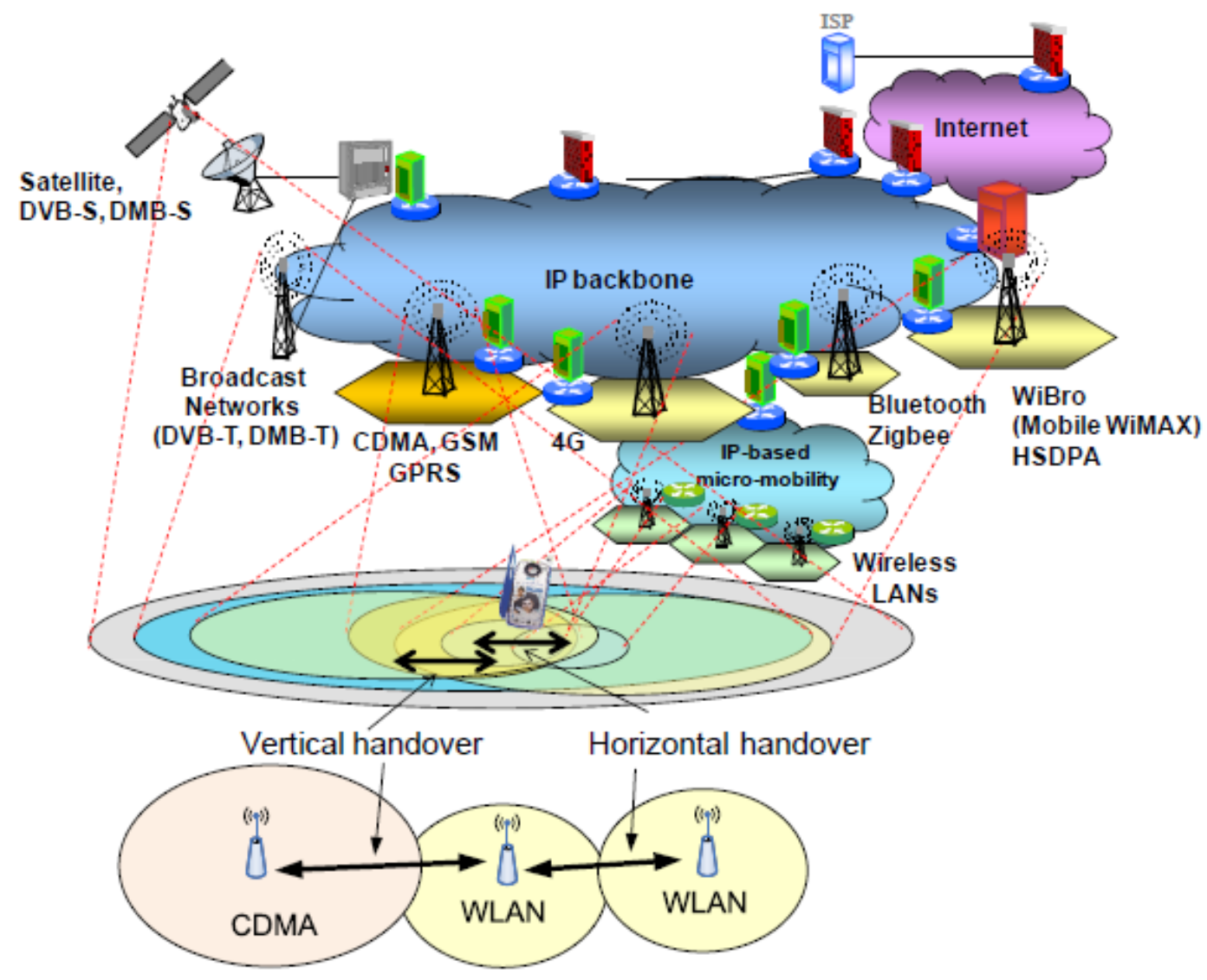

Figure 2.1: Horizontal and Vertical Handoff in Heterogeneous Wireless Networks [16]

- Received Signal Strength: This criterion is simple, direct, and widely used in both horizontal and vertical handoffs. This network metric is easy to measure and is directly relevant to the QoS of an application. Also, RSS readings are inversely proportional to the distance between the MS and the $\mathrm{BS}$, and could result in excessive and/or unnecessary handoffs.

- Available Bandwidth: Measured in bits/sec (bps), available bandwidth is used to determine traffic-loading conditions of an AN, and is a good measure of available communication resources at the BS. 


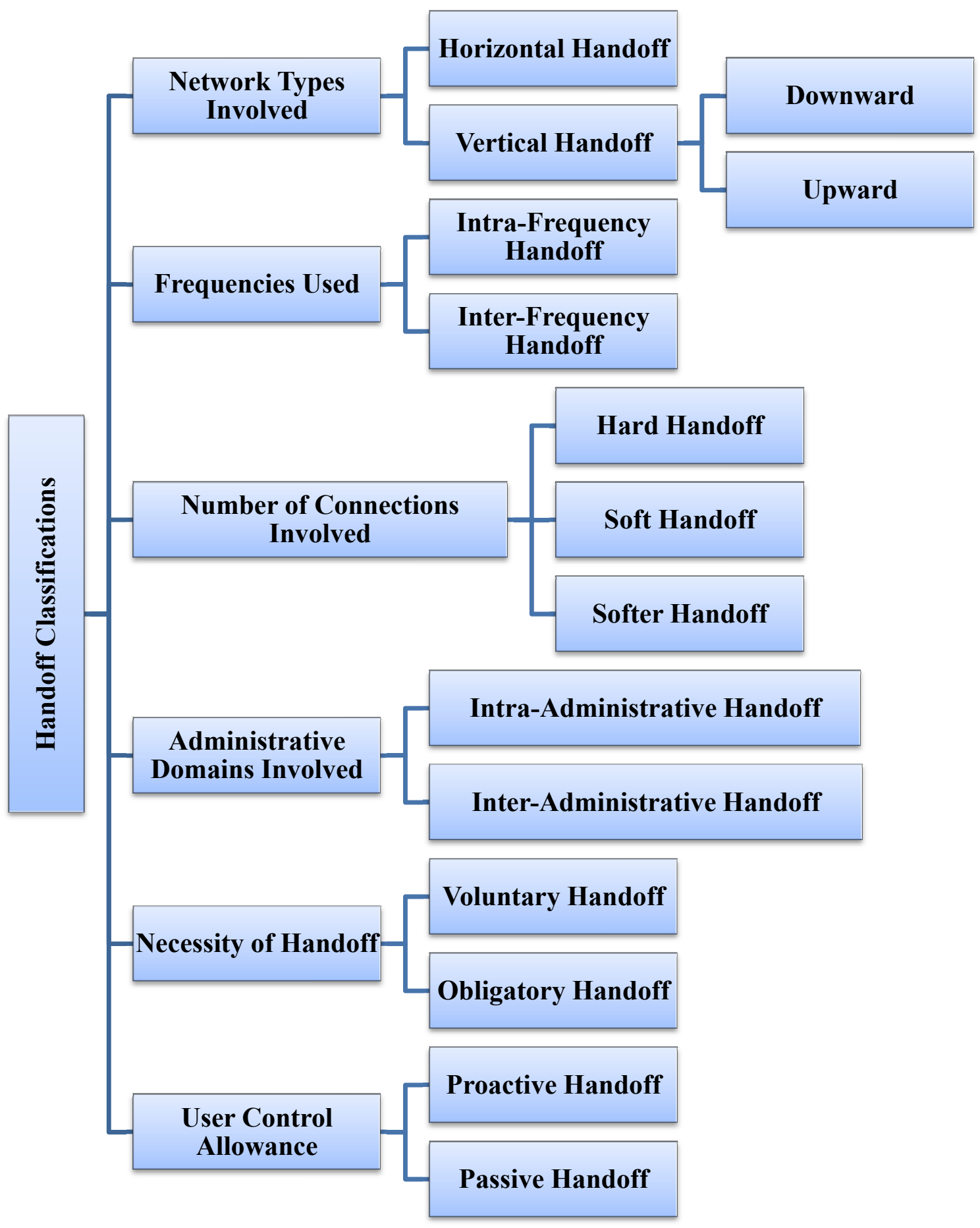

Figure 2.2: Handoff Classification Tree 


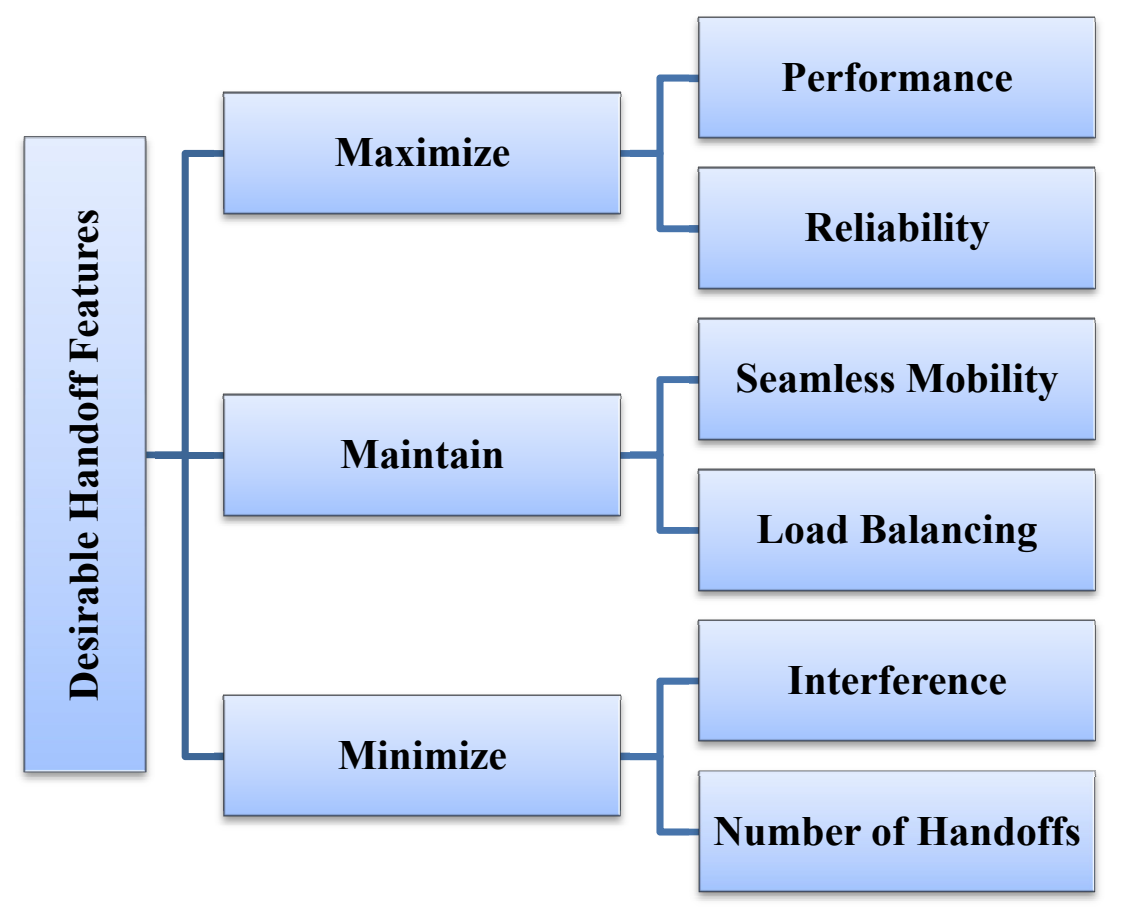

Figure 2.3: Desirable Handoff Features

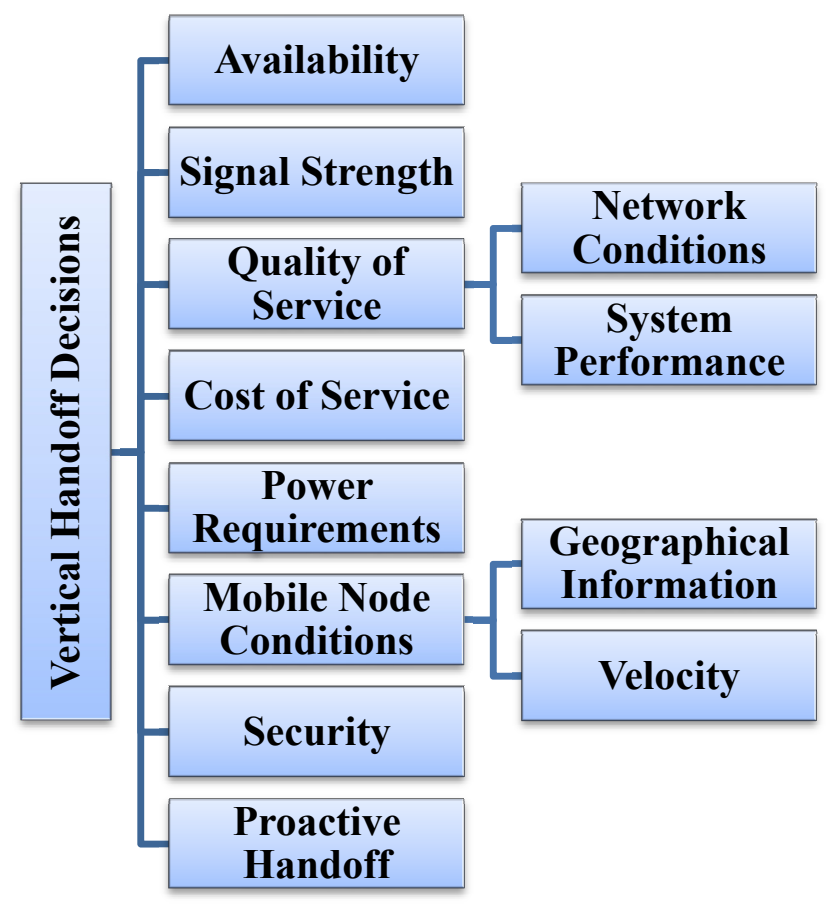

Figure 2.4: Metrics used in Vertical Handoff Decisions 
- Network Connection Duration: This is the amount of time that the MS remains connected to a specific AN. This time duration depends on the location and velocity of the MS, which in turn affect its RSS. Due to different coverage areas in heterogeneous wireless networks, the evaluation of this criterion is very important to determine two factors: 1) the triggering conditions required for the handoff at the right time in order to maintain a satisfactory QoS while avoiding wastage of network resources and 2) to reduce the number of unnecessary handoffs. For example, a hasty handoff from an IEEE 802.11 WLAN to a 3G cellular network would result in network resources being wasted. On the other hand, delaying the handoffs between these networks would result in handoff failures and subsequent call drops. Statistics, such as total time spent in an AN and arrival time of a new call in the network, can also be used as handoff criteria.

- Monetary Cost: Different operators may operate heterogeneous wireless networks and may have varying costs associated with them. The network with the least cost should be a preferred target of handoff.

- Handoff Latency: For an MS, handoff latency is defined as the elapsed time between the last packet received from the old $\mathrm{AN}$, and the arrival of the first packet via the new AN after a successful handoff. This metric varies considerably between various heterogeneous wireless technologies.

- Security: Certain applications require that the confidentiality, and/or the integrity of the transferred data be preserved. This metric can be used to 
handoff to a network that offers higher security as compared to other available networks.

- Power Consumption: Handoff process demands a fair amount of power consumption. If an MS were running low on battery power, it would be preferable to handoff to a target AN that would help extend the MS's battery life [18].

- Velocity: Velocity is an important decision factor as it relates to the network-connection-duration metric and location of the MS. An MS travelling at a very high speed may result in excessive handoffs between wireless networks.

\subsubsection{CLASSIFICATION OF VERTICAL HANDOFF ALGORITHMS}

Handoff algorithms can be classified based on handoff criteria and their processing. In the following paragraphs, we will categorize these algorithms. A detailed literature review of these categories is presented in Section 2.2.

- Signal Strength Based Algorithms: The RSS is used as the main criterion to perform handoffs [1]. Different variations of this category exist as follows:

- Relative Signal Strength Algorithms: This is based on the comparison of RSS of the current AN with that of the target network. The advantage of this algorithm is the ability of the MS to connect and remain connected with the BS that has the strongest RSS. However, excessive handoffs are possible due to shadow variations associated with the RSS. A variation of this algorithm incorporates hysteresis [22], where a handoff is initiated 
only if the RSS measured from the target BS exceeds the RSS from the current BS by an amount of hysteresis. Hysteresis reduces the number of unnecessary handoffs but can also introduce delay and prevent required and necessary handoffs that could result in call drops.

- Absolute Signal Strength Algorithms: In this type of algorithm, a handoff is requested as soon as the measured RSS drops below a certain threshold level. For a noise and interference limited system, the typical threshold values in a cellular system are $-100 \mathrm{dbm}$ and $-95 \mathrm{dbm}$, respectively [23]. Varying these threshold values may result in better handoffs. The disadvantage of this category of algorithms is the setting of the thresholds based on the RSS. When RSS is high due to interference, a desirable and necessary handoff will not take place. Similarly, a low value of RSS will result in unnecessary handoffs regardless of the signal QoS. A variation of this scheme is to utilize multiple thresholds to initiate and execute handoffs.

For vertical handoffs, relative RSS is not applicable. This is due to the fact that RSSs from different wireless technologies in a heterogeneous environment cannot be directly compared against each other.

- Combined Absolute and Relative Signal Strength Algorithms: These algorithms must satisfy two conditions to perform handoffs [24]: The average RSS of the current BS must fall below an absolute threshold value and the average RSS of the target BS must exceed the average RSS of the current BS by an amount of hysteresis. 
- Bandwidth Based Algorithms: The available bandwidth offered by the AN to the MS is used as the main criterion for these algorithms [1]. Handoff can also be initiated based on both criteria, i.e., available bandwidth and RSS $[1,25]$.

- Distance Based Algorithms: In this category, the MS is always connected to the nearest BS. Propagation delays from different networks are compared for relative distance measurement.

- Signal to Interference Ratio (SIR) Based Algorithms: SIR is a measure of communication quality. These algorithms trigger the handoff when the SIR of the current BS drops below a certain threshold and another BS is available to provide a better SIR. Just like relative-RSS based algorithms, hysteresis can be incorporated into these algorithms as well.

- Velocity Adaptive and Direction Biased Algorithms: Handoff requests from fast moving MSs must be processed quickly and with minimal delays. Handoffs to the BSs, towards which an MS is moving, are encouraged in this category of algorithms. A variation of this is a pre-selection direction biased algorithm [26].

- Cost Function Based Algorithms: In this category, several performance metrics (usage cost, available bandwidth, delay, security offerings and power consumption, etc.) are used to calculate an overall network cost for all available candidate networks. Handoff is then performed to the network with the smallest calculated cost [27]. Weights and biases may be used with different performance metrics to simulate different network conditions and user preferences. 
- Pattern Recognition Based Algorithms: These types of algorithms can identify meaningful regularities in noisy or uncertain environments. The handoff problem is studied as a pattern recognition problem in $[28,29]$.

- Prediction Based Algorithms: Estimated future values of different handoff metrics are used to predict the behavior of the system [30].

- Fuzzy Logic and Neural Network Based Algorithms: These algorithms are suitable for multi-criteria handoffs. Sometimes, it is not possible to develop the analytical model for the handoff process, especially when a richer set of input parameters are involved. This is where machine-learning techniques are used to model the complex handoff process.

Fuzzy logic based techniques allow us to model the qualitative aspects of human experts' knowledge and reasoning behind the handoff process to be encoded as handoff algorithms [31]. On the other hand, Neural Networks, using a comprehensive set of inputs and desired output(s), can be trained to perform optimal handoff decisions [32].

A third possibility is where adaptive versions of the above mentioned algorithms can be created where the system can monitor its performance and modify its internal structure to create highly effective and optimal handoff decisions [33].

\subsubsection{PERFORMANCE EVALUATION OF HANDOFF ALGORITHMS}

The performance of different vertical handoff algorithms can be evaluated and compared by measuring the following metrics: 


\section{Vertical Handoff Decisions}

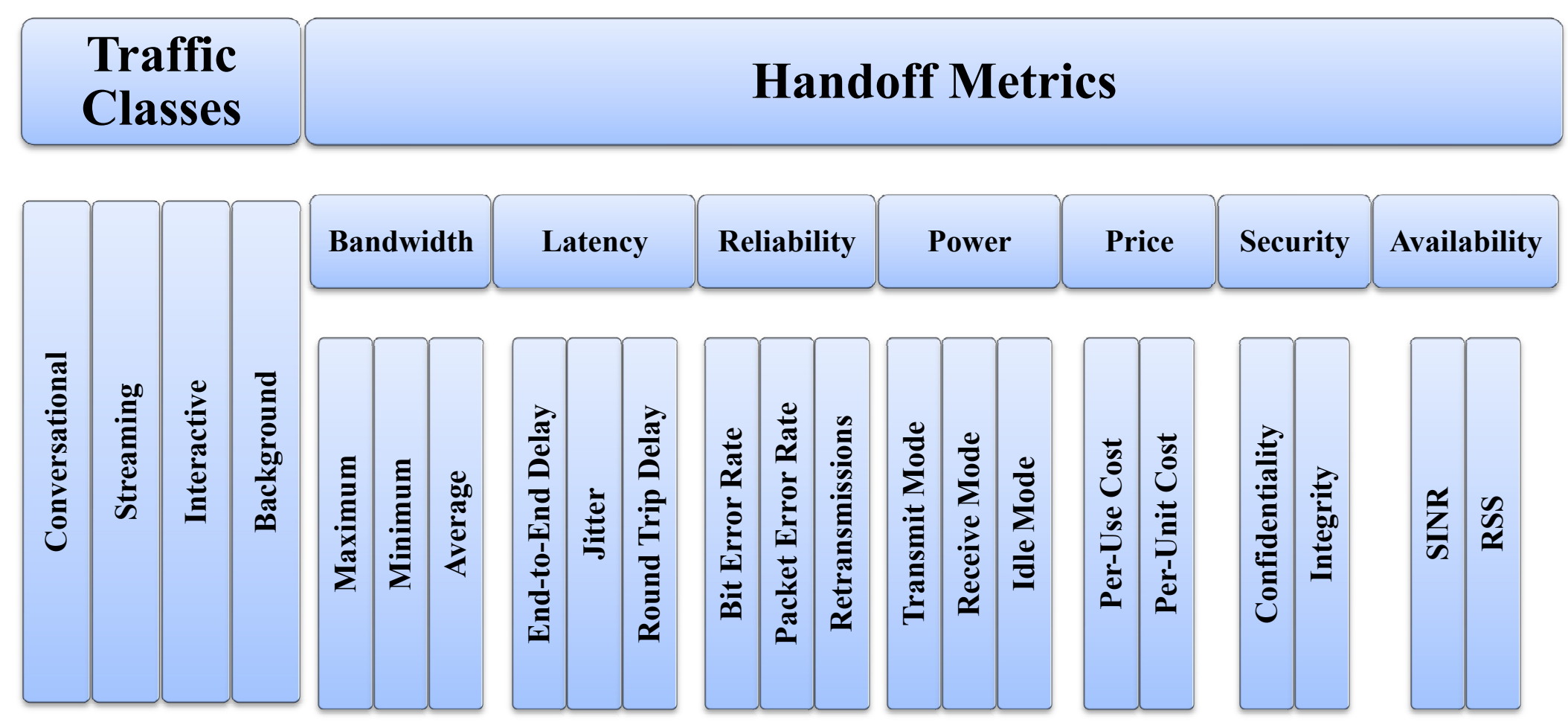

Figure 2.5: Traffic Classes and Handoff Metrics 
- Handoff Delay: This metric represents the time elapsed between the handoff initiation and completion. The complexity of the handoff algorithm has a direct effect on this metric; a simple algorithm results in a smaller value of this metric. A smallest possible value of handoff delay is desired for real-time, delay-sensitive applications.

- Number of Handoffs: Unnecessary handoffs should be minimized as they waste network resources and increase processing overheads.

- Number of Handoff Failures: A handoff failure occurs when the target network fails to allocate sufficient resources for the MS that is handed over from a previous network. This failure is also possible when a moving MS goes out of the coverage area of the target network before the completion of the handoff process. This metric affects the quality of service of an ongoing session.

\subsubsection{IEEE 802.21 FRAMEWORK}

To complete the discussion relating vertical handoffs, it is important to mention the emerging IEEE 802.21 framework. This standard provides a media-independent framework and associated services to enable seamless handoffs between heterogeneous wireless access technologies [34]. The aim of this framework is to improve end-users' satisfaction by enabling seamless handoffs between different wireless technologies, while maintaining session continuity. It is important to note that the standard only provides a framework and that all the implementation details regarding handoff decision-algorithms and executions are left to the design engineers. 


\subsection{LITERATURE REVIEW}

\subsubsection{RSS BASED ALGORITHMS}

In this approach, the RSSs of the different candidate ANs are measured over time and the BS or AP with the strongest signal strength is selected to carry out a handoff. A number of studies $[1,25,35]$ have been conducted in this area due to the simplistic nature of this approach. Since heterogeneous wireless networks comprise of different wireless technologies, their RSSs cannot be compared directly, and thus relative RSS does not apply to vertical handoff decisions. On the other hand, other network parameters such as bandwidth, are typically combined with RSS when making decisions for vertical handoffs. It is important to mention that the possible signal fluctuations due to multipath fading can result in the undesirable so-called "ping-pong effect", i.e., unnecessary handoffs that increases the probability of call failures and drops during the handoff process.

\subsubsection{SIR BASED ALGORITHMS}

Signal to interference ratio is typically used to measure the quality of communication. In this approach, a handoff is initiated if the SIR of the current PoA, BS or AP, is lower than the threshold as compared to the SIR of the target network.

\subsubsection{VELOCITY BASED ALGORITHMS}

Different techniques [36-38] have been presented to perform handoffs, using velocity as the main decision criterion. If the MS in a heterogeneous environment moves with a relatively high velocity, the probability of a call drop may be higher due to excessive delays caused by the handoff process. Based on the velocity of the MS, 
different values of the velocity threshold can be used to make handoff decisions. This is due to the fact that the sojourn time of slower moving MS is much higher than the MS travelling with a relatively higher speed.

\subsubsection{DIRECTION BIASED ALGORITHMS}

For high mobility MSs, this category of algorithms can make effective handoff decisions based on whether the MSs are moving towards or away from the network (BS/AP). This can improve handoff performance by lowering the mean number of handoffs, thus reducing the overall handoff delays. Details of this category of algorithms can be found in $[26,39,40]$.

\subsubsection{MINIMUM POWER ALGORITHMS}

The research work in [41] proposes the use of network's transmission power as a handoff criterion. The proposed technique attempts to find a pair of networks with available channel that has a SIR based on minimum transmitted power. This algorithm reduces call-dropping probability, but increases the number of unnecessary handoffs.

\subsubsection{USER PREFERENCE BASED ALGORITHMS}

These approaches [42-45] mainly take into account the end-users' preferences in terms of MS's power consumption, associated service cost, offered security, and the QoS provided by a candidate network. Most of these approaches are developed to maximize the end-user's satisfaction while utilizing non-real-time applications. 


\subsubsection{CONTEXT AWARE BASED ALGORITHMS}

The approaches presented in [46-52] use context information to perform intelligent handoff decisions. Contextual changes are also taken into account to determine the necessity of handoffs. Context information is collected from the following:

- Mobile Station: Capabilities, remaining battery power, location, and velocity.

- User: User's preferences in terms of preferred network usage-cost, security, and desired QoS.

- Candidate Network: Provided QoS, coverage area, available bandwidth, security offerings, cost of usage, and latency.

- Application: QoS requirements based on the type of service (Conversational, Background, and Streaming, etc.) needed.

\subsubsection{COST FUNCTION BASED ALGORITHMS}

The cost function based approaches [15, 27, 53-59] combine different system's metrics in a cost function that represents a measure of the benefit obtained by handing off to a particular candidate network. For every candidate network, the sum of weighted functions of specific parameters is evaluated to produce the final cost of the network. The general form of a cost function for a wireless network is given by:

$$
f_{n}=\sum_{s} \sum_{i} w_{s, i} \cdot p^{n_{s, i}}
$$

where $p^{n_{s, i}}$ is the cost related to the $i^{t h}$ parameter for providing service $s$ on network $n$, $w_{s, i}$ is the importance weight associated with the $i^{\text {th }}$ parameter and $\sum_{i} w_{i}=1$. 
Two commonly used cost functions found in literature are provided in Equations (2.2) and (2.3).

$$
C_{s}^{n}=\sum w_{s, j}^{n} Q_{s, j}^{n} \quad \text { s.t. } E_{s, j}^{n} \neq 0 \quad \forall s, i
$$

where $C_{s}^{n}$ is the per-service cost for network $n, Q_{s, j}^{n}$ is the normalized QoS provided by network $n$ for parameter $j$ and service $s, w_{s, j}^{n}$ is the weight which indicates the impact of the QoS parameter on the user or the network, and $E_{s, j}^{n}$ is the network elimination factor, indicating whether the minimum requirement of parameter $j$ for service $s$ can be met by network $n$. The second cost function represents the total cost as the sum of all the weighted cost associated with all QoS parameters used.

$$
Q_{i}=w_{c} C_{i}+w_{s} S_{i}+w_{p} P_{i}+w_{d} D_{i}+w_{f} F_{i}
$$

where $Q_{i}$ is the quality factor of network $i ; C_{i}, S_{i}, P_{i}, D_{i}$, and $F_{i}$ are the cost of service, offered security, MS's power consumption, and network conditions \& performance, and $w_{c}, w_{s}, w_{p}, w_{d}$, and $w_{f}$ are the associated weights to the network parameters used. A normalization process

$$
Q_{i}=\frac{w_{c}\left(\frac{1}{C_{i}}\right)}{\max \left(\frac{1}{C_{1}}, \ldots, \frac{1}{C_{n}}\right)}+\frac{w_{S} S_{i}}{\max \left(S_{1}, \ldots, S_{n}\right)}+\frac{w_{p}\left(\frac{1}{P_{i}}\right)}{\max \left(\frac{1}{P_{1}}, \ldots, \frac{1}{P_{n}}\right)}+\frac{w_{d} D_{i}}{\max \left(D_{1}, \ldots, D_{n}\right)}+\frac{w_{f} F_{i}}{\max \left(F_{1}, \ldots, F_{n}\right)}
$$

is used to calculate a normalized quality factor for network $n$. This is required as each network's parameter has a different unit.

\subsubsection{MULTIPLE CRITERIA BASED ALGORITHMS}

This approach is based on a typical MADM problem where the selection of an access network is performed based on multiple attributes measured from all available 
candidate networks. Some of these MADM techniques [21, 60-73] are discussed as follows:

- Simple Additive Weighting (SAW): SAW is the best known and widely used scoring method utilized by $[21,27,61,62,64,70,74]$ to rank candidate networks. A weighted sum of all the network attributes is used to determine the overall score of each candidate network. The score of the $i^{\text {th }}$ candidate network is obtained by adding the normalized contributions from each metric $r_{i j}$ multiplied by the weight $w_{j}$ assigned to the $j^{\text {th }}$ metric. The selected network has the highest score and is given by:

$$
\begin{aligned}
& A_{S A W}=\arg \max _{i} \sum_{j=1}^{N} w_{j} r_{i j} \quad i \in M \\
& r_{i j}=\frac{x_{i j}}{x_{j}^{+}} \quad \text { where } j \in B
\end{aligned}
$$

or

$$
\begin{gathered}
r_{i j}=\frac{x_{j}^{-}}{x_{i j}} \quad \text { where } j \in C \\
x_{j}^{+}=\max _{i \in M} x_{i j} \\
x_{j}^{-}=\min _{i \in M} x_{i j} \\
\sum_{j=1}^{N} w_{j}=1
\end{gathered}
$$

where $x_{i j}$ is the $j^{\text {th }}$ attribute of the $i^{\text {th }}$ network, $N$ is the number of parameters, $M$ denotes the number of candidate networks, $B$ represents benefit type criteria (like throughput), and $C$ represents cost type criteria (like delay). 
- Multiplicative Exponent Weighting (MEW): In these techniques [21, 34, 61], a handoff decision matrix is formed where a particular row and column corresponds to the $i^{\text {th }}$ candidate network and $j^{\text {th }}$ attribute of that network, respectively. The weighted product of the attributes is used to determine the score $S_{i}$ of the $i^{\text {th }}$ network as follows:

$$
S_{i}=\prod_{j=1}^{N} x_{i j}{ }^{w_{j}}
$$

where $x_{i j}$ denotes $j^{t h}$ attribute of the $i^{\text {th }}$ candidate network, $w_{j}$ denotes the weight of attribute $j$, and $\sum_{j=1}^{N} w_{j}=1$. The rank of the selected network is given by:

$$
A_{M E W}=\arg \max _{i} S_{i} \quad i \in M
$$

where $M$ denotes the number of available candidate networks.

- Techniques for Order Preference by Similarity to Ideal Solution: The selected network in the TOPSIS schemes [21, 61, 64-67, 69, 75] is the one that is closest to the ideal solution and the farthest from the worst-case solution. This ideal solution is obtained by using the best value for each metric. The selected network is given by:

$$
A_{\text {TOPSIS }}=-\arg \max _{i} c_{i} \quad i \in M
$$

where $c_{i}$ denotes the relative closeness (similarity) of the candidate network $i$ to the ideal solution. This technique can be applicable to problems spaces for the attributes with monotonically increasing or decreasing levels of utility. The algorithm calculates perceived 
positive/negative ideal solutions based on the range of attribute values available for the alternatives.

- Elimination and Choice Translating Priority (ELECTRE): This is another scheme $[60,61,69,74]$ used to rank the alternatives. The authors in [60] utilize a reference vector of attributes as an ideal alternative to adjust the raw attributes of the candidate networks. A matrix containing the difference between the attribute values of this reference vector and other alternatives is formed, and normalized. The resultant matrix contains attributes that have a monotonically decreasing utility. Weights are assigned to each attribute to take into account their relative importance. Finally, the concept of concordance (measure of satisfaction) and discordance (measure of dissatisfaction) is applied during the comparison of each alternative network with others. A candidate network with the highest value of concordance index and lowest value of discordance index would be the preferred network.

- Analytic Hierarchy Process and Grey Relational Analysis (GRA): The AHP $[67,68,70,72-74,76]$ decomposes the network selection problem into several smaller problems and assigns a weight value to each of them. GRA [77-79] is then used to rank the candidate networks, and the network with the highest rank value is chosen. The Grey Relational Coefficient (GRC) of each network, which describes the similarity between each candidate networks and the ideal network, is calculated. The selected network is given by: 


$$
A_{G R A}=\arg \max _{i} \Gamma_{0, i} \quad i \in M
$$

where $\Gamma_{0, i}$ is the GRC of the $i^{\text {th }}$ network.

In $[72,77]$, the authors propose a combined application of AHP and Grey System theory to evaluate the users' preferences and service requirements, and combine the QoS requirements with the candidate networks' performances to make the final network selection decisions.

- VIKOR: VIKOR $[61,62,80,81]$ is an MADM method that is developed to optimize the multi-attribute based complex systems. It is a compromise programming approach that is based on an aggregating function that represents closeness to the ideal solution. Thus, VIKOR is able to determine a compromise-ranking list of alternatives in the presence of conflicting criteria.

A comparative analysis of some of these methods with numerical examples, for voice and data applications, in a $4 \mathrm{G}$ wireless system is proposed in [21]. It is shown that methods such as SAW, TOPSIS, and VIKOR are suitable for voice connections, whereas GRA and MEW provide a better performance for data connections.

Another comparison of these methods, using bandwidth, delay, jitter, and BER as system's parameters is shown in [61]. Results show that MEW, SAW, and TOPSIS provide similar performance to the four different classes of traffic (Conversational, Streaming, Interactive, and Background) that are used. GRA provides a slightly higher bandwidth and lower delay for Interactive and Background traffic classes. Results also demonstrated that the performance of these algorithms depends on the priority weights assigned to the system parameters. 


\subsubsection{AI BASED APPROACHES}

Just like the cost-function and MADM based approaches, this category of algorithms also takes advantage of combining multiple parameters. In addition, the AI based schemes such as fuzzy logic, neural networks, expert systems, and Genetic Algorithms (GA) are used to perform vertical handoff decisions. As discussed previously, classical techniques, which are based on evaluation of imprecise metrics, fail to perform efficient handoff decisions. Fuzzy logic based schemes can be used to perform efficient handoffs as they can effectively deal with known sensitivities of network parameters with the help of inference rules that are based on expert human knowledge. On the other hand, handoff data can be used to train neural networks to perform these decisions in an efficient manner. Fuzzy logic, combined with ANN or GA, can be used to develop adaptive approaches to make highly optimized vertical handoff decisions. In the following paragraphs, a detailed survey of AI based techniques, to perform these decisions, is presented.

\subsubsection{Fuzzy Logic Based Approaches}

A Fuzzy MADM based numerical solution for vertical handoff decisions is first introduced in [82], where imprecise, or fuzzy data in terms of Linguistic Variables is used to specify network parameters and user preferences in the form of weights. These Linguistic Variables are first converted into crisp numbers using a fuzzy number conversion scale and then classical MADM methods like SAW and TOPSIS are applied to voice and Background traffic. The results indicate that TOPSIS is more sensitive to 
user-preferences and network-attribute values, and that SAW gives relative conservative ranking results.

In [83], the authors demonstrate the use of fuzzy logic together with AHP. Fuzzy Logic is used to calculate the membership values of each parameter measured from different networks while AHP is used to determine the weights associated with these parameters (data rate, usage cost, battery, latency, etc.). These weights are used to evaluate the importance of each network metric based on the network-provider's and the end-users' preferences. The objective of this scheme is to select a wireless network (UMTS, GPRS, and GSM) for a particular service that can satisfy end-users' preferences such as low cost, good RSS, optimum bandwidth, low network latency, high reliability, and long battery life. In a related study [84], AHP is used to trigger the handoff, while fuzzy logic is used to select the best access network among a list of candidate networks that are queued based on the results produced by AHP.

A fuzzy based adaptive handoff management protocol is proposed in [85]. Metrics like MS-velocity and distance are used by the FLS to determine the value of adaptive RSS threshold, which is used to trigger the handoff. The proposed scheme does not consider any QoS related parameters or end-users' preferences.

In [86], the speed of the MS and the loading conditions of the candidate wireless networks (UMTS, WLAN, and WiMAX) are used to determine the best access network. The main objective of this scheme is to improve the handoff efficiency when the MSs are moving at high speeds.

The authors in [87] propose a modular fuzzy-logic based handoff decision algorithm utilizing multiple QoS parameters. The algorithm aims to reduce the 
computational complexity and execution time of a traditional FLS by incorporating multiple parallel fuzzy logic controllers. The scheme divides the handoff parameters into multiple groups, each operating with a different fuzzy engine. The outputs of these separate engines are then fed into another fuzzy engine that determines the degree of overall satisfaction for each of the candidate wireless network. Numerical examples are provided based on multiple WLANs, WiMAXs, and cellular networks and significant reduction in number of rules and execution time, compared against the traditional fuzzy and Adaptive-Network Fuzzy Inference System (ANFIS), has been reported.

In [88], the author creates three separate fuzzifiers based on separate membership functions for three parameters (RSS, velocity, and network-loading) obtained from three different wireless networks ( $3 \mathrm{G}$, WLAN, and WiMAX). The objective of this scheme is to apply fuzzy logic to achieve the normalization of network parameters so that the same parameters measured from different wireless networks can be compared directly by the fuzzy inference engine. The output of the fuzzy inference system is a numerical value that is used to rank each candidate network. This network rank, together with user preferences, is used to determine the best access network. No QoS parameters are taken into consideration in this research work.

A QoS-aware fuzzy-rule-based multi-criteria algorithm is proposed in [89]. A fuzzy logic system that accepts four QoS related parameters as inputs is implemented to calculate a handoff score for all available networks. The network with the highest handoff score is selected as the target of handoff. AHP is used to calculate importance weights for the four different traffic classes. The authors also propose an evaluation model using nonbirth Markov chain with state parameters corresponding to the available networks. The 
primary reason for using a non-birth Markov chain is that an MS can be in a region having no network connectivity or connectivity with more than one network and can go from one state to any other state. The major issue with this scheme is the large number of rules (81 rules from 4 inputs, each with three membership functions) for each of the four different traffic classes. Furthermore, the research does not take into consideration other important parameters like RSS, end-user mobility, and preferences when making handoff decisions.

The scheme developed in [31] is implemented to handle handoffs between UMTS and WLAN. This scheme employs a pre-decision unit to check for two conditions: Condition-1 is to check if the MS is connected to WLAN and if the velocity of the MS is higher than some velocity threshold. In this case, in order to prevent a connection breakdown a handoff to UMTS is directly initiated, disregarding other decision criteria. Condition-2 is checked if the outcome of condition-1 is false. In condition-2, if the predicted RSS (PRSS) from WLAN is greater than its threshold, or if the PRSS measured from UMTS is less than its threshold, no handoff is triggered. After the pre-decision, the fuzzy-logic based Normalized Quantitative Decision (FNQD) is applied. Performance Evaluation Values (PEVs) are generated based on the normalization of current RSS, predicted RSS, and bandwidth. These PEVs are then used to select the target network. The research shows improved performance by reducing the number of unnecessary handoffs and by minimizing the "ping-pong effect". However, calculations of these PEVs are done using fixed weights, which is not practical due to the dynamic wireless network conditions and user requirements. In addition, QoS parameters and end-users' preferences are not considered in this approach. 
A scheme similar to [31] utilizing pre-decision based on MS's motion trends is introduced in [90]. The motion trends of MS and the RSS measured from WLAN are used to pre-select a number of candidate networks. A final comprehensive performance value for each chosen network is then calculated by using fuzzy logic that utilizes RSS, available bandwidth, and the network usage cost. The final handoff decision is based on these performance values and the dwell timer. The dwell timer is incorporated to reduce the "ping-pong effect".

In [91], the authors propose a decision support system to address the vertical handoff problem by combining fuzzy logic and TOPSIS. Several parallel fuzzy logic based subsystems are used to normalize different parameters measured from three different wireless networks. These normalized parameters are then used by TOPSIS to rank the candidate networks. Although the developed scheme shows significant improvements in terms of number of handoffs and handoff failures rate when compared against algorithms where vertical handoff is performed based on only MS velocity, resource availability, or signal strength, the major deficiency of this scheme is the lack of utilization of all QoS related parameters for different traffic classes in order to maximize end-user's satisfaction.

A vertical handoff decision algorithm based on fuzzy logic in conjunction with GPT is presented in [92]. The GPT takes 4 sampled RSSs as input, and predicts the future RSS value to trigger the handoff initiation process. Fuzzy logic theory based Quantitative Decision Algorithm (FQDA) is used to quantitatively evaluate RSS, available bandwidth, and usage cost of the candidate networks. The FQDA produces a Quantitative Decision Value (QDV) for each candidate network that indicates the probability of a certain 
candidate becoming the target of handoff. The final handoff decision is made based on the resulted QDVs. The proposed approach does not take into consideration other factors like offered security, user preferences, and other QoS related parameters.

\subsubsection{Artificial Neural Network Based Approaches}

Although the research work presented in this dissertation does not utilize neural networks, a survey of artificial-neural-network based schemes is provided to complete the discussion related to AI-based handoffs in heterogeneous wireless networks.

In [93], a multi-layer feed-forward neural-network-based AN modeling method, along with an adaptive parameter adjustment algorithm, is presented. The main objective of this scheme is to enhance the end-user's QoS and improve system performance during handoff between WLAN and UMTS. Working mechanism of UMTS and WLAN is modeled using the non-linear relationships between the user-parameters as input and the QoS requirements as output.

The authors in [32] present a vertical handoff manager middleware solution that is based on neural networks to select the best wireless network, based on a set of predefined user-preferences, device's capabilities, and wireless-network features that are carefully selected to provide optimal performance. A decision function based on the weighted factors like cost-of-service, security preference, power consumption, and available bandwidth is used to determine the best network. All four preference parameters, normalized between 0 and 1 in addition to a network classification parameter, are used as inputs to the ANN. A Success rate of $87 \%$ has been reported in finding the 
best wireless network among other available networks. However, other parameters, like load-conditions at the network or MS-velocity, are not considered in this approach.

A Neural Network based handoff algorithm for a joint system of terrestrial and High Altitude Platform Cellular system (HAPS) is presented in [94]. HAPS can provide services to the users staying at the corner of cells or at covered area influenced by shadowing. In this approach a Radial-Basis Function Network (RBFN) is adopted. The inputs to the ANN consist of average-RSS of the current and the target BSs, MS directions estimated by the Multiple Signal Classification (MUSIC) algorithm on an antenna array, and traffic loads of serving and target BSs. Large scale channel propagation like Hata-path-loss model and small scale channel propagation using Shadow-Rayleigh fading is considered while calculating average RSS. The timing advance concept in GSM system and the MS's battery power are used to determine if the MS is currently located in an obstacle area. Performance of the proposed method is compared against the Back Propagation based neural network and the traditional RSS with hysteresis method, using metrics like handoff rate, blocking rate, and call dropping rate. It is reported that the proposed scheme using the RBFN outperforms the other two techniques. However, QoS parameters and user preferences are not given any consideration in this research. Furthermore, the algorithm is only restricted to the cellular networks.

A pattern recognition based algorithm that estimates MS's position and then performs handoff in overlay networks is presented in [29]. The proposed method divides a given path (a road, a passage, or a corridor) in a wireless network into smaller segments called pattern classes that are identified using a unique number. For each segment, signal 
samples of different cells in different networks are combined in a pattern vector that is used to train the Probabilistic Neural Network (PNN). The exact time of handoff is decided based on the estimated pattern class number and thus unnecessary handoffs caused by uncertainty are avoided. The authors reported a 90\% reduction in "ping-ping effect" and a better classification as compared to the traditional algorithms. However, only the straight path is considered, which crossed multiple WLANs and UMTS cells. Furthermore, the algorithm performs handoffs based on only the MS's position and the measured RSS from available networks. No QoS parameters are given any consideration in this study.

\subsubsection{Combination Algorithms}

In [33], the authors use ANFIS to allow "if-then" rules and membership functions to be constructed based on the training data (historical data of the metrics). Two widely used performance metrics, the Received Signal Strength Indicator (RSSI), and Bit Error Rate (BER) are used in the decision process. A reduction in number of rules and increased performance in terms of reduced number of handoff has been reported.

The authors in [95] utilize both neural networks and fuzzy logic to perform vertical handoff decision. The scheme comprises of two steps: first, neural networks are used to predict the value of RSS and then, the time for which an MS stays connected to the network is estimated. These values are used to trigger the handoff. In the second step, FQDA is used to calculate a merit function using RSS, bandwidth, number of users, power consumption, and network usage cost. The merit function in conjunction with a network elimination factor is used to find the target network based on the end-user's 
preferences. The research work demonstrates performance improvements as compared to other traditional approaches. On the other hand, the study doesn't show how quantitative evaluation values (QEVs) utilized as part of the FQDA process, are obtained.

A fuzzy logic and ANFIS based vertical handoff decision-making algorithm [96] is proposed for GSM/GPRS, Wi-Fi, UMTS, and WiMAX networks. The network usage cost, data rate, and the RSSI are used as inputs to the fuzzy logic system to generate an access-point candidacy-value (0-10) that is used to determine the best possible access point. Training data for ANFIS is obtained from the developed fuzzy system and human expert knowledge. Rayleigh-fading is used to emulate multipath propagation channels. The proposed scheme is compared against the developed fuzzy logic and SAW based schemes with number of handoffs being used as the performance evaluation criterion. The simulation is performed for MS moving along a straight path and generating time sensitive voice traffic, GSM based data traffic, and GPRS based data. It is shown in this research that the ANFIS based approach dramatically reduces the number of handoffs and the decision time. An enhanced version of the same research is presented in [97] where the authors replace the ANFIS architecture with GAs to adjust the shape of the fuzzy membership functions. These optimized membership functions are obtained in an offline mode through the application of GAs and later embedded in the developed fuzzy system to make optimal decisions regarding vertical handoffs. Due to the dynamic conditions and inherent uncertainty in the wireless networks, the obtained membership functions may not produce optimal results after a while. Furthermore, QoS parameters and enduser's preferences need to be embedded in the scheme to yield these decisions. 
A multi-criteria ANFIS based vertical handoff decision algorithm is presented in [98] showing throughput gain and reduced "ping-pong effect". Six input parameters including available bandwidth, MS-velocity, number-of-users in WLAN, RSS, MSbattery-level, and the network coverage area are used. Computational complexity is high in the proposed approach. In addition, the research doesn't perform comparisons against any other algorithms. Furthermore, bandwidth is the only QoS parameter that is utilized in this scheme ignoring the other QoS related parameters.

A host mobility support with adaptive network selection method based on neurofuzzy decisions is proposed in [99]. The scheme is based on adaptive fuzzy logic system where the rules are derived and extracted from given training data. An alternative defuzzification approach based on FALCON model [100] is adopted in the proposed scheme. The behavior of a typical user is assumed and response times of services like FTP, web browsing, and VoIP are observed. Traffic model w.r.t different BSs, battery status of MS, and other QoS factors are not considered in this research.

The AI scheme in [101], which is based on a hybrid parallel fuzzy-logic-system, multiple-criteria decision-making system, and GAs, is developed to provide adaptive, flexible, and scalable solution to the vertical handoff decision problem. The decision phase takes advantage of three parallel fuzzy-logic subsystems to reduce the expected complexity involved with the heterogeneous wireless environment by reducing the number of needed inference rules and their complexities. The normalized outputs of these subsystems along with their importance weights that are optimized using GAs, are fed into a multi-criteria decision making system, which utilizes an enhanced version of Simple Multi-Attribute Rate Technique (SMART). Every fuzzy subsystem produces two 
output variables to describe the probability of acceptance for the new MS in the CDMA and TDMA networks. The proposed scheme is compared against three different algorithms using four types of services including voice calls, low bit-rate real-time video telephony, the high bit-rate streaming video, and the non-real-time data traffic. The results show an increase percentage of satisfied users and an overall enhancement of $31 \%$ over the other algorithms. However, the proposed scheme is limited to only four different criteria and does not take into consideration other important decision factors like loading conditions of the network. Furthermore, single-objective GAs are used to optimize each objective weight independently rather than utilizing a multi-objective utilization method to find optimal weights jointly, which could have resulted in an increased performance. The authors extended their work in [102] where a multi-criteria radio network selection solution is presented with co-existed WLAN, WMAN, and WWAN. The scheme utilizes a combined parallel FLCs and an MCDM system to perform handoff decisions. The proposed scheme is based on two modules. The first is a network-controlled mobileassisted module that considers the operator benefits, network conditions and user preferences. The second module is mobile-controlled with network assistance that considering mainly user preferences. Both modules comprise of FLC subsystems that consider one selection criteria and an MCDM. The MCDM is based on an enhanced version of AHP.

A fuzzy multi-criteria vertical handoff algorithm with its parameters enhanced by the use of an inverted 2-layer Multi-Layer Perceptron (MLP) is proposed in [103]. In the proposed approach, a preliminary selection of candidate networks is performed using RSS to reduce the complexity of the fuzzy logic controller. The FLC takes five inputs 
including RSS and loading-conditions of the current and the target systems, and the velocity of MS. A total of 24 fuzzy handoff rules including general rules, UMTS specific rules, and the WLAN specific rules are created. An MLP is used to determine the optimal parameters whenever the system condition changes. The MLP is tested using two backpropagation training algorithm: back-propagation algorithm with an adaptive rate and a momentum, and the resilient back-propagation algorithm. In the proposed approach a 2layer MLP, with FLS parameters as inputs and the desired UMTS and WLAN throughput as outputs, is trained and then inverted using a non-linear system. This inverted MLP that has a lower complexity, satisfactory error rate and converges faster, is used to obtain the most appropriate FLC design parameters for desired UMTS and WLAN throughputs. The proposed approach is compared against an algorithm that is based on fixed coverage and load thresholds. However, wireless networks are highly dynamic in nature resulting in varying load conditions and coverage.

An adaptive multi-criteria vertical handoff system using fuzzy inference system and a modified Elman neural network is proposed in [104]. In this approach, RSS is primarily used to trigger handoff decision process whereas the neural network is used to predict the number of users in the target network. The number of users, along with the MS-velocity, and available bandwidth of the target network are used as inputs to the fuzzy inference system that performs the final decision. The training and the convergence speed of a traditional Elman neural network is too slow, so it is modified by connecting the context nodes to the output nodes with adjustable weights to improve the overall performance. The performance of this proposed scheme is compared against RSS based conventional vertical handoff algorithm and shows that the proposed scheme provides 
better accuracy when performing handoff decisions. However, this research does not provide any comparisons against other neuro-fuzzy based algorithms.

A fuzzy-neural methodology is developed in [105] to perform Joint Radio Resource Management (JRRM) to ensure certain QoS constraints in heterogeneous wireless networks that comprises of WLAN, UMTS, and GSM Enhanced Data for GSM Evolution (EDGE) Radio Access Network (GERAN). The proposed approach is divided into two parts: A JRRM based on fuzzy-neural methodology to select the target network, and a recursive least square predicator capable of estimating in advance JRRM decisions to properly reserve resources for handoff calls. A reinforcement learning procedure is also used in conjunction with FLC to let the algorithm adapts its parameters in order to minimize the desired target value of dissatisfaction probability. This research work is extended in [106] to incorporate additional decision criteria based on users' preferences and operators' policies utilizing AHP techniques. It is shown that the technique outperforms other reference algorithms.

\subsubsection{BRIEF COMPARISON OF THE APPROACHES}

This section provides a comparison of the different handoff approaches that are discussed in the above sections. Since the decision phase is the most important one in vertical handoffs, the network performance, end-user preferences \& satisfactions, efficiency, flexibility, and complexity \& reliability of the overall algorithm must also be considered when evaluating and comparing these handoff approaches.

Based on the discussions in Sections 2.2.1-2.2.10, different combinations of these criteria can be used to perform handoff decisions: Bandwidth, SIR, network-usage-cost, 
user's preferences, QoS preferences, MS's available battery power, delay, throughput, jitter, response time, BER, burst error, packet retransmissions, packet losses, security preferences, network coverage area, RSS, Traffic load, type of provided services, number of active MSs, and speed of MSs. In most cases, a vertical handoff decision strategy involves complex considerations and many tradeoffs must be made to provide an efficient decision mechanism while keeping the overall complexity at the minimum.

In terms of complexity, single criterion based handoff algorithms (especially RSS based) are usually the simplest among all the categories. On the other hand, multi-criteria based handoff algorithms not only require the collection of several network parameters but these parameters must be normalized as well. Hence they are more complex than the RSS based or bandwidth based handoff techniques. AI based handoff techniques are more challenging and complex due to their pre-training and other requirements.

In terms of reliability, AI based handoff algorithms are considered to be the most reliable among all the others, as these systems are trained beforehand. These algorithms are well-suited for vertical handoff decision problem as they can provide accurate solutions by taking into account multiple decision factors.

With respect to overall processing delays, AI based approaches suffer from the highest delay due to increased system complexity. On the other hand, processing delay might be relatively lower in other techniques; nevertheless, these techniques have significantly high handoff failure probabilities, high number of handoffs, and high number of unnecessary handoffs, which reduces the overall system performance. 
Table 2.1: Overall Comparison of Handoff Approaches [14]

\begin{tabular}{|c|c|c|c|c|c|c|}
\hline Handoffs & RSS & $\begin{array}{c}\text { Cost } \\
\text { Function } \\
\text { Features }\end{array}$ & $\begin{array}{c}\text { User } \\
\text { centric } \\
\text { based }\end{array}$ & $\begin{array}{c}\text { MADM } \\
\text { based }\end{array}$ & $\begin{array}{c}\text { Context } \\
\text { Aware } \\
\text { based }\end{array}$ & AI based \\
\hline $\begin{array}{c}\text { Multi- } \\
\text { Criteria }\end{array}$ & No & Yes & Yes & Yes & Yes & Yes \\
\hline $\begin{array}{c}\text { User } \\
\text { Preferences } \\
\text { Consideration }\end{array}$ & No & Low & High & Medium & High & $\begin{array}{c}\text { Medium } \\
\text { to High }\end{array}$ \\
\hline Efficiency & Low & Medium & Medium & High & High & High \\
\hline Flexibility & Low & High & High & High & High & Medium \\
\hline Complexity & Low & Low & Low & Medium & Medium & High \\
\hline $\begin{array}{c}\text { Service } \\
\text { supported }\end{array}$ & $\begin{array}{c}\text { Non- } \\
\text { Real } \\
\text { time }\end{array}$ & Multiple & Multiple & Multiple & Multiple & Multiple \\
types & types & types & types \\
\hline
\end{tabular}

In the context of the above discussion, Table 2.2 [107] provides a comparison of different handoff approaches based on common performance metrics. Table 2.1 [14] provides an overall comparison between different handoff approaches.

As our research work is based on AI techniques, this chapter will be concluded with a summary of their characteristics as follows:

- Relatively Complex than other techniques.

- Typically work with more than one decision criteria.

- More reliable than others.

- Offer handoff accuracy.

- Can avoid ping-pong effect and thus reduce unnecessary handoffs.

- Can reduce corner effect.

- Have the capability to improve handoff latency. 
Table 2.2: The Comparison of Handoff Algorithms [107]

\begin{tabular}{|c|c|c|c|c|c|c|c|c|c|c|}
\hline & \multicolumn{6}{|c|}{ Conventional Handoffs } & \multicolumn{4}{|c|}{ AI based Handoffs } \\
\hline $\begin{array}{c}\begin{array}{c}\text { Handoff } \\
\text { Techniques }\end{array} \\
\text { Features }\end{array}$ & $\begin{array}{c}\text { RSS } \\
\text { based }\end{array}$ & $\begin{array}{l}\text { RSS with } \\
\text { threshold } \\
\text { and/or } \\
\text { Hysteresis }\end{array}$ & $\begin{array}{c}\text { SIR } \\
\text { based }\end{array}$ & $\begin{array}{l}\text { Minimum } \\
\text { Power } \\
\text { Based }\end{array}$ & $\begin{array}{l}\text { Velocity } \\
\text { Adaptive }\end{array}$ & $\begin{array}{c}\text { Direction } \\
\text { Biased }\end{array}$ & $\begin{array}{l}\text { Fuzzy } \\
\text { Logic } \\
\text { based }\end{array}$ & $\begin{array}{l}\text { Neural } \\
\text { Network } \\
\text { based }\end{array}$ & $\begin{array}{c}\text { Prediction } \\
\text { based }\end{array}$ & $\begin{array}{c}\text { Pattern } \\
\text { Recognition } \\
\text { based }\end{array}$ \\
\hline $\begin{array}{c}\text { Resource } \\
\text { Management }\end{array}$ & $\begin{array}{l}\text { Signal } \\
\text { Strength }\end{array}$ & $\begin{array}{l}\text { Signal } \\
\text { Strength }\end{array}$ & $\begin{array}{c}\text { SIR, } \\
\text { Integrated } \\
\text { resource } \\
\text { management }\end{array}$ & $\begin{array}{l}\text { SIR, Power, } \\
\text { Integrated } \\
\text { resource } \\
\text { management }\end{array}$ & $\begin{array}{l}\text { Velocity, } \\
\text { RSS }\end{array}$ & $\begin{array}{l}\text { Direction, } \\
\text { RSS }\end{array}$ & \multicolumn{4}{|c|}{$\begin{array}{c}\text { Multiple decision criteria (RSS, SIR, velocity, User } \\
\text { preferences, QoS parameters, usage cost, available } \\
\text { power, available bandwidth, etc.) }\end{array}$} \\
\hline $\begin{array}{c}\text { Ping-Pong } \\
\text { Effect }\end{array}$ & Yes & Avoided & $\begin{array}{l}\text { Avoided (by } \\
\text { using } \\
\text { hysteresis) }\end{array}$ & NA & Avoided & Avoided & \multicolumn{4}{|c|}{ Avoided } \\
\hline $\begin{array}{l}\text { Handoff } \\
\text { Latency }\end{array}$ & $\begin{array}{c}\text { Relatively } \\
\text { low }\end{array}$ & $\begin{array}{l}\text { Increased } \\
\text { (based on } \\
\text { hysteresis) }\end{array}$ & $\begin{array}{c}\text { Relatively } \\
\text { low }\end{array}$ & NA & Reduced & Reduced & \multicolumn{4}{|c|}{ Reduced } \\
\hline $\begin{array}{c}\text { Number of } \\
\text { Handoffs }\end{array}$ & $\begin{array}{c}\text { Can be } \\
\text { very high }\end{array}$ & Reduced & NA & NA & NA & Low & \multicolumn{4}{|c|}{ Reduced } \\
\hline $\begin{array}{l}\text { Number of } \\
\text { Unnecessary } \\
\text { Handoffs } \\
\end{array}$ & $\begin{array}{c}\text { Can be } \\
\text { very high }\end{array}$ & Reduced & $\begin{array}{l}\text { Relatively } \\
\text { Low }\end{array}$ & $\begin{array}{l}\text { Relatively } \\
\text { High }\end{array}$ & NA & NA & Low & Low & NA & Low \\
\hline $\begin{array}{c}\text { Corner } \\
\text { Effect } \\
\text { (NLOS) }\end{array}$ & Possible & Possible & Possible & Possible & Yes & Yes & \multicolumn{4}{|c|}{ Yes, but can be avoided } \\
\hline
\end{tabular}




\section{CHAPTER 3}

\section{DESIGN OF VHITS}

This chapter introduces the design of VHITS. The proposed scheme has been developed using a combination of different techniques to achieve intelligent and efficient vertical handoffs decisions, while maximizing end-users' satisfaction, in heterogeneous wireless communication networks.

The handoff algorithms based on cost-function combine multiple systems' metrics to choose the target network that offers the highest overall performance. This approach is considered optimal as compared to the other traditional approaches that rely on single system's parameter like RSS or available bandwidth to make handoff decisions [1]. Further enhancement of the said cost-functions, can be done by applying techniques that are based on Artificial Intelligence, Expert Systems, and Machine Learning (for pattern recognition). Hence, an efficient handoff system for heterogeneous wireless networks can be developed using rule-based expert systems utilizing Fuzzy Logic, an Adaptive Neural Fuzzy Inference Expert Systems, or Neural Expert Systems. Due to their inherent parallelism and using the inference rules that can be developed by exploiting the human knowledge of the system, efficient implementation of rule-based expert systems is possible.

To support seamless mobility while an MS roams in a heterogeneous wireless network, vertical handoff necessity estimations and decisions to select a best target network are two important aspects of the overall mobility framework. The handoff initiation and necessity is critically important to keep the unnecessary handoffs and their failures at a low level. On the other hand, to maximize the end-users' satisfaction levels, 
the decision to select the best network among other available candidates also plays an important role.

This chapter begins with a brief comparison of the proposed scheme with other closely matching schemes. The chapter continues with a detailed discussion of the proposed design related to vertical handoff necessity estimation and target network selection modules. MATLAB is used to design these two important modules and their sub-modules.

\subsection{COMPARISON OF VHITS WITH OTHER SCHEMES}

In this section, a candid discussion is provided to highlight the deficiencies in some of the closely related schemes and how VHITS provides an improved design for the overall handoff process.

The scheme in [83] utilizes fuzzy logic with Multiple Objective Decision Making (MODM) approach to select the best network segment. The associated weights of the network parameters are obtained by using the AHP process. This scheme doesn't take advantage of a full Fuzzy Inference System (FIS) and only utilizes the fuzzifier to calculate the membership values of the network parameters. Furthermore, only perceived QoS, without using the QoS related parameters, is considered. In addition, the scheme does not consider WLAN as an alternative and does not provide any solution to the handoff necessity estimation problem. The scheme is evaluated through numerical examples only, without performing any emulation/simulation.

The authors in [84] implement the handoff initiation scheme by combining multiple parameters of all available networks in a cost function. AHP is then used to rank 
all the available networks, including the current PoA. The target network selection is done using a FIS, with velocity and available bandwidth as two inputs, which might not produce optimal results. Furthermore, not all the QoS parameters are taken into consideration while calculating the handoff initiations.

A modular handoff decision system utilizing parallel FLCs is implemented in [87]. The scheme mainly concentrates on QoS related parameters to make handoff decisions. Since, RSS, MS-Velocity, and other important parameters are ignored, the scheme might not provide optimal selection decisions. In addition, no handoff necessity estimation scheme is provided in this work.

The research work in [88] uses parallel FLCs to normalize a subset of important network parameters to rank the network alternatives. No attention is given to the handoff initiation process and the QoS parameters.

A QoS-aware fuzzy-logic based multi-criteria algorithm is proposed in [89]. AHP is utilized to calculate the priority weights of network attributes. Only QoS related parameters are considered to create four FLCs for each of the four different traffic types. The major issue with this scheme is that it relies on bulky rule sets (81 rules) for each FLC, making it inefficient to some extent. Furthermore, the absence of RSS, MSVelocity, and other important parameters may result in non-optimal handoff decisions.

A vertical handoff decision algorithm based on fuzzy logic, in conjunction with GPT, is presented in [92]. Since the scheme uses only the predicted values of RSS to estimate the necessity of handoffs, unnecessary handoffs are likely to occur. Of all available networks, QDVs are produced by utilizing the process of FQDA on RSS, 
available bandwidth, and usage cost. No consideration is given to any other important network parameters including QoS.

A pattern recognition based algorithm that estimates an MS's position to perform handoffs in overlay networks is presented in [29]. To avoid unnecessary handoffs, a PNN is trained to estimate the exact timings of handoff. Although, a reduction in the "pingpong effect" is reported, the scheme does not take into account any QoS related parameters while performing handoff decisions.

By far, the schemes in $[91,101,102]$ are closer to our design where parallel FLCs are used in conjunction with AHP or TOPSIS to produce the final ranking of available networks. The major deficiencies with these schemes are the lack of utilization of all QoS related parameters to perform network selection decisions. Furthermore, these schemes only propose solutions to the network selection problem, while ignoring the handoff necessity estimations.

A fuzzy based MADM scheme is provided in [108] to efficiently deal with uncertainty that is inherent with wireless networks. Parallel FLCs are utilized with two different ranking algorithms: SAW and TOPSIS. However, the scheme pays no attention to the weight elicitation process and arbitrary weights are assigned to conduct simulations for VoIP and Web based traffic classes. Although the scheme considers QoS related parameters, RSS and other important network parameters are ignored while ranking the candidate networks.

The authors in [109] utilize AHP for both weight elicitation and network selection processes. RSS is the only criterion that is used to trigger the handoff. This work is extended in [110] where authors implemented AHP weight elicitation process along with 
TOPSIS to rank the networks. Not all the QoS parameters are utilized to make selection decisions.

A similar scheme for a WiMAX/Wi-Fi environment is provided in [111]. The authors also provided a similar solution in [67] that utilizes AHP and FTOPSIS. However, only numerical examples are provided to evaluate the scheme and no consideration has been given to other important network parameters such as RSS.

A utility-based FTOPSIS method emphasizing only on network power optimization is provided in [112]. TFNs are used to determine the weights for the network parameters. The study only provided numerical examples of network selection for the MS based on all four traffic classes. Some important parameters such as RSS, security, and QoS related parameters are ignored in this work.

A hybrid scheme that utilizes FAHP for weight calculations and ELECTRE to rank the available networks is provided in [113]. Numerical examples, considering only QoS related parameters and cost, are provided.

All the above-mentioned schemes have certain deficiencies and there is no one scheme that provides a complete solution to the vertical handoff problem. Some schemes lack in utilizing important parameters to perform handoff decisions, some do not give any importance to the handoff necessity estimation, some of them are based on bulky fuzzy rule-set, and the others only provide numerical examples to evaluate the scheme without utilizing any simulation test-bed for heterogeneous wireless networks.

The VHITS scheme provides a complete framework to perform vertical handoffs in a heterogeneous wireless network by incorporating the following: 
- A GPT based RSS prediction module is created to minimize handoff failures and call dropping probabilities.

- A handoff necessity estimation module is created that incorporates parallel FLCs with reduced number of rules. This module calculates the handoff necessity based on the predicted RSS, MS-Velocity, weighted QoS parameters based on traffic type, and MS's distance from the current PoA.

- Different weight elicitation techniques are implemented based on both crisp and fuzzy data.

- Several MADM based algorithms including TOPSIS, FTOPSIS, and FVIKOR are utilized to perform network selection. These algorithms utilize a rich set of network parameters to make network selection decisions.

- Simulated scenarios, using a test-bed for heterogeneous wireless environment, are provided in addition to numerical examples to demonstrate the utility of the proposed scheme.

Figure 3.1 shows the flowchart of the overall proposed scheme. In this work, we mainly concentrate on VHITS Necessity Estimation and Target Network Selection mechanisms. The details of these mechanisms are provided in the following sections.

\subsection{VHITS HANDOFF NECESSITY ESTIMATION MODULE}

A multi-attribute vertical handoff decision is more complex than a simple RSSbased horizontal handoff as the former involves attributes from different wireless technologies. In addition, the MS in a heterogeneous wireless environment has the capability to establish and maintain connectivity with many overlay networks that offer 
varying QoSs. Hence, estimating the necessity of a vertical handoff and choosing the right initiation time, reduces the subsequent handoffs, improves the overall QoS, limits the inherent data signaling and rerouting in a vertical handoff process, thus maximizing the end user's satisfaction.

The vertical handoff process should be triggered when any of the following conditions become true $[99,114]$ :

- When the MS detects the availability of a new wireless network or exits the coverage area of the serving network.

- When the MS detects a change in user-preferences. For example, the user decides to switch to a more secure network.

- When the MS detects a request that is made for a new service or if the required QoS for an existing session degrades.

- When there is a severe signal degradation or complete signal loss from the current wireless network.

The proposed VHO necessity estimation module depicted in Figure 3.2 is capable of performing handoffs when most of the conditions, discussed above, become true. In the following, a detail of each block providing support to the VHITS Necessity Estimation module is provided.

\subsubsection{SYSTEM ATTRIBUTES}

There exist various schemes to perform handoffs in heterogeneous wireless networks, based on a combination of different system attributes. Our proposed scheme utilizes a few carefully chosen attributes that are critical to maximizing the end-users' 
satisfaction while performing efficient handoffs. These attributes include network RSS, MS-Velocity, distance between the BS/AP and MS, network loading-conditions, security provided by the network, service cost, and QoS parameters including network throughput, latency, jitter, and Packet Loss Ratio (PLR). Although schemes like [84] also consider the MS's remaining battery status, it is purposely ignored in our proposed scheme as the end-user can control this attribute; for example, by connecting a battery charger while travelling.

The VHITS handoff necessity estimation module takes as inputs the MS-velocity, distance between the BS/AP and the MS, RSS, and QoS of the currently serving network. It is assumed that these values are available to the MS through some mechanism; for example, the GPS module installed in most modern MSs is capable of estimating the MS's velocity. These attributes are monitored and evaluated by our handoff necessity estimation scheme to determine if any of the VHO triggering conditions mentioned above are true. For simplicity, we assume that the MS is equipped with multiple wireless interfaces and it can connect to different types of networks, but at a given instant of time it is connected to only one network type. The types of networks include WLAN, Wireless Metropolitan Area Network (WMAN), and Wireless Wide Area Network (WWAN).

With the exception of distance between the MS and the PoA, these attributes are also utilized in the VHITS Target Network Selection module to determine the best network among a list of candidate networks. 


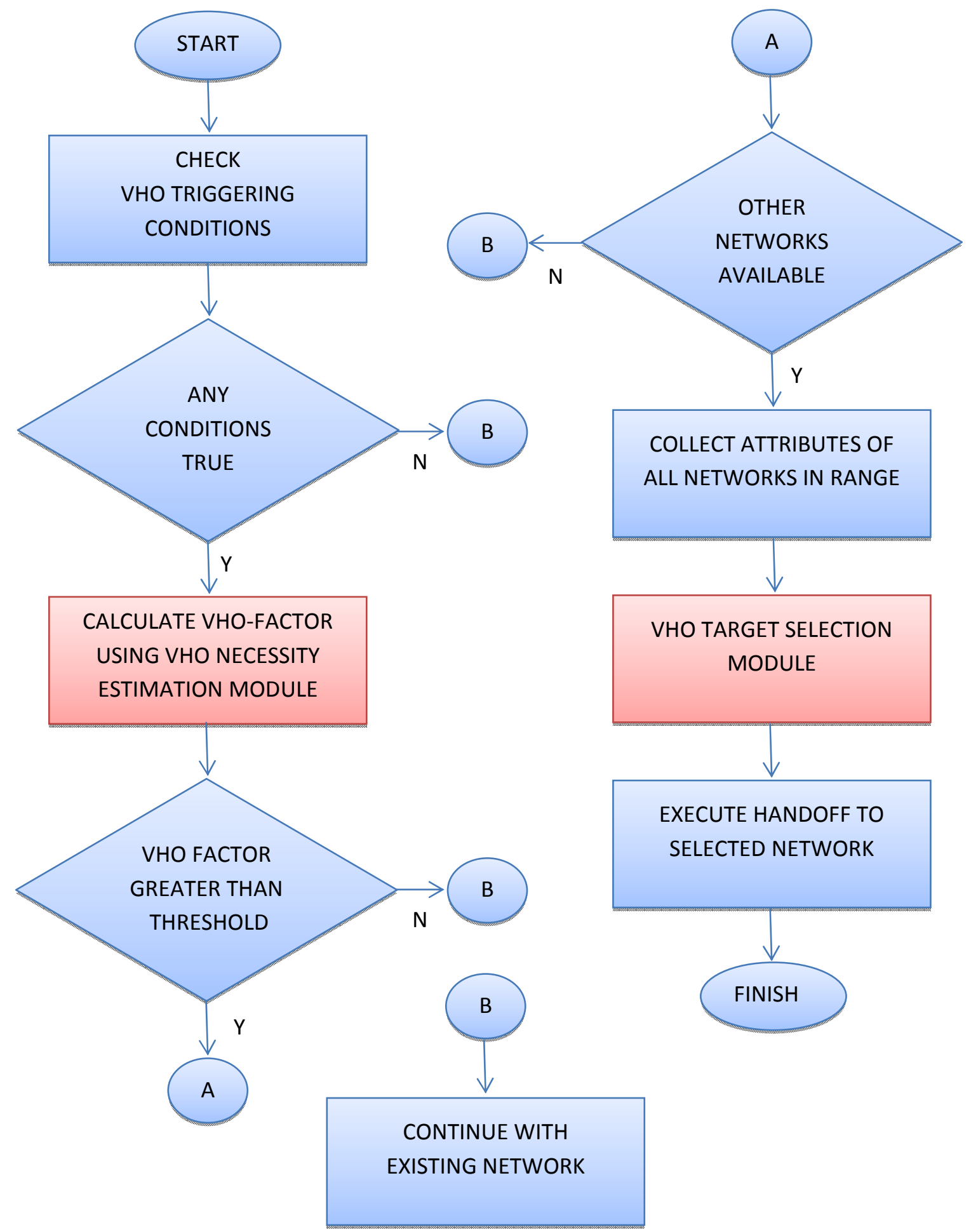

Figure 3.1: VHITS Overall Scheme 


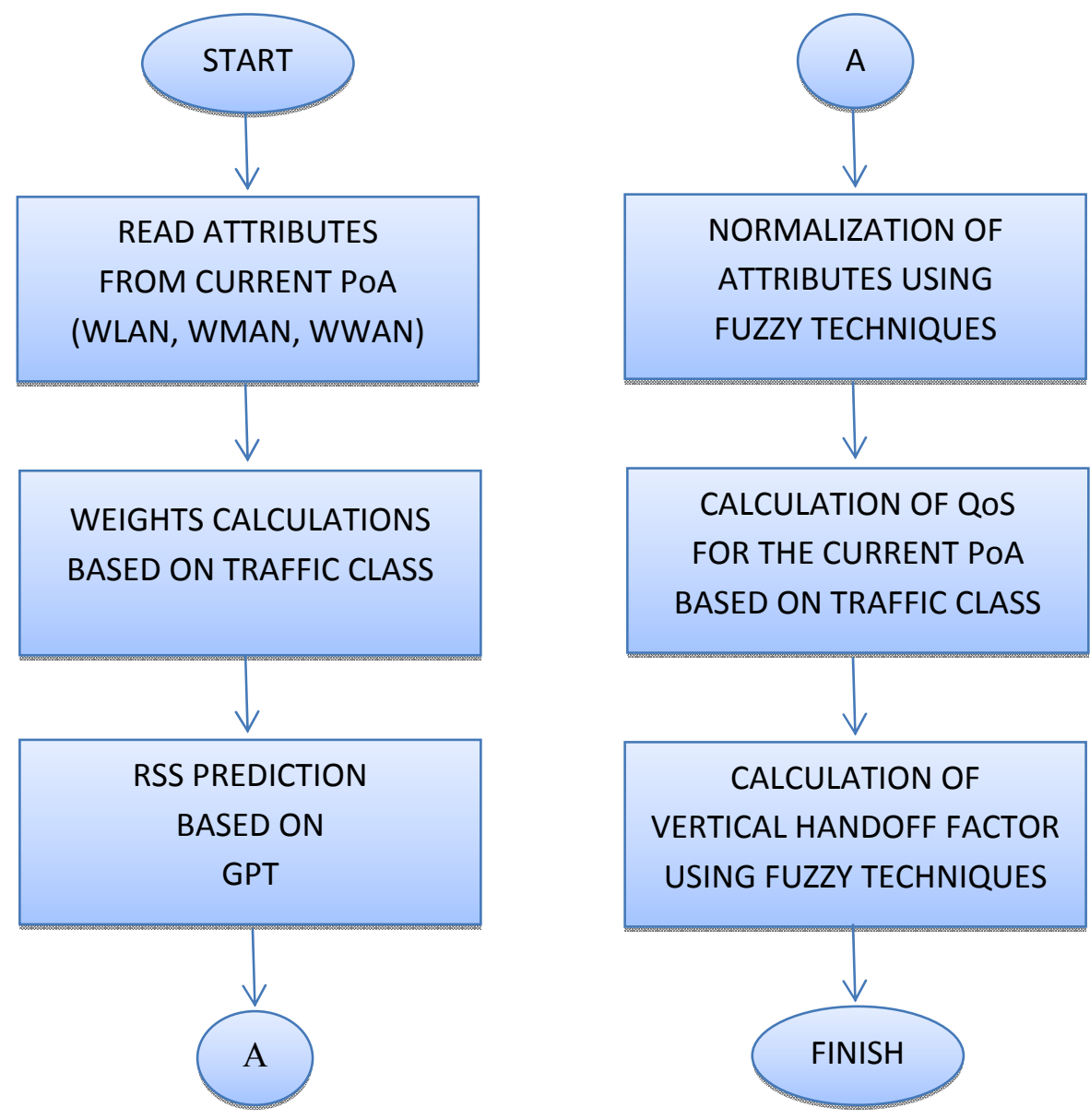

Figure 3.2: VHITS Handoff Necessity Estimation Module

\subsubsection{WEIGHTS CALCULATIONS FOR SYSTEM ATTRIBUTES}

From a decision-making perspective, the end-users can specify their needs and preferences by assigning priority weights to each system attributes. Since the goal of our scheme is to maximize end-users' satisfaction, higher weights are assigned to network RSS and QoS. Furthermore, since QoS requirements vary for various types of traffic classes, different weights with respect to traffic types, need to be calculated and assigned, specifically for QoS related parameters. The proposed scheme considers four different types of traffic classes with different characteristics and QoS demands. These traffic 
classes are defined by 3GPP TS-23.107 specification [115] and are summarized in Table 3.1 .

Note that these priority weights can either be assigned manually to the system attributes or calculated using different techniques as discussed below. Our proposed scheme is flexible and offers both manual weight assignments and calculations using different techniques as will soon be discussed.

\subsubsection{Weights Calculations Using AHP}

The process of making decisions while taking into account more than one criterion is a common task and occurs frequently. Network selection problem exhibits the same characteristic and can be classified as an MADM problem whose goal is to find a network among a set of candidates that can maximize end-users' satisfaction. Different MADM approaches for selecting the best network are presented in Chapter 2. Although, AHP is one commonly used approach to perform the network selection, our scheme only utilizes it to calculate weights for different system's attributes.

The AHP method is introduced by Saaty [76] to find a solution for the complicated problems by dividing such problems into a hierarchy of easy to analyze decision factors and alternatives. AHP performs pairwise comparisons between the attributes, transforms these comparison scores into weights of decision criteria, and prioritizes all alternatives on each criterion to obtain the overall ranking of alternatives. It consists of the following steps: 
Table 3.1: Traffic Classes with varying QoS requirements

\begin{tabular}{|c|c|}
\hline Traffic Classes & Comments \\
\hline Streaming & $\begin{array}{l}\text { - One-way transport } \\
\text { - Example: A user watching a video clip from YouTube or } \\
\text { listening to his favorite radio channel over the Web } \\
\text { - } \text { End-to-end delay is not important }\end{array}$ \\
\hline Interactive & $\begin{array}{l}\text { - Two-way transport that relies on request/response mechanisms } \\
\text { - Example: User chatting with another user using Yahoo } \\
\text { messenger or performing a financial transaction over the Web } \\
\text { - Delay and PLR are important } \\
\text { - Jitter and throughput are relatively less important }\end{array}$ \\
\hline Conversational & $\begin{array}{l}\text { - Two-way transport } \\
\text { - Example: VoIP and video conferencing between end-users } \\
\text { - Delay and Jitter are critically important. } \\
\text { - PLR and throughput are relatively less important }\end{array}$ \\
\hline Background & $\begin{array}{l}\text { - One-way transport } \\
\text { - Example: User sending SMSs or emails } \\
\text { - } \quad \text { PLR is very important } \\
\text { - Delay, Jitter and Throughput are relatively less important }\end{array}$ \\
\hline
\end{tabular}

1. Determination of the objective and the decision factors: In this step, the problem is divided into a hierarchal structure comprised of the main objective, decision attributes, and the available alternatives. At any depth, the decision attributes can be further decomposed into several sub-attributes.

2. Determination of the relative importance of the decision factors: During this phase, pairwise comparisons between the attributes at each level of the 
hierarchy are made. These comparisons are based on how strongly an attribute influences the other attribute in the pair. Table 3.2 depicts a fundamental scale that can be used to perform these comparisons.

For example, while performing a pairwise comparison between two attributes "A1" and "A2", a value of " 5 " is assigned. This simply means that "A1" has a strong influence (5 times) over "A2". On the other hand, a reasonable assumption can be made that "A2" will have a 1/5 influence over "A1".

The comparison results are formulated in a square matrix $A=\left[a_{i j}\right]_{n \times n}$ where $a_{i i}=1, a_{j i}=\frac{1}{a_{i j}}, \quad a_{i j} \neq 0$ and $n$ represents the number of decision attributes.

3. Normalization and Calculation of the relative weights: In this step, relative weights $(w)$ are calculated by normalizing column vector if the matrix is consistent $($ rank $=1)$. In case that the matrix is inconsistent, the largest Eigenvalue/Eigenvector method can be used. First the eigenvalues are calculated by solving $\operatorname{det}(\lambda I-A)=0$, then the normalized weight vector can be obtained as follows:

$$
A w=\lambda_{\max } w
$$

where $A$ is the AHP comparison matrix. A matrix with more than one eigenvalue indicates potential comparative inconsistency within the pairs of attributes. The Consistency Index (CI) and Consistency Ratio (CR) can be used to find these inconsistencies. They are defined as follows:

$$
C I=\frac{\lambda_{\max }-n}{n-1}
$$


Table 3.2: AHP Fundamental Scale of Importance

\begin{tabular}{|c|l|}
\hline Intensity of Importance & \multicolumn{1}{|c|}{ Definition } \\
\hline 1 & Equal Importance \\
\hline 3 & Moderate Importance \\
\hline 5 & Strong Importance \\
\hline 7 & Very Strong Importance \\
\hline 9 & Extreme Importance \\
\hline $2,4,6,8$ & Intermediate Values \\
\hline
\end{tabular}

$$
C R=\frac{C I}{R I}
$$

where $n$ is the number of elements being compared, and $R I$ is the Random Consistency Index that is chosen based on the value of $n$. In practice, $C R \leq 0.1$ is considered acceptable; otherwise the subjective judgment of the decision makers related to the pairwise comparisons needs to be revised.

One of the requirements of AHP is that it assumes independence between any two attributes at the same level of hierarchy. In the network selection problem, where the QoS parameters are also used as decision factors, there exists interdependence between delay, jitter, and PLR. The interdependence between these attributes must be resolved before finalizing the weights of these conflicting attributes. Our proposed scheme resolves this interdependency by repeating the steps of AHP on the conflicting attribute pairs as well.

Figure 3.3 shows the hierarchal structure of all the attributes and sub-attributes utilized by our scheme. The development of this hierarchal structure is the first step towards using AHP. 


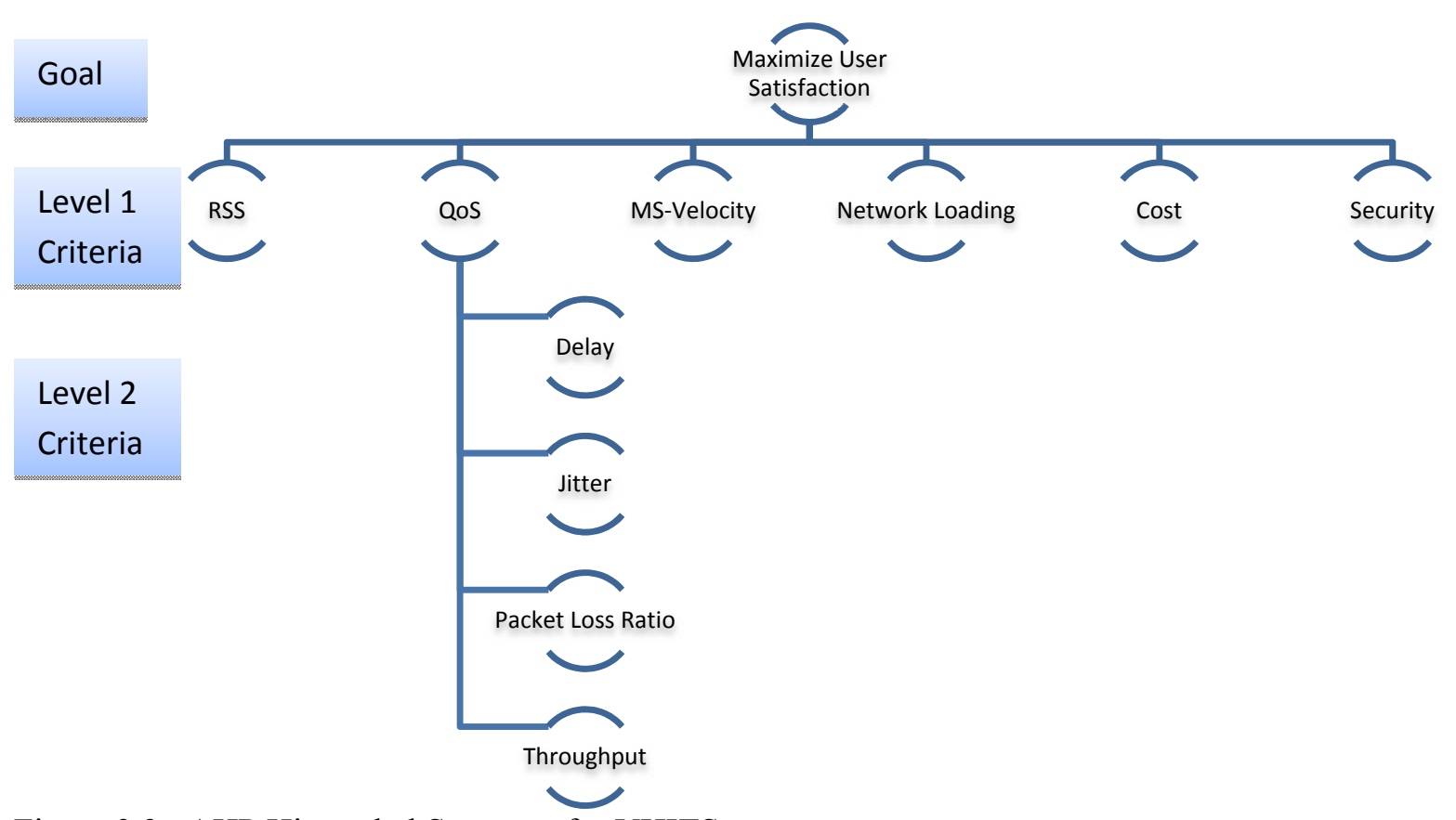

Figure 3.3: AHP Hierarchal Structure for VHITS

The end-user can assign the relative importance of the first-level criteria. On the other hand, the proposed scheme defines the relative importance of the second-level attributes, considering the different requirements related to the QoS for the four traffic classes. The order of preference for level-1 criteria, as utilized in our design, is given as: RSS, QoS, Velocity, Network Loading, Security, and Cost; where RSS and QoS are given equal importance as our goal is to maximize end-users' satisfaction. Nonetheless, our scheme is flexible and the end-users may change this preference order based on their requirements. The detail of the weight calculation process for all four traffic classes is given as follows:

\subsection{Weights for Conversational Traffic Class}

Table 3.3 shows the AHP decision matrix for level-1 criteria. Note that these values are a sample of end-users' subjective assignments of relative importance, and may 
be changed based on their preferences. These values are assigned using the AHP scale of importance given in Table 3.2.

Table 3.4 shows the AHP decision matrix for the relative importance of QoS's sub-criteria based upon the characteristics of Conversational traffic class. As mentioned in Table 3.1, delay and jitter are critical for Conversational traffic class and higher values of these two attributes could result in an unacceptable quality of service. PLR is relatively less important as compared with delay and jitter; humans have the capability of making up the contents of the ongoing conversation, regardless of moderate packet loss. On the other hand, the throughput requirement for Conversational traffic is relatively low and can be supported by all types of networks.

As explained previously, AHP requires that all decision factors, residing at the same level of hierarchy, should not have any interdependence between each other. This is clearly not the case with the QoS parameters (level-2 sub-criteria) as delay, jitter, PLR, and throughput are all related to each other. This interdependence between the QoS parameters can be resolved by repeating the AHP process again by performing various comparisons between these sub-criteria as shown in Tables 3.5-3.7.

The weights obtained in Tables 3.5-3.7 are combined in an interdependence matrix presented in Table 3.8. Note that this interdependence matrix will remain the same for all four traffic classes. The final weights for the four QoS parameters are calculated by multiplying the values in the interdependence matrix with the weights that are obtained in Table 3.4. This is shown in Equation (3.4). 
Table 3.3: AHP Decision Matrix for Level-1 Criteria

\begin{tabular}{|c|c|c|c|c|c|c|c|c|} 
Criteria & RSS & QoS & Velocity & $\begin{array}{r}\text { Network } \\
\text { Loading }\end{array}$ & Security & Cost & Weights & CR \\
\hline RSS & 1 & 1 & 3 & 4 & 5 & 7 & 0.3271 & \\
\hline Q0S & 1 & 1 & 3 & 4 & 5 & 7 & 0.3271 \\
\hline Velocity & $1 / 3$ & $1 / 3$ & 1 & 2 & 3 & 5 & 0.1466 & \multirow{2}{*}{0.0392} \\
\hline Network & $1 / 4$ & $1 / 4$ & $1 / 2$ & 1 & 3 & 5 & 0.1087 & \\
\hline Loading & & & $1 / 3$ & $1 / 3$ & 1 & 3 & 0.0591 & \\
\hline Security & $1 / 5$ & $1 / 5$ & $1 / 3$ & $1 / 5$ & $1 / 3$ & 1 & 0.0314 &
\end{tabular}

Table 3.4: AHP Decision Matrix for QoS Sub-criteria for Conversational Traffic

\begin{tabular}{|c|c|c|c|c|c|c|} 
Criteria & Delay & Jitter & PLR & Throughput & Weights & CR \\
\hline Delay & 1 & 1 & 3 & 7 & 0.3950 & \\
\hline Jitter & 1 & 1 & 3 & 7 & 0.3950 & \multirow{2}{*}{0.0275} \\
\hline PLR & $1 / 3$ & $1 / 3$ & 1 & 5 & 0.1626 & \\
\hline Throughput & $1 / 7$ & $1 / 7$ & $1 / 5$ & 1 & 0.0474 & \\
\hline
\end{tabular}

Table 3.5: AHP Decision Matrix w.r.t Delay

\begin{tabular}{|c|c|c|c|c|}
\hline Criteria & Delay & Jitter & Weights & CR \\
\hline Delay & 1 & 5 & 0.8333 & 0.000 \\
\hline Jitter & $1 / 5$ & 1 & 0.1667 & \\
\hline
\end{tabular}

Table 3.6: AHP Decision Matrix w.r.t Jitter

\begin{tabular}{|c|c|c|c|c|}
\hline Criteria & Jitter & Throughput & Weights & CR \\
\hline Jitter & 1 & 3 & 0.7500 & 0.000 \\
\hline Throughput & $1 / 3$ & 1 & 0.2500 & \\
\hline
\end{tabular}


Table 3.7: AHP Decision Matrix w.r.t PLR

\begin{tabular}{|c|c|c|c|c|c|}
\hline Criteria & Jitter & PLR & Throughput & Weights & CR \\
\hline Jitter & 1 & $1 / 6$ & $1 / 5$ & 0.0811 & \\
\hline PLR & 6 & 1 & 2 & 0.5769 & 0.0279 \\
\hline Throughput & 5 & $1 / 2$ & 1 & 0.3420 & \\
\hline
\end{tabular}

Table 3.8: AHP Interdependence Matrix for QoS parameters

\begin{tabular}{|c|c|c|c|c|}
\hline Criteria & Delay & Jitter & PLR & Throughput \\
\hline Delay & 0.8333 & 0.0000 & 0.0000 & 0.0000 \\
\hline Jitter & 0.1667 & 0.7500 & 0.0811 & 0.0000 \\
\hline PLR & 0.0000 & 0.0000 & 0.5769 & 0.0000 \\
\hline Throughput & 0.0000 & 0.2500 & 0.3420 & 1.0000 \\
\hline
\end{tabular}

$$
W_{\text {QoS-Conv }}=\left[\begin{array}{llll}
0.8333 & 0.0000 & 0.0000 & 0.00 \\
0.1667 & 0.7500 & 0.0811 & 0.00 \\
0.0000 & 0.0000 & 0.5769 & 0.00 \\
0.0000 & 0.2500 & 0.3420 & 1.00
\end{array}\right] \times\left[\begin{array}{l}
0.3950 \\
0.3950 \\
0.1626 \\
0.0474
\end{array}\right]=\left[\begin{array}{l}
0.3292 \\
0.3753 \\
0.0938 \\
0.2017
\end{array}\right] \begin{aligned}
& D \\
& P \\
& T
\end{aligned}
$$

Finally, the overall weightings for all the attributes for the Conversational traffic class are shown in Equation (3.5).

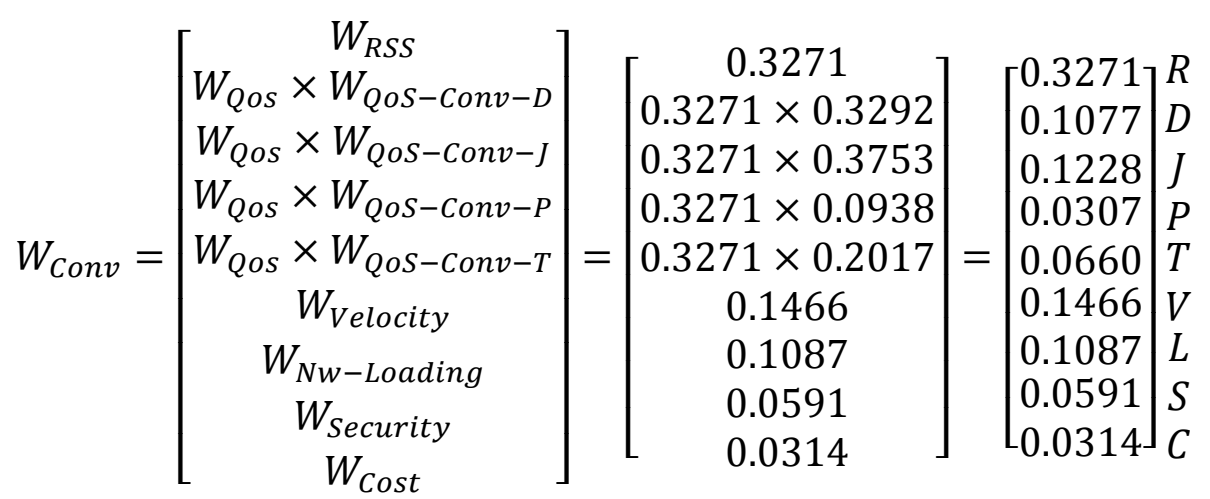


Table 3.9: AHP Decision Matrix for QoS Sub-criteria for Interactive Traffic

\begin{tabular}{|c|c|c|c|c|c|c|} 
Criteria & Delay & Jitter & PLR & Throughput & Weights & CR \\
\cline { 1 - 6 } Delay & 1 & 7 & $1 / 2$ & 3 & 0.3120 & \\
\hline Jitter & $1 / 7$ & 1 & $1 / 8$ & $1 / 3$ & 0.0487 & \multirow{2}{*}{0.0190} \\
\hline PLR & 2 & 8 & 1 & 5 & 0.5230 & \\
\hline Throughput & $1 / 3$ & 3 & $1 / 5$ & 1 & 0.1163 & \\
\hline
\end{tabular}

The final weights for the four QoS parameters for the Interactive class are calculated by multiplying the interdependence matrix (as shown in Table 3.8), with the weights obtained in Table 3.9. This is shown in Equation (3.6).

$$
W_{\text {QoS-Int }}=\left[\begin{array}{llll}
0.8333 & 0.0000 & 0.0000 & 0.00 \\
0.1667 & 0.7500 & 0.0811 & 0.00 \\
0.0000 & 0.0000 & 0.5769 & 0.00 \\
0.0000 & 0.2500 & 0.3420 & 1.00
\end{array}\right] \times\left[\begin{array}{l}
0.3120 \\
0.0487 \\
0.5230 \\
0.1163
\end{array}\right]=\left[\begin{array}{l}
0.2600 \\
0.1310 \\
0.3017 \\
0.3074
\end{array}\right] \begin{gathered}
D \\
P \\
T
\end{gathered}
$$

Finally, the overall weights for all the attributes for the Interactive traffic class are shown in Equation (3.7).

$$
\left.W_{\text {Int }}=\left[\begin{array}{c}
W_{\text {RSS }} \\
W_{\text {Qos }} \times W_{\text {QoS-Int-D }} \\
W_{\text {Qos }} \times W_{\text {QoS-Int-J }} \\
W_{\text {Qos }} \times W_{\text {QoS-Int }-P} \\
W_{\text {Qos }} \times W_{\text {QoS-Int }-T} \\
W_{\text {Velocity }} \\
W_{\text {Nw-Loading }} \\
W_{\text {Security }} \\
W_{\text {Cost }}
\end{array}\right]=\left[\begin{array}{c}
0.3271 \\
0.3271 \times 0.2600 \\
0.3271 \times 0.1310 \\
0.3271 \times 0.3017 \\
0.3271 \times 0.3074 \\
0.1466 \\
0.1087 \\
0.0591 \\
0.0314
\end{array}\right]=\left[\begin{array}{c}
0.3271 \\
0.0850 \\
0.0428 \\
0.0987 \\
0.1005 \\
0.1466 \\
0.1087 \\
0.0591 \\
0.0314
\end{array}\right]_{C}^{R}\right]
$$

\subsection{Weights for Background Traffic Class}

Table 3.10 shows the AHP decision matrix for the relative importance of QoS's sub-criteria based on the characteristics of Background traffic class and the QoS requirements mentioned in Table 3.1. 
Table 3.10: AHP Decision Matrix for QoS Sub-criteria for Background Traffic

\begin{tabular}{|c|c|c|c|c|c|c|}
\hline Criteria & Delay & Jitter & PLR & Throughput & Weights & CR \\
\hline Delay & 1 & 1 & $1 / 5$ & $1 / 9$ & 0.0597 & \\
\hline Jitter & 1 & 1 & $1 / 5$ & $1 / 9$ & 0.0597 & 0.0123 \\
\hline PLR & 9 & 9 & 1 & 3 & 0.6160 & \\
\hline Throughput & 5 & 5 & $1 / 3$ & 1 & 0.2646 \\
\hline
\end{tabular}

The final weights for the four QoS parameters and the overall weightings for all the attributes for the Background traffic class are given in Equations (3.8) and (3.9), respectively.

$$
\begin{aligned}
& W_{\text {QoS-Back }}=\left[\begin{array}{llll}
0.8333 & 0.0000 & 0.0000 & 0.00 \\
0.1667 & 0.7500 & 0.0811 & 0.00 \\
0.0000 & 0.0000 & 0.5769 & 0.00 \\
0.0000 & 0.2500 & 0.3420 & 1.00
\end{array}\right] \times\left[\begin{array}{l}
0.0597 \\
0.0597 \\
0.6160 \\
0.2646
\end{array}\right]=\left[\begin{array}{l}
0.0498 \\
0.1047 \\
0.3554 \\
0.4902
\end{array}\right] \begin{array}{c}
D \\
J \\
T
\end{array} \\
& W_{\text {Back }}=\left[\begin{array}{c}
W_{\text {RSS }} \\
W_{\text {Qos }} \times W_{\text {QoS-Back-D }} \\
W_{\text {Qos }} \times W_{\text {QoS-Back-J }} \\
W_{\text {QoS }} \times W_{\text {QoS-Back-P }} \\
W_{\text {Qos }} \times W_{\text {QoS-Back-T }} \\
W_{\text {Velocity }} \\
W_{\text {Nw-Loading }} \\
W_{\text {Security }} \\
W_{\text {Cost }}
\end{array}\right]=\left[\begin{array}{c}
0.3271 \\
0.3271 \times 0.0498 \\
0.3271 \times 0.1047 \\
0.3271 \times 0.3554 \\
0.3271 \times 0.4902 \\
0.1466 \\
0.1087 \\
0.0591 \\
0.0314
\end{array}\right]=\left[\begin{array}{c}
0.3271 \\
0.0163 \\
0.0342 \\
0.1163 \\
0.1604 \\
0.1466 \\
0.1087 \\
0.0591 \\
0.0314
\end{array}\right] \text { L }
\end{aligned}
$$

\subsection{Weights for Streaming Traffic Class}

The AHP decision matrix, for the relative importance of QoS's sub-criteria based upon the characteristics of Streaming traffic class, is shown in Table 3.11. These pairwise comparisons are based on the characteristic and QoS requirements for Streaming traffic class, as provided in Table 3.1. 
Table 3.11: AHP Decision Matrix for QoS Sub-criteria for Streaming Traffic

\begin{tabular}{|c|c|c|c|c|c|c|} 
Criteria & Delay & Jitter & PLR & Throughput & Weights & CR \\
\hline Delay & 1 & $1 / 5$ & $1 / 6$ & $1 / 7$ & 0.0501 & \\
\hline Jitter & 5 & 1 & $1 / 2$ & $1 / 2.5$ & 0.1902 & \multirow{2}{*}{0.0262} \\
\hline PLR & 6 & 2 & 1 & $1 / 2$ & 0.2971 & \\
\hline Throughput & 7 & 2.5 & 2 & 1 & 0.4626 & \\
\hline
\end{tabular}

Equations (3.10) and (3.11) show the final QoS parameters weights and the overall weightings for all the attributes for the Streaming traffic class.

$$
\begin{aligned}
& W_{\text {QoS-Str }}=\left[\begin{array}{llll}
0.8333 & 0.0000 & 0.0000 & 0.00 \\
0.1667 & 0.7500 & 0.0811 & 0.00 \\
0.0000 & 0.0000 & 0.5769 & 0.00 \\
0.0000 & 0.2500 & 0.3420 & 1.00
\end{array}\right] \times\left[\begin{array}{l}
0.0501 \\
0.1902 \\
0.2971 \\
0.4626
\end{array}\right]=\left[\begin{array}{l}
0.0417 \\
0.1751 \\
0.1714 \\
0.6118
\end{array}\right] \begin{array}{c}
D \\
P \\
T
\end{array}
\end{aligned}
$$

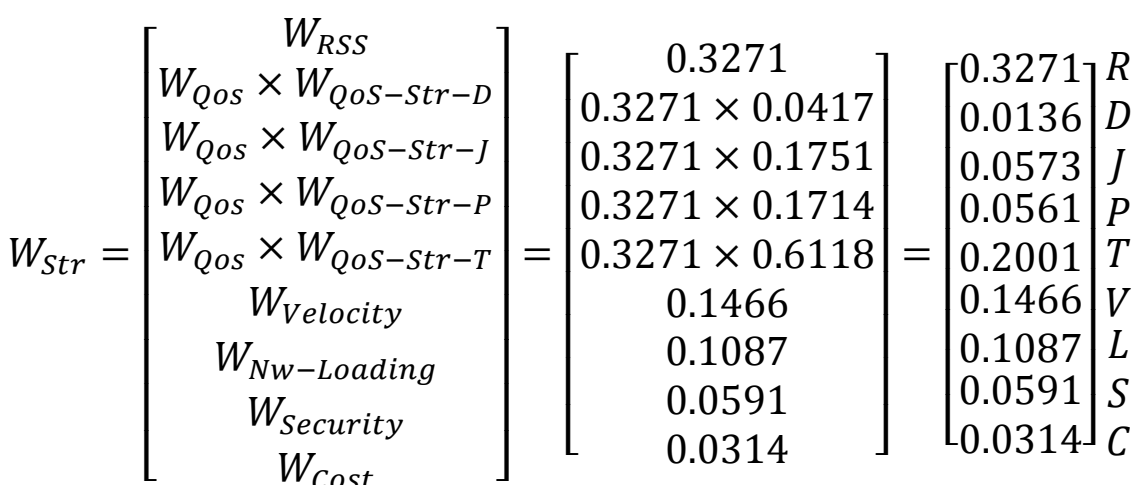

\subsubsection{Weights Calculations Using FAHP}

Despite its popularity, the conventional AHP methodology is often criticized for its failure to effectively handle the intrinsic imprecision and fuzziness associated with the mapping of the end-user's preferences to crisp numbers. Fuzzy Logic can be utilized to deal with this uncertainty by expressing the pairwise comparisons of the decision factors, 
at the same level of hierarchy, as fuzzy sets or fuzzy numbers. To solve hierarchal fuzzy problems, FAHP [116], a fuzzy extension of AHP, has been developed.

In FAHP, the nine judgment levels that are used as the fundamental scale in the original AHP algorithm are expressed via the Triangular Fuzzy Numbers (TFN) to represent the relative importance among the pair of decision factors. A TFN, expressed as $\tilde{x}=(l, m, u)$, is a special type of fuzzy number whose membership value is defined by three real numbers, where parameter $m$ is the most promising value as it gives the maximal grade of the membership function $\mu(x)$, and parameters $l$ and $u$ are the lower and upper bounds that limit the field of the possible evaluation [117]. The membership function of a TFN is given in Equation (3.12).

$$
\mu(x)= \begin{cases}\frac{(x-l)}{(m-l)} & x \in[l, m] \\ \frac{(u-x)}{(u-m)} & x \in[m, u] \\ 0 & \text { otherwise }\end{cases}
$$

Following, are the steps involved in classical FAHP process:

1. In the first step, AHP matrices, containing pairwise comparisons of attributes, are obtained from multiple decision makers.

$$
A^{p}=\left[a_{i j}\right]_{n \times n}^{p}=\left[\begin{array}{cccc}
a_{11}^{p} & a_{12}^{p} & \cdots & a_{1 n}^{p} \\
a_{21}^{p} & a_{22}^{p} & \cdots & a_{2 n}^{p} \\
\vdots & \vdots & & \vdots \\
a_{n 1}^{p} & a_{n 2}^{p} & & a_{n n}^{p}
\end{array}\right] \quad p=1,2, \ldots, t
$$

where $p$ represents the number of decision makers, and $i=j=1,2, \ldots, n$.

2. TFNs representing grades of multiple decision makers are obtained via Equation (3.13). 


$$
\begin{aligned}
& l_{i j}=\min _{p}\left(a_{i j}^{p}\right), \quad m_{i j}=\frac{\sum_{p=1}^{t} a_{i j}^{p}}{p}, \quad u_{i j}=\max _{p}\left(a_{i j}^{p}\right) \\
& \text { where } p=1,2, \ldots, t \text { and } i=j=1,2, \ldots, n
\end{aligned}
$$

3. Establish the FAHP comparison matrix that contains TFNs representing pairwise comparisons between the attributes at a certain level of hierarchy. One such matrix is given in Equation (3.14).

$$
\begin{aligned}
& \tilde{A}=\left(\tilde{a}_{i j}\right)_{n \times n}=\left[\begin{array}{cccc}
(1,1,1) & \left(l_{12}, m_{12}, u_{12}\right) & \cdots & \left(l_{1 n}, m_{1 n}, u_{1 n}\right) \\
\left(l_{21}, m_{21}, u_{21}\right) & (1,1,1) & \cdots & \left(l_{2 n}, m_{2 n}, u_{2 n}\right) \\
\vdots & \vdots & \vdots \\
\left(l_{n 1}, m_{n 1}, u_{n 1}\right) & \left(l_{n 2}, m_{n 2}, u_{n 2}\right) & (1,1,1)
\end{array}\right] \\
& \text { where }\left(\tilde{a}_{j i}\right)=\left[\tilde{a}_{i j}\right]^{-1}=\left(l_{i j}, m_{i j}, u_{i j}\right)^{-1}=\left(\frac{1}{u_{i j}}, \frac{1}{m_{i j}}, \frac{1}{l_{i j}}\right)
\end{aligned}
$$

4. Weights of the attributes are acquired using Fuzzy Extent Analysis [118]. Equation (3.15) is used to obtain the value of the fuzzy synthetic extent with respect to the $i^{\text {th }}$ object.

$$
\tilde{S}_{i}=\sum_{j=1}^{n} \tilde{a}_{i j} \times\left[\sum_{i=1}^{n} \sum_{j=1}^{n} \tilde{a}_{i j}\right]^{-1}
$$

$$
\text { where } \quad \sum_{j=1}^{n} \tilde{a}_{i j}=\left(\sum_{j=1}^{n} l_{i j}, \sum_{j=1}^{n} m_{i j}, \sum_{j=1}^{n} u_{i j}\right)
$$

and $\quad\left[\sum_{i=1}^{n} \sum_{j=1}^{n} \tilde{a}_{i j}\right]^{-1}=\left(\frac{1}{\sum_{i=1}^{n} \Sigma_{j=1}^{n} u_{i j}}, \frac{1}{\sum_{i=1}^{n} \sum_{j=1}^{n} m_{i j}}, \frac{1}{\sum_{i=1}^{n} \sum_{j=1}^{n} l_{i j}}\right)$

5. Calculate the degree of possibility for a convex fuzzy number to be greater than $k$ convex fuzzy numbers $\tilde{S}_{i}(i=1,2, \ldots, k)$. This is given below:

$$
\begin{gathered}
V\left(\tilde{S} \geq \tilde{S}_{i}\right)=V\left[\left(\tilde{S} \geq \tilde{S}_{1}\right) \text { and }\left(\tilde{S} \geq \tilde{S}_{2}\right) \text { and } \ldots \text { and }\left(\tilde{S} \geq \tilde{S}_{k}\right)\right] \\
V\left(\tilde{S} \geq \tilde{S}_{i}\right)=\min \left\{V\left(\tilde{S} \geq \tilde{S}_{i}\right)\right\}
\end{gathered}
$$

where the degree of possibility of $\tilde{S}_{1} \geq \tilde{S}_{2}$ and $\tilde{S}_{2} \geq \tilde{S}_{1}$ is given as, 


$$
\begin{gathered}
V\left(\tilde{S}_{1} \geq \tilde{S}_{2}\right)=\left\{\begin{array}{cc}
1 & m_{1} \geq m_{2} \\
0 & l_{2} \geq u_{1} \\
\frac{l_{2}-u_{1}}{\left(m_{1}-u_{1}\right)-\left(m_{2}-l_{2}\right)}, & \text { otherwise }
\end{array}\right\} \\
V\left(\tilde{S}_{2} \geq \tilde{S}_{1}\right)=\left\{\begin{array}{cc}
1 & m_{2} \geq m_{1} \\
0 & l_{1} \geq u_{2} \\
\frac{l_{1}-u_{2}}{\left(m_{2}-u_{2}\right)-\left(m_{1}-l_{1}\right)}, & \text { otherwise }
\end{array}\right\}
\end{gathered}
$$

Note that in order to compare $\tilde{S}_{1}$ and $\tilde{S}_{2}$, both values of $V\left(\tilde{S}_{1} \geq \tilde{S}_{2}\right)$ and $V\left(\tilde{S}_{2} \geq \tilde{S}_{1}\right)$ are required.

6. Assuming that $d_{i}^{\prime}=\min \left\{V\left(\tilde{S}_{i} \geq \tilde{S}_{k}\right)\right\}$, the weight vector is given by:

$$
w^{\prime}=\left(d_{1}^{\prime}, d_{2}^{\prime}, \ldots d_{n}^{\prime}\right)^{T}
$$

7. Finally, the normalized non-fuzzy weight vector is given in Equation (3.20).

$$
W=\left(d_{1}, d_{2}, \ldots, d_{n}\right)^{T}=\left(\frac{d_{1}^{\prime}}{\sum_{i=1}^{n} d_{i}^{\prime}}, \frac{d_{2}^{\prime}}{\sum_{i=1}^{n} d_{i}^{\prime}}, \ldots, \frac{d_{n}^{\prime}}{\sum_{i=1}^{n} d_{i}^{\prime}}\right)
$$

In the proposed scheme, the FAHP algorithm is coded using MATLAB to calculate the weights for attributes based on different traffic classes. The developed algorithm is capable of taking one or more decision matrices. For the case when only a single decision matrix is available, pre-defined TFNs are used to define the end-user's preferences. These TFNs and their corresponding reciprocal values are defined in Table 3.12 .

In this newly developed algorithm, similar steps as discussed in Section 3.2.2.1 are followed to calculate the weights for each attribute, with the exception of applying FAHP instead of classical AHP algorithm. In the following paragraphs, we will summarize the calculated weights for all of the four traffic classes using FAHP approach. 
Table 3.12: TFNs and Reciprocal TFNs for FAHP Levels of Importance

\begin{tabular}{|c|l|c|c|} 
Intensity of & \multicolumn{1}{|c|}{ Definition } & TFN & $\begin{array}{c}\text { Reciprocal } \\
\text { TFN }\end{array}$ \\
\hline 1 & Equal Importance & $(1,1,1)$ & $(1,1,1)$ \\
\hline 2 & Intermediate Values & $(1 / 2,3 / 4,1)$ & $(1,4 / 3,2)$ \\
\hline 3 & Moderate Importance & $(2 / 3,1,3 / 2)$ & $(2 / 3,1,3 / 2)$ \\
\hline 4 & Intermediate Values & $(1,3 / 2,2)$ & $(1 / 2,2 / 3,1)$ \\
\hline 5 & Strong Importance & $(3 / 2,2,5 / 2)$ & $(2 / 5,1 / 2,2 / 3)$ \\
\hline 6 & Intermediate Values & $(2,5 / 2,3)$ & $(1 / 3,2 / 5,1 / 2)$ \\
\hline 7 & Very Strong Importance & $(5 / 2,3,7 / 2)$ & $(2 / 7,1 / 3,2 / 5)$ \\
\hline 8 & Intermediate Values & $(3,7 / 2,4)$ & $(1 / 4,2 / 7,1 / 3)$ \\
\hline 9 & Extreme Importance & $(7 / 2,4,9 / 2)$ & $(2 / 9,1 / 4,2 / 7)$ \\
\hline
\end{tabular}

\subsection{Weights for Conversational Traffic Class}

Tables 3.13-3.14 show FAHP decision matrices for level-1 and level-2 criteria for the Conversational traffic class, respectively. The tables also display the corresponding TFNs and the weights for all attributes based on the FAHP algorithm. The TFNs generated in Tables 3.15-3.17 are used to create the interdependence matrix of QoS parameters given in Table 3.18.

Finally, the overall weightings for all the attributes for the Conversational traffic class are shown in Equation (3.21).

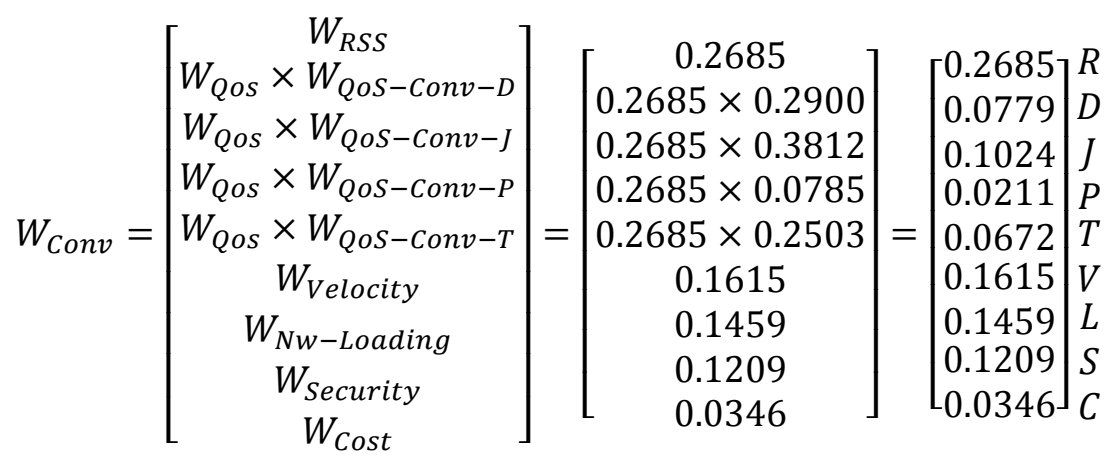


Table 3.13: FAHP Decision Matrix for Leve-1 Criteria for Conversational Traffic

\begin{tabular}{|c|c|c|c|c|c|c|c|}
\hline Criteria & RSS & QoS & Velocity & $\begin{array}{l}\text { Network } \\
\text { Loading }\end{array}$ & Security & Cost & Weights \\
\hline RSS & $\begin{array}{c}1 \\
(1,1,1)\end{array}$ & $\begin{array}{c}1 \\
(1,1,1)\end{array}$ & $\begin{array}{c}3 \\
(0.667,1,1.5)\end{array}$ & $\begin{array}{c}4 \\
(1,1.5,2)\end{array}$ & $\begin{array}{c}5 \\
(1.5,2,2.5)\end{array}$ & $\begin{array}{c}7 \\
(2.5,3,3.5)\end{array}$ & 0.2685 \\
\hline QoS & $\begin{array}{c}1 \\
(1,1,1)\end{array}$ & $\begin{array}{c}1 \\
(1,1,1)\end{array}$ & $\begin{array}{c}3 \\
(0.667,1,1.5)\end{array}$ & $\begin{array}{c}4 \\
(1,1.5,2)\end{array}$ & $\begin{array}{c}5 \\
(1.5,2,2.5)\end{array}$ & $\begin{array}{c}7 \\
(2.5,3,3.5)\end{array}$ & 0.2685 \\
\hline Velocity & $\begin{array}{c}1 / 3 \\
(0.667,1,1.5)\end{array}$ & $\begin{array}{c}1 / 3 \\
(0.667,1,1.5)\end{array}$ & $\begin{array}{c}1 \\
(1,1,1)\end{array}$ & $\begin{array}{c}2 \\
(0.5,0.75,1)\end{array}$ & $\begin{array}{c}3 \\
(0.667,1,1.5)\end{array}$ & $\begin{array}{c}4 \\
(1,1.5,2)\end{array}$ & 0.1615 \\
\hline $\begin{array}{l}\text { Network } \\
\text { Loading }\end{array}$ & $\begin{array}{c}1 / 4 \\
(0.5,0.667,1)\end{array}$ & $\begin{array}{c}1 / 4 \\
(0.5,0.667,1)\end{array}$ & $\begin{array}{c}1 / 2 \\
(1,1.33,2)\end{array}$ & $\begin{array}{c}1 \\
(1,1,1)\end{array}$ & $\begin{array}{c}2 \\
(0.5,0.75,1)\end{array}$ & $\begin{array}{c}4 \\
(1,1.5,2)\end{array}$ & 0.1459 \\
\hline Security & $\begin{array}{c}1 / 5 \\
(0.4,0.5,0.667)\end{array}$ & $\begin{array}{c}1 / 5 \\
(0.4,0.5,0.667)\end{array}$ & $\begin{array}{c}1 / 3 \\
(0.667,1,1.5)\end{array}$ & $\begin{array}{c}1 / 2 \\
(1,1.33,2)\end{array}$ & $\begin{array}{c}1 \\
(1,1,1)\end{array}$ & $\begin{array}{c}3 \\
(0.667,1,1.5)\end{array}$ & 0.1209 \\
\hline Cost & $\begin{array}{c}1 / 7 \\
(0.286,0.33,0.4)\end{array}$ & $\begin{array}{c}1 / 7 \\
(0.286,0.33,0.4)\end{array}$ & $\begin{array}{c}1 / 4 \\
(0.5,0.667,1)\end{array}$ & $\begin{array}{c}1 / 4 \\
(0.5,0.667,1)\end{array}$ & $\begin{array}{c}1 / 3 \\
(0.667,1,1.5)\end{array}$ & $\begin{array}{c}1 \\
(1,1,1)\end{array}$ & 0.0346 \\
\hline
\end{tabular}

Table 3.14: FAHP Decision Matrix for QoS Sub-criteria for Conversational Traffic

\begin{tabular}{|r|c|c|c|c|c|}
\hline Criteria & Delay & Jitter & PLR & Throughput & Weights \\
\hline Delay & 1 & 1 & 3 & 7 & 0.4238 \\
\hline Jitter & $(1,1,1)$ & $(1,1,1)$ & $(0.667,1,1.5)$ & $(2.5,3,3.5)$ & \\
\hline PLR & 1 & 1 & 3 & 5 & 0.3207 \\
\hline & $(1,1,1)$ & $(1,1,1)$ & $(0.667,1,1.5)$ & $(1.5,2,2.5)$ & \\
\hline Throughput & $1 / 3$ & $1 / 3$ & 1 & 3 & 0.2356 \\
& $(0.667,1,1.5)$ & $(0.667,1,1.5)$ & $(1,1,1)$ & $(0.667,1,1.5)$ & \\
\hline & $1 / 7$ & $1 / 5$ & $1 / 3$ & 1 & 0.0199 \\
\hline
\end{tabular}

Table 3.15: FAHP Decision Matrix w.r.t Delay

\begin{tabular}{|c|c|c|c|}
\hline Criteria & Delay & Jitter & Weights \\
\hline Delay & 1 & 4 & 0.6842 \\
& $(1,1,1)$ & $(1,1.5,2)$ & \\
\hline Jitter & $1 / 4$ & 1 & 0.3158 \\
& $(0.5,0.667,1)$ & $(1,1,1)$ & \\
\hline
\end{tabular}


Table 3.16: FAHP Decision Matrix w.r.t Jitter

\begin{tabular}{c|c|c|c|} 
Criteria & Jitter & Throughput & Weights \\
\hline Jitter & $\begin{array}{c}1 \\
(1,1,1)\end{array}$ & $\begin{array}{c}(0.667,1,1.5) \\
\end{array}$ & 0.5000 \\
\hline Throughput & $\begin{array}{c}1 / 3 \\
(0.667,1,1.5)\end{array}$ & $\begin{array}{c}1 \\
(1,1,1)\end{array}$ & 0.5000 \\
\hline
\end{tabular}

Table 3.17: FAHP Decision Matrix w.r.t PLR

\begin{tabular}{|c|c|c|c|c|}
\hline Criteria & Jitter & PLR & Throughput & Weights \\
\hline Jitter & 1 & $1 / 3$ & $1 / 2$ & 0.3694 \\
& $(1,1,1)$ & $(0.667,1,1.5)$ & $(1,1.33,2)$ & \\
\hline PLR & 3 & 1 & 3 & 0.3330 \\
\hline Throughput & $(0.667,1,1.5)$ & $(1,1,1)$ & $(0.667,1,1.5)$ & \\
\hline & $(0.5,0.75,1)$ & $(0.667,1,1.5)$ & $(1,1,1)$ & 0.2976 \\
\hline
\end{tabular}

Table 3.18: FAHP Interdependence Matrix for QoS parameters

\begin{tabular}{|c|c|c|c|c|}
\hline Criteria & Delay & Jitter & PLR & Throughput \\
\hline Delay & 0.6842 & 0.0000 & 0.0000 & 0.0000 \\
\hline Jitter & 0.3158 & 0.5000 & 0.3694 & 0.0000 \\
\hline PLR & 0.0000 & 0.0000 & 0.3330 & 0.0000 \\
\hline Throughput & 0.0000 & 0.5000 & 0.2976 & 1.0000 \\
\hline
\end{tabular}

\subsection{Weights for Interactive Traffic Class}

Table 3.19 shows FAHP decision matrix for level-2 criteria for the Interactive traffic class using TFNs and FAHP algorithm. The overall weights for all the attributes for the Interactive traffic class are shown in Equation (3.22). 
Table 3.19: FAHP Decision Matrix for QoS Sub-criteria for Interactive Traffic

\begin{tabular}{|c|c|c|c|c|c|}
\hline Criteria & Delay & Jitter & PLR & Throughput & Weights \\
\hline \multirow{2}{*}{ Delay } & 1 & 4 & $1 / 2$ & 3 & 0.3200 \\
\hline Jitter & $(1,1,1)$ & $(1,1.5,2)$ & $(1,1.33,2)$ & $(0.667,1,1.5)$ & \\
\hline & $1 / 4$ & 1 & $1 / 8$ & $1 / 3$ & 0.0079 \\
\hline PLR & $(0.5,0.667,1)$ & $(1,1,1)$ & $(0.25,0.286,0.333)$ & $(0.667,1,1.5)$ & \\
\hline Throughput & 2 & 8 & 1 & 5 & 0.5475 \\
& $(0.5,0.75,1)$ & $(3,3.5,4)$ & $(1,1,1)$ & $(1.5,2,2.5)$ & \\
\hline & $(0.667,1,1.5)$ & $(0.667,1,1.5)$ & $(0.4,0.5,0.667)$ & $(1,1,1)$ & 0.1246 \\
\hline
\end{tabular}

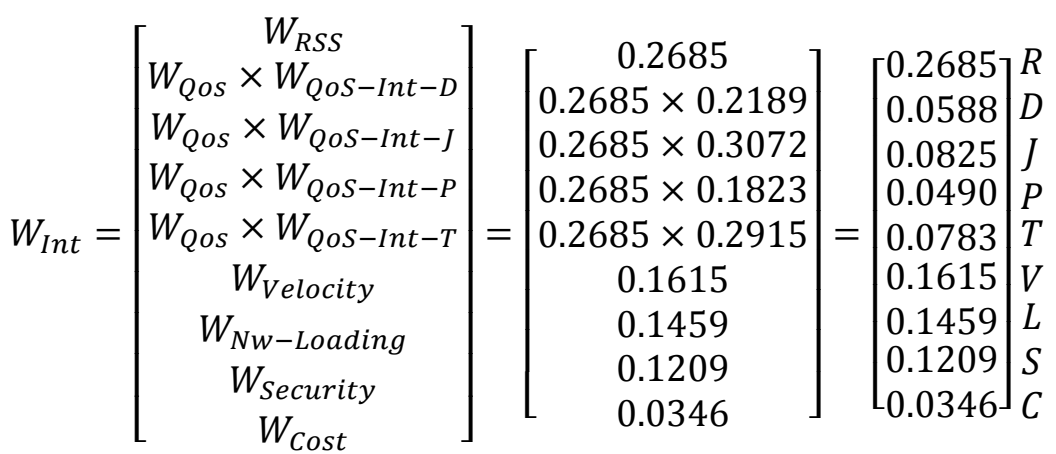

\subsection{Weights for Background Traffic Class}

FAHP decision matrix for level-2 criteria and the overall weightings for all the attributes for the Background traffic class are shown in Table 3.20, and in Equation (3.23), respectively.

$$
W_{\text {Back }}=\left[\begin{array}{c}
W_{\text {RSS }} \\
W_{\text {Qos }} \times W_{\text {QoS-Back-D }} \\
W_{\text {Qos }} \times W_{\text {QoS-Back-J }} \\
W_{\text {Qos }} \times W_{\text {QoS-Back-P }} \\
W_{\text {Qos }} \times W_{\text {QoS-Back-T }} \\
W_{\text {Velocity }} \\
W_{\text {Nw-Loading }} \\
W_{\text {Security }} \\
W_{\text {Cost }}
\end{array}\right]=\left[\begin{array}{c}
0.2685 \\
0.2685 \times 0.1653 \\
0.2685 \times 0.3134 \\
0.2685 \times 0.1048 \\
0.2685 \times 0.4165 \\
0.1615 \\
0.1459 \\
0.1209 \\
0.0346
\end{array}\right]=\left[\begin{array}{c}
0.2685 \\
0.0444 \\
0.0842 \\
0.0281 \\
0.1118 \\
0.1615 \\
D \\
D \\
D \\
0.1459 \\
0.1209 \\
0.0346
\end{array}\right] \text { S }
$$


Table 3.20: FAHP Decision Matrix for QoS Sub-criteria for Background Traffic

\begin{tabular}{|c|c|c|c|c|c|}
\hline Criteria & Delay & Jitter & PLR & Throughput & Weights \\
\hline Delay & $\begin{array}{c}1 \\
(1,1,1)\end{array}$ & $\begin{array}{c}1 \\
(1,1,1)\end{array}$ & $\begin{array}{c}1 / 4 \\
(0.5,0.667,1)\end{array}$ & $\begin{array}{c}1 / 2 \\
(1,1.33,2)\end{array}$ & 0.2416 \\
\hline Jitter & $\begin{array}{c}1 \\
(1,1,1)\end{array}$ & $\begin{array}{c}1 \\
(1,1,1)\end{array}$ & $\begin{array}{c}1 / 4 \\
(0.5,0.667,1)\end{array}$ & $\begin{array}{c}1 / 2 \\
(1,1.33,2)\end{array}$ & 0.2416 \\
\hline PLR & $\begin{array}{c}4 \\
(1,1.5,2)\end{array}$ & $\begin{array}{c}4 \\
(1,1.5,2)\end{array}$ & $\begin{array}{c}1 \\
(1,1,1)\end{array}$ & $\begin{array}{c}3 \\
(0.667,1,1.5)\end{array}$ & 0.3147 \\
\hline Throughput & $\begin{array}{c}2 \\
(0.5,0.75,1)\end{array}$ & $\begin{array}{c}2 \\
(0.5,0.75,1)\end{array}$ & $\begin{array}{c}3 \\
(0.667,1,1.5)\end{array}$ & $\begin{array}{c}1 \\
(1,1,1)\end{array}$ & 0.2020 \\
\hline
\end{tabular}

Table 3.21: FAHP Decision Matrix for QoS Sub-criteria for Streaming Traffic

\begin{tabular}{|c|c|c|c|c|c|}
\hline Criteria & Delay & Jitter & PLR & Throughput & Weights \\
\hline Delay & 1 & $1 / 3$ & $1 / 4$ & $1 / 5$ & 0.1767 \\
\hline Jitter & $(1,1,1)$ & $(0.667,1,1.5)$ & $(0.5,0.667,1)$ & $(0.4,0.5,0.667)$ & \\
\hline PLR & $(0.667,1,1.5)$ & $(1,1,1)$ & $(1,1.33,2)$ & $(0.667,1,1.5)$ & 0.2628 \\
\hline \multirow{2}{*}{ Throughput } & 4 & 2 & 1 & $1 / 2$ & 0.2756 \\
& $(1,1.5,2)$ & $(0.5,0.75,1)$ & $(1,1,1)$ & $(1,1.33,2)$ & \\
\hline & 5 & 3 & 2 & 1 & 0.2849 \\
\hline
\end{tabular}

\subsection{Weights for Streaming Traffic Class}

Table 3.21 shows FAHP decision matrix for level-2 criteria for the Streaming traffic class, and the overall weightings for all attributes are given in Equation (3.24).

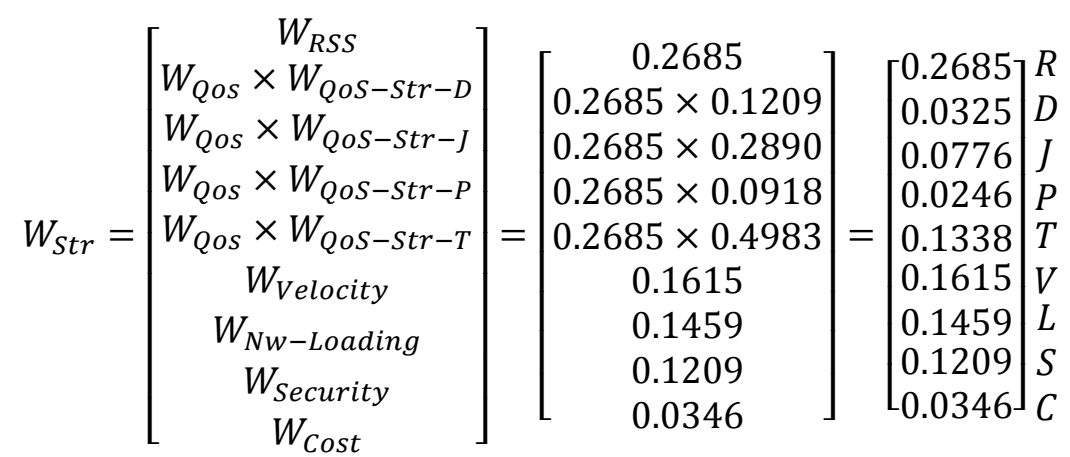




\subsubsection{Weights Calculations Using Linguistic Variables}

Linguistic Variables and TFNs have been extensively used in calculating the criteria weights in MADM problems [119-121]. Linguistic Variables are represented in Linguistic terms, whose values can be modeled using fuzzy sets. These Linguistic Variables have proven very useful when dealing with complex problems involving uncertainty. For the case of network selection, the uncertainty resides in the vague preferences specified by the end-users. This research work follows the same methodology proposed in [121], which is based on the usage of triangular fuzzy numbers representing Linguistic Variables. Using this approach, the TFNs can be transformed into crisp numbers via Equation (3.25), making the algorithm easier and more efficient. Table 3.22 shows TFNs and their corresponding crisp values for different Linguistic terms utilized in this research work.

$$
W(\tilde{A})=\frac{1}{6}(l+4 m+u)
$$

where $\tilde{A}=(l, m, u)$ represents a triangular fuzzy number.

The weights for each attribute, based on the different traffic classes, are calculated using the same steps outlined in Section 3.2.2.1. The only difference is the adaption and application of Linguistic Variables with triangular fuzzy numbers. Final weights are produced following a simple weighted sum normalization process. A summary of the calculated weights for all of the four traffic classes using this modified approach is provided as follows. 


\subsection{Weights for Conversational Traffic Class}

Tables 3.23-3.24 show assigned Linguistic Variables and their corresponding normalized weights for level-1 and level-2 criteria for the Conversational traffic class, respectively. The weights generated in Tables 3.25-3.27 are used to create the interdependence matrix of QoS parameters, given in Table 3.28. Weights for all the attributes for the Conversational traffic class are shown in Equation (3.26).

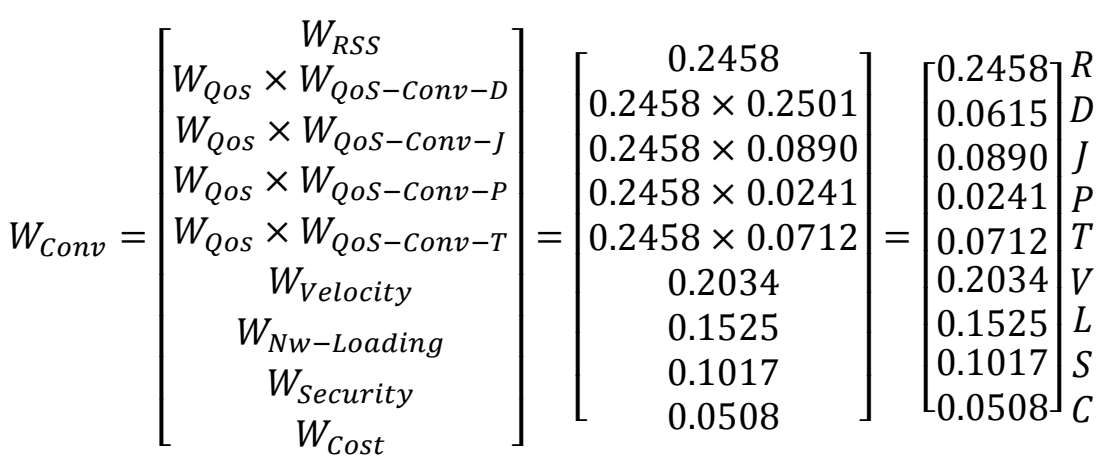

\subsection{Weights for Interactive Traffic Class}

Table 3.29 shows assigned Linguistic Variables and their corresponding normalized weights for level-2 criteria for the Interactive traffic class. The weights used for the Interactive traffic class are shown in Equation (3.27).

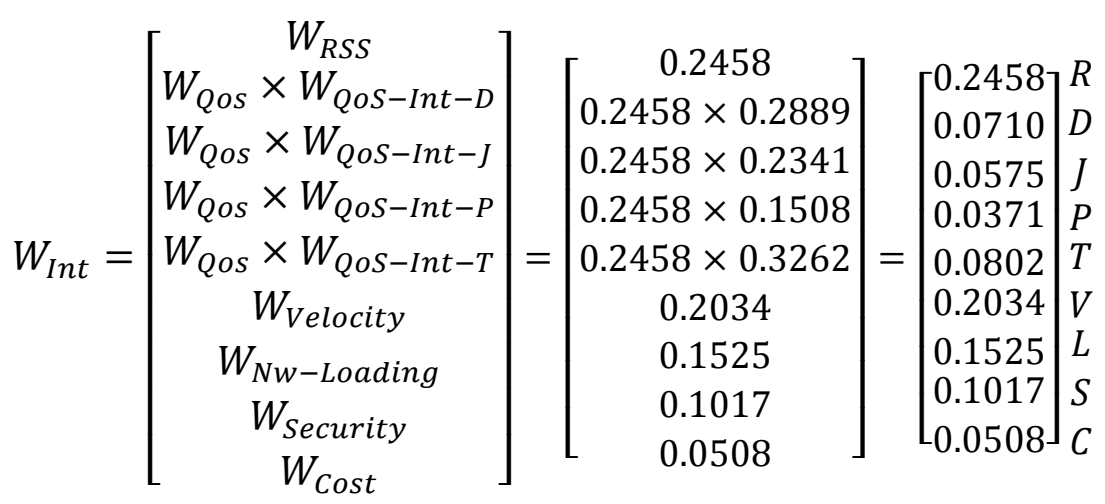


Table 3.22: Linguistic Variables with TFNs and Crisp Values

\begin{tabular}{|l|c|c|}
\hline Linguistic Variable & Triangular Fuzzy Number & Crisp Value \\
\hline Very Low (VL) & $(0.0,0.0,0.2)$ & 0.0333 \\
\hline Low (L) & $(0.0,0.2,0.4)$ & 0.2000 \\
\hline Medium (M) & $(0.2,0.4,0.6)$ & 0.4000 \\
\hline High (H) & $(0.4,0.6,0.8)$ & 0.6000 \\
\hline Very High (VH) & $(0.6,0.8,1.0)$ & 0.8000 \\
\hline Excellent (E) & $(0.8,1.0,1.0)$ & 0.9667 \\
\hline
\end{tabular}

Table 3.23: Linguistic Variables and Weights for Level-1 Criteria

\begin{tabular}{|c|c|c|}
\hline Criteria & Linguistic Variable & Normalized Weights \\
\hline RSS & E & 0.2458 \\
\hline QoS & E & 0.2458 \\
\hline Velocity & VH & 0.2034 \\
\hline Traffic Load & H & 0.1525 \\
\hline Security & M & 0.1017 \\
\hline Cost & L & 0.0508 \\
\hline
\end{tabular}

Table 3.24: Linguistic Variables and Weights for QoS criteria, Conversational Traffic

\begin{tabular}{|c|c|c|}
\hline Criteria & Linguistic Variable & Normalized Weight \\
\hline Delay & E & 0.3537 \\
\hline Jitter & E & 0.3537 \\
\hline PLR & H & 0.2195 \\
\hline Throughput & L & 0.0732 \\
\hline
\end{tabular}


Table 3.25: Linguistic Variables and Weights w.r.t Delay

\begin{tabular}{|c|c|c|}
\hline Criteria & Linguistic Variable & Normalized Weight \\
\hline Delay & $\mathrm{E}$ & 0.7073 \\
\hline Jitter & $\mathrm{M}$ & 0.2927 \\
\hline
\end{tabular}

Table 3.26: Linguistic Variables and Weights w.r.t Jitter

\begin{tabular}{|c|c|c|}
\hline Criteria & Linguistic Variable & Normalized Weights \\
\hline Jitter & $\mathrm{E}$ & 0.6170 \\
\hline Throughput & $\mathrm{H}$ & 0.3830 \\
\hline
\end{tabular}

Table 3.27: Linguistic Variables and Weights w.r.t PLR

\begin{tabular}{|c|c|c|}
\hline Criteria & Linguistic Variable & Normalized Weights \\
\hline Jitter & M & 0.1846 \\
\hline PLR & E & 0.4462 \\
\hline Throughput & VH & 0.3692 \\
\hline
\end{tabular}

Table 3.28: Linguistic Variables Interdependence Matrix for QoS parameters

\begin{tabular}{|c|c|c|c|c|}
\hline Criteria & Delay & Jitter & PLR & Throughput \\
\hline Delay & 0.7073 & 0.0000 & 0.0000 & 0.0000 \\
\hline Jitter & 0.2927 & 0.6170 & 0.1846 & 0.0000 \\
\hline PLR & 0.0000 & 0.0000 & 0.4462 & 0.0000 \\
\hline Throughput & 0.0000 & 0.3830 & 0.3692 & 1.0000 \\
\hline
\end{tabular}

Table 3.29: Linguistic Variables \& Weights for QoS Sub-criteria, Interactive Traffic

\begin{tabular}{|c|c|c|}
\hline Criteria & Linguistic Variable & Normalized Weight \\
\hline Delay & E & 0.4085 \\
\hline Jitter & L & 0.0845 \\
\hline PLR & VH & 0.3380 \\
\hline Throughput & M & 0.1690 \\
\hline
\end{tabular}


Table 3.30: Linguistic Variables \& Weights for QoS Sub-criteria, Background Traffic

\begin{tabular}{|c|c|c|}
\hline Criteria & Linguistic Variable & Normalized Weight \\
\hline Delay & L & 0.1132 \\
\hline Jitter & L & 0.1132 \\
\hline PLR & M & 0.2264 \\
\hline Throughput & $\mathrm{E}$ & 0.5472 \\
\hline
\end{tabular}

\subsection{Weights for Background Traffic Class}

Table 3.30 shows Linguistic Variables and the corresponding weights for level-2 criteria for the Background traffic class, and Equation (3.28) gives the overall weightings for this traffic class.

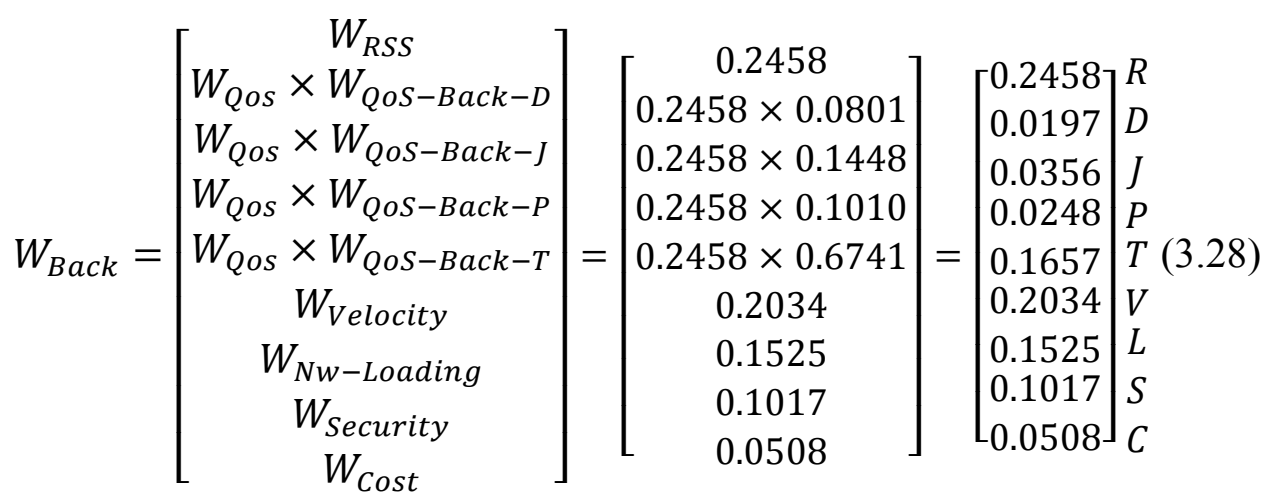

\subsection{Weights for Streaming Traffic Class}

The Linguistic Variables and their corresponding normalized weights for level-2 criteria for the Streaming traffic class are shown in Table 3.31. Equation (3.29) displays the weights of all the attributes for the Streaming traffic class. 
Table 3.31: Linguistic Variables \& Weights for QoS criteria, Streaming Traffic

\begin{tabular}{|c|c|c|}
\hline Criteria & Linguistic Variable & Normalized Weight \\
\hline Delay & L & 0.0779 \\
\hline Jitter & H & 0.2338 \\
\hline PLR & VH & 0.3117 \\
\hline Throughput & E & 0.3766 \\
\hline
\end{tabular}

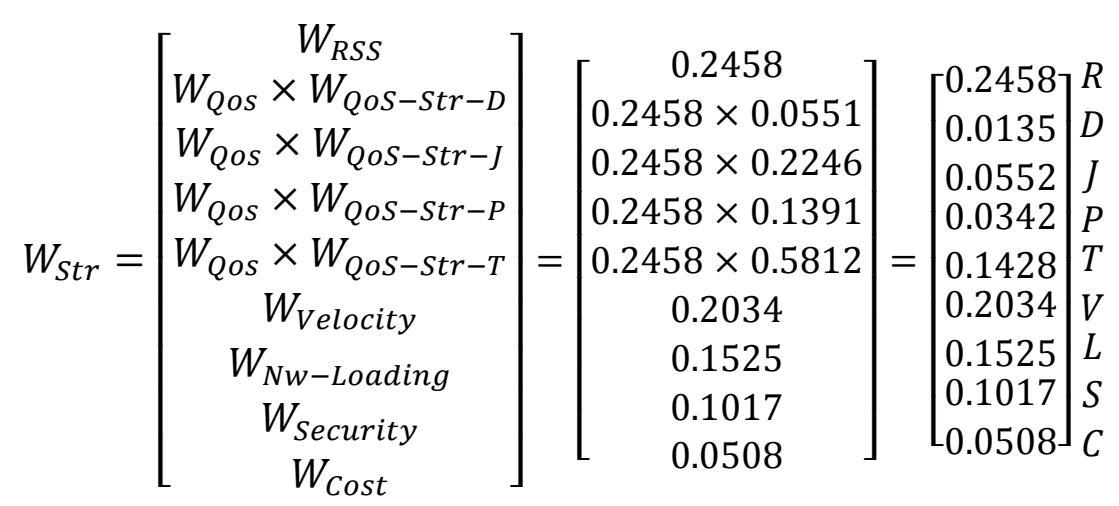

\subsubsection{BRIEF COMPARISON OF WEIGHTS CALCULATIONS SCHEMES}

For the convenience, Table 3.32 shows the weights for different attributes for all four traffic classes calculated using the three different schemes provided in the previous sections.

\subsubsection{RSS PREDICTION USING GREY PREDICTION THEORY}

Although RSS, with threshold and hysteresis approaches [107], can minimize the number of unnecessary handoffs, these schemes result in a low data rate and high dropping probabilities since at the time of handoffs, the RSS reception from the current PoA may become too weak. Grey Prediction [122] based technique is used in [123] to predict values of RSS with threshold and hysteresis to perform horizontal handoffs. 
Table 3.32: Comparison of Different Weighting Schemes

\begin{tabular}{|c|c|c|c|c|c|c|c|c|c|c|}
\hline & \multirow{2}{*}{ RSS } & \multicolumn{4}{|c|}{ QoS } & \multirow{2}{*}{ Velocity } & \multirow{2}{*}{ Loading } & \multirow{2}{*}{ Security } & \multirow{2}{*}{ Cost } \\
\hline & & & D & $\mathrm{J}$ & P & $\mathrm{T}$ & & & & \\
\hline \multirow{4}{*}{ AHP } & Conv & 0.3271 & 0.1077 & 0.1228 & 0.0307 & 0.0660 & 0.1466 & 0.1087 & 0.0591 & 0.0314 \\
\hline & Str & 0.3271 & 0.0136 & 0.0573 & 0.0561 & 0.2001 & 0.1466 & 0.1087 & 0.0591 & 0.0314 \\
\hline & Int & 0.3271 & 0.0850 & 0.0428 & 0.0987 & 0.1005 & 0.1466 & 0.1087 & 0.0591 & 0.0314 \\
\hline & Back & 0.3271 & 0.0163 & 0.0342 & 0.1163 & 0.1604 & 0.1466 & 0.1087 & 0.0591 & 0.0314 \\
\hline \multirow{4}{*}{ FAHP } & Conv & 0.2685 & 0.0779 & 0.1024 & 0.0211 & 0.0672 & 0.1615 & 0.1459 & 0.1209 & 0.0346 \\
\hline & Str & 0.2685 & 0.0325 & 0.0776 & 0.0246 & 0.1338 & 0.1615 & 0.1459 & 0.1209 & 0.0346 \\
\hline & Int & 0.2685 & 0.0588 & 0.0825 & 0.0490 & 0.0783 & 0.1615 & 0.1459 & 0.1209 & 0.0346 \\
\hline & Back & 0.2685 & 0.0444 & 0.0842 & 0.0281 & 0.1118 & 0.1615 & 0.1459 & 0.1209 & 0.0346 \\
\hline \multirow{4}{*}{ TFN } & Conv & 0.2458 & 0.0615 & 0.0890 & 0.0241 & 0.0712 & 0.2034 & 0.1525 & 0.1017 & 0.0508 \\
\hline & Str & 0.2458 & 0.0135 & 0.0552 & 0.0342 & 0.1428 & 0.2034 & 0.1525 & 0.1017 & 0.0508 \\
\hline & Int & 0.2458 & 0.0710 & 0.0575 & 0.0371 & 0.0802 & 0.2034 & 0.1525 & 0.1017 & 0.0508 \\
\hline & Back & 0.2458 & 0.0197 & 0.0356 & 0.0248 & 0.1657 & 0.2034 & 0.1525 & 0.1017 & 0.0508 \\
\hline
\end{tabular}

In order to reduce the call dropping probability under a lognormal fading heterogeneous wireless environment, the proposed scheme utilizes predicted RSS values measured from the current PoA, as well as from the target networks. These predicted values, obtained using GPT, are utilized by the proposed scheme to determine if a future handoff is necessary or not. A MATLAB module is implemented to perform the above task.

In Grey theory, system dynamic model can be represented by $\operatorname{GM}(n, h)$, where $n$ is the order of Grey differential equation, and $h$ defines the number of variables. This research work utilizes one of the most popular and widely used Grey prediction models; 
the $\operatorname{GM}(1,1)$ model takes a sequence of $n$ RSS samples, which is given as $X^{(0)}=$ $\left\{x^{(0)}(1), x^{(0)}(2), \ldots, x^{(0)}(n)\right\}$. The Accumulated Generating Operation (AGO) is utilized to further process these samples due to the possible presence of random noise. The AGO operation produces a first-order AGO sequence that is given in Equation (3.30).

$$
x^{(1)}(k)=\sum_{i=1}^{k} x^{(0)}(i), \quad k=1,2, \ldots, n
$$

A linear dynamic model given in Equation (3.31) is then used to approximate the sequence that is obtained in Equation (3.26).

$$
x^{(0)}(k)+a x^{(1)}(k)=b
$$

where $a$ (developed parameter) and $b$ (grey input), which can be calculated using the least square approximation, are the coefficients of the differential equation whose solution is given in Equation (3.32).

$$
x^{(1)}(n+1)=\left[x^{(0)}(1)-\frac{b}{a}\right] e^{-a n}+\frac{b}{a}
$$

The vector representation of the parameters $a$ and $b$ is given by Equation (3.33).

$$
c=[a b]^{T}=\left(B^{T} B\right)^{-1} B^{T} \gamma_{n}
$$

where

$$
B=\left[\begin{array}{cc}
-\frac{1}{2}\left[x^{(1)}(1)+x^{(1)}(2)\right] & 1 \\
-\frac{1}{2}\left[x^{(1)}(2)+x^{(1)}(3)\right] & 1 \\
\vdots & \vdots \\
-\frac{1}{2}\left[x^{(1)}(n-1)+x^{(1)}(n)\right] & 1
\end{array}\right]
$$

and

$$
\gamma_{n}=\left[x^{(0)}(2), x^{(0)}(3), \ldots, x^{(0)}(n)\right]^{T}
$$


Table 3.33: Predicted Values of RSS using GPT for Different Network Types

\begin{tabular}{|c|c|c|}
\hline Network Type & RSSs Samples $(\mathbf{d b m})$ & PRSS (dbm) \\
\hline WLAN & {$[-110-110-112-113]$} & -114.69 \\
\hline WMAN & {$[-140-150-151-155]$} & -157.08 \\
\hline WWAN & {$[-110-111-100-95]$} & -86.84 \\
\hline
\end{tabular}

Thus the predicted value of RSS can be obtained by using Equation (3.34).

$$
\tilde{x}^{(0)}(n+1)=\left[x^{(0)}(1)-\frac{b}{a}\right] e^{-a n}\left(1-e^{a}\right)
$$

Table 3.33 shows RSS samples measured from different types of networks and their predicted values using GPT. While a continuous drop pattern for RSS can be observed for both WLAN and WMAN networks, results calculated using GPT could help reduce the unnecessary call drops due to the predicted value of weak RSS.

\subsubsection{NORMALIZATION OF ATTRIBUTES USING FUZZY TECHNIQUES}

A heterogeneous wireless network typically comprises of different types of wireless access technologies with dissimilar operating parameters and characteristics. In general, these dissimilar parameters are not directly comparable. For example, the RSS ranges of WLAN and WMAN are quite different. Therefore, a high value of RSS measured from a WLAN may not be considered high in a WMAN environment. Thus Fuzzy Logic is utilized to normalize these parameters in the range of $[0,1]$.

Fuzzy sets for each parameter are created based on the different network types. The Universe of Discourses (UoDs), for the input parameters, are selected based on the published standards for different network types (IEEE 802.11, IEEE 802.16, UMTS) [61, 
$108]$ and include the lowest and the highest values of the parameter that can be measured at the MS. Different Linguistic Variables such as low, medium, and high are created to partition these UoDs. Typical operating ranges of the attributes for three types of networks, utilized in this work are given in Table 3.34. For security and cost, we use a range of $[1,10], 10$ being the highest level of security provided and the most expensive network utilized.

Figures 3.4-3.6 show the fuzzy sets representing RSS of WWAN, WMAN, and WLAN, respectively. Due to their computational simplicity, trapezoidal membership functions, as defined in Equation (3.35), are used.

$$
\mu_{A}(x)=\left\{\begin{array}{cc}
0 & x<l ; x>u \\
\frac{x-l}{c-\frac{w}{2}-l} & l<x<\left(c-\frac{w}{2}\right) \\
\frac{u-x}{u-\frac{w}{2}-c} & \left(c+\frac{w}{2}\right)<x<u \\
1 & \left(c-\frac{w}{2}\right)<x<\left(c+\frac{w}{2}\right)
\end{array}\right\}
$$

where $l$, and $u$ are the lower and upper bounds respectively, $c$ is the center and $w$ is the width of the top side of the symmetric trapezoid.

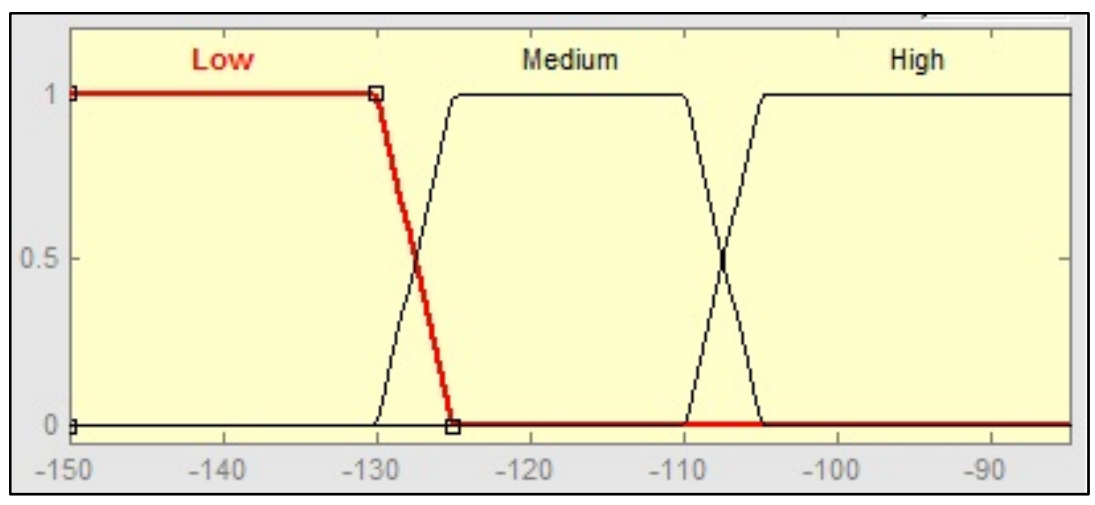

Figure 3.4: Fuzzy Set Representing RSS (WWAN) 


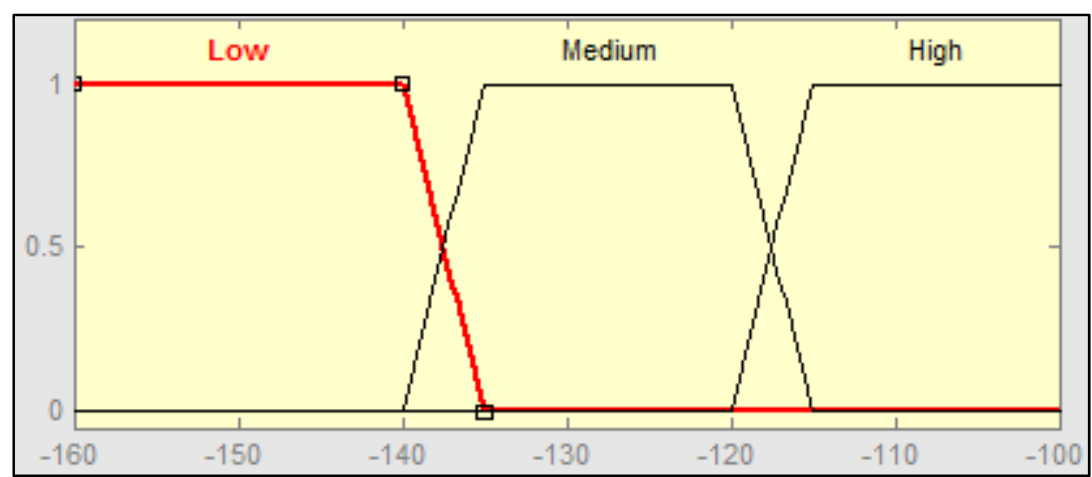

Figure 3.5: Fuzzy Set Representing RSS (WMAN)

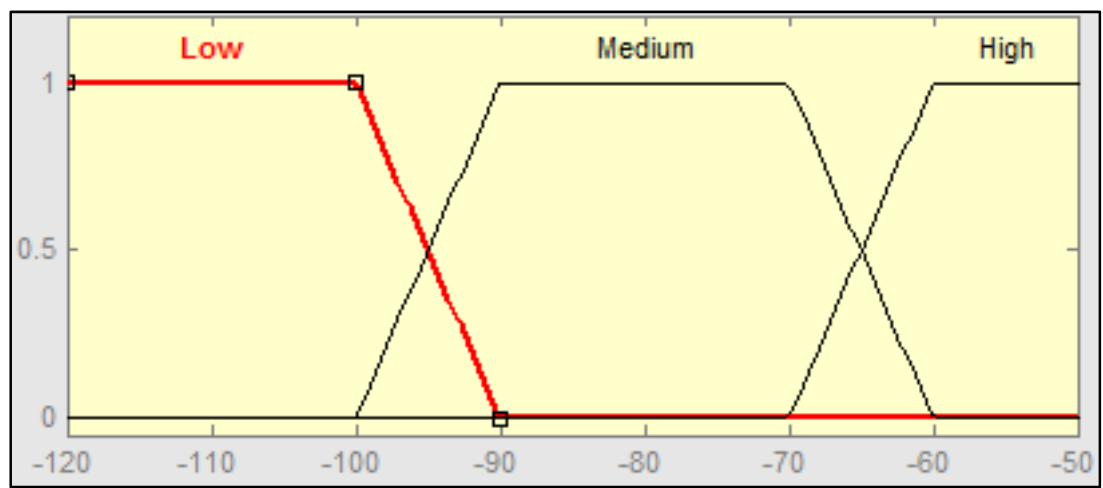

Figure 3.6: Fuzzy Set Representing RSS (WLAN)

Table 3.34: Parameter Ranges for Different Network Types

\begin{tabular}{|l|c|c|c|}
\hline & WLAN & WMAN & WWAN \\
\hline RSS (dbm) & $-110--55$ & $-160--100$ & $-150--90$ \\
\hline Delay (ms) & $100-150$ & $10-50$ & $10-75$ \\
\hline Jitter (ms) & $10-30$ & $3-12$ & $5-15$ \\
\hline PLR per $\mathbf{1 0}^{6}$ bytes (\%) & $3-7$ & $1-8$ & $1-5$ \\
\hline Throughput (Mbps) & $50-150$ & $20-100$ & $0.1-3$ \\
\hline Network Range (m) & $0-100$ & $0-350$ & $0-750$ \\
\hline Velocity (mps) & & $0-10$ & \\
\hline Traffic Load (\%) & & $0-100$ & \\
\hline Security & & $1-10$ & \\
\hline Cost & & & \\
\hline
\end{tabular}


Several parallel FLCs with reduced rule-sets are created to normalize network parameters. These network parameters can be classified as benefit type or cost type. For example, RSS is a benefit type attribute, as highest possible value of RSS is desired from a given network. On the other hand, network delay, which is preferred to be a minimal value, is characterized as a cost type attribute. The normalization of both classes of parameters is performed using specific fuzzy rules in such a way that the respective FIS produces high membership values for benefit type parameters and low membership values for the cost types. Figures 3.7-3.8 show FLCs for RSS (measured from WMAN) and the respective rule-set, whereas Figures 3.9-3.10 show the same for cost type parameter, latency. Since RSS is a benefit type parameter, inference rules are used to find the probability of the MS selecting WMAN based on the measured RSS. On the other hand, the probability of the MS rejecting WMAN is calculated based on the measured value of delay, a cost type parameter.

Other system parameters, such as throughput and Security preference, are considered as benefit type attributes, whereas jitter, PLR, velocity, traffic-load, and network cost are classified as cost type attributes. The calculated membership values of all these parameters are utilized in the future steps of our proposed scheme.

\subsubsection{CALCULATION OF DEGREE OF QoS FOR CURRENT PoA}

In order to maximize the end-user's satisfaction, the QoS, as provided by the current PoA (serving network), for the existing service (based on the traffic class) is calculated, monitored, and compared using a fuzzy inference system (discussed in the next section). 


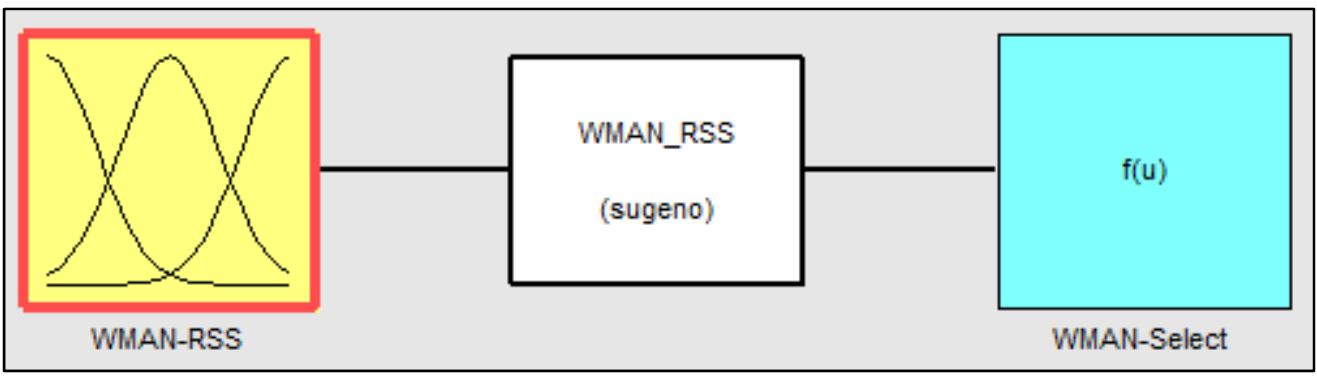

Figure 3.7: FLC for RSS (WMAN)

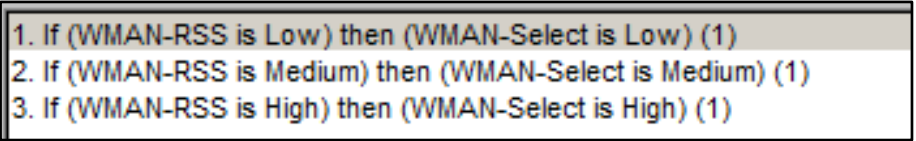

Figure 3.8: Fuzzy Rule-set for RSS (WMAN)

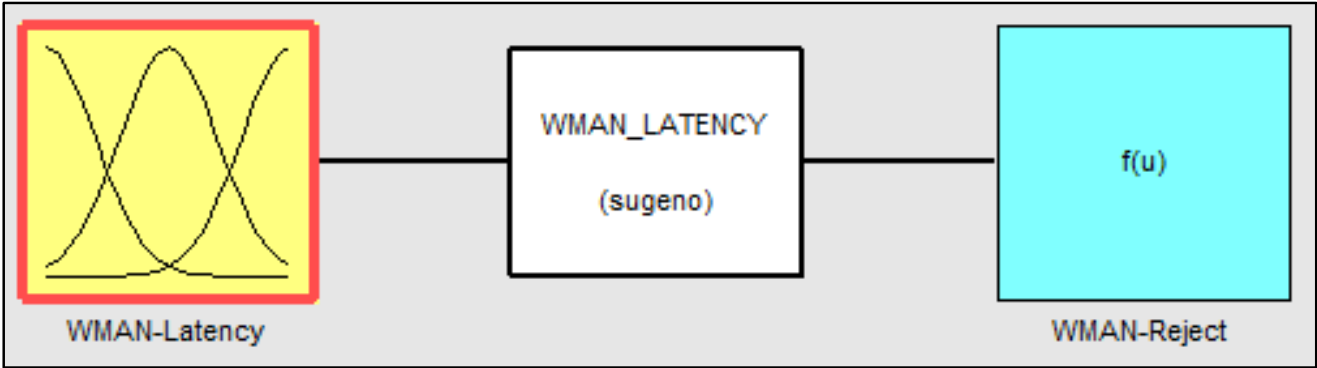

Figure 3.9: FLC for Latency (WMAN)

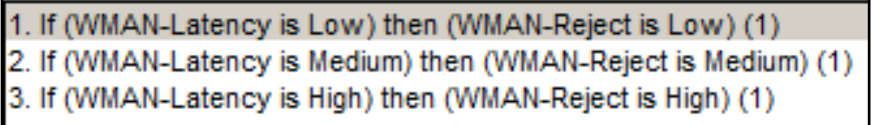

Figure 3.10: Fuzzy Rule-set for Latency (WMAN)

The degree of QoS is calculated in the range of $[0,1]$ by performing a weighted sum of the membership values of delay, jitter, PLR, and throughput, which are obtained in the previous section. This weighted sum is given in Equation (3.36).

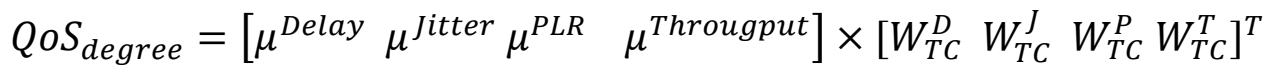


where $W_{T C}^{X}$ is the weight of the QoS parameter $X$ (delay, jitter, PLR, and throughput) for a particular traffic class TC (Conversational, Streaming, Background, and Interactive). These weights are calculated using different mechanisms as discussed in Section 3.2.2.

The degree of QoS that is obtained in this step serves as one of the inputs to the fuzzy inference system that is defined next.

\subsubsection{VHO FACTOR CALCULATION USING FUZZY LOGIC}

In our proposed scheme, we utilize four different fuzzy logic controllers to calculate the value of Vertical Handoff Factor and determine the necessity of handoffs based on the current conditions of serving PoA. In order to reduce the number of rules and system complexity, three fuzzy logic controllers are combined in a parallel fashion. The outputs of these three FLCs are then fed into the fourth fuzzy logic controller that produces the final VHO factor. Both Sugeno [124] and Mamdani [125] type FISs are incorporated with carefully designed rules. Figure 3.11 shows the overall design of these FLCs that are used to determine the necessity of VHO. In the following subsections, the details of these four fuzzy logic controllers are provided.

\subsubsection{Design of Fuzzy Logic Controller 1}

Velocity is considered an important factor in the proposed scheme. To reduce the call dropping probability and the unnecessary handoffs, MSs with higher speeds should be connected to networks with larger coverage areas; for example, WWAN. Figures 3.123.13 show the design for FLC-1 and the membership function for the input variable (MSVelocity), respectively. A Sugeno based FIS with one input (MS-Velocity) and three output variables are utilized. The input variable has three membership functions (Low, 
Medium, and High) and each of the three output variables has three singleton membership functions (Low, Medium, and High). The output variables reflect the probability of rejection for an MS to be in a specific network type at a given input speed.

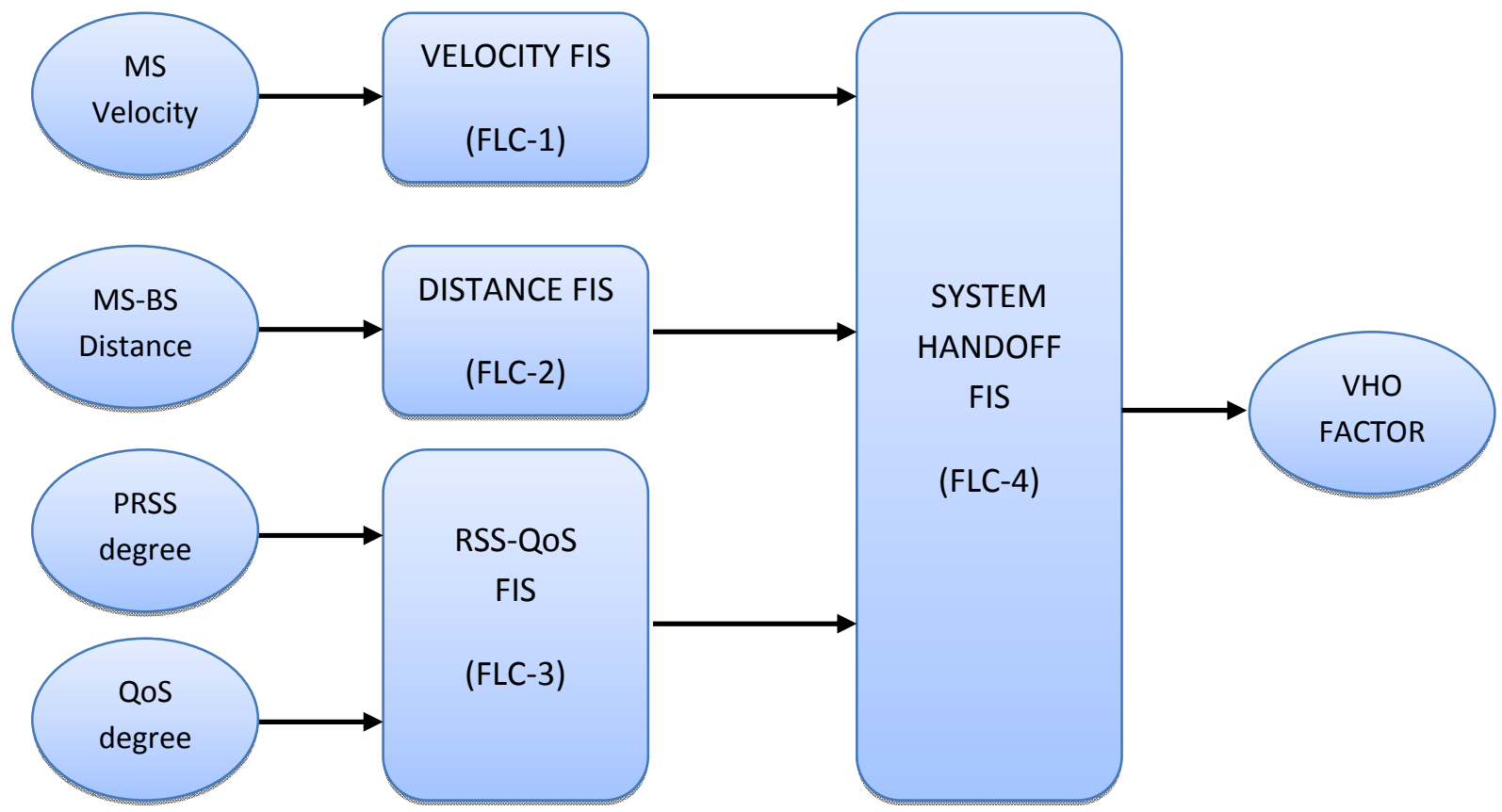

Figure 3.11: Fuzzy Logic Controllers for VHO Necessity Estimation

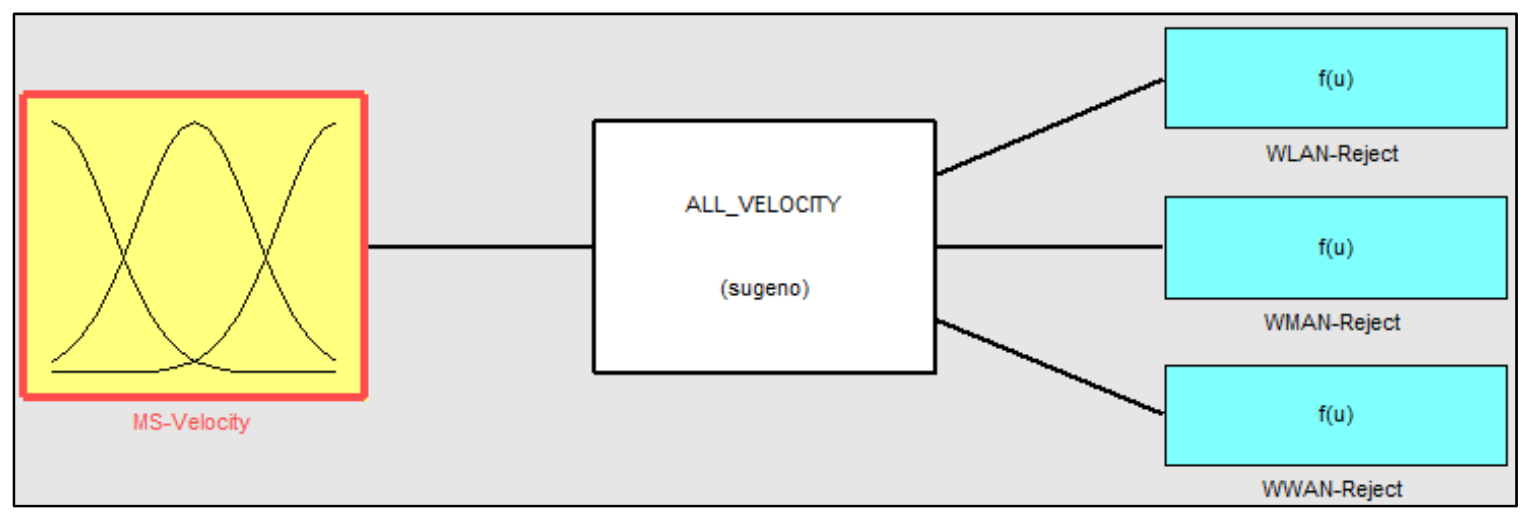

Figure 3.12: Design for FLC-1 


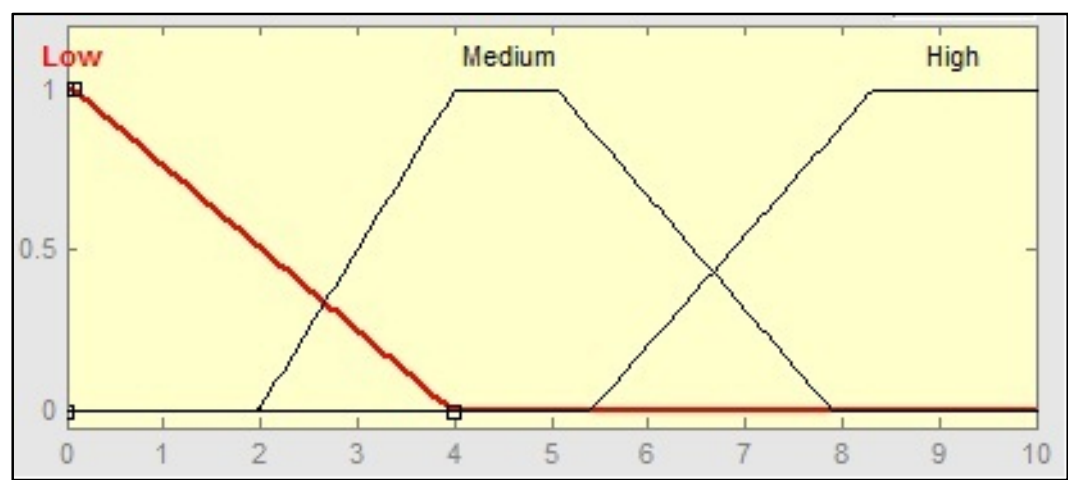

Figure 3.13: Membership Function for MS-Velocity for FLC-1

\section{If (MS-Velocity is Low) then (WLAN-Reject is Low)(WMAN-Reject is Medium)(WWAN-Reject is High) (1) \\ 2. If (MS-Velocity is High) then (WLAN-Reject is High)(WMAN-Reject is High)(WWAN-Reject is Low) (1) \\ 3. If (MS-Velocity is Medium) then (WLAN-Reject is High)(WMAN-Reject is Low)(WWAN-Reject is Medium) (1)}

Figure 3.14: Inference Rules for FLC-1

Inference rules, as shown in Figure 3.14, are designed with the objective of connecting the higher speed MSs to a network with larger coverage area.

Figure 3.15 shows the control surface for the output variable WWAN-Reject. It is clear from the figure that the probability of rejecting WWAN network at higher speed is very low.

Figure 3.16 shows the evaluation of rules for FLC-1 with an MS moving at a speed of 7.5 meters per second. It can be observed from this figure that the probabilities of rejections for an MS to be in WLAN, WMAN, and WWAN are 0.95, 0.803, and 0.132, respectively. 


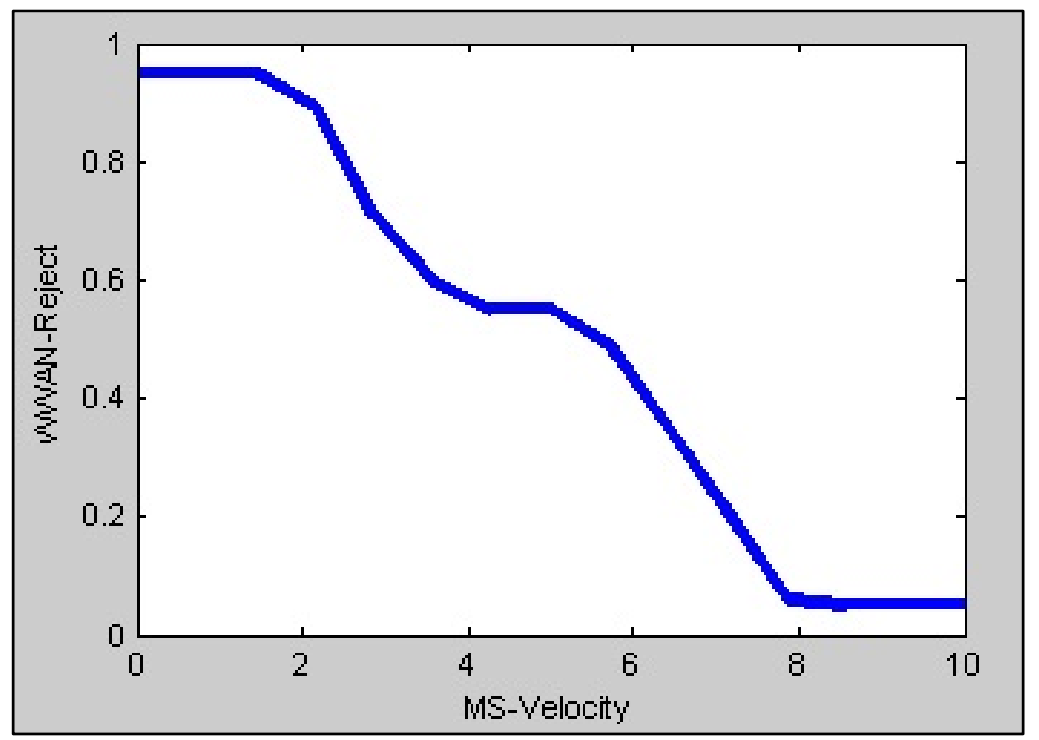

Figure 3.15: The Control Surface of the Output Variable WWAN-Reject for FLC-1

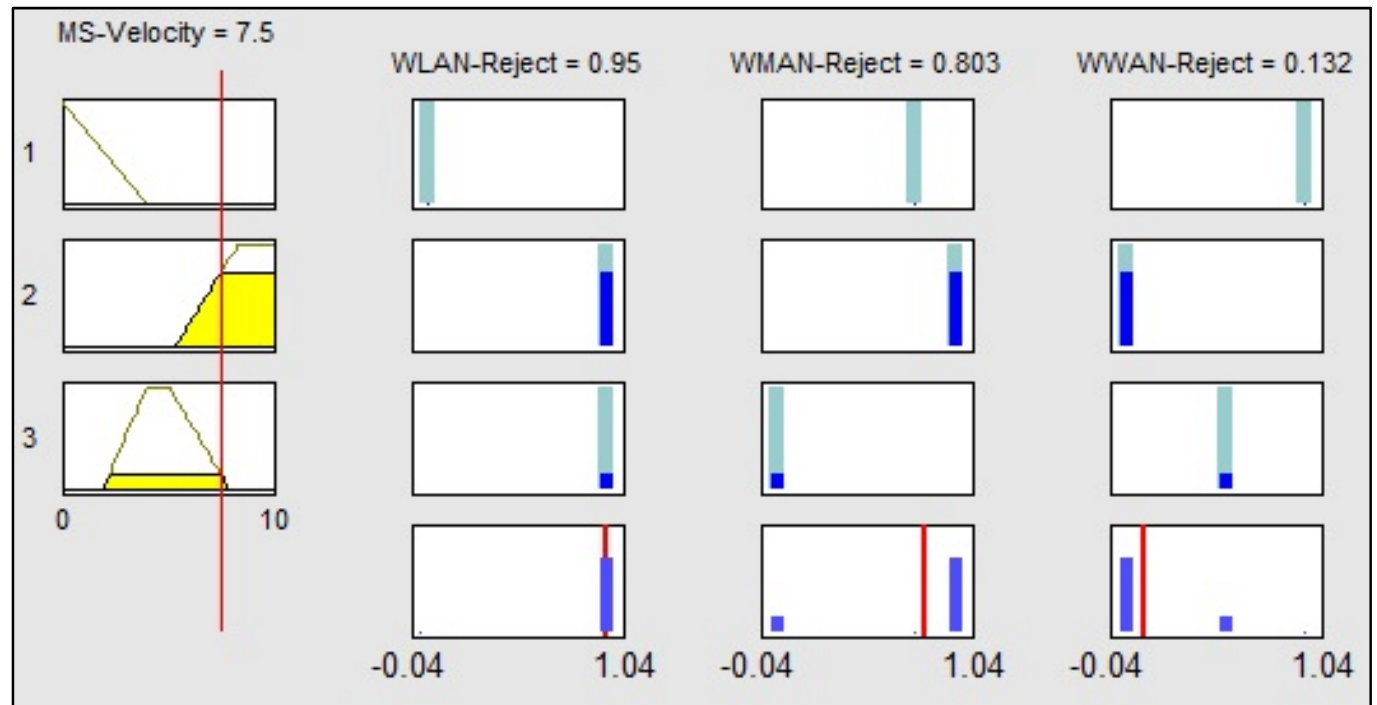

Figure 3.16: Rules Evaluation for FLC-1

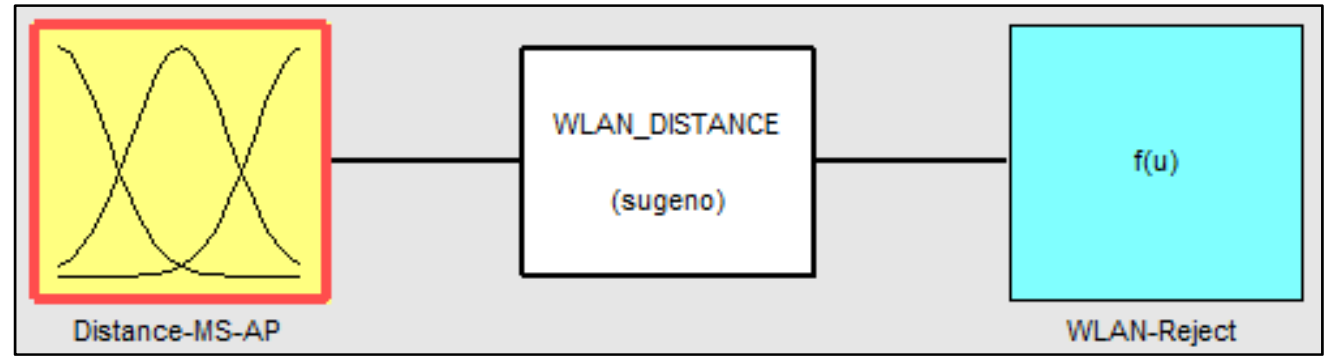

Figure 3.17: Design of FLC-2 (WLAN) 


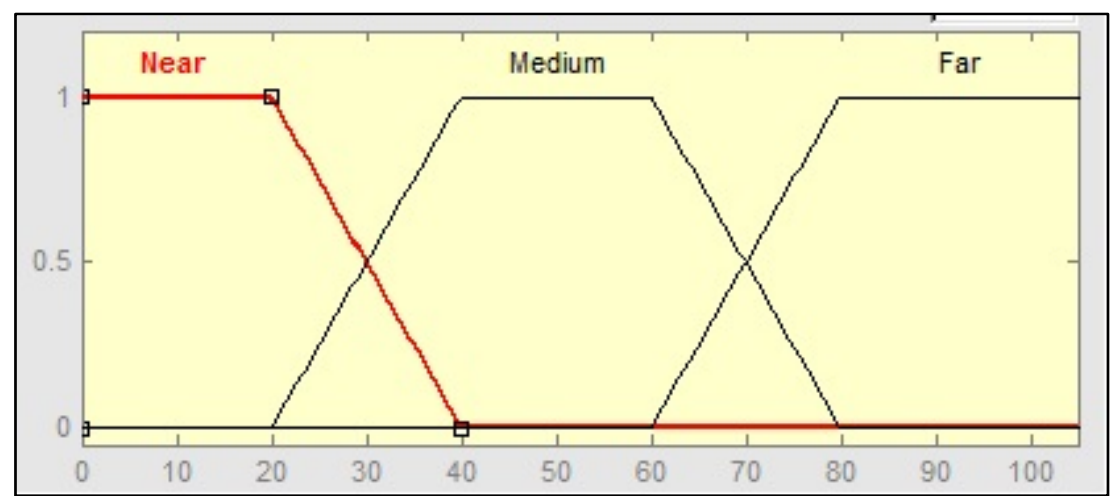

Figure 3.18: Membership Functions for FLC-2 (WLAN)

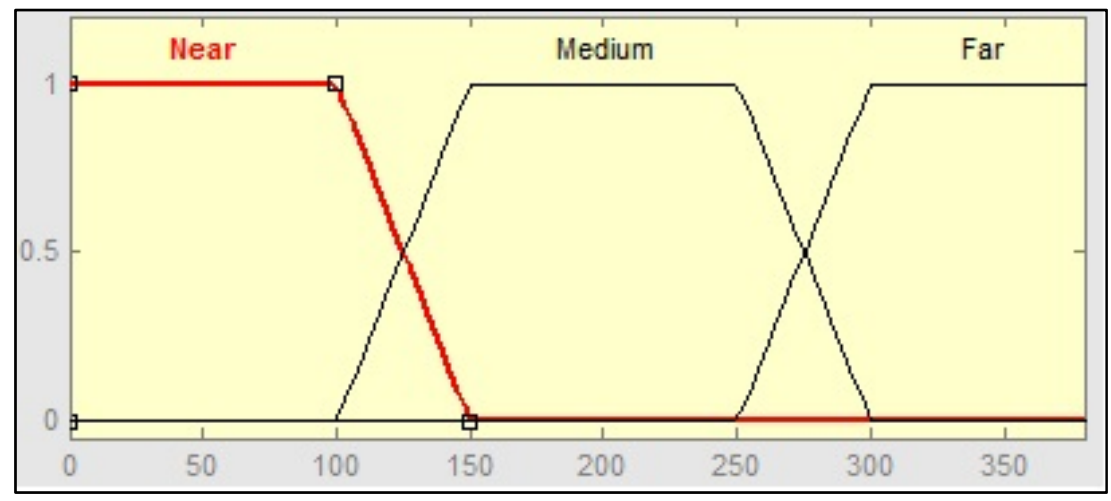

Figure 3.19: Membership Functions for FLC-2 (WMAN)

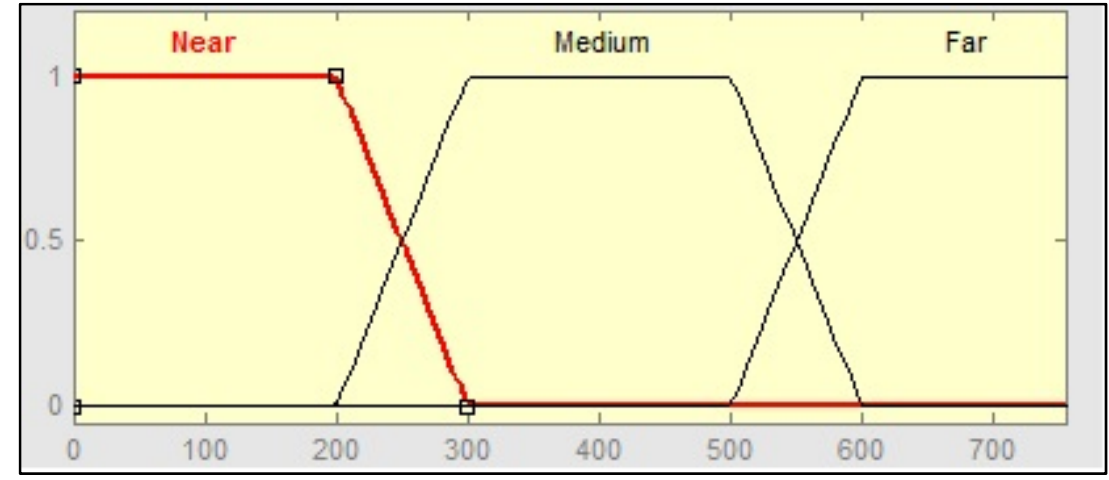

Figure 3.20: Membership Functions for FLC-2 (WWAN) 
1. If (Distance-MS-AP is Far) then (WLAN-Reject is High) (1)

2. If (Distance-MS-AP is Medium) then (WLAN-Reject is High) (1)

3. If (Distance-MS-AP is Near) then (WLAN-Reject is Low) (1)

Figure 3.21: Inference Rules for FLC-2 (WLAN)

1. If (Distance-MS-AP is Far) then (WMAN-Reject is High) (1)

2. If (Distance-MS-AP is Medium) then (WMAN-Reject is Medium) (1)

3. If (Distance-MS-AP is Near) then (WMAN-Reject is Low) (1)

Figure 3.22: Inference Rules for FLC-2 (WMAN)

1. If (Distance-MS-AP is Far) then (WWAN-Reject is High) (1)

2. If (Distance-MS-AP is Medium) then (WWAN-Reject is Low) (1)

3. If (Distance-MS-AP is Near) then (WWAN-Reject is Low) (1)

Figure 3.23: Inference Rules for FLC-2 (WWAN)

\subsubsection{Design of Fuzzy Logic Controller 2}

Distance between the MS and the current PoA (BS/AP) also plays a critical role in determining the necessity of handoffs. As the distance between the MS and the current PoA increases, the measured values of RSS and other critical factors decrease. Thus, handoff becomes imminent. The FLC-2 in the proposed scheme is designed based on the coverage provided by a specific network type. Since the coverage area of the three types of networks are different, and the assumption that at the most MS will be connected to one PoA, separate membership functions with different UoDs are designed based on these network types. Figure 3.17 shows the FLC-2 design for WLAN. A Sugeno based FIS with one input (Distance between MS and AP) and one output (probability of rejection in WLAN) is created. A similar design for WMAN and WWAN is followed. Figures 3.18-3.20 show the membership functions representing distance for WLAN, WMAN, and WWAN, respectively. Figures 3.21-3.23 show the inference rules designed for FLC-2 for the three network types. Figure 3.24 shows the control surface for the 
output variable WMAN-Reject and Figure 3.25 shows the rule evaluations for FLC-2 for a WMAN network.

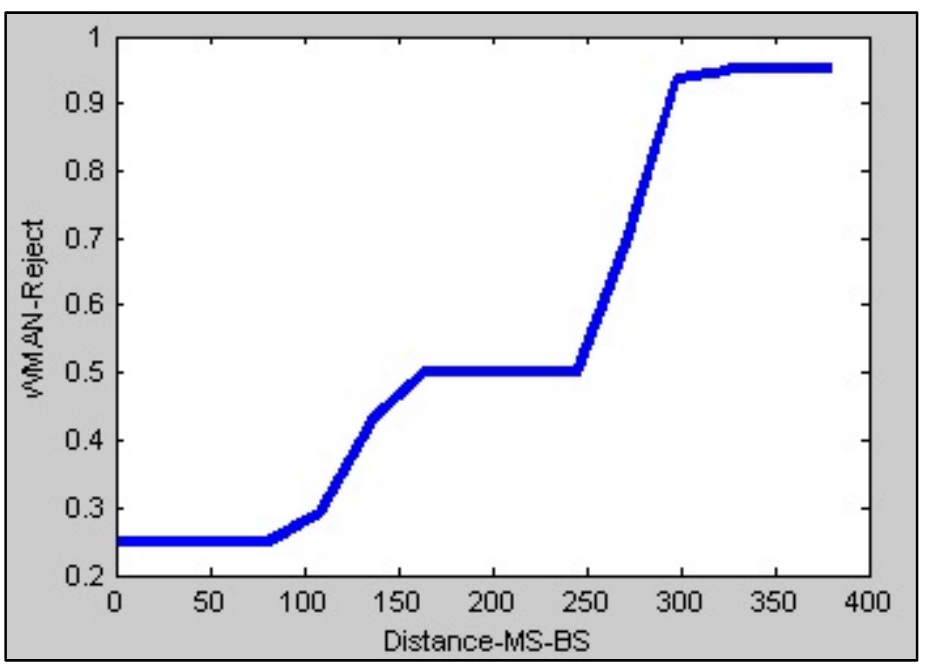

Figure 3.24: The Control Surface of the Output Variable WMAN-Reject for FLC-2

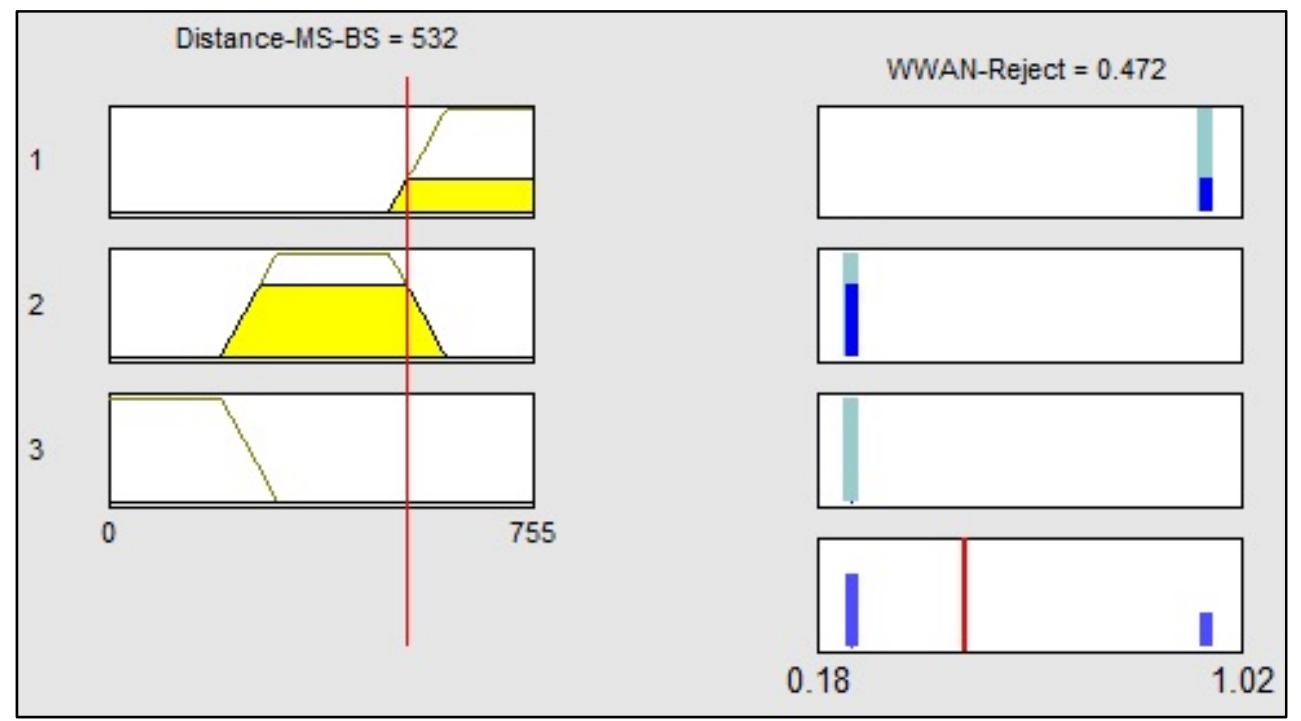

Figure 3.25: Rules Evaluation for FLC-2 (WWAN) 


\subsubsection{Design of Fuzzy Logic Controller 3}

The main objective of the proposed scheme has been to maximize the end-user's satisfaction in terms of the quality and continuity of the currently utilized service. RSS and QoS play a very important role in achieving this objective. If any of these two parameters fall below a threshold, the overall quality of the current application session diminishes resulting in reduced end-user's satisfaction levels. The FLC-3 is designed to make sure that the MS performs handoff before any of these two factors fall below the minimum network values required to sustain the quality of the currently utilized service.

Since the QoS measurements vary from one network type to another, separate FLCs are designed for each of the three network types. Figure 3.26 shows the design of FLC-3 for WLAN. A Mamdani based FIS with two inputs and one output is utilized. The inputs, WLAN-PRSS-degree and WLAN-QoS-degree, calculated in Sections 3.2.5 and 3.2.6, respectively, represent the quality of the received signal measured at the MS and the quality of the currently utilized service. Figures 3.27-3.28 show the trapezoidal membership functions for these input variables, respectively. The inference rules for FLC-3 for WLAN, as shown in Figure 3.29, indicate that a low value of both inputs results in a high value for the output variable, PRSS-QoS-Factor, indicating a handoff possibility. Figure 3.30 shows the control surface area for the output variable PRSS-QoSFactor for FLC-3 that is designed for WLAN. Figures 3.31-3.32 show two different instances with low and high values of the two input variables. After evaluating the inference rules, a high value of the output variable, indicating high handoff probability, can be observed from these figures. 


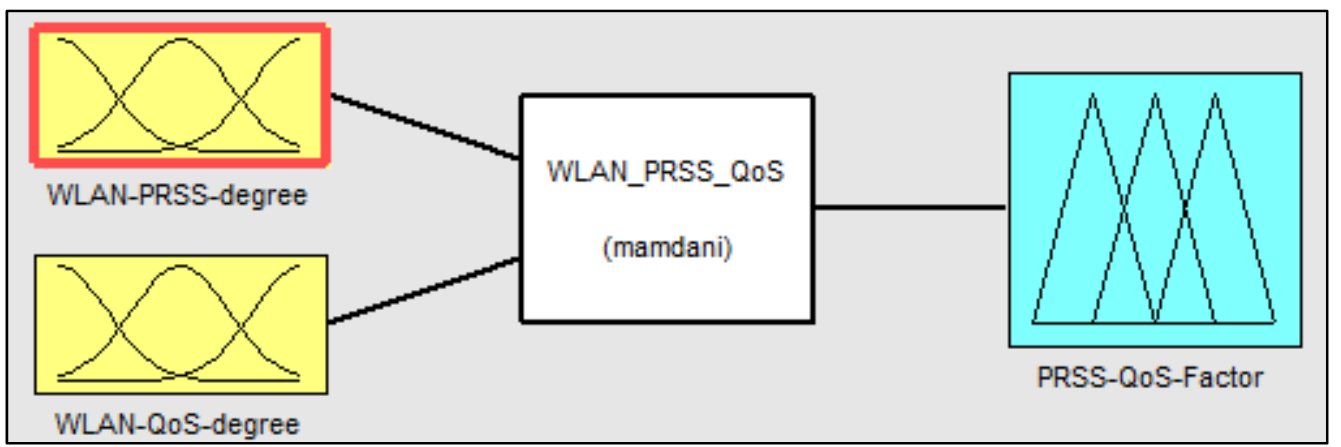

Figure 3.26: Design of FLC-3 (WLAN)

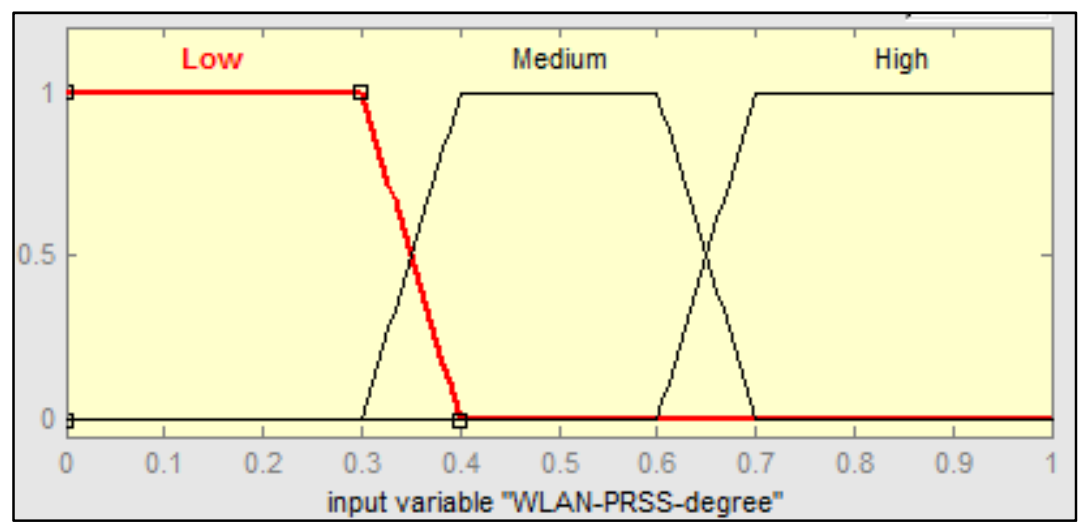

Figure 3.27: Membership Function for Input Variable PRSS of FLC-3 (WLAN)

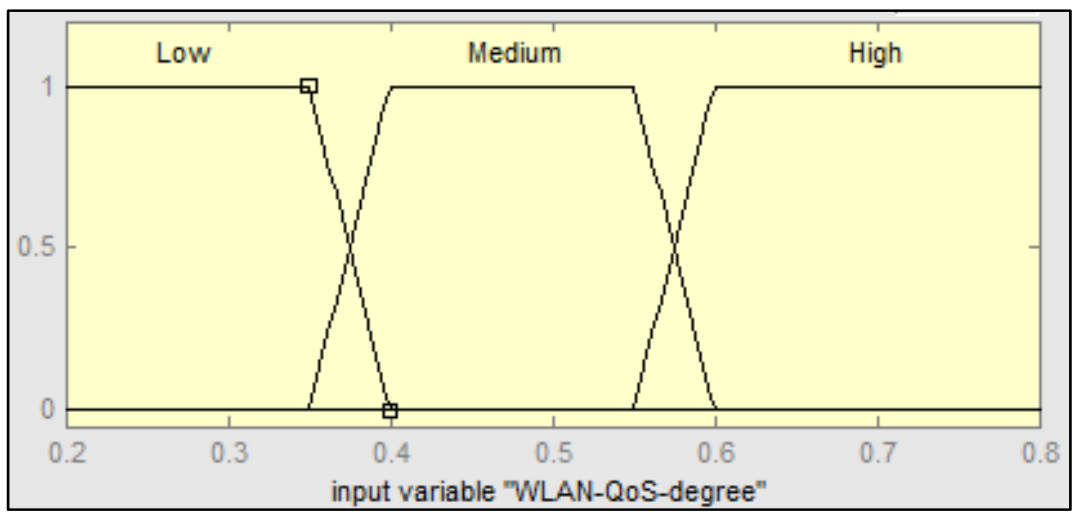

Figure 3.28: Membership Function for Input Variable QoS of FLC-3 (WLAN)

Similar FLCs are designed for WMAN, and WWAN. The only difference between these three FLCs is the different UoDs with different ranges utilized for the three network types due to the varying QoS values. 


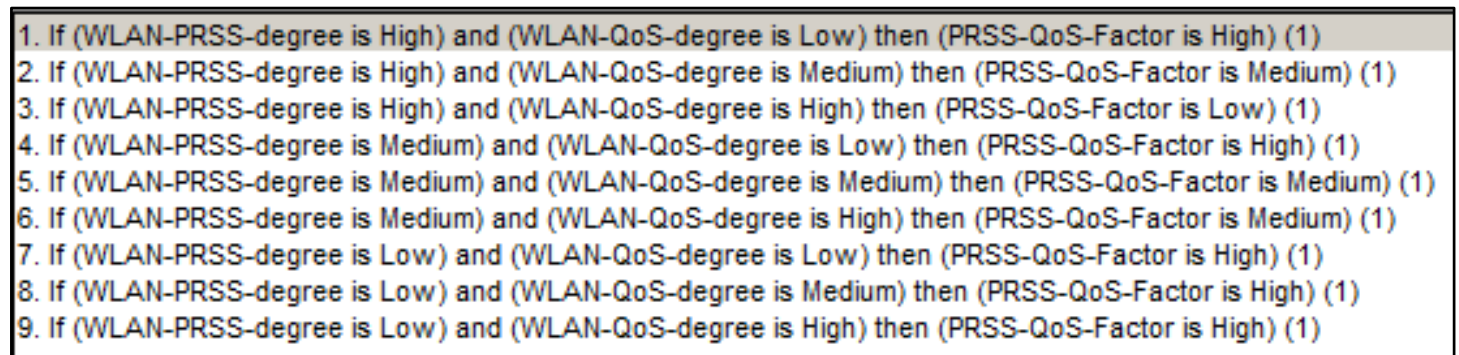

Figure 3.29: Inference Rules for FLC-3 (WLAN)

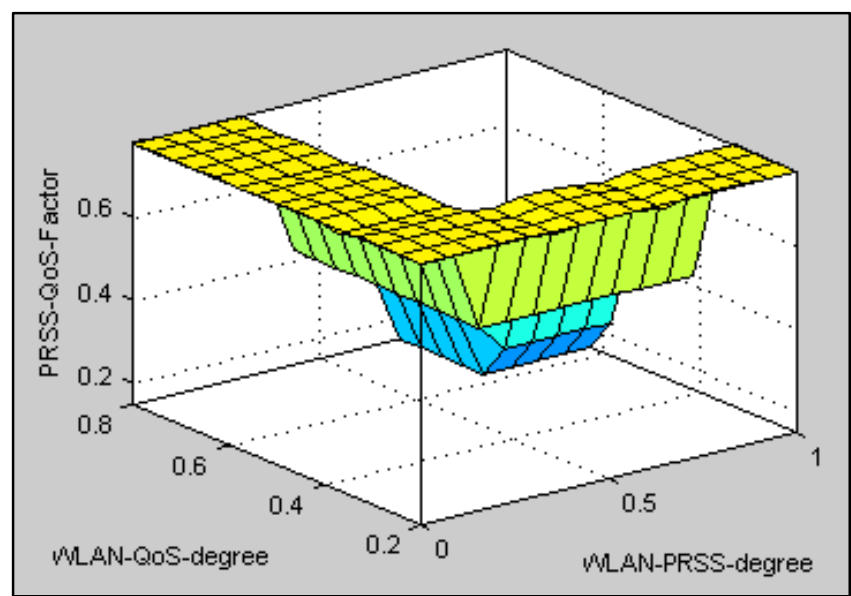

Figure 3.30: Control Surface of the Output Variable PRSS-QoS-Factor of FLC-3 (WLAN)

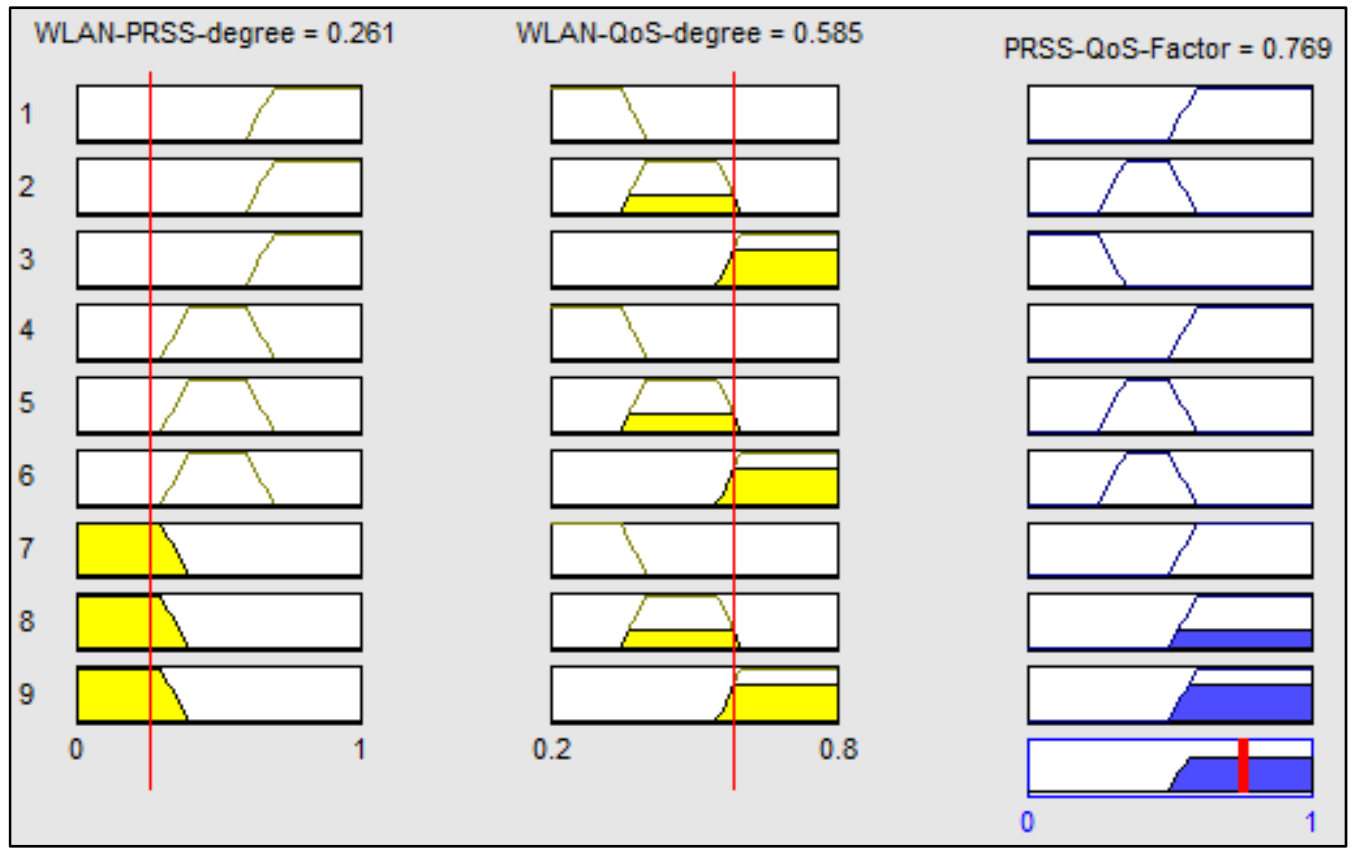

Figure 3.31: Rules Evaluation for FLC-3 (WLAN) (Low RSS and High QoS) 


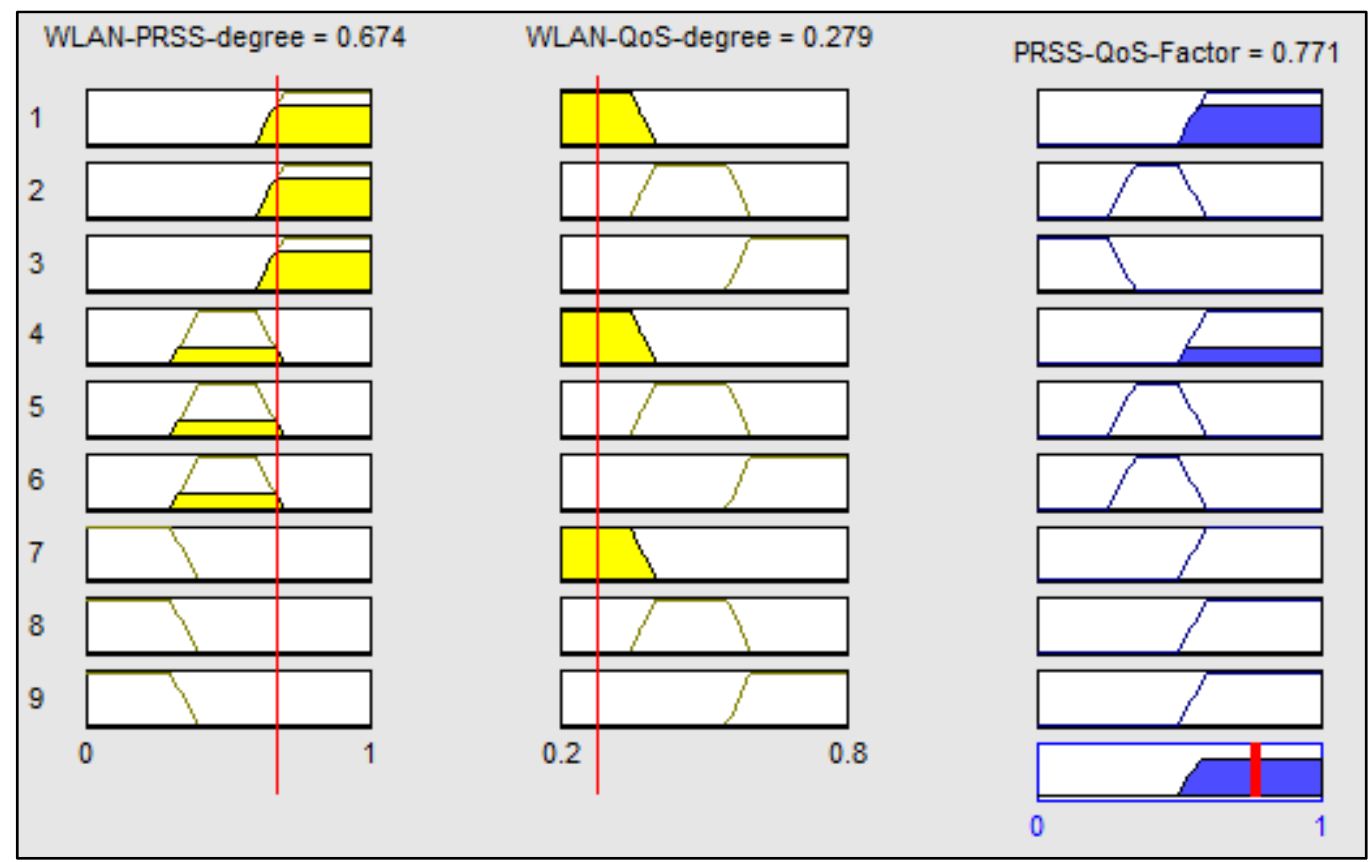

Figure 3.32: Rules Evaluation for FLC-3 (WLAN) (High RSS and Low QoS)

\subsubsection{Design of Fuzzy Logic Controller 4}

The FLC-4 is the main controller that determines the necessity of vertical handoffs based on the existing conditions of serving PoA. These conditions are evaluated using parallel FLCs to generate three different handoff factors (as discussed in the previous sections). These handoff factors serve as inputs to Mamdani based FLC-4, which in turn, outputs the VHO factor for the current PoA. Note that the PoA can be WLAN, WMAN, or WWAN. Figure 3.33 shows the design for FLC-4 where Figures 3.34-3.38 are all related to this design. A reduced number of rules (19 instead of 27) can be seen from Figure 3.35. This is due to the fact that the proposed scheme gives more importance to the RSS-QoS-Factor, as it plays a critical role in determining the quality of the currently utilized service, and maximizing the end-user satisfaction. Figures 3.39-3.40 demonstrate evaluation of these rules for two different cases, showing whether handoff is 
required or not. As can be seen from Figure 3.39, a high value of VHO-Factor is generated, indicating a high probability of handoff. This is due to the fact that the MS is currently connected to WLAN and is moving away from the AP.

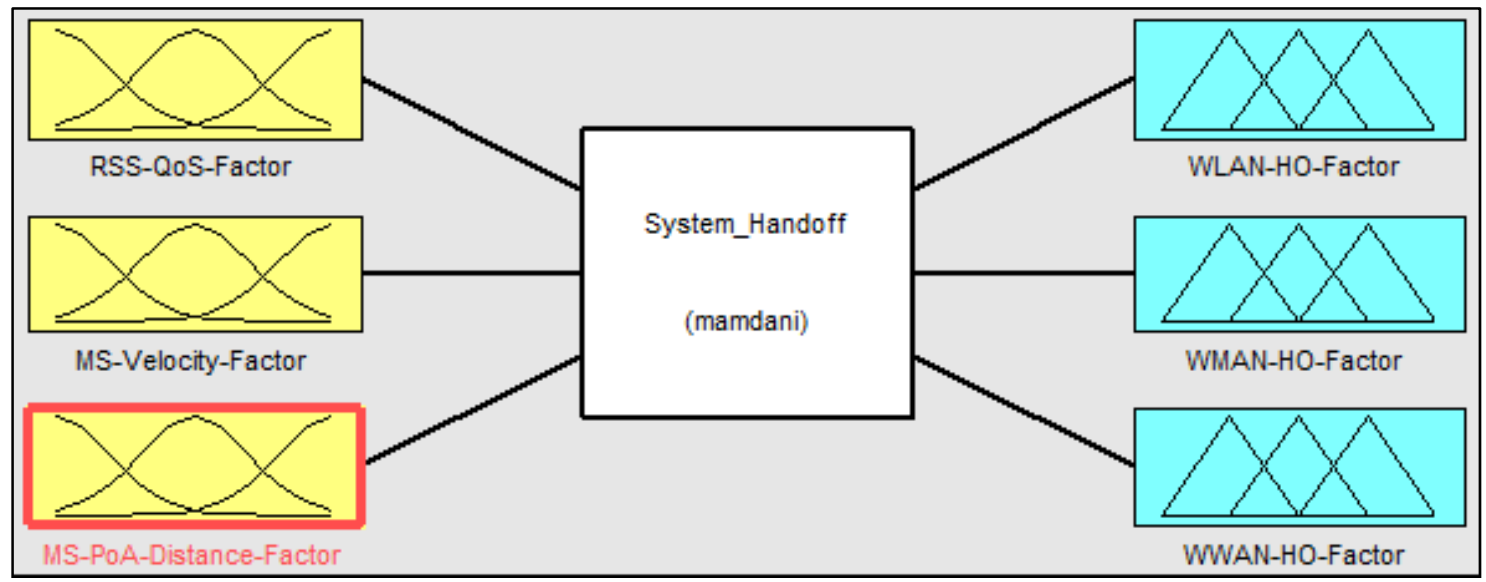

Figure 3.33: Design for FLC-4

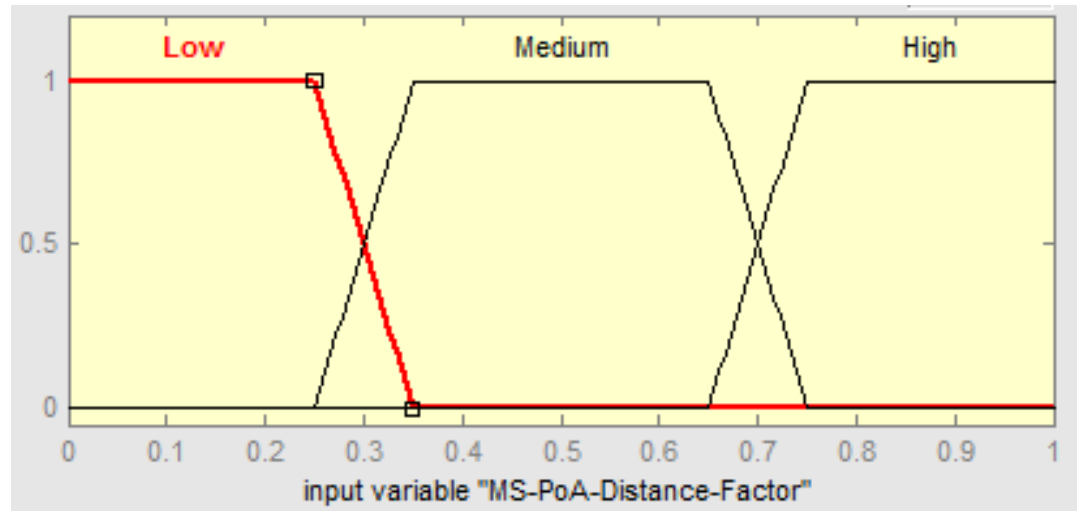

Figure 3.34: Membership function for Input Variable MS-PoA-Distance-Factor for FLC-4

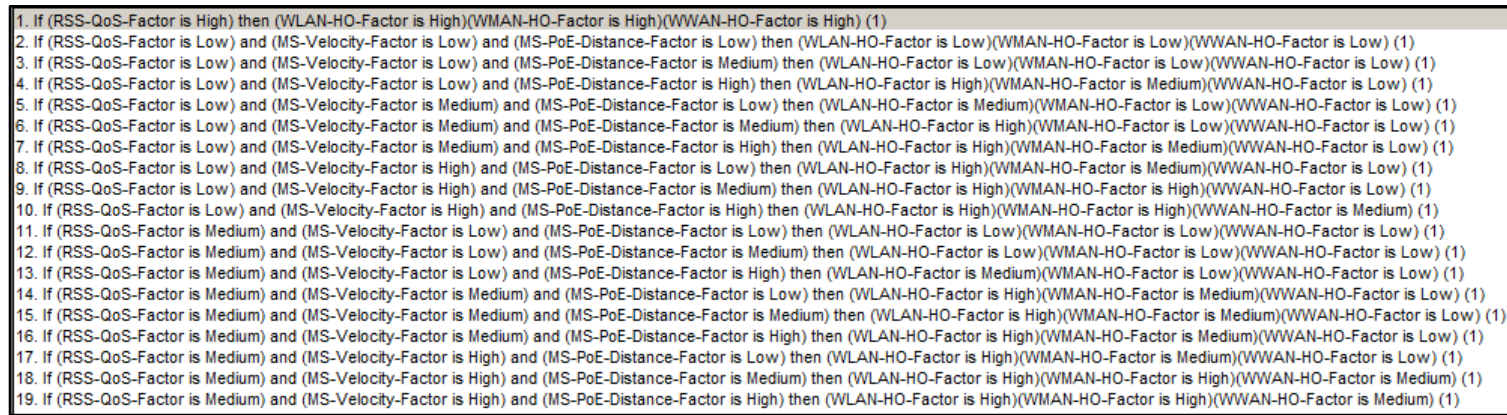

Figure 3.35: Inference Rules for FLC-4 


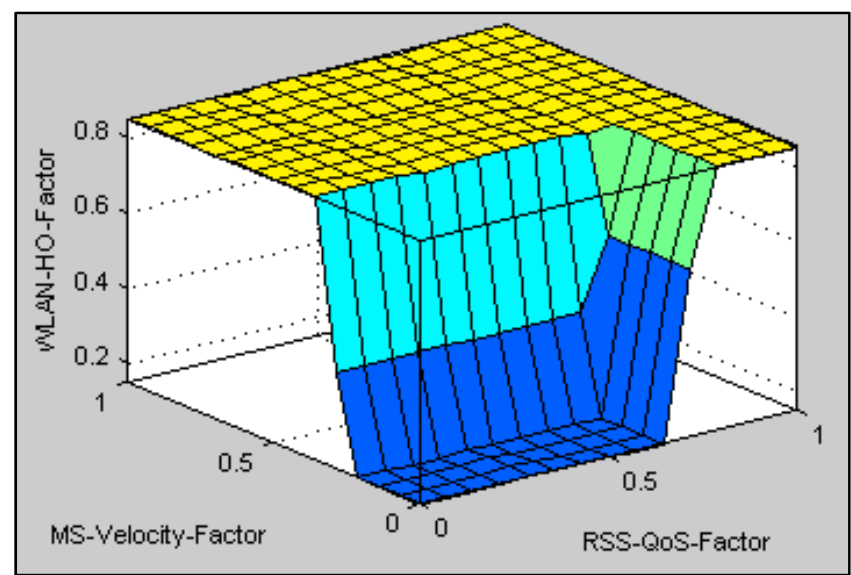

Figure 3.36: Control Surface for the Output Variable WLAN-HO-Factor for FLC-4

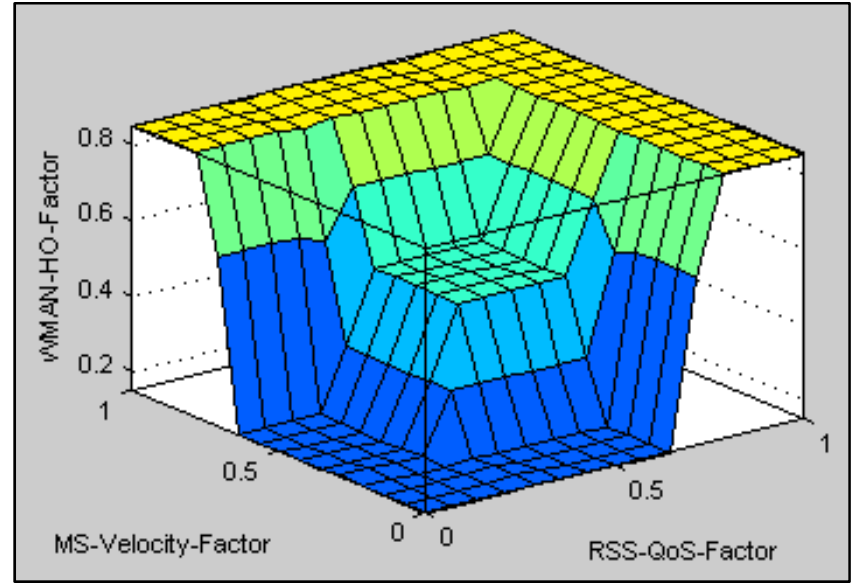

Figure 3.37: Control Surface for the Output Variable WMAN-HO-Factor for FLC-4

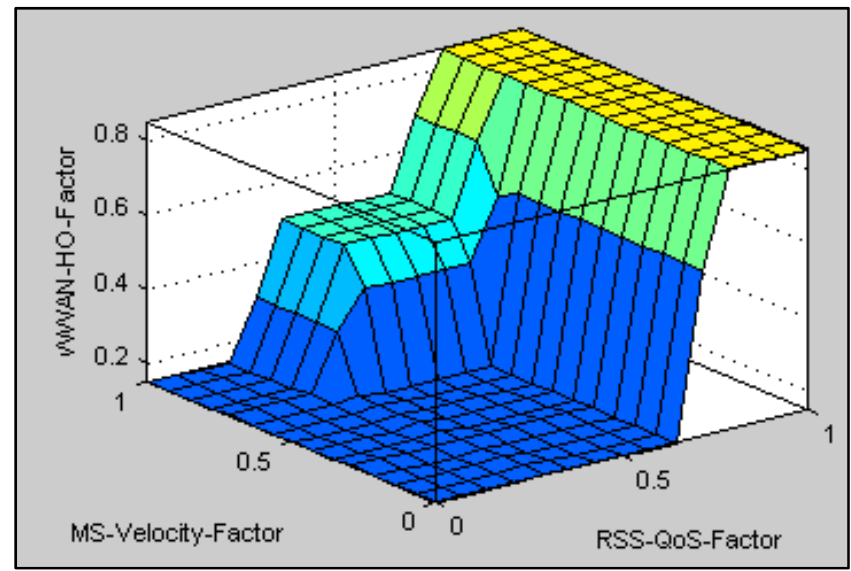

Figure 3.38: Control Surface for the Output Variable WWAN-HO-Factor for FLC-4 


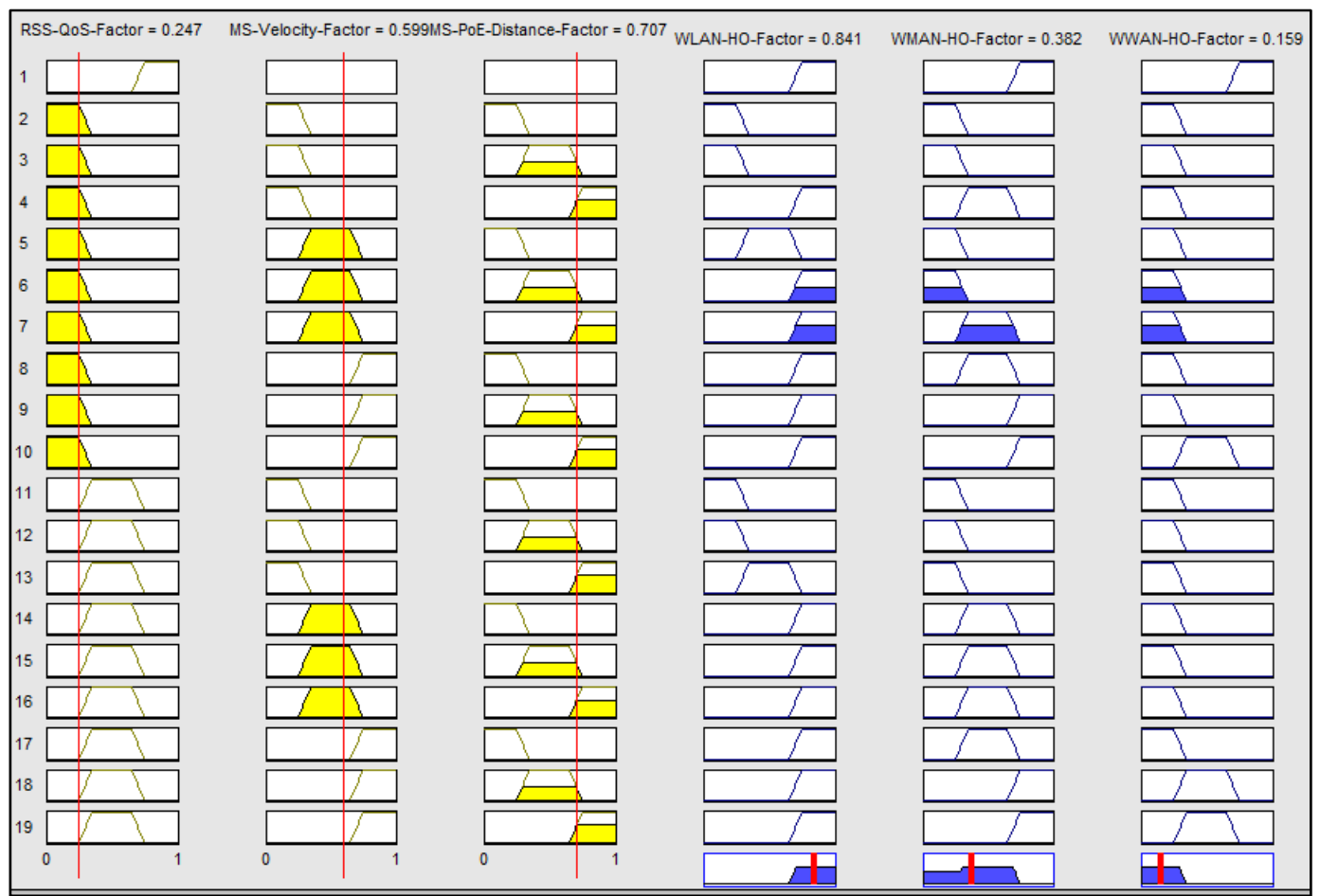

Figure 3.39: Rules Evaluation for FLC-4 (High Handoff Probability)

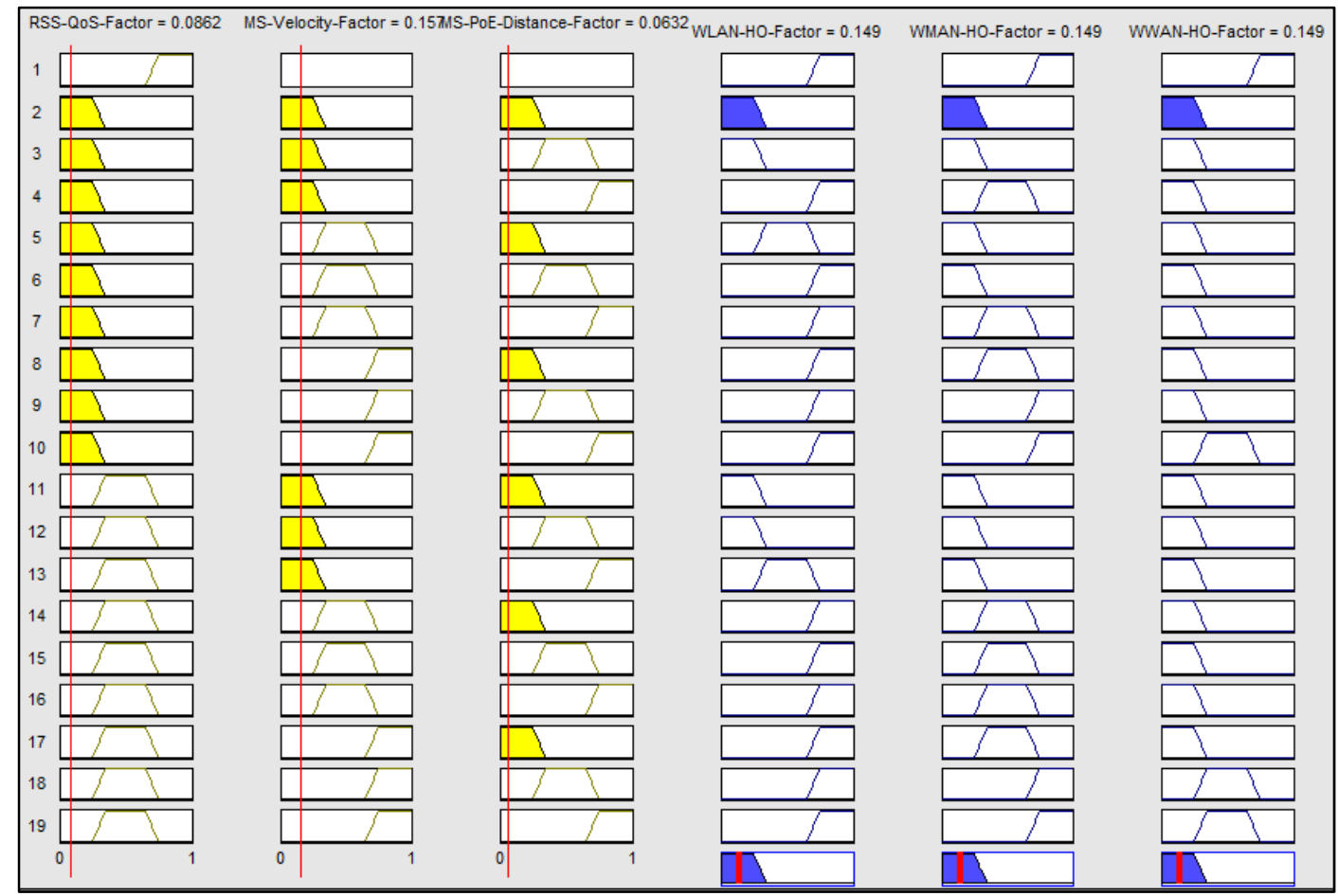

Figure 3.40: Rules Evaluation for FLC-4 (Low Handoff Probability) 


\subsubsection{HANDOFF NECESSITY ESTIMATION}

The final handoff factor that is obtained as the output from FLC-4 is compared against a threshold value to determine if a handoff from the serving PoA is required. This threshold value can be adjusted according to the sensitivity of the network types. Note that a higher value of this threshold will prevent necessary handoffs, resulting in high probability of call drops. On the other hand, a low value will result in frequent and costly handoffs, resulting in unnecessary wastage of system resources. Thus, a balanced value for this threshold is required. Performing numerous simulations and observing the need for handoffs based on different input conditions achieve this balanced value. The proposed scheme utilizes a value of 0.75 for all three network types.

\subsection{VHITS TARGET NETWORK SELECTION MODULE}

A heterogeneous wireless network comprises of different wireless access technologies including IEEE 802.11 Wi-Fi, IEEE 802.16 WiMAX, 3G/4G, and satellite network, etc. On the other hand, a variety of mobile devices, including but not limited to smartphones, iPads, etc., have surfaced during the past few years and have been evolving rapidly while becoming smarter and more powerful. Most of these mobile devices are equipped with multiple interfaces in order to obtain services via different wireless access technologies in a heterogeneous environment. These technical advancements in the area of wireless communication and technology ultimately lead towards a ubiquitous and pervasive environment offering powerful and rich connectivity; a wireless environment where multiple access technologies are available and the end-users are served with anywhere, anytime networks, the so called "Always Best Connected" (ABC) networks. 
These ABCs have been designed to provide support for different types of services that can be consumed by end-users. These rich multimedia services are characterized by different traffic demands and diverse QoS requirements that must be fulfilled in order to guarantee end-users' satisfaction.

Hence, selection of an $\mathrm{ABC}$ to meet specific application/service's QoS requirements to maximize the end-user's satisfaction is a very challenging task and involves several key attributes including RSS, QoS, traffic load, MS's velocity, offered security, and network usage cost. Since all of these key parameters play a critical role in determining an $\mathrm{ABC}$ network, ranking the candidate networks based on a single criterion will not provide an optimal result while selecting a best target network.

Several MADM approaches [126] that rank alternatives by comparing them based on relative importance of multiple criteria have been developed. Since network selection has recently become a multi-criteria problem, application of different MADM algorithms to rank candidate networks in a preferential order is considered in this research work. In the following paragraphs, different algorithms, namely TOPSIS, Fuzzy TOPSIS, and Fuzzy VIKOR are discussed. These algorithms are successfully applied to implement the target network selection mechanism of VHITS. Figure 3.41 shows an overall design of the VHITS Target Network Selection scheme. A comparison of Figures 3.2 and 3.41 shows the presence of several common components utilized by both VHITS Handoff Necessity Estimation and VHITS Target Network Selection modules. Hence, in this section the details of some of these components that are already provided in Section 3.2 are intentionally skipped and only minor exceptions are noted. 


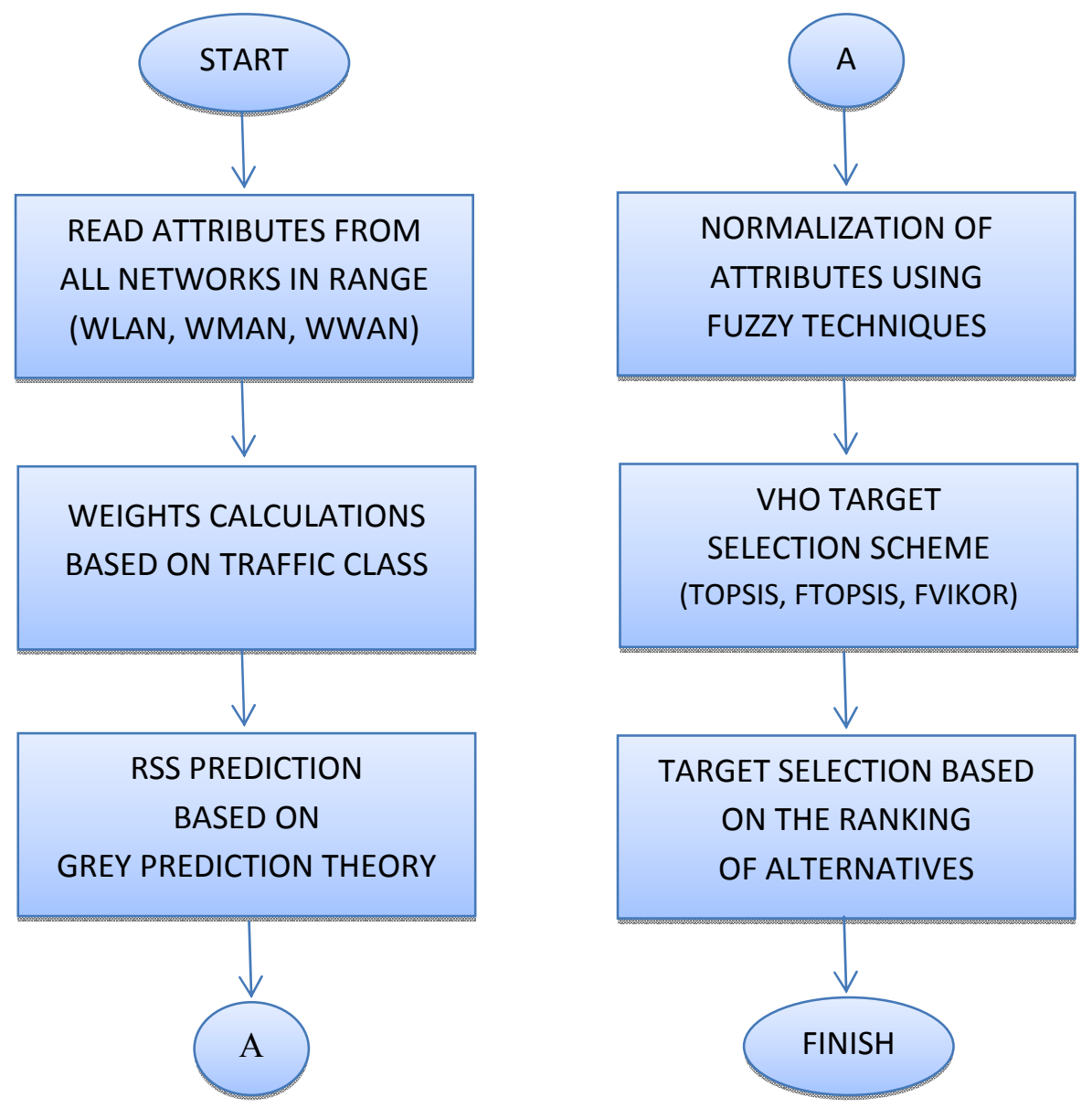

Figure 3.41: VHITS Target Network Selection Scheme

\subsubsection{SYSTEM ATTRIBUTES}

The VHITS target network selection scheme utilizes nine parameters including PRSS, individual QoS parameters (delay, jitter, PLR, and throughput), MS-velocity, traffic-load, security-preference and network-usage-cost. These nine parameters are measured from all available candidate networks that can provide coverage to the MS. As discussed in VHITS handoff necessity estimation module, we assume the availability of these system parameters to MS. 


\subsubsection{WEIGHTS CALCULATIONS FOR SYSTEM ATTRIBUTES}

Due to the fact that the effects of multiple criteria on evaluating the available alternatives have natural variances, the relative importance of each of the attributes involved in ranking the available candidate networks based on users' and/or operators' preferences is decided by assigning weights to each of the utilized attributes. Any of the methods discussed in Section 3.2.2 can be utilized to calculate these weights. The TOPSIS based network selection method can work with any of these weighting schemes as all of them generate crisp values for final weights. However, these weight calculation schemes cannot be utilized with FTOPSIS and FVIKOR ranking methods, as these fuzzy based schemes require that their attributes and corresponding weights must be either Linguistic Variables or triangular fuzzy numbers. Hence, certain modifications are needed in these weighting schemes..

\subsubsection{RSS PREDICTION USING GREY PREDICTION THEORY}

A heterogeneous wireless network is characterized by a fast fading environment where the RSS of any available candidate network can fall below a threshold that is required to maintain the connectivity with the MS. The GPT is used to predict the future RSS values for all available candidate networks. This is to ensure that the networks with strong signals are available and will remain available at the time of handoff.

\subsubsection{NORMALIZATION OF ATTRIBUTES USING FUZZY TECHNIQUES}

The crisp values of all the required attributes, from all available candidate networks, are measured and normalized using the same process as detailed in Section 3.2.5; several FISs are utilized to determine the probability of selection/rejection for an 
MS to be connected to a given network type. After this process, all system attributes with different units and ranges are normalized to a common scale, which is required by all candidate-ranking algorithms.

The TOPSIS algorithm can then be used with these normalized parameters to obtain the rank of all available networks. On the other hand, since the measured data and their corresponding membership values are crisp, it cannot be utilized directly with Fuzzy TOPSIS and Fuzzy VIKOR schemes as they rely on Linguistic Variables and/or triangular fuzzy numbers. Hence, the proposed scheme utilizes a similar scale as presented in Table 3.22 to transform these crisp values into triangular fuzzy numbers that can be utilized by these fuzzy based methods.

\subsubsection{SIGNIFICANCE OF INDIVIDUAL PARAMETERS}

The VHITS handoff necessity estimation scheme relies on the degree of QoS for a specific traffic class that is obtained based on the values of QoS parameters provided by the current PoA. This degree is calculated using a weighted sum of membership values for all QoS parameters as shown in Equation (3.32). The assignment of weights is based on the type of current service consumed by the end-user.

The VHITS target network selection scheme cannot use this degree directly since at a given instance of time, measured values of these QoS parameters from multiple candidate networks are required during the network selection process. Hence, the proposed scheme measures individual QoS parameters from each of the available candidate networks, assigns weights to these parameters, based on the traffic class currently utilized or requested by the end-user, and then feeds them to the network 
ranking algorithm. This way the VHITS target network selection algorithm can rank the candidate networks based on the strength of individualized parameters to capture their significance in the final ranking order during the network selection process.

The target selection mechanism of VHITS relies on nine different parameters obtained from all available candidate networks (alternatives). These values are arranged together to create a decision matrix. The decision matrix, along with criteria weights, is then fed into the ranking algorithms that are discussed next.

\subsection{NETWORK SELECTION (RANKING) ALGORITHMS}

This section introduces different ranking algorithms that can be utilized in the proposed scheme. As discussed previously, the users in a heterogeneous wireless environment have a need to be always best connected anywhere at anytime. In order to guarantee the continuity and the quality of the current session, vertical handoffs, based on intelligent decisions to select the optimal network, are required.

Various MADM algorithms such as SAW, MEW, ELECTRE, VIKOR, and GRA, have been proposed to make such selection decisions but application of fuzzy logic in addition to these algorithms especially in the area of wireless communication is just starting. These algorithms are used to rank the alternatives (candidate networks) according to their attractiveness (based on weighted criteria). This in turn helps to achieve the overall objective; that is to satisfy the maximum number of mobile users in a heterogeneous wireless environment.

The rest of this section discusses TOPSIS, FTOPSIS that is an extension of TOPSIS based on fuzzy logic, and Fuzzy VIKOR. To the best of our knowledge, this 
research work is the first attempt to apply FTOPSIS and Fuzzy VIKOR to the network selection problem in a heterogeneous wireless environment.

\subsubsection{NETWORK SELECTION USING TOPSIS}

TOPSIS [91, 121, 127], an MADM ranking algorithm, is designed to measure the relative efficiency of the available alternatives based on certain criteria. One of the reasons for its popularity is that it requires limited subjective inputs from decision makers, which happens to be the preference weights assigned to different criteria. The principle behind this algorithm is very simple; the chosen alternative should be as close to the ideal solution as possible and as far from the negative-ideal solution as possible. The ideal solution is a composite of the best performance values, for each attribute, exhibited by any alternative. The negative-ideal solution is the composite of the worst performance values. The distance between each alternative and these performance values is measured in the Euclidean sense to decide relative closeness to the ideal solution. Note that this distance is affected by the decision maker's subjective preferences for each criterion. The following steps are involved in TOPSIS ranking algorithm:

1. Decision Matrix Construction: A $m \times n$ decision matrix containing the ratings of each alternative w.r.t each criterion is created. This is expressed in Equation (3.37).

$$
D=\begin{gathered}
C_{1} \\
A_{1} \\
A_{2} \\
\vdots \\
A_{m}
\end{gathered}\left[\begin{array}{cccc}
d_{11} & d_{12} & \cdots & C_{n} \\
d_{21} & d_{22} & \cdots & d_{1 n} \\
\vdots & \vdots & \cdots & \vdots \\
d_{m 1} & d_{m 2} & \cdots & d_{m n}
\end{array}\right]
$$


where $A_{1}, A_{2}, \ldots, A_{m}$ are the possible alternatives, $C_{1}, C_{2}, \ldots, C_{n}$ are the criteria. Each element $d_{i j}$ of the decision matrix represents the performance rating of the alternative $A_{i}$ with respect to the criterion $C_{j}$.

2. Decision Matrix Normalization: Decision matrix is normalized based on the following equation:

$$
r_{i j}=\frac{d_{i j}}{\sqrt{\sum_{i=1}^{m} d_{i j}}} \quad i=1,2, \ldots, m \quad j=1,2, \ldots, n
$$

where $r_{i j}$ is the normalized value of element $d_{i j}$.

3. Weighted Normalized Decision Matrix Construction: This matrix is constructed by multiplying each element $r_{i j}$ with its associated weight $w_{j}$, as follows:

$$
v_{i j}=r_{i j} \times w_{j}
$$

4. Calculation of Positive \& Negative Ideal Solution: The positive and negative ideal solutions, $A^{+}$and $A^{-}$, respectively, are defined as:

$A^{+}=\left(v_{1}^{+}, v_{2}^{+}, \ldots, v_{n}^{+}\right)=\left\{\left(\max _{i} v_{i j} \mid j \in C_{B}\right),\left(\min _{i} v_{i j} \mid j \in C_{C}\right)\right\}$

$A^{-}=\left(v_{1}^{-}, v_{2}^{-}, \ldots, v_{n}^{-}\right)=\left\{\left(\min _{i} v_{i j} \mid j \in C_{B}\right),\left(\max _{i} v_{i j} \mid j \in C_{C}\right)\right\}$

where $C_{B}$ and $C_{C}$ denote the sets with benefit and cost criteria, respectively.

5. Calculation of Separation between Alternatives \& Ideal Solutions: The separation (distance) between each alternative, from the positive ideal $\left(S_{i}^{+}\right)$and negative ideal solutions $\left(S_{i}^{-}\right)$, are calculated as follows:

$S_{i}^{+}=\sqrt{\left(v_{i j}-v_{i}^{+}\right)^{2}} \quad i=1,2, \ldots, m \quad j=1,2, \ldots, n$ 


$$
S_{i}^{-}=\sqrt{\left(v_{i j}-v_{i}^{-}\right)^{2}} \quad i=1,2, \ldots, m \quad j=1,2, \ldots, n
$$

6. Calculation of Relative Closeness to the Ideal Solution: This step involves calculating the relative closeness to the ideal solution, which is defined as:

$$
C_{i}=\frac{S_{i}^{-}}{S_{i}^{-}+S_{i}^{+}} \quad i=1,2, \ldots, m
$$

7. Ranking of the Alternatives: Sorting the values of relative closeness $C_{i}$ in descending order, allows the ranking of the alternative. The best alternative has the highest value of $C_{i}$.

As part of the VHITS target network selection scheme, MATLAB code is implemented to perform the selection of the best network among the other available candidates, using a modified version of the above mentioned TOPSIS algorithm. The proposed scheme modifies the original TOPSIS algorithm by skipping the decision matrix normalization step (step 2). This is due to the fact that the proposed scheme normalizes all the parameters for all available candidate networks in the range $[0,1]$, using fuzzy techniques. This is previously discussed in detail in Sections 3.2.5 and 3.3.4. The preference weights that are required by the TOPSIS algorithm are obtained by using different techniques highlighted in Section 3.2.2. Numerical examples illustrating the VHITS target network selection mechanism are provided in Chapter 4.

\subsubsection{NETWORK SELECTION USING FUZZY TOPSIS}

A wireless environment is characterized by its dynamic nature, inherent uncertainty, and imprecise parameters and constraints. Network parameters like throughput, RSS, and network delays, etc., are intrinsically imprecise. Due to this 
vagueness, the accurate measurement of these network parameters in a wireless environment is a difficult task.

In the past, several MADM algorithms are used to establish rankings among available candidate networks, but due to the imprecise and vague nature of the input data, they are unable to produce efficient handoff decisions; the uncertainty in user preferences (in the form of criteria weights) are considered while the impreciseness in the measured data is ignored.

In the classical TOPSIS method, the ratings of the alternatives and the weights of the criteria are known precisely and crisp values are assumed and used during the ranking process. Therefore, an extension of TOPSIS method [128, 129] is proposed that deals with fuzzy data, where the weights of the attributes and the performance ratings of all available alternatives are evaluated using Linguistic Variables. These Linguistic Variables can be expressed as trapezoidal or triangular fuzzy numbers. In this research work, Linguistic Variables and triangular fuzzy numbers, defined in Table 3.22, are utilized. The details of the FTOPSIS algorithm are provided as follows:

1. Formation of Committee of Decision-Makers: A committee of $k$ decisionmakers is formed, where fuzzy ratings of alternatives and weights of criteria obtained from each decision maker $D_{k}$ can be represented in terms of a triangular fuzzy number $\tilde{x}=(l, m, u)$ whose membership function is given in Equation (3.12).

2. Fuzzy Decision Matrix Construction: This step is the same as the classical TOPSIS, with the exception that the ratings for all attributes are 
represented as triangular fuzzy numbers instead of crisp values. This is shown as

$$
\widetilde{D}_{k}=\begin{gathered}
C_{1} \\
A_{1} \\
A_{2} \\
\vdots \\
A_{m}
\end{gathered}\left[\begin{array}{cccc}
\tilde{d}_{11} & \tilde{d}_{12} & \cdots & C_{n} \\
\tilde{d}_{21} & \tilde{d}_{22} & \cdots & \tilde{d}_{1 n} \\
\vdots & \vdots & \cdots & \vdots \\
\tilde{d}_{m 1} & \tilde{d}_{m 2} & \cdots & \tilde{d}_{m n}
\end{array}\right]
$$

where element $\tilde{d}_{i j}$ of the fuzzy decision matrix $\widetilde{D}_{k}$ is the fuzzy performance rating of the alternative $A_{i}$ with respect to the criterion $C_{j}$, provided by the $k^{\text {th }}$ decision maker.

3. Aggregation of Ratings and Weights from k Decision Makers: The fuzzy ratings for alternatives and fuzzy weights for each attributes obtained from $k$ decision makers are aggregated and given as:

$\widetilde{D}=\left(d_{l}, d_{m}, d_{u}\right) \quad \widetilde{W}_{j}=\left(w_{j l}, w_{j m}, w_{j u}\right) \quad k=1,2, \ldots, K$

where $d_{l}=\min _{k}\left(d_{l}^{k}\right), \quad d_{m}=\frac{1}{K} \sum_{k=1}^{K} d_{m}^{k}, \quad d_{u}=\max _{k}\left(d_{u}^{k}\right)$

$d_{i j l}=\min _{k}\left(d_{i j l}^{k}\right), \quad d_{i j m}=\frac{1}{K} \sum_{k=1}^{K} d_{i j m}^{k}, \quad d_{i j u}=\max _{k}\left(d_{i j u}^{k}\right)$

$w_{j l}=\min _{k}\left(w_{j l}^{k}\right), \quad w_{j m}=\frac{1}{K} \sum_{k=1}^{K} w_{j m}^{k}, \quad w_{j u}=\max _{k}\left(w_{j u}^{k}\right)$

where $d_{i j l}$, and $d_{i j u}$ are the lower and upper bounds of matrix element $d_{i j}$, respectively, represented as a triangular fuzzy number. The lower and upper bounds of triangular fuzzy number representing the weight of the $j^{\text {th }}$ attribute is denoted by $w_{j l}$, and $w_{j u}$, respectively.

4. Fuzzy Decision Matrix Normalization: Normalization may or may not be necessary depending upon the Linguistic Variables and their 
corresponding triangular fuzzy numbers. In most cases, the fuzzy decision matrix is already normalized since the triangular fuzzy numbers belongs to the range $[0,1]$. Then the element $\tilde{r}_{i j}=\left(\tilde{r}_{i j l}, \tilde{r}_{i j m}, \tilde{r}_{i j u}\right)$ represents the triangular fuzzy number of the normalized value for alternative $i$ with respect to attribute $j$. In case the normalization is necessary, a linear scale transformation can be used as follows:

$$
\tilde{r}_{i j}=\left(\frac{\tilde{r}_{i j l}}{b_{j}^{+}}, \frac{\tilde{r}_{i j m}}{b_{j}^{+}}, \frac{\tilde{r}_{i j u}}{b_{j}^{+}}\right) \quad b_{j}^{+}=\max _{i} \tilde{r}_{i j u} \quad j \in B
$$

or

$$
\tilde{r}_{i j}=\left(\frac{c_{j}^{-}}{\tilde{r}_{i j u}}, \frac{c_{j}^{-}}{\tilde{r}_{i j m}}, \frac{c_{j}^{-}}{\tilde{r}_{i j l}}\right) \quad c_{j}^{-}=\min _{i} \tilde{r}_{i j l} \quad j \in C
$$

where $B$ and $C$ are the sets of Benefit and Cost based criteria, respectively.

5. Weighted Normalized Decision Matrix Construction: This matrix is constructed by multiplying each element $\tilde{r}_{i j}$ by its associated weight $\widetilde{w}_{j}$, as follows:

$$
\tilde{v}_{i j}=\tilde{r}_{i j} \times \widetilde{w}_{j}
$$

6. Calculation of Fuzzy Positive \& Negative Ideal Solutions: The fuzzy positive and negative ideal solutions, $\tilde{A}^{+}$(FPIS) and $\tilde{A}^{-}$(FNIS), respectively, are defined as:

$$
\begin{array}{ll}
\tilde{A}^{+}=\left(\tilde{v}_{1}^{+}, \tilde{v}_{2}^{+}, \ldots, \tilde{v}_{n}^{+}\right) & \tilde{v}_{j}^{+}=\max _{i} v_{i j u} \\
\tilde{A}^{-}=\left(v_{1}^{-}, v_{2}^{-}, \ldots, v_{n}^{-}\right) & \tilde{v}_{j}^{-}=\min _{i} v_{i j l}
\end{array}
$$


where $\tilde{v}_{j}^{+}$, and $\tilde{v}_{j}^{-}$represent the maximum and minimum ratings of the alternative with respect to the $j^{\text {th }}$ criterion, respectively, and $v_{i j u}$ and $v_{i j l}$ represent the upper and lower bound of the triangular fuzzy number $\tilde{v}_{i j}$.

7. Calculation of Separation between Alternatives \& Fuzzy Ideal Solutions: The separation (distance) between each alternative from the fuzzy positive ideal and fuzzy negative ideal solutions are calculated as follows:

$$
\begin{aligned}
& d_{i}^{+}=\sum_{j=1}^{n} d_{v}\left(\tilde{v}_{i j}, \tilde{v}_{j}^{+}\right) \quad i=1,2, \ldots, m \\
& d_{i}^{-}=\sum_{j=1}^{n} d_{v}\left(\tilde{v}_{i j}, \tilde{v}_{j}^{-}\right) \quad i=1,2, \ldots, m
\end{aligned}
$$

where $d_{v}(*, *)$ is the distance measurement between two fuzzy numbers calculated by using the vertex method as follows:

$$
d_{v}(\tilde{A}, \tilde{B})=\sqrt{\frac{1}{3}\left[\left(a_{l}-b_{l}\right)^{2}+\left(a_{m}-b_{m}\right)^{2}+\left(a_{u}-b_{u}\right)^{2}\right]}
$$

8. Calculation of Relative Closeness to the Ideal Solution: This step involves calculating the relative closeness to the fuzzy ideal solutions, which is defined as:

$$
C_{i}=\frac{d_{i}^{-}}{d_{i}^{-}+d_{i}^{+}} \quad i=1,2, \ldots, m
$$

9. Ranking of the Alternatives: Sorting the calculated values of relative closeness $C_{i}$ in descending order, allows for the ranking of the alternatives. The best alternative has the highest value of $C_{i}$, where alternative $A_{i}$ will be closer to $\tilde{A}^{+}$and farther from $\tilde{A}^{-}$as $C_{i}$ approaches 1 .

A FTOPSIS module with minor modifications is implemented in MATLAB to support the VHITS target network selection scheme. As explained previously, the 
original FTOPSIS algorithm requires the usage of Linguistic Variables for rating the alternatives and the weights of the attributes. Since the VHITS target network selection scheme relies on crisp measurements obtained from all available networks, a modification is done in the original algorithm to transform these crisp values into triangular fuzzy numbers for each measured attributes from all available candidate networks. The process explained in Section 3.3.4 is followed to transform and normalize these crisp values into triangular fuzzy numbers. These TFNs are then used to form the fuzzy decision matrix that is required by the FTOPSIS algorithm. It is worth mentioning that the algorithm is implemented in a flexible way, such that it can utilize both Linguistic Variables and TFNs to form the fuzzy decision matrix.

The first three steps in the original FTOPSIS algorithm collect information from multiple decision makers and create an aggregated fuzzy decision matrix for ratings and an aggregated preference weights vector for all attributes. In the network selection problem, using FTOPSIS, these steps do not fit well, as the information is measured directly from the available networks and not provided by any decision makers; the only exception to this is the weight vector, where the weights for some of the attributes such as RSS and QoS can be collected and aggregated from multiple design engineers and can be pre-assigned. Hence, this research work proposes two alternatives:

- Skip these three initial steps and form the fuzzy decision matrix from the attributes values measured directly from all available networks.

- Read multiple samples of each attributes from all available networks and then, after aggregating these samples create the fuzzy decision matrix, following the steps outlined in the original FTOPSIS algorithm. This is the 
recommended approach as it will incorporate the fuzziness and vagueness of imprecise values of each measured attributes.

The implemented FTOPSIS algorithm is capable of utilizing both alternatives.

The preference weights for the attributes required by the FTOPSIS algorithm must be calculated using Linguistic Variables. Hence, the weighting methods proposed in Section 3.2.2 cannot be used directly with FTOPSIS as the final weights generated by these schemes are crisp in nature. This can be seen in Table 3.32, where a comparison between different weighting schemes is shown. Hence two alternatives, for calculating weights for FTOPSIS algorithm, are proposed in this research work:

- The direct use of Linguistic Variables for all the attributes: These weights in terms of Linguistic Variables can be obtained from multiple decision makers that can include network operators as well as the end-users. These preferences from operators and end-users can be aggregated following the first three steps defined in the original FTOPSIS algorithm.

- The usage of Linguistic Variables for all the attributes, in addition to performing a similar weight elicitation technique, as proposed in Section 3.2.2.1: The benefit is two folds; the resolution of interdependence between any two attributes at the same level of hierarchy and the effective handling of intrinsic imprecision and vagueness associated with enduser's preferences by utilizing triangular fuzzy numbers.

The FTOPSIS algorithm that is implemented as part of this research can utilize both alternatives to calculate the final weights for each attributes. A separate weight elicitation module is implemented to support weight calculations based on the second 
alternative. Since this involves several mathematical and matrix operations on triangular fuzzy numbers, MATLAB modules are created to support fuzzy operations on triangular fuzzy numbers. This is another contribution of this research work. Equations (3.58) to (3.71) show mathematical operations [130] on triangular fuzzy numbers that are utilized by this research work.

$$
\text { Assuming } \tilde{x}=\left(x_{l}, x_{m}, x_{u}\right) \text { and } \widetilde{y}=\left(y_{l}, y_{m}, y_{u}\right) \text { are two triangular fuzzy numbers, }
$$
then,

$$
\begin{array}{ll}
\tilde{x}(+) \tilde{y}=\left(x_{l}+y_{l}, x_{m}+y_{m}, x_{u}+y_{u}\right) & \\
\tilde{x}(-) \tilde{y}=\left(x_{l}-y_{u}, x_{m}-y_{m}, x_{u}-y_{l}\right) & \\
\tilde{x}(\times) \tilde{y}=\left(x_{l} \times y_{l}, x_{m} \times y_{m}, x_{u} \times y_{u}\right) & \tilde{x}>0, \quad \tilde{y}>0 \\
\tilde{x}(\times) \tilde{y}=\left(x_{l} \times y_{u}, x_{m} \times y_{m}, x_{u} \times y_{l}\right) & \tilde{x}<0, \quad \tilde{y}>0 \\
\tilde{x}(\times) \tilde{y}=\left(x_{u} \times y_{u}, x_{m} \times y_{m}, x_{l} \times y_{l}\right) & \tilde{x}<0, \quad \tilde{y}<0 \\
\tilde{x}(\div) \tilde{y}=\left(\frac{x_{l}}{y_{u}}, \frac{x_{m}}{y_{m}}, \frac{x_{u}}{y_{l}}\right) & \tilde{x}>0, \quad \tilde{y}>0 \\
\tilde{x}(\div) \tilde{y}=\left(\frac{x_{u}}{y_{u}}, \frac{x_{m}}{y_{m}}, \frac{x_{l}}{y_{l}}\right) & \quad \beta \in \mathcal{R}, \quad \beta>0 \\
\tilde{x}(\div) \tilde{y}=\left(\frac{x_{u}}{y_{l}}, \frac{x_{m}}{y_{m}}, \frac{x_{l}}{y_{u}}\right) & \\
\beta . \tilde{x}=\left(\beta \cdot x_{l}, \beta \cdot x_{m}, \beta \cdot x_{u}\right) & \\
\beta . \tilde{x}=\left(\beta \cdot x_{u}, \beta \cdot x_{m}, \beta \cdot x_{l}\right) & \\
\frac{1}{\tilde{x}}=\left(\frac{1}{x_{u}}, \frac{1}{x_{m}}, \frac{1}{x_{l}}\right) & \\
(-\tilde{x})=\left(-x_{u},-x_{m},-x_{l}\right) & \\
\max (\tilde{x}, \tilde{y})=\left\{\max \left(x_{l}, y_{l}\right), \max \left(x_{m}, y_{m}\right), \max \left(x_{u}, y_{u}\right)\right\} \\
\min (\tilde{x}, \tilde{y})=\left\{\min \left(x_{l}, y_{l}\right), \min \left(x_{m}, y_{m}\right), \min \left(x_{u}, y_{u}\right)\right\}
\end{array}
$$


A fuzzy number $\tilde{A}$ is called positive fuzzy number if $\mu_{\tilde{A}}(x)=0, \forall x<0$.

\subsubsection{NETWORK SELECTION USING FUZZY VIKOR}

VIKOR is an MADM method that is developed to optimize the multi-attribute based complex systems. It is a compromised programming approach based on an aggregating function that represents closeness to the ideal solution. VIKOR [81] is able to determine a compromise-ranking list of alternatives in the presence of conflicting criteria. This characteristic makes VIKOR an appropriate ranking and decision algorithm for handoff decisions in heterogeneous wireless networks.

Fuzzy VIKOR $[80,131,132]$ is an extension to the original algorithm including the domain of vagueness and fuzziness. The steps of Fuzzy VIKOR are outlined as follows:

1. Aggregation of Decision Makers' ratings and weights: The initial step, to construct a group of decision makers and identify the appropriate Linguistic Variables to evaluate the rating for alternatives and weight of criteria, is the same, as presented in FTOPSIS. The aggregation of these ratings and weights from $k$ decision makers is obtained by:

$$
\begin{gathered}
\widetilde{w}_{j}=\frac{1}{k}\left[\widetilde{w}_{j}^{1}+\widetilde{w}_{j}^{2}+\cdots+\widetilde{w}_{j}^{k}\right] \\
\tilde{x}_{i j}=\frac{1}{k}\left[\tilde{x}_{i j}^{1}+\tilde{x}_{i j}^{2}+\cdots+\tilde{x}_{i j}^{k}\right]
\end{gathered}
$$

where $\widetilde{w}_{j}$ is the aggregated weight of the $j^{\text {th }}$ attribute and $\tilde{x}_{i j}$ is the aggregated rating of the $i^{\text {th }}$ alternative with respect to the $j^{\text {th }}$ attribute in the fuzzy decision matrix. 
2. Determination of the Fuzzy Best and Fuzzy Worst Values: The fuzzy best value (FBV) $\tilde{f}_{j}^{+}$and the fuzzy worst value (FWV) $\tilde{f}_{j}^{-}$for all criteria is determined by:

$$
\begin{aligned}
& \tilde{f}_{j}^{+}=\max _{i} \tilde{x}_{i j} \quad j \in \text { Benefit } \\
& \tilde{f}_{j}^{-}=\min _{i} \tilde{x}_{i j} \quad j \in \text { Cost }
\end{aligned}
$$

3. Computation of Separation Measures: The separation measure $\tilde{S}_{i}$ of alternative $A_{i}$ from the FBV, and the separation measure $\widetilde{R}_{i}$ from the FWV are defined as:

$$
\begin{aligned}
& \tilde{S}_{i}=\sum_{j=1}^{n} w_{j} \frac{\tilde{f}_{j}^{+}-\tilde{x}_{i j}}{\tilde{f}_{j}^{+}-\tilde{f}_{j}^{-}} \\
& \tilde{R}_{i}=\max _{j}\left[w_{j} \frac{\tilde{f}_{j}^{+}-\tilde{x}_{i j}}{\tilde{f}_{j}^{+}-\tilde{f}_{j}^{-}}\right]
\end{aligned}
$$

4. Computation of indices $\tilde{S}^{+}, \tilde{S}^{-}, \tilde{R}^{+}, \tilde{R}^{-}$, and $\tilde{Q}_{i}$ : These indices are calculated as follows:

$$
\tilde{Q}_{i}=v\left[\begin{array}{c}
\tilde{S}_{i}-\tilde{S}_{i}^{+} \\
\tilde{S}_{i}^{-}-\tilde{S}_{i}^{+}
\end{array}\right]+(1-v)\left[\frac{\tilde{R}_{i}-\tilde{R}_{i}^{+}}{\tilde{R}_{i}^{-}-\tilde{R}_{i}^{+}}\right]
$$

where $\tilde{S}^{+}=\min _{i} \tilde{S}_{i}$ defines the index with a maximum majority rule, $\tilde{R}^{+}=\min _{i} \tilde{R}_{i}$ defines the index with a minimum individual regret of opponent, $\tilde{S}^{-}=\max _{i} \tilde{S}_{i}, \tilde{R}^{-}=\max _{i} \tilde{R}_{i}$, and $v$ is the weight in the strategy of the maximum group utility (or the majority of the criteria), usually $v=0.5$.

5. Defuzzification of Triangular Fuzzy Numbers: In the original algorithm, triangular fuzzy numbers are converted into crisp values using Chen's [133] method of maximizing set and minimizing set. In order to simplify 
the process, this research work utilizes the centroid method to perform defuzzification as follows:

$$
\operatorname{Crisp}_{\tilde{A}}=\frac{1}{6}\left(l_{\tilde{A}}+4 m_{\tilde{A}}+u_{\tilde{A}}\right)
$$

6. Ranking the Alternatives: The ranking of the alternatives is based on the crisp values of $\widetilde{Q}_{i}$, as this index implies the separation measure of the alternative $A_{i}$ from the best alternative. This means an alternative with better performance, as compared with others, is indicated by the smaller value of $\tilde{Q}_{i}$.

7. Propose Compromise Solution: The last step of the process is to propose compromise solution $A^{\prime}$, using the crisp values of the $\tilde{Q}_{i}$ index based on the following condition:

- Acceptable Advantage Condition: This is given by:

$$
\left(Q_{A^{\prime \prime}}-Q_{A^{\prime}}\right) \geq D Q
$$

where $D Q=\frac{1}{M-1}, M$ is the number of available alternatives, and $A^{\prime \prime}$ is the alternative that comes out in second position based on the minimum values of $Q$ index.

If the above condition is not satisfied, then $A^{\prime}, A^{\prime \prime}, \ldots, A^{(m)}$ are, compromised candidates and the alternatives are ranked based on the ascending values of the $Q$ index.

The ranking scheme based on Fuzzy VIKOR is implemented in MATLAB as part of our VHITS target network selection scheme. Like FTOPSIS, this scheme cannot utilize the different weight elicitation techniques discussed earlier. The calculations of the 
priority weights are based on the same module implemented for the FTOPSIS ranking algorithm. Once again, the implemented scheme can accept Linguistic Variables or triangular fuzzy numbers as inputs. To the best of our knowledge, this research work is the first attempt to apply fuzzy VIKOR to select a best network among other available candidate networks in a heterogeneous wireless environment. 


\section{CHAPTER 4}

\section{SIMULATIONS AND RESULTS}

In this chapter, the performance evaluations of the proposed scheme are presented. The two developed modules, VHITS handoff necessity estimation and VHITS target network selection, are simulated and evaluated using a test-bed that is developed based on the concepts of RUNE. Four types of traffic classes, Conversational, Streaming, Background, and Interactive, using three types of wireless networks, WLAN, WMAN, and WWAN, are utilized in evaluating the performance of the proposed scheme. Simulations and comparisons are also carried out among the different types of preference weighting schemes and network selection \& ranking algorithms.

This chapter is organized into six major sections. Section 4.1 presents numerical examples based on different scenarios to verify the validity and usability of the proposed model. Section 4.2 provides the details of the implemented test-bed to simulate the proposed scheme including the mobility, propagation, and traffic models implemented using RUNE. Simulation results assuming a single mobile station in a heterogeneous wireless network are presented in Section 4.3. In Section 4.4, simulations are performed strictly based on user preferences that are set manually. Section 4.5 presents results considering a multi-user heterogeneous wireless environment. Finally, Section 4.6 concludes the chapter with a brief comparison of different selection schemes.

\subsection{NUMERICAL EXAMPLES}

In this section, numerical examples, using a scenario-based approach, are provided to verify and validate the usability of different aspects of VHITS. 
Table 4.1: Different Parameters for Current PoA

\begin{tabular}{|l|c|}
\multicolumn{1}{|c|}{ Parameters } & Values \\
\hline Current PoA & WLAN \\
\hline Current Traffic Class & Streaming \\
\hline Metric Weight Scheme & AHP \\
\hline MS-Velocity (m/s) & 0 (Low) \\
\hline MS-PoA Distance (m) & 10 (Near) \\
\hline RSS Samples (dbm) & $-58.5,-55.3,-57.6,-59.8$ \\
\hline PRSS using GPT (dbm) & -62.21 (High) \\
\hline Delay (ms) & 100 (Low) \\
\hline Jitter (ms) & 10 (Low) \\
\hline PLR (loss per $10^{6}$ bytes) & 3 (Low) \\
\hline Throughput (Mlbps) & 130 (High) \\
\hline
\end{tabular}

Scenario 1: For the VHITS handoff necessity estimation, we assume that the enduser is currently watching a recorded webcast (streaming) using his/her own WLAN. Different parameters for this scenario are shown in Table 4.1.

A comparison of weights, calculated based on the different preference weighting mechanisms, is shown in Figure 4.1. The first-level criteria weights are assigned based on the user and/or operator preferences and are purely subjective in nature. To guarantee the continuity and the quality of the on-going session, higher weight assignments can be observed for RSS and QoS. A detailed description for the weight calculation process can be found in Chapter 3.

An observation that can be made from Figure 4.1 is that AHP tends to assign higher weights for RSS and QoS as compared with FAHP and TFN based weighting schemes. This is due to the fact that the pairwise comparisons between the attributes in 
AHP are performed using crisp values as compared with FAHP and TFN that utilize fuzzy numbers. A better distribution of weights among all parameters can be observed in TFN based scheme that relies on the usage of Linguistic Variables.

Figure 4.2 shows AHP-based weights assignments for different QoS parameters based on the characteristics of different traffic classes followed by Figures 4.3-4.4 that show the calculated weights for QoS parameters using FAHP and TFN-based weighting schemes, respectively. It can be seen that all three weighting schemes assign higher weight to throughput with respect to the Streaming class and higher weights to delay, and jitter for traffic based on Conversational class.

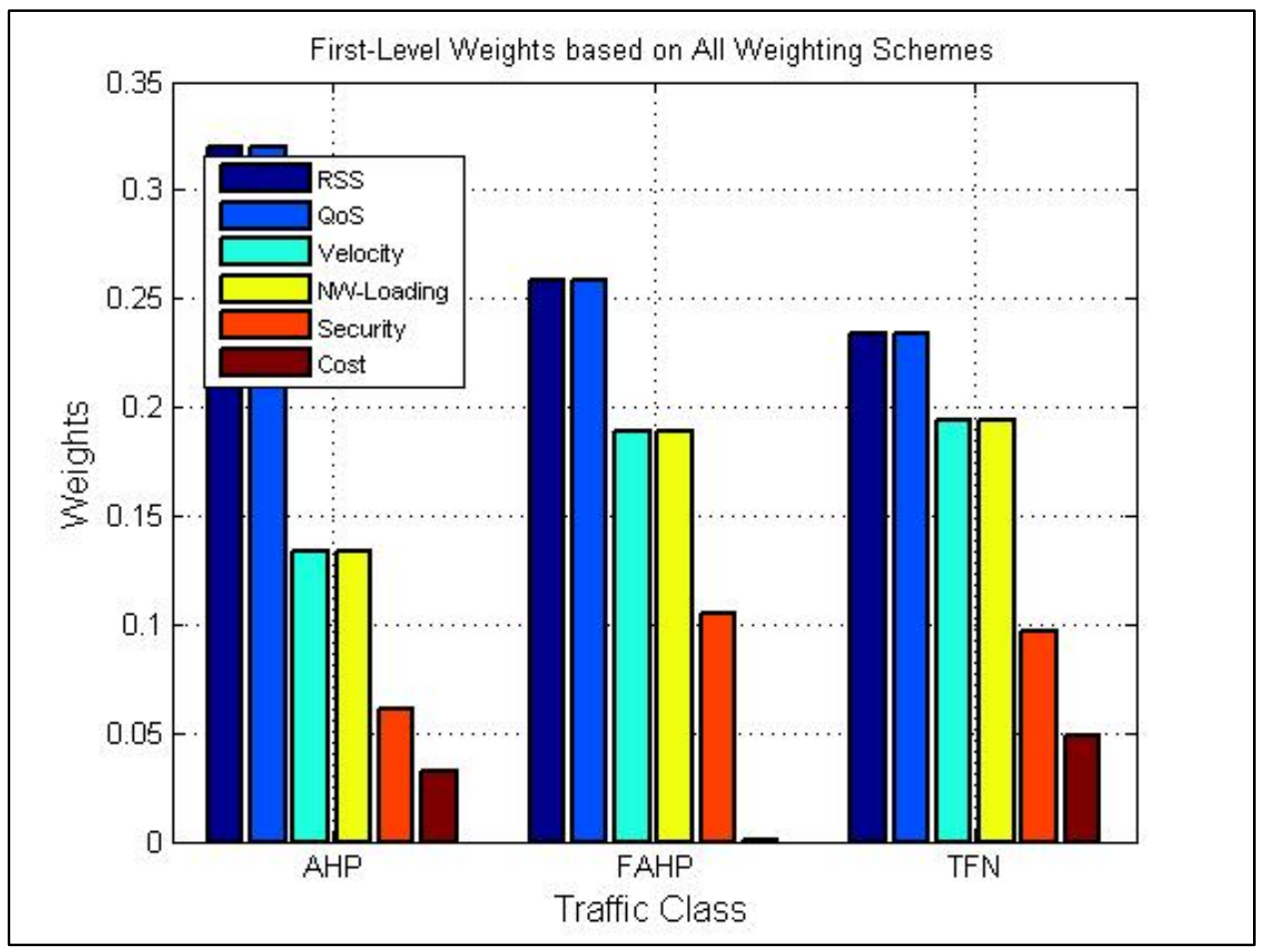

Figure 4.1: First-Level Weights based on All Weighting Schemes 


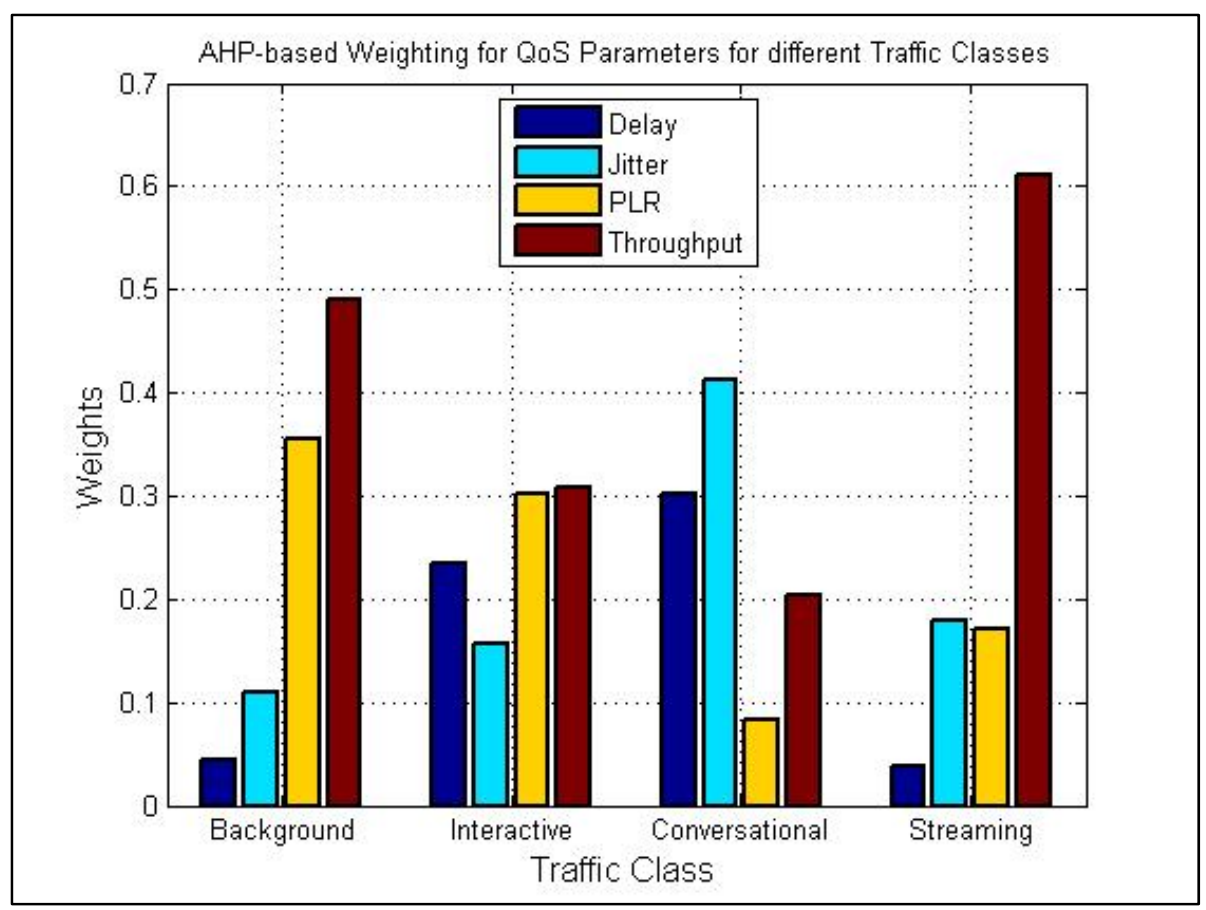

Figure 4.2: AHP-based Weights for QoS Parameters

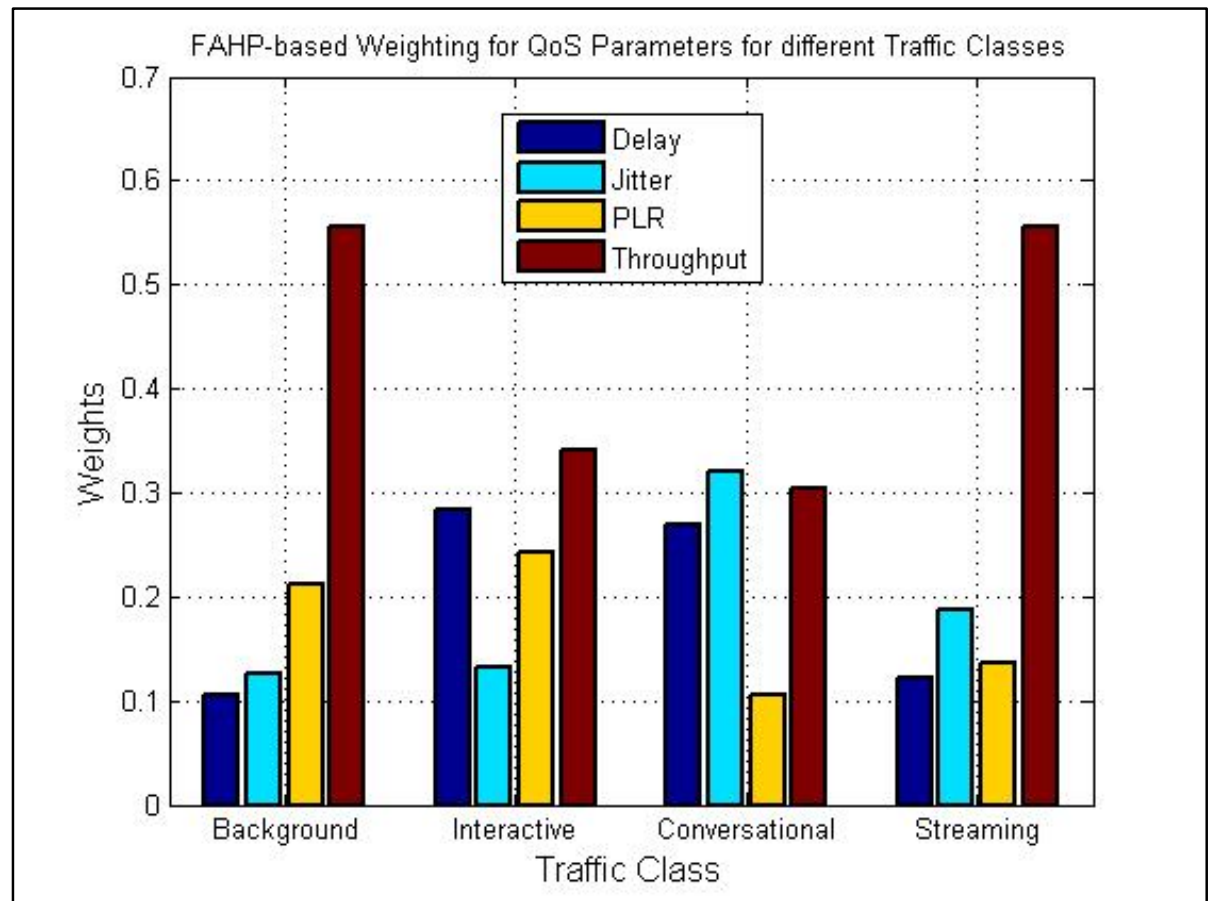

Figure 4.3: FAHP-based Weights for QoS Parameters 


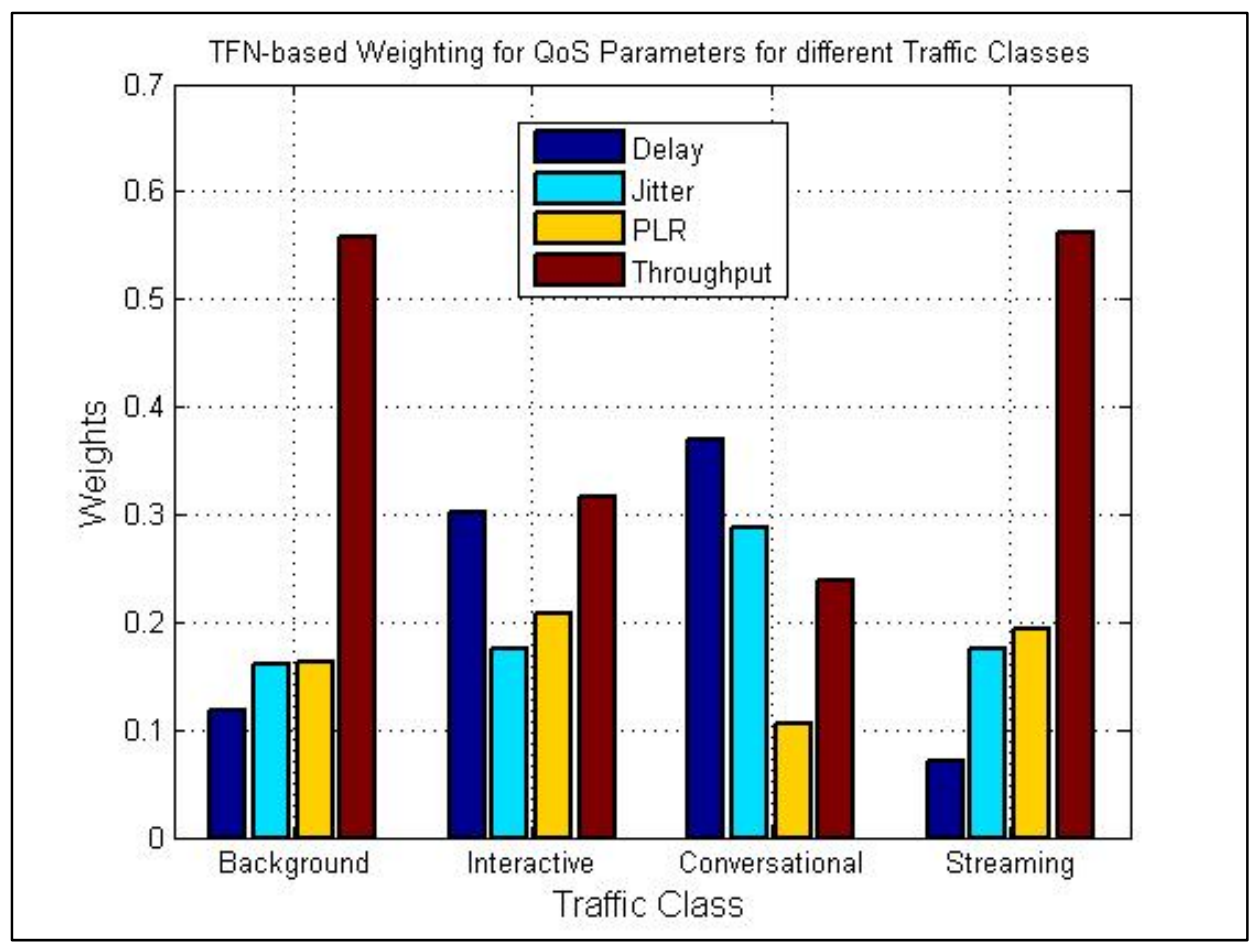

Figure 4.4: Linguistic-Variables based Weights for QoS Parameters

Based on the parameter values presented in Table 4.1, the overall handoff factor that the VHITS handoff necessity estimation module calculates comes out to be a low value of 0.25 . Since this value is less than the handoff threshold $(0.75)$, set for WLAN, the MS will not perform any handoff and will remain connected to its current PoA.

Scenario 2: Assuming that the end-user leaves home for work and starts walking toward the nearest bus stand while watching the same webcast, the distance between the WLAN-AP and MS increases and the RSS becomes weaker the further the user walks away from his/her home. The new parameter set is presented in Table 4.2. Note that based on the RSS samples, the GPT predicted an RSS value that cannot be sensed by the MS. In this scenario, the VHITS handoff necessity estimation calculates a handoff factor of 0.85 that is higher than the set handoff-threshold. Hence, the module will trigger the handoff and execute the VHITS target network selection module to find out the best 
available network that can support the continuity and the quality of the currently utilized service.

Table 4.2: New Parameter Set When User Walks Away from Current PoA

\begin{tabular}{|l|c|}
\multicolumn{1}{|c}{ Parameters } & Values \\
\hline Current PoA & WLAN \\
\hline Current Traffic Class & Streaming \\
\hline Metric Weight Scheme & AHP \\
\hline MS-Velocity (m/s) & 1 (Low) \\
\hline MS-PoA Distance (m) & 85 (Far) \\
\hline RSS Samples (dbm) & $-90.5,-92.7,-97.3,-98.9$ \\
\hline Predicted RSS using GPT (dbm) & -102.63 (Undetectable) \\
\hline Delay (ms) & 120 (High) \\
\hline Jitter (ms) & 20 (High) \\
\hline PLR (loss per 10 & bytes) \\
\hline Throughput (Mlbps) & 4 (Medium) \\
\hline
\end{tabular}

As the user is walking towards the bus stand, the VHITS handoff target network selection scheme senses the availability of three different networks. The parameter values for these three networks are presented in Table 4.3. These values are fed into the parallel FLCs of the VHITS handoff target network selection scheme. The FLCs normalize these parameters and produce their corresponding membership values based on whether the parameters are benefit or cost type. These normalized values are presented in Table 4.4. Note that the MS has different membership values for MS-Velocity corresponding to each available network. This is because the fuzzy logic rules are designed to assign higher speed MSs to WWAN while keeping the slower MSs to WLAN. Since the current walking velocity is $2 \mathrm{~m} / \mathrm{s}$, the FLCs estimate a lesser probability of rejection for an MS to 
be associated with the WLAN. A graphical representation of the normalized parameter values is shown in Figure 4.5.

Table 4.3: Parameter Set for Available Networks in-Range of Walking User

\begin{tabular}{|l|c|c|c|}
\hline \multicolumn{1}{|c|}{ Parameters } & WLAN & WMAN & WWAN \\
\hline PRSS (dbm) & -114.05 & -137.40 & -116.10 \\
\hline Delay (ms) & 130 & 20 & 10 \\
\hline Jitter (ms) & 27 & 5 & 4 \\
\hline PLR (loss per 10 bytes) $^{\text {(m) }}$ & 3 & 4 & 3 \\
\hline Throughput (Mbps) & 70 & 60 & 1.5 \\
\hline NW-Load (\%) & 20 & 30 & 40 \\
\hline Security (1-10) & 1 & 5 & 7 \\
\hline Cost (1-10) & 3 & 4 & 7 \\
\hline MS-Velocity (m/s) & & 2 & \\
\hline
\end{tabular}

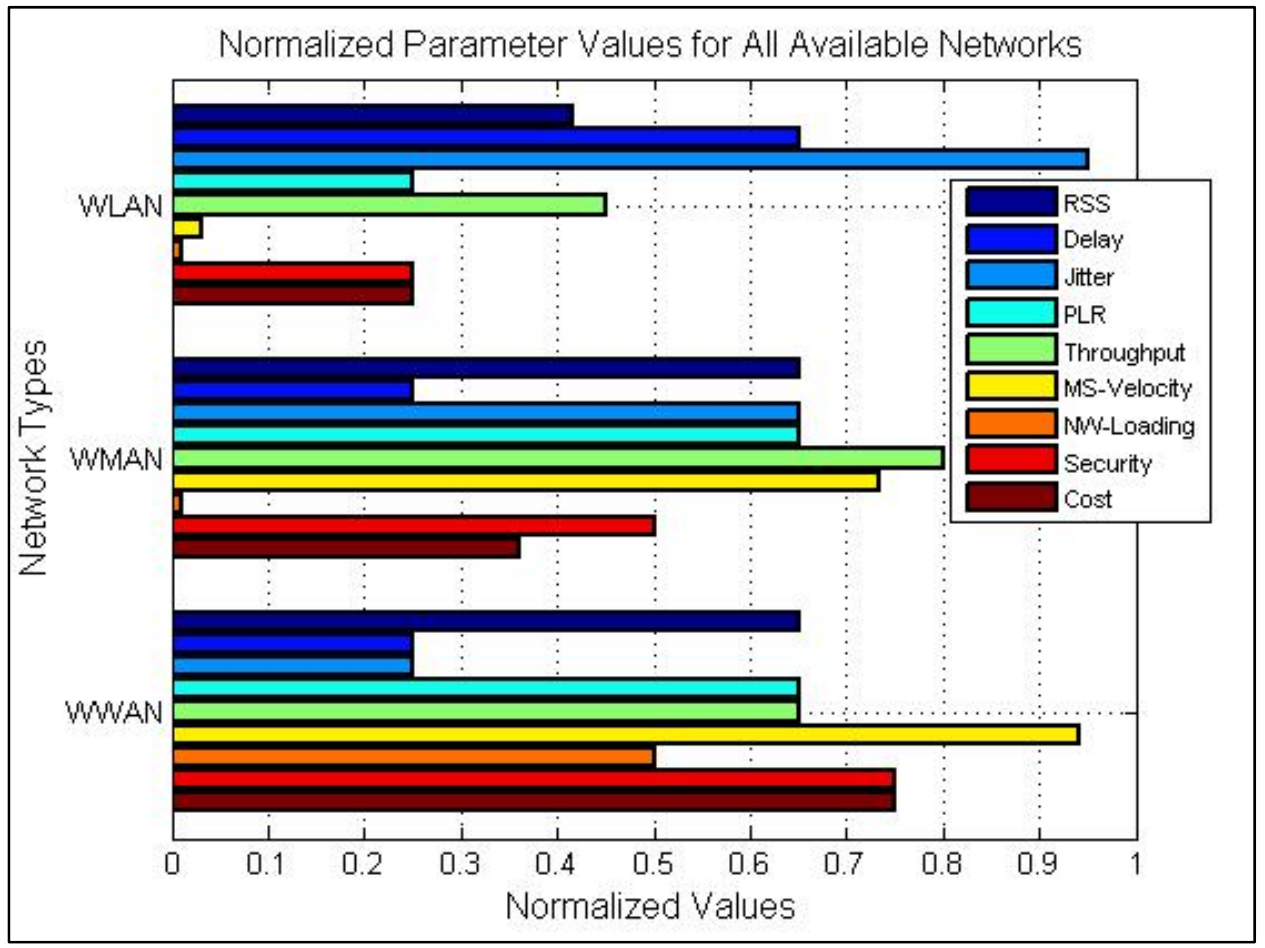

Figure 4.5: Normalized Networks Parameters (Velocity $=2 \mathrm{~m} / \mathrm{s}$ ) 
Table 4.4: Normalized Parameter Set and AHP based Weights for All Networks

\begin{tabular}{|l|c|c|c|c|}
\hline \multicolumn{1}{|c|}{ Parameters } & WLAN & WMAN & WWAN & (AHP) \\
\hline PRSS (dbm) & 0.42 & 0.65 & 0.65 & 0.3199 \\
\hline Delay (ms) & 0.65 & 0.25 & 0.25 & 0.0120 \\
\hline Jitter (ms) & 0.95 & 0.65 & 0.25 & 0.0574 \\
\hline PLR (per 10 bytes) & 0.25 & 0.65 & 0.65 & 0.0548 \\
\hline Throughput (Mbps) & 0.45 & 0.80 & 0.65 & 0.1957 \\
\hline MS-Velocity (m/s) & 0.03 & 0.73 & 0.94 & 0.1337 \\
\hline NW-Load (\%) & 0.01 & 0.01 & 0.50 & 0.1337 \\
\hline Security (1-10) & 0.25 & 0.50 & 0.75 & 0.0607 \\
\hline Cost (1-10) & 0.25 & 0.36 & 0.75 & 0.0320 \\
\hline
\end{tabular}

In order to select the best target network which maximizes the end-user satisfaction in terms of service continuity and QoS, the decision matrix, comprising of normalized values from Table 4.4 along with their associated weights, are fed into the TOPSIS ranking algorithm. Figure 4.6 shows the ranking values for all available networks produced by TOPSIS for all four traffic classes. It can be observed that based on the selected values of network parameters and their corresponding weights, an MS moving with a velocity of $2 \mathrm{~m} / \mathrm{s}$ prefers WMAN for Streaming and Conversational traffic classes. On the other hand, for Interactive and Background traffic classes, connecting to WLAN would fulfill end-user's requirements; a higher throughput is required for the Streaming traffic class and Conversational traffic class requires smaller values of end-to- 
end delay and jitter. A strong competition between WLAN and WMAN, for Conversational and Streaming traffic classes, can also be observed. As can be seen from Figure 4.5 and Table 4.4, WMAN fulfills these requirements for both classes.

Although a weaker RSS can be seen for WLAN, other factors like velocity, network-loading and network-cost, influence TOPSIS to select WLAN as the preferred network for Interactive and Background traffic classes.

Scenario 3: Assuming that the same user, who is watching the webcast, steps in a bus that starts to move with a relatively higher velocity than that of walking user. We will provide numerical examples for two different velocities. Although, RSS and some other parameters do not remain constant and changes rapidly due to the dynamic nature of wireless networks, we will keep these values constant just to focus on the effects of velocity on the network selection process. Note that in Sections 4.3-4.5 we will provide results, taking into consideration the dynamic behavior of wireless networks.

Figure 4.7 shows the membership values for all the network parameters when the bus is moving at a velocity of $5 \mathrm{~m} / \mathrm{s}$. It can be seen that the probability of rejection for an MS to be associated with WLAN has now increased, whereas for WMAN and WWAN it is relatively lower. This is because the inference rules for FLC-1 are designed to select WMAN or WWAN for higher speed MSs. Consequently, TOPSIS prefers WMAN and WWAN for all traffic classes as can be observed from Figure 4.8. For Streaming, Background, and Interactive traffic classes, TOPSIS prefers WMAN over WWAN. A close competition can be observed between both networks for Interactive class. As the Conversational traffic class does not tolerate higher values of delay and jitter, TOPSIS 
prefers to choose WWAN over WMAN; a relatively lower value of delay and jitter are provided by WWAN as can be seen from Figure 4.7.

A higher velocity MS moving through a network with a smaller coverage area performs frequent handoffs. Since WWAN provides a larger coverage area than WLAN and WMAN, the FLC-1 is designed to associate higher speed MSs to WWAN to avoid frequent handoffs and to preserve the continuity of the current session. The rankings of preferred networks for different traffic classes, when the bus moves at a velocity of 10 $\mathrm{m} / \mathrm{s}$, are presented in Figure 4.9. Based on the above explanation, TOPSIS prefers WWAN over WMAN, and WLAN.

Figures 4.10-4.12 show TOPSIS rankings for the same scenarios discussed above, but utilizing the FAHP weighting scheme. By utilizing FAHP weighting method, uncertainty and vagueness from subjective perceptions of user's preferences can be effectively represented. For the MS with a velocity of $2 \mathrm{~m} / \mathrm{s}$, TOPSIS with FAHP prefers WLAN for all types of traffic classes. When the MS is moving at $5 \mathrm{~m} / \mathrm{s}$, the preference is WMAN. The WWAN is preferred by TOPSIS at much higher velocities.

The rankings for all networks utilizing TOPSIS with the TFN weighting scheme for MSs moving with different speeds are presented in Figures 4.13-4.15. A similar trend, like FAHP weighting scheme, can be observed where the preferred network is WLAN for slower moving MSs and WWAN for MSs moving with higher speeds. However, Figure 4.13 shows a close competition between WLAN and WMAN for MSs moving with a velocity of $2 \mathrm{~m} / \mathrm{s}$. A similar competition can be observed between WMAN and WWAN for MSs moving with $5 \mathrm{~m} / \mathrm{s}$. This can be observed from Figure 4.14. 


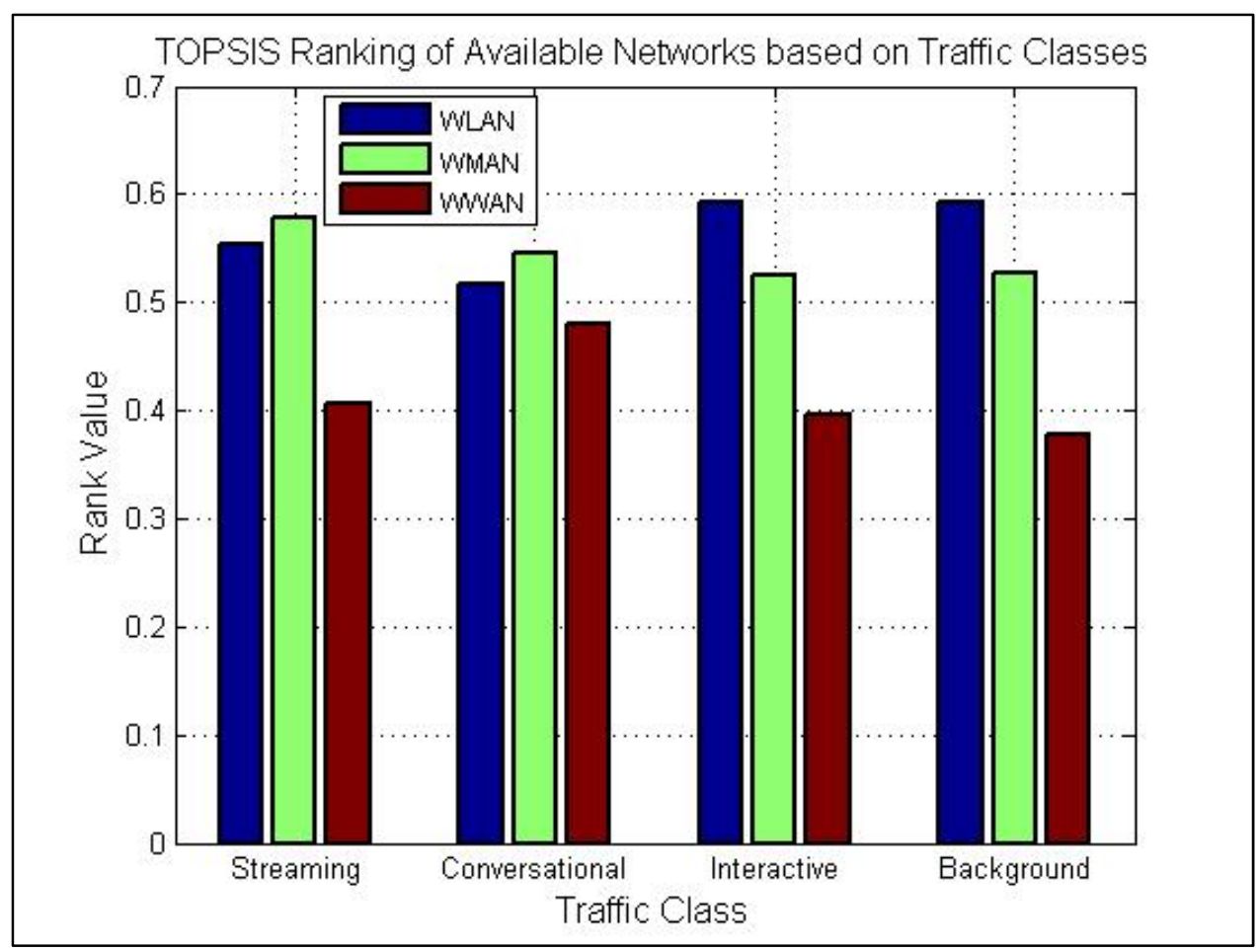

Figure 4.6: TOPSIS Ranking of Networks based on Traffic Classes and AHP weighting (Velocity $=2 \mathrm{~m} / \mathrm{s}$ )

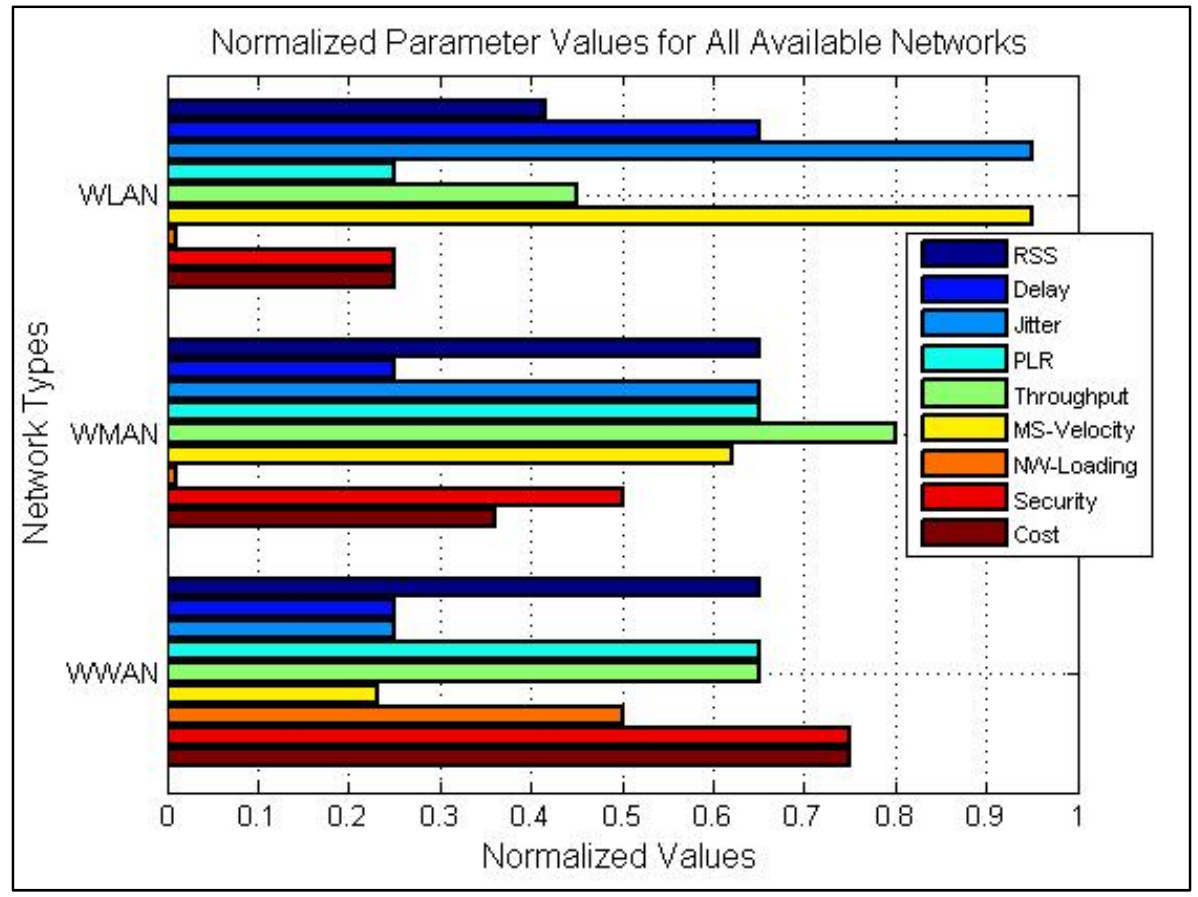

Figure 4.7: Normalized Networks Parameters (Velocity $=5 \mathrm{~m} / \mathrm{s}$ ) 


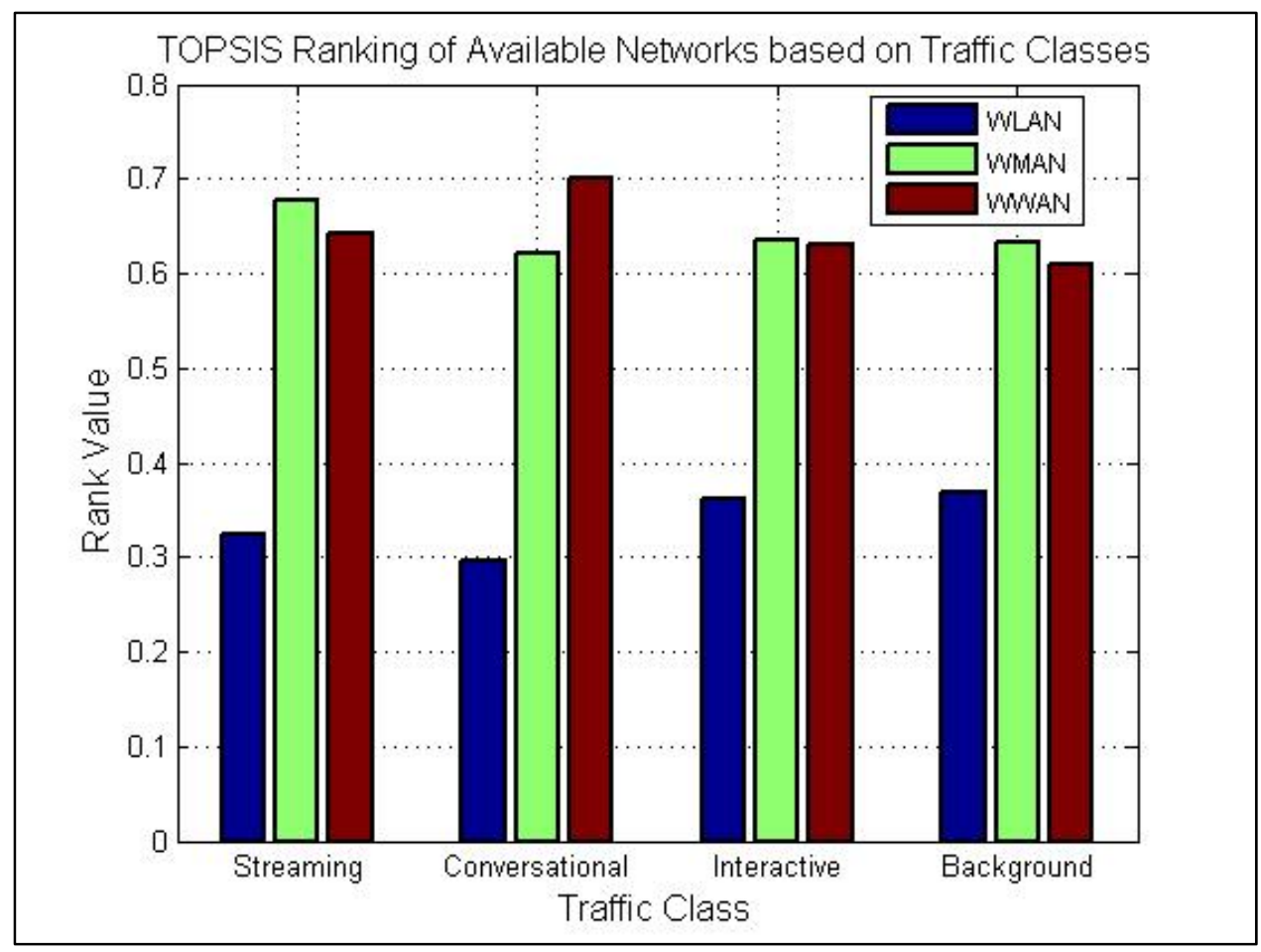

Figure 4.8: TOPSIS Ranking of Networks based on Traffic Classes and AHP weighting $($ Velocity $=5 \mathrm{~m} / \mathrm{s}$ )

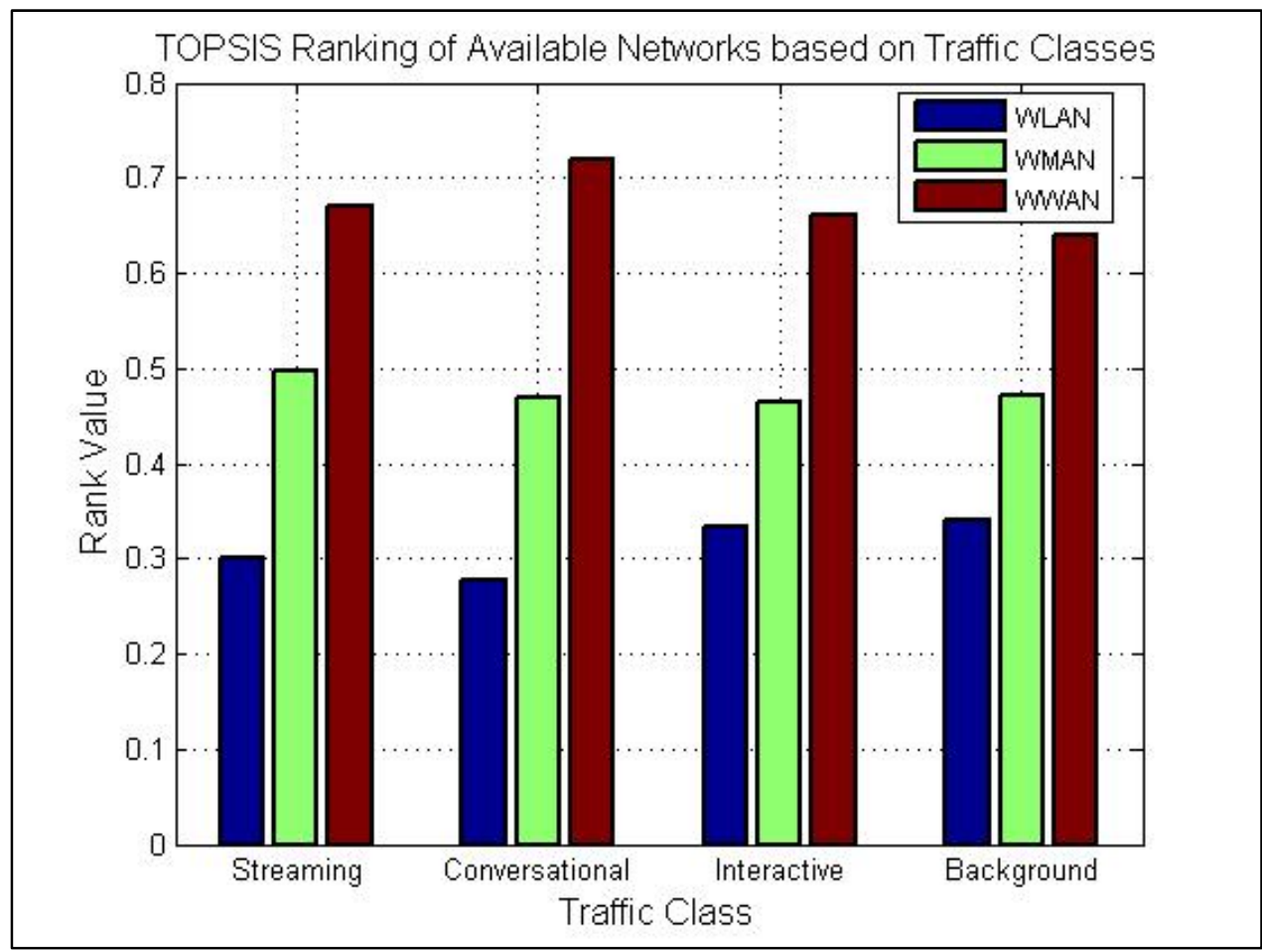

Figure 4.9: TOPSIS Ranking of Networks based on Traffic Classes and AHP weighting $($ Velocity $=10 \mathrm{~m} / \mathrm{s}$ ) 


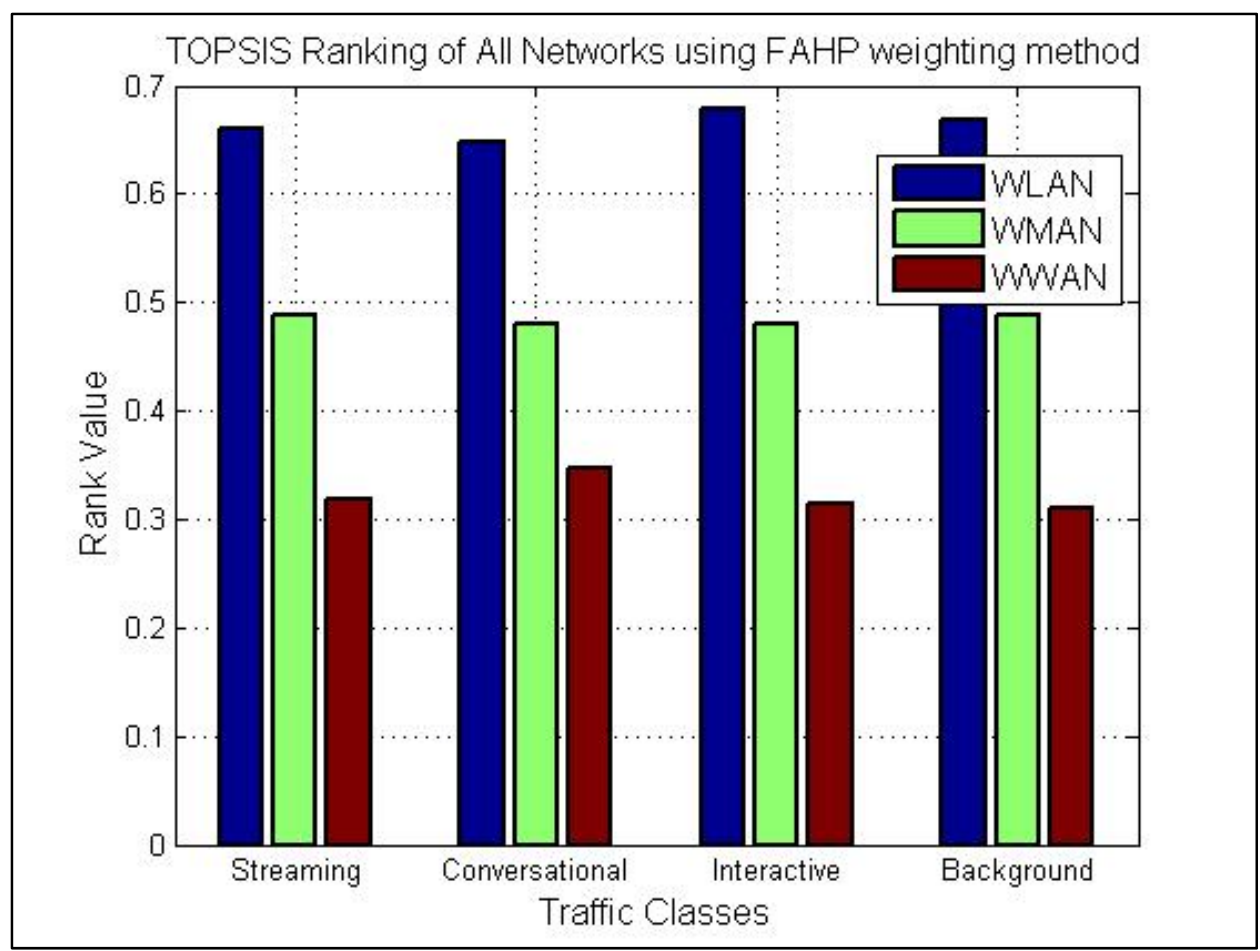

Figure 4.10: TOPSIS Ranking of Networks based on Traffic Classes and FAHP weighting (Velocity $=2 \mathrm{~m} / \mathrm{s}$ )

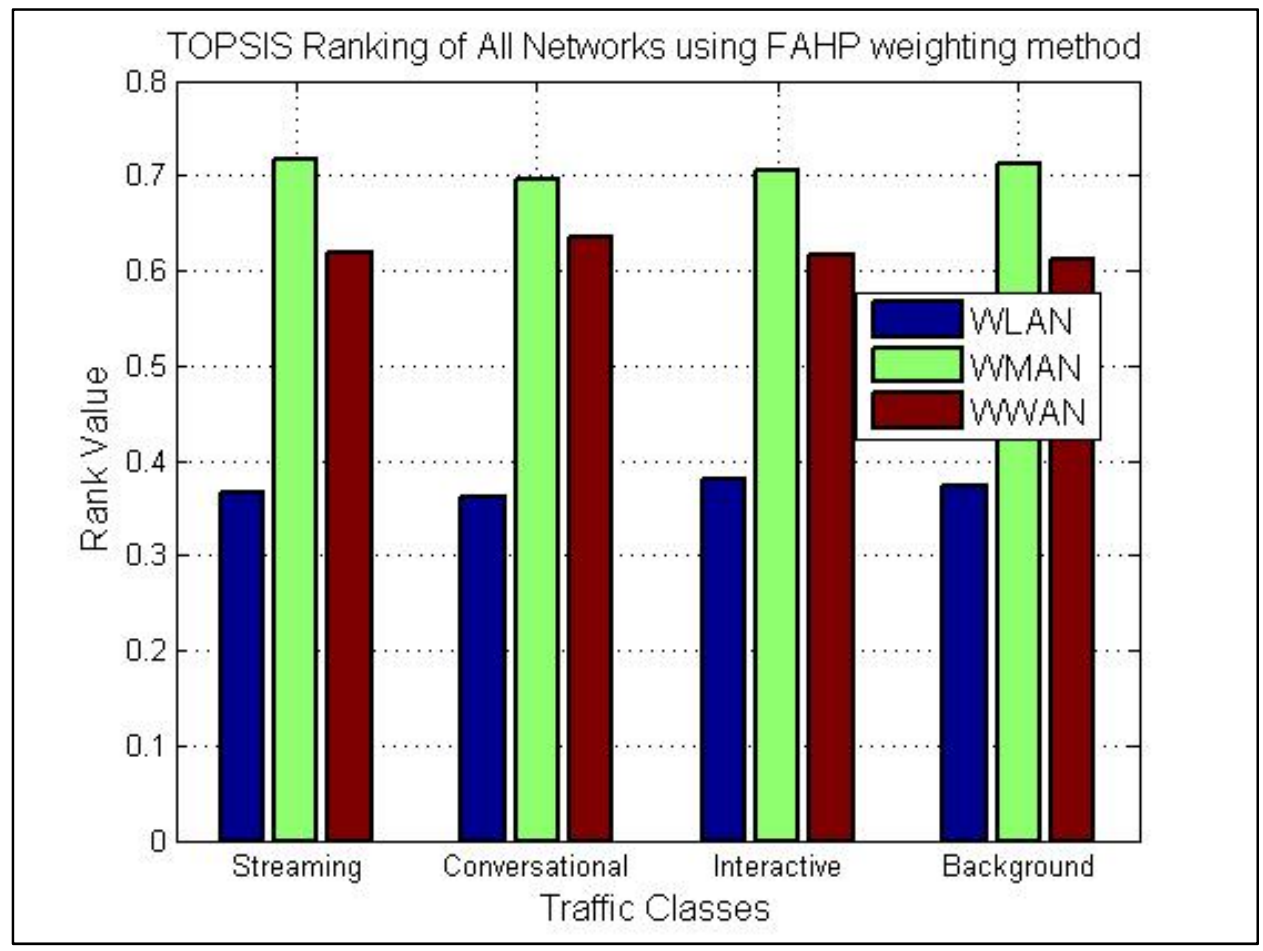

Figure 4.11: TOPSIS Ranking of Networks based on Traffic Classes and FAHP weighting (Velocity $=5 \mathrm{~m} / \mathrm{s}$ ) 


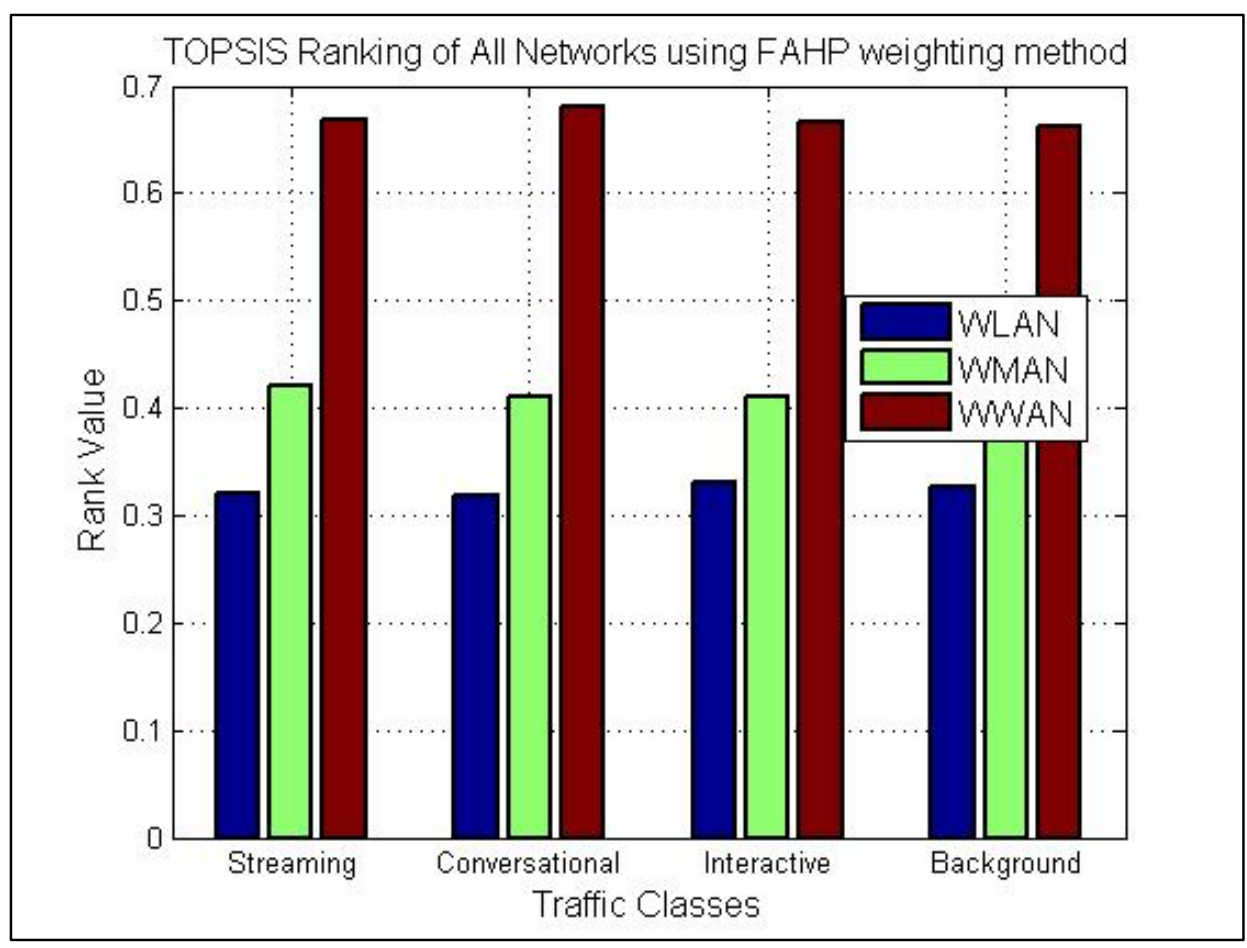

Figure 4.12: TOPSIS Ranking of Networks based on Traffic Classes and FAHP weighting (Velocity $=10 \mathrm{~m} / \mathrm{s}$ )

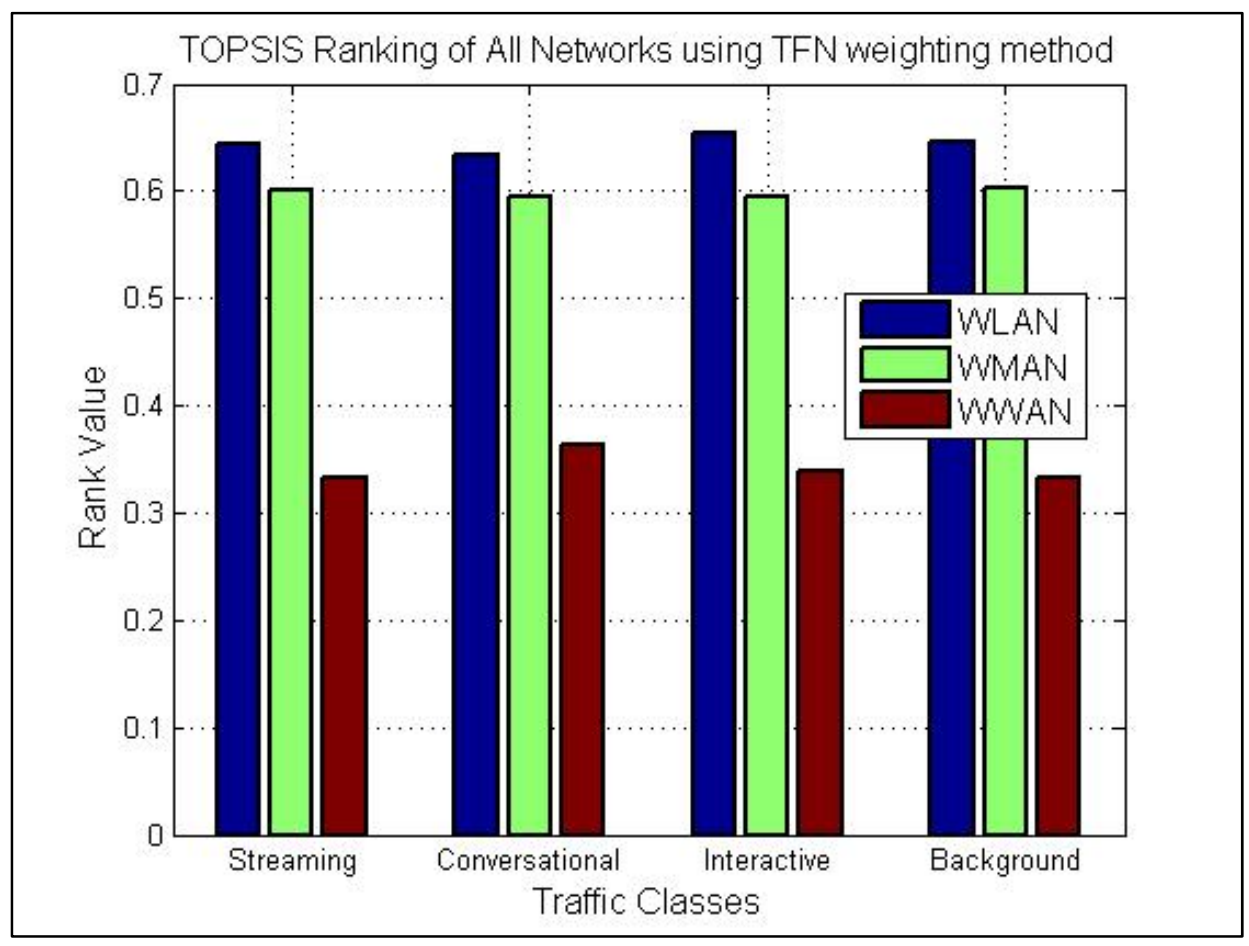

Figure 4.13: TOPSIS Ranking of Networks based on Traffic Classes and TFN weighting (Velocity $=2 \mathrm{~m} / \mathrm{s}$ ) 


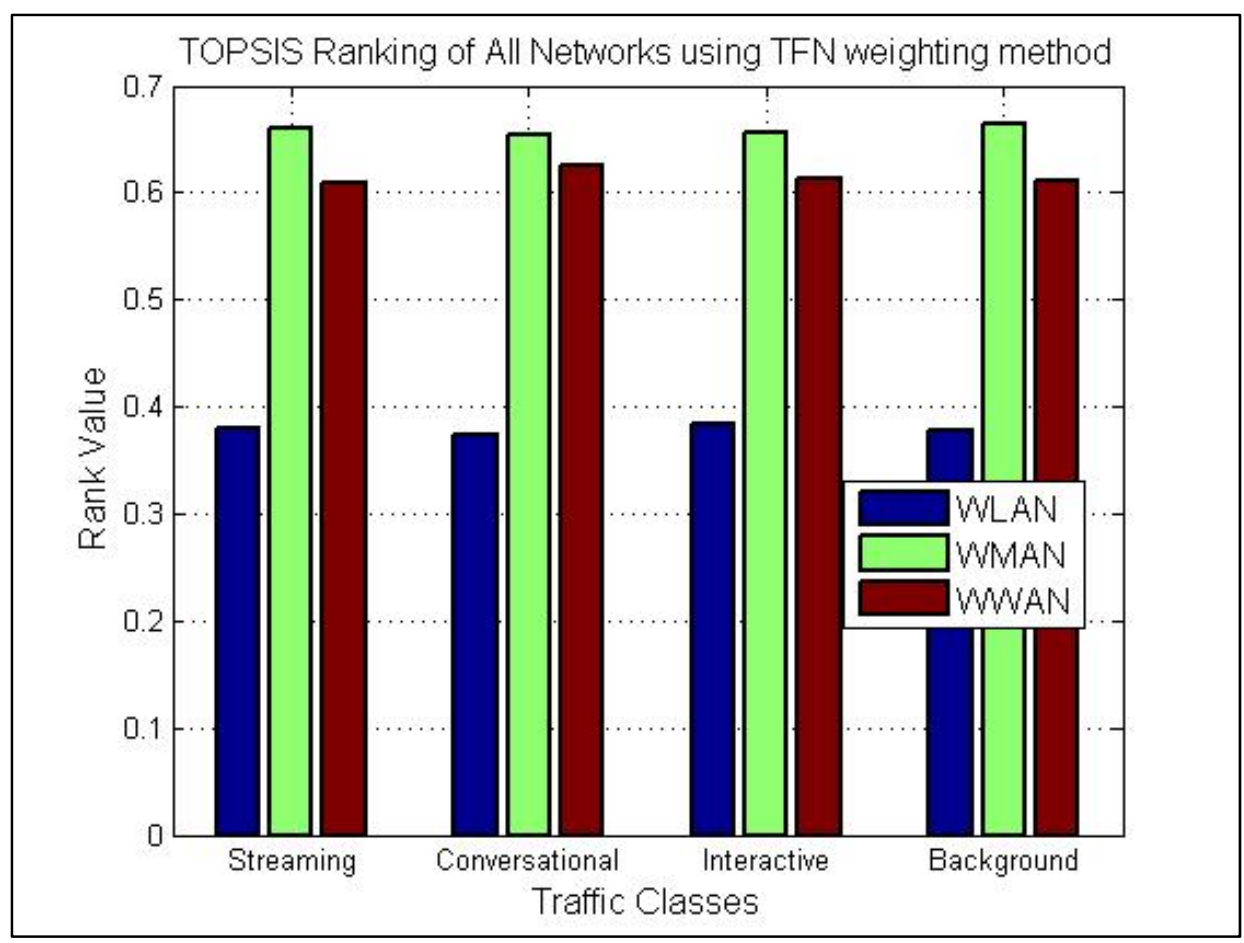

Figure 4.14: TOPSIS Ranking of Networks based on Traffic Classes and TFN weighting (Velocity $=5 \mathrm{~m} / \mathrm{s}$ )

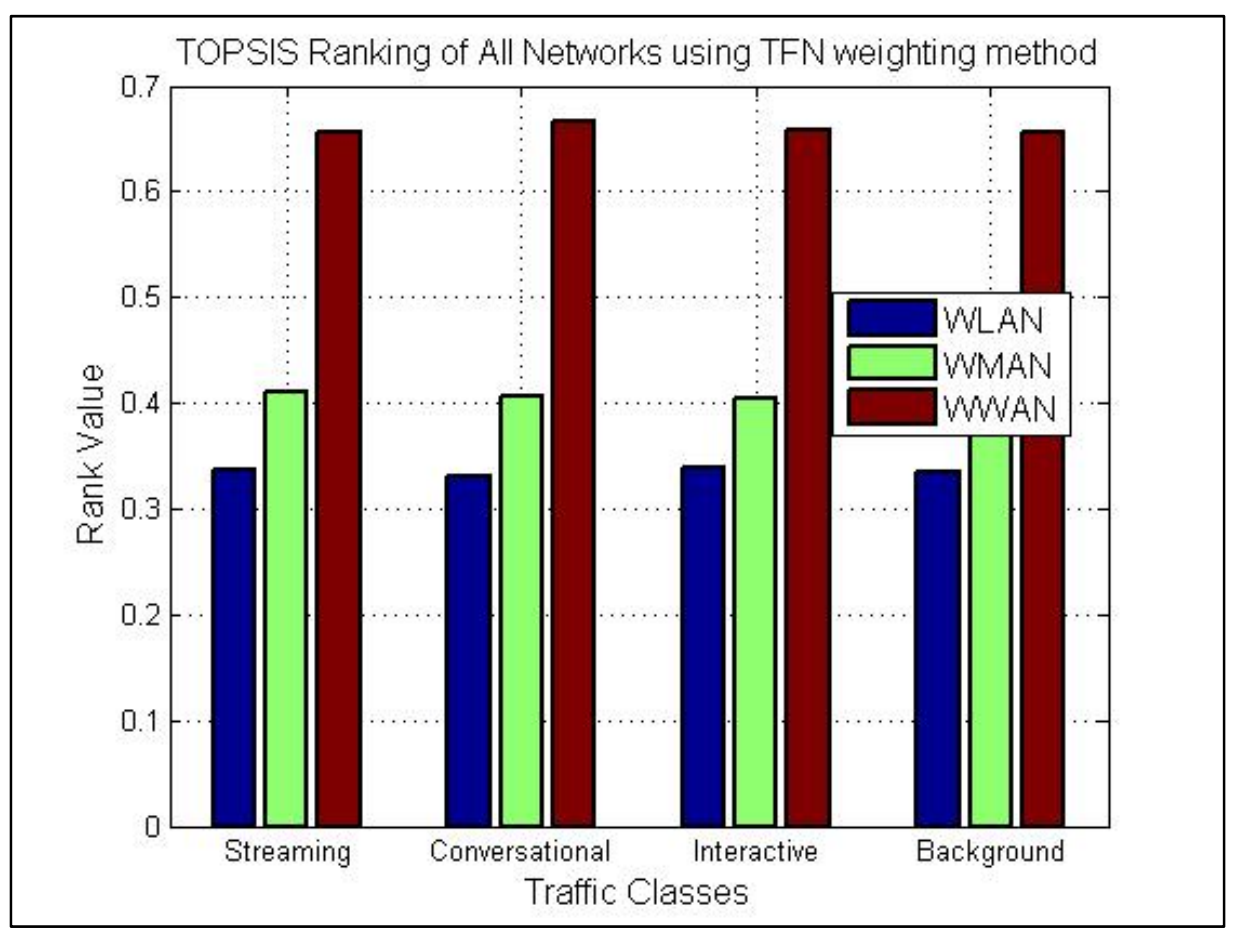

Figure 4.15: TOPSIS Ranking of Networks based on Traffic Classes and TFN weighting (Velocity $=10 \mathrm{~m} / \mathrm{s}$ ) 
In a possible situation where a preferred network fails to provide an available channel to MS, the VHITS handoff target network selection scheme is intelligent enough to select the second or third preferred network as the target of handoff. The parameter values used to generate rankings in Figure 4.16 are exactly the same as used in Figure 4.8 , with the exception of Network-Loading for WWAN network. Since there are no available channels on WWAN network, the VHITS target selection scheme selects WMAN as the preferred network; WMAN is ranked second in Figure 4.8. In case of both WWAN and WMAN not being available, as shown in Figure 4.17, WLAN is the target network.

We will complete this section by providing numerical examples, results, and brief discussion for two network selection schemes, namely FTOPSIS and FVIKOR, where both parameter values measured from networks and their associated weights are treated as fuzzy values. Similar values of parameters as depicted in Table 4.3 are used to generate results. The only exception is MS-speed that is set at $5 \mathrm{~m} / \mathrm{s}$.

Tables 4.5-4.6 show the decision matrix containing network attributes and their associated weights, respectively. The network attributes are represented using TFNs, and Linguistic Variables are used to represent their weights. The decision and weight matrices are then used as inputs to the FTOPSIS scheme. The normalized weighted decision matrix for the Streaming traffic class is shown in Table 4.7. This table also contains the FPIS $\left(A^{+}\right)$and FNIS $\left(A^{-}\right)$for the Streaming traffic class. Similar tables can be constructed for the other three traffic classes as well. Note, the FPIS $\left(A^{+}\right)$and FNIS $\left(A^{-}\right)$ values can also be set manually as $(1,1,1)$ for benefit and $(0,0,0)$ for cost criterion, respectively. 


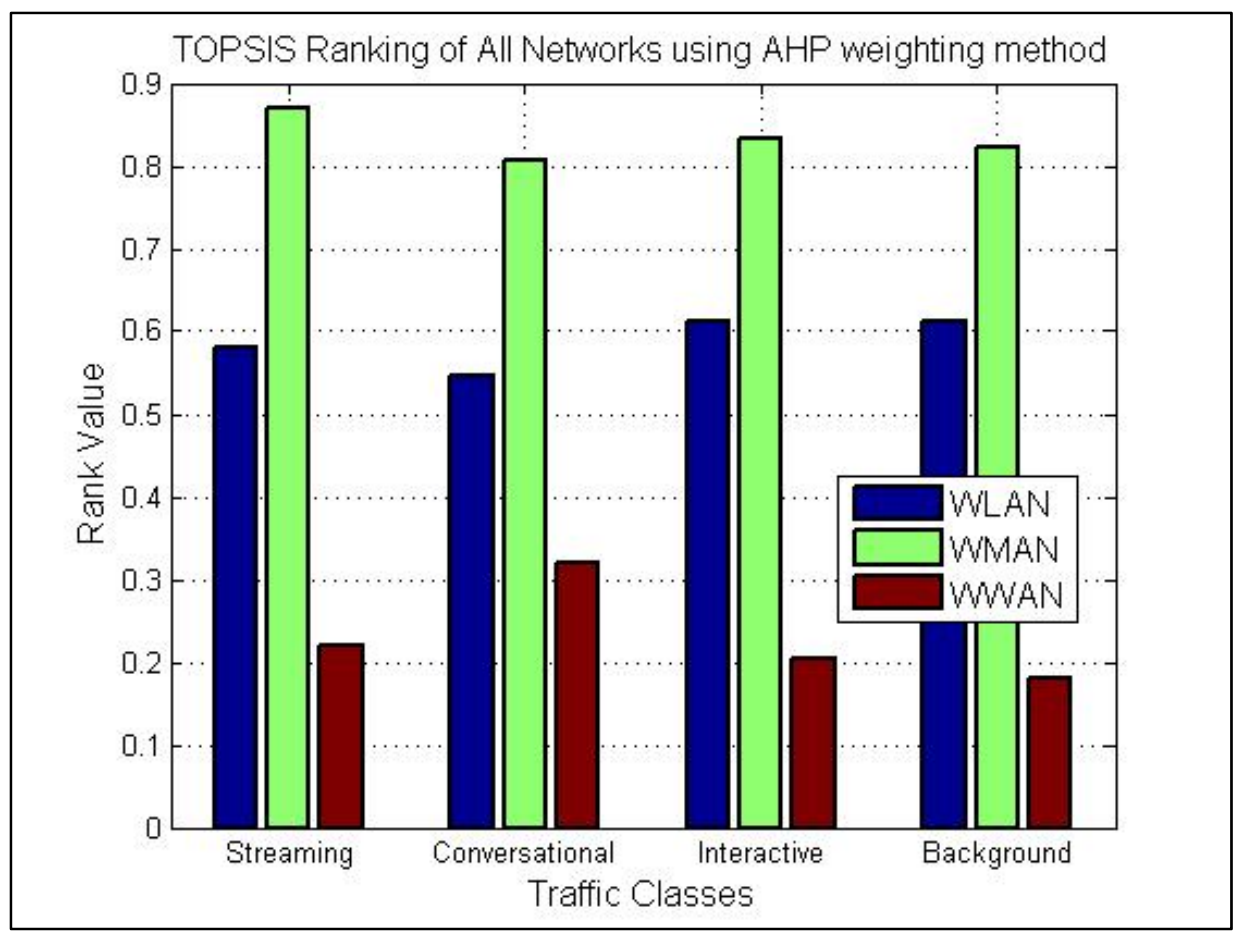

Figure 4.16: TOPSIS Ranking of Networks with AHP weighting $($ Velocity $=5 \mathrm{~m} / \mathrm{s}$, WWAN-Loading $=100 \%)$

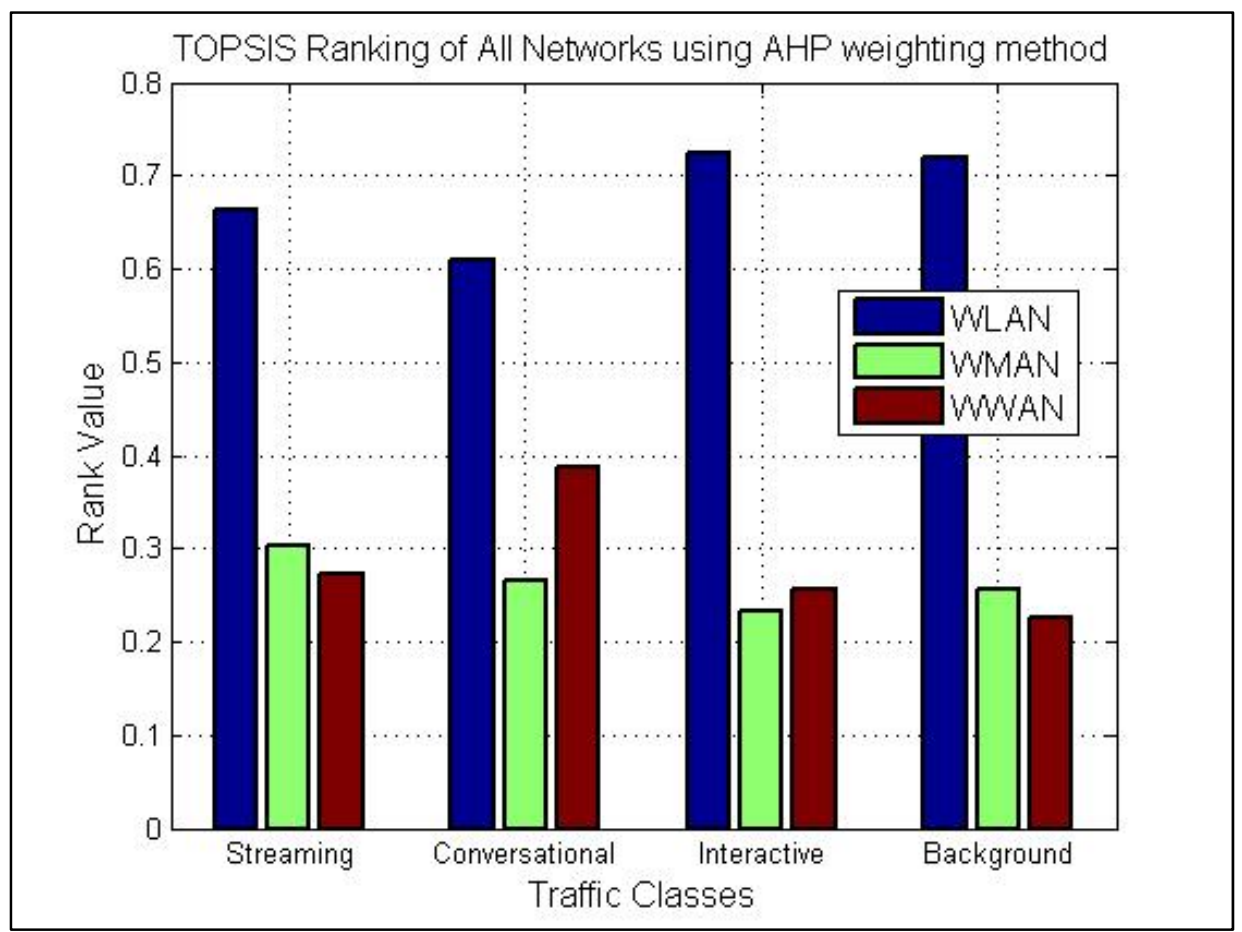

Figure 4.17: TOPSIS Ranking of Networks with AHP weighting

$($ Velocity $=5 \mathrm{~m} / \mathrm{s}$, WWAN-Loading $=100 \%$, WMAN-Loading $=100 \%)$ 
Table 4.8 shows different calculations required for FTOPSIS for all traffic classes and available networks. These include the calculations of distances between each alternatives and FPIS \& FNIS, which are given by $d\left(A_{i}, A^{+}\right)$and $d\left(A_{i}, A^{-}\right)$, respectively. The table also shows, for each alternative, the Closeness Coefficients from the ideal solution, which determine the final rankings of the available networks. These rankings are depicted in Figure 4.18 where it can be seen that WMAN outperforms all other networks for all four traffic classes.

The calculations for FVIKOR ranking scheme is shown in Tables 4.9-4.10. Fuzzy Best Values (FBV) $\left(\tilde{f}_{j}^{+}\right)$and Fuzzy Worst Values (FWV) $\left(\tilde{f}_{j}^{-}\right)$for Streaming traffic class are calculated that are also provided in the same table. FBV and FWV for other traffic classes can be generated in a similar fashion. Table 4.10 shows all the numerical calculations required for FVIKOR ranking algorithm. The triangular fuzzy number $\tilde{Q}_{i}$ is then defuzzified into a crisp number. The Network with the smallest value of $Q_{i}$ is chosen as the target network; smaller value implies better performance of a candidate. These network rankings are presented in Figure 4.19 for all types of traffic classes. Note that only ranking number of the preferred network is displayed in this figure. Hence, the network preferred by FVIKOR for an MS moving with a velocity of $5 \mathrm{~m} / \mathrm{s}$, is WMAN with a ranking of 1. WWAN and WLAN are second and third choices.

\subsection{SIMULATION SETUP AND ENVIRONMENT}

The VHITS handoff necessity estimation and target selection schemes are implemented in MATLAB (R2011a). Fuzzy Logic toolbox is used to implement the different FLCs that are used in this research work. The developed scheme is evaluated 
using a test-bed created based on the concept of RUNE [134]. RUNE, originated as a research project at Ericsson, is a special purpose simulator to simulate heterogeneous wireless networks. It is well verified and extensively used in the field of wireless communications [29, 91, 135-137]. Several models, including system, mobility, propagation, and traffic, are created using RUNE. In the following, a brief explanation regarding the implementation of these models is provided.

System Model: The system model defines the details of different types of networks. These details include, but are not limited to, the number and characteristics of the cells that constitutes a specific type of network. To simulate the proposed scheme, a system model is created with several co-existing WLANs, WMANs, and WWANs.

The WLAN is defined with 27 cells, each with a radius of 100 meters. The WMAN and WWAN are defined with 12 cells, each with a radius of 375 and 750 meters, respectively. The standard hexagonal shape with omni-directional antennas is considered for each cell for all three network types. A cluster of 3 cells is formed and the total frequency range for each network is divided among these 3 cells. These divided frequencies are repeated at each cluster. This arrangement is kept the same for all three network types. The total number of available channels per cell is kept as 8,12 , and 16 , for WLAN, WMAN, and WWAN, respectively. All channels are assumed to be orthogonal. The "wrap-around" function of RUNE is utilized to avoid cell boundaries for different coverage areas. This is necessary to make sure that the MS cannot move over the edge outside the coverage area and if they move, the adjacent service area automatically covers them. 


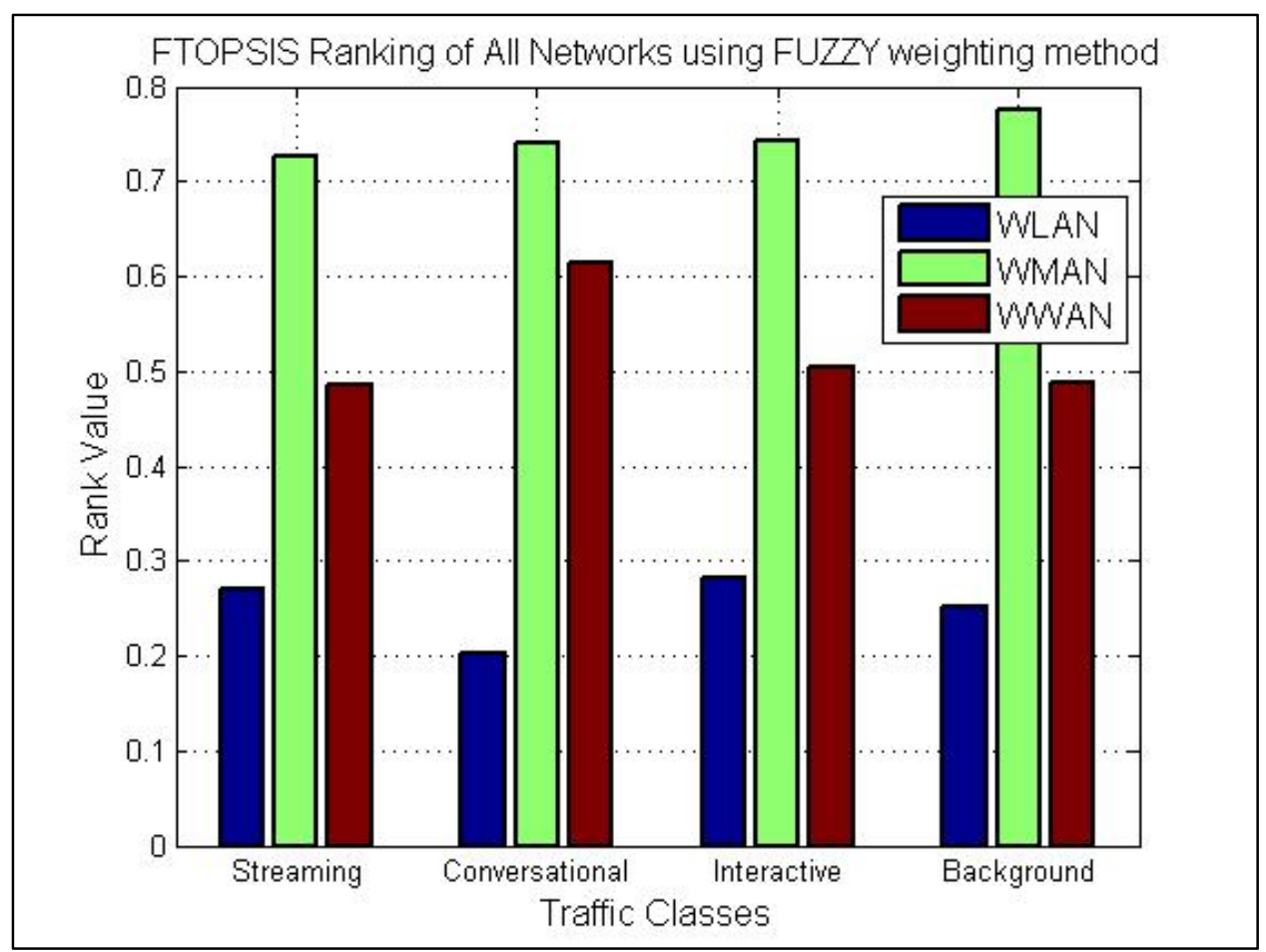

Figure 4.18: FTOPSIS Ranking for all traffic types and Available Networks $($ Velocity $=5 \mathrm{~m} / \mathrm{s})$

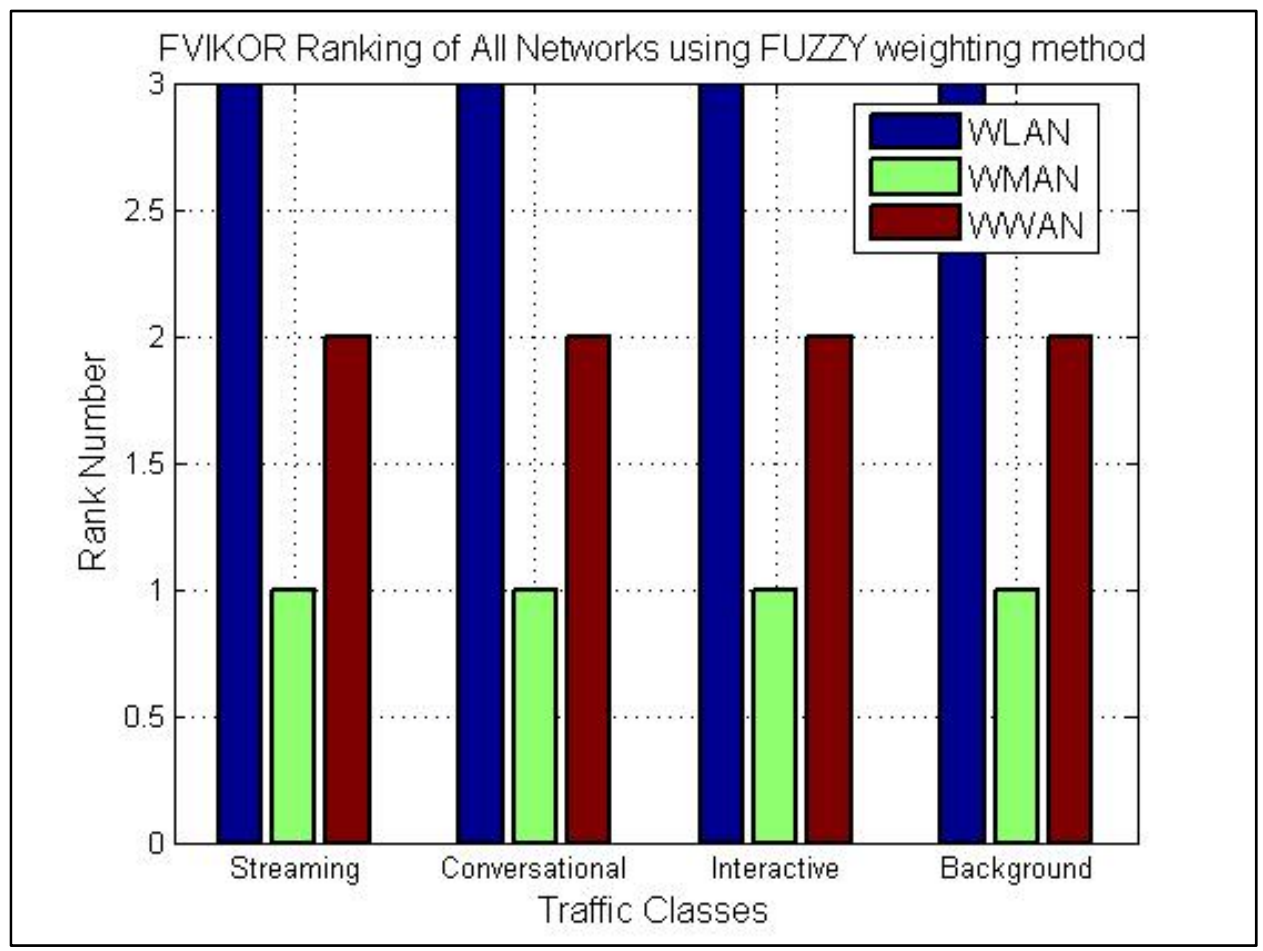

Figure 4.19: FVIKOR Network Rankings for all Traffic classes (Velocity $=5 \mathrm{~m} / \mathrm{s}$ ) 
Table 4.5: Fuzzy Decision Matrix for FTOPSIS

\begin{tabular}{|c|c|c|c|c|c|c|c|c|c|}
\hline Networks & RSS & Delay & Jitter & PLR & T.put & Velocity & Loading & Security & Cost \\
\hline WLAN & {$[0.2,0.4,0.6]$} & {$[0.4,0.6,0.8]$} & {$[0.8,1.0,1.0]$} & {$[0.0,0.2,0.4]$} & {$[0.2,0.4,0.6]$} & {$[0.8,1.0,1.0]$} & {$[0.0,0.2,0.4]$} & {$[0.0,0.2,0.4]$} & {$[0.0,0.2,0.4]$} \\
\hline WMAN & {$[0.4,0.6,0.8]$} & {$[0.0,0.2,0.4]$} & {$[0.4,0.6,0.8]$} & {$[0.4,0.6,0.8]$} & {$[0.6,0.8,1.0]$} & {$[0.0,0.2,0.4]$} & {$[0.0,0.2,0.4]$} & {$[0.2,0.4,0.6]$} & {$[0.2,0.4,0.6]$} \\
\hline WWAN & {$[0.4,0.6,0.8]$} & {$[0.0,0.2,0.4]$} & {$[0.0,0.2,0.4]$} & {$[0.4,0.6,0.8]$} & {$[0.4,0.6,0.8]$} & {$[0.4,0.6,0.8]$} & {$[0.2,0.4,0.6]$} & {$[0.6,0.8,1.0]$} & {$[0.6,0.8,1.0]$} \\
\hline
\end{tabular}

Table 4.6: Linguistic Weights used with FTOPSIS for Different Traffic Classes

\begin{tabular}{|c|c|c|c|c|c|c|c|c|c|}
\hline Parameter & RSS & Delay & Jitter & PLR & Throughput & Velocity & Loading & Security & Cost \\
\hline Streaming & E & L & M & VH & E & VH & H & M & L \\
\hline Conversational & E & E & VH & M & L & VH & H & M & L \\
\hline Interactive & E & VH & L & VH & M & VH & H & M & L \\
\hline Background & E & L & L & M & H & VH & H & M & L \\
\hline
\end{tabular}


Table 4.7: Normalized weighted Matrix, Streaming Class, showing FPIS and FNIS

\begin{tabular}{|c|c|c|c|c|c|c|c|c|c|}
\hline & RSS & Delay & Jitter & PLR & T.put & Velocity & Loading & Security & Cost \\
\hline WLAN & {$[0.16,0.4,0.6]$} & {$[0.0,0.12,0.32]$} & {$[0.16,0.4,0.6]$} & {$[0.0,0.16,0.4]$} & {$[0.16,0.4,0.6]$} & {$[0.48,0.8,1.0]$} & {$[0.0,0.0,0.16]$} & {$[0.0,0.08,0.24]$} & {$[0.0,0.04,0.16]$} \\
\hline WMAN & {$[0.32,0.6,0.8]$} & {$[0.0,0.04,0.16]$} & {$[0.08,0.24,0.48]$} & {$[0.24,0.48,0.8]$} & {$[0.48,0.8,1.0]$} & {$[0.0,0.0,0.2]$} & {$[0.0,0.0,0.16]$} & {$[0.04,0.16,0.36]$} & {$[0.0,0.08,0.24]$} \\
\hline WWAN & {$[0.32,0.6,0.8]$} & {$[0.0,0.04,0.16]$} & {$[0.0,0.08,0.24]$} & {$[0.24,0.48,0.8]$} & {$[0.32,0.6,0.8]$} & {$[0.24,0.48,0.8]$} & {$[0.08,0.24,0.48]$} & {$[0.12,0.32,0.6]$} & {$[0.0,0.16,0.4]$} \\
\hline FPIS $\left(A^{+}\right)$ & {$[0.32,0.6,0.8]$} & {$[0.0,0.04,0.16]$} & {$[0.0,0.08,0.24]$} & {$[0.0,0.16,0.4]$} & {$[0.48,0.8,1.0]$} & {$[0.0,0.0,0.2]$} & {$[0.0,0.0,0.16]$} & {$[0.12,0.32,0.6]$} & {$[0.0,0.04,0.16]$} \\
\hline FNIS $\left(A^{-}\right)$ & {$[0.16,0.4,0.6]$} & {$[0.0,0.12,0.32]$} & {$[0.16,0.4,0.6]$} & {$[0.24,0.48,0.8]$} & {$[0.16,0.4,0.6]$} & {$[0.48,0.8,1.0]$} & {$[0.08,0.24,0.48]$} & {$[0.0,0.08,0.24]$} & {$[0.0,0.16,0.4]$} \\
\hline
\end{tabular}

Table 4.8: FTOPSIS Calculations for All Traffic Classes

\begin{tabular}{|c|c|c|c|c|c|c|c|c|c|c|}
\hline Networks & \multicolumn{3}{|c|}{ WLAN } & \multicolumn{3}{c|}{ WMAN } & \multicolumn{3}{c|}{ WWAN } \\
\hline Traffic Class & $d\left(A_{i}, A^{+}\right)$ & $d\left(A_{i}, A^{-}\right)$ & $C C$ & $d\left(A_{i}, A^{+}\right)$ & $d\left(A_{i}, A^{-}\right)$ & $C C$ & $d\left(A_{i}, A^{+}\right)$ & $d\left(A_{i}, A^{-}\right)$ & $C C$ \\
\hline Streaming & 1.9254 & 0.7151 & 0.2708 & 0.7230 & 1.9217 & 0.7266 & 1.3656 & 1.2886 & 0.4855 \\
\hline Conversational & 2.208 & 0.5619 & 0.2029 & 0.7230 & 2.0544 & 0.7397 & 1.0674 & 1.7072 & 0.6133 \\
\hline Interactive & 1.8193 & 0.7151 & 0.2821 & 0.6536 & 1.8828 & 0.7423 & 1.2644 & 1.2837 & 0.5038 \\
\hline Background & 1.6726 & 0.5619 & 0.2515 & 0.5004 & 1.7362 & 0.7763 & 1.1491 & 1.0991 & 0.4889 \\
\hline
\end{tabular}


Table 4.9: FVIKOR Decision Matrix, showing FBV $\left(\tilde{f}_{j}^{+}\right)$and FWV $\left(\tilde{f}_{j}^{-}\right)$, Streaming Traffic

\begin{tabular}{|c|c|c|c|c|c|c|c|c|c|c|}
\hline Networks & RSS & Delay & Jitter & PLR & T.put & Velocity & Loading & Security & Cost \\
\hline WLAN & {$[0.2,0.4,0.6]$} & {$[0.4,0.6,0.8]$} & {$[0.8,1.0,1.0]$} & {$[0.0,0.2,0.4]$} & {$[0.2,0.4,0.6]$} & {$[0.8,1.0,1.0]$} & {$[0.0,0.2,0.4]$} & {$[0.0,0.2,0.4]$} & {$[0.0,0.2,0.4]$} \\
\hline WMAN & {$[0.4,0.6,0.8]$} & {$[0.0,0.2,0.4]$} & {$[0.4,0.6,0.8]$} & {$[0.4,0.6,0.8]$} & {$[0.6,0.8,1.0]$} & {$[0.0,0.2,0.4]$} & {$[0.0,0.2,0.4]$} & {$[0.2,0.4,0.6]$} & {$[0.2,0.4,0.6]$} \\
\hline WWAN & {$[0.4,0.6,0.8]$} & {$[0.0,0.2,0.4]$} & {$[0.0,0.2,0.4]$} & {$[0.4,0.6,0.8]$} & {$[0.4,0.6,0.8]$} & {$[0.4,0.6,0.8]$} & {$[0.2,0.4,0.6]$} & {$[0.6,0.8,1.0]$} & {$[0.6,0.8,1.0]$} \\
\hline FBV $\left(\tilde{f}_{j}^{+}\right)$ & {$[0.4,0.6,0.8]$} & {$[0.0,0.2,0.4]$} & {$[0.0,0.2,0.4]$} & {$[0.0,0.2,0.4]$} & {$[0.6,0.8,1.0]$} & {$[0.0,0.2,0.4]$} & {$[0.0,0.2,0.4]$} & {$[0.6,0.8,1.0]$} & {$[0.0,0.2,0.4]$} \\
\hline FWV $\left(\tilde{f}_{j}^{-}\right)$ & {$[0.2,0.4,0.6]$} & {$[0.4,0.6,0.8]$} & {$[0.8,1.0,1.0]$} & {$[0.4,0.6,0.8]$} & {$[0.2,0.4,0.6]$} & {$[0.8,1.0,1.0]$} & {$[0.2,0.4,0.6]$} & {$[0.0,0.2,0.4]$} & {$[0.6,0.8,1.0]$} \\
\hline
\end{tabular}


Table 4.10: FVIKOR Calculations for All Traffic Classes

\begin{tabular}{|c|c|c|c|c|c|c|c|c|c|c|c|c|c|}
\hline \multirow[t]{2}{*}{ Traffic Classes } & \multicolumn{4}{|c|}{ Indices } & \multicolumn{3}{|c|}{ WLAN } & \multicolumn{3}{|c|}{ WMAN } & \multicolumn{3}{|c|}{ WWAN } \\
\hline & $S^{+}$ & $R^{+}$ & $S^{-}$ & $R^{-}$ & $S_{i}$ & $R_{i}$ & $Q_{i}$ & $S_{i}$ & $R_{i}$ & $Q_{i}$ & $S_{i}$ & $R_{i}$ & $Q_{i}$ \\
\hline Streaming & $\begin{array}{c}{[-7.00} \\
1.80 \\
3.50]\end{array}$ & $\begin{array}{c}{[0.40} \\
0.80 \\
4.00]\end{array}$ & $\begin{array}{c}{[-1.80} \\
3.40 \\
7.90]\end{array}$ & $\begin{array}{c}{[0.99} \\
1.00 \\
8.00]\end{array}$ & $\begin{array}{c}{[-1.80} \\
3.40 \\
7.90]\end{array}$ & $\begin{array}{l}{[0.99,} \\
1.00, \\
8.00]\end{array}$ & $\begin{array}{c}{[-0.40,} \\
1.00 \\
-2.70]\end{array}$ & $\begin{array}{c}{[-5.90} \\
1.80 \\
3.50]\end{array}$ & $\begin{array}{l}{[0.40,} \\
0.80, \\
4.00]\end{array}$ & $\begin{array}{c}{[-0.55} \\
0.28 \\
-1.59]\end{array}$ & $\begin{array}{c}{[-7.00} \\
3.10 \\
4.80]\end{array}$ & $\begin{array}{c}{[0.79,} \\
0.80, \\
6.00]\end{array}$ & $\begin{array}{c}{[-0.56,} \\
0.64, \\
-2.04]\end{array}$ \\
\hline Conversational & $\begin{array}{c}{[-6.33} \\
1.20 \\
0.78]\end{array}$ & $\begin{array}{l}{[0.80,} \\
0.50 \\
2.40]\end{array}$ & $\begin{array}{c}{[-4.64} \\
3.91 \\
3.35]\end{array}$ & $\begin{array}{c}{[1.49} \\
1.00 \\
3.20\end{array}$ & $\begin{array}{c}{[-5.66,} \\
3.91 \\
3.35]\end{array}$ & $\begin{array}{l}{[1.49,} \\
1.00, \\
3.20]\end{array}$ & $\begin{array}{c}{[-0.52} \\
1.00 \\
-2.22]\end{array}$ & $\begin{array}{c}{[-4.64} \\
1.20 \\
0.79]\end{array}$ & $\begin{array}{l}{[1.20,} \\
0.50, \\
2.40]\end{array}$ & $\begin{array}{c}{[-0.53} \\
0.12 \\
-1.54]\end{array}$ & $\begin{array}{c}{[-6.33} \\
2.00 \\
0.78]\end{array}$ & $\begin{array}{l}{[0.80,} \\
0.60, \\
2.40]\end{array}$ & $\begin{array}{c}{[-0.70} \\
0.25 \\
-1.54]\end{array}$ \\
\hline Interactive & $\begin{array}{c}{[-9.36} \\
1.40 \\
1.60]\end{array}$ & $\begin{array}{c}{[0.19} \\
0.80 \\
2.4]\end{array}$ & $\begin{array}{c}{[-7.10} \\
3.22 \\
4.51]\end{array}$ & $\begin{array}{c}{[0.99} \\
1.00 \\
4.80]\end{array}$ & $\begin{array}{c}{[-7.10} \\
3.22 \\
4.51]\end{array}$ & $\begin{array}{l}{[0.99,} \\
1.00, \\
4.80]\end{array}$ & $\begin{array}{c}{[-0.47,} \\
1.00 \\
-2.43]\end{array}$ & $\begin{array}{c}{[-8.31} \\
1.40 \\
1.60]\end{array}$ & $\begin{array}{l}{[0.19,} \\
0.80, \\
2.40]\end{array}$ & $\begin{array}{c}{[-0.60} \\
0.28, \\
-1.41]\end{array}$ & $\begin{array}{c}{[-9.36} \\
2.62 \\
2.17]\end{array}$ & $\begin{array}{l}{[0.79,} \\
0.80, \\
3.60]\end{array}$ & $\begin{array}{c}{[-0.57} \\
0.59 \\
-1.87]\end{array}$ \\
\hline Background & $\begin{array}{c}{[-3.88} \\
1.20 \\
2.75]\end{array}$ & $\begin{array}{c}{[0.19} \\
0.50 \\
3.2]\end{array}$ & $\begin{array}{c}{[-0.77} \\
2.91 \\
6.38]\end{array}$ & $\begin{array}{c}{[0.99} \\
1.00 \\
6.40]\end{array}$ & $\begin{array}{c}{[-0.77} \\
2.92 \\
6.38]\end{array}$ & $\begin{array}{l}{[0.99,} \\
1.00, \\
6.40]\end{array}$ & $\begin{array}{c}{[-0.35} \\
1.00 \\
-2.87]\end{array}$ & $\begin{array}{c}{[-2.88,} \\
1.20 \\
2.75]\end{array}$ & $\begin{array}{l}{[0.19,} \\
0.50, \\
3.20]\end{array}$ & $\begin{array}{c}{[-0.52,} \\
0.13 \\
-1.62]\end{array}$ & $\begin{array}{c}{[-3.88} \\
2.47 \\
3.71]\end{array}$ & $\begin{array}{l}{[0.79,} \\
0.60, \\
4.80]\end{array}$ & $\begin{array}{c}{[-0.52,} \\
0.47 \\
-2.12]\end{array}$ \\
\hline
\end{tabular}


The system model is shown in Figure 4.20. The straight-line path of a single MS crossing several cell boundaries can be seen from Figure 4.21, where different wireless access technologies are shown providing coverage to the moving MS.

Mobility Model: The mobility model is created to simulate the mobility of MS within each cell. To simulate VHITS, a mobility model is implemented where MSs are uniformly distributed over the entire system. Each MS moves a random distance at defined time steps. The mobility pattern of each MS depends on its velocity and acceleration. The velocity $V_{j}$ of the $j^{\text {th }}$ MS is updated using Equation (4.1).

$$
V_{j}=\left(V_{j-1} \times P\right)+\left(\widetilde{V} \times R \times \sqrt{1-P^{2}}\right)
$$

where the updated velocity of the MS is given by $V_{j}, V_{j-1}$ is the velocity of the mobile user in the previous time-step, and $\widetilde{V}$ is the mean velocity of MS. The variable $R$ is a Rayleigh distributed magnitude with a mean value of 1 and the variable $P$ is used to correlate the velocity between simulated time-steps and is based on both mean velocity and mean acceleration. The value of $P$ can be calculated from Equation (4.2) as follows:

$$
P=e^{\frac{-\Delta t \times \widetilde{a}}{\tilde{V}}}
$$

where $\tilde{a}$ is the mean acceleration of the MS.

Service Model: The service model is implemented to specify the four types of services, each with unique characteristics. Chapter 3 explains the requirements of Streaming, Conversational, Background, and Interactive traffic classes considered in this study. Most of the simulations performed are based on these traffic classes to study the behavior of available networks with varying conditions. 
Traffic Model: The generation of MSs for the simulation is done with the help of traffic model. All MSs utilizing the system are created using the Poisson process [138] to make sure that the arrivals of new calls and their subsequent departures are exponentially distributed. These generated MSs are distributed uniformly among all the cells using an average Poisson arrival rate. This is shown in Figure 4.22.

Propagation Model: The propagation model is the most important model, as the performance of any wireless communication system depends on how well the radio waves propagate through the medium. These radio waves, propagated via radio channels are affected by phenomenon like reflection, scattering, and diffraction. Hence, a mobility model is developed that considers different losses and gains during the signal propagation between the MS and the BS/AP. The radio propagation model that is used in this simulation work considers path losses due to signal attenuation based on distance, antenna gain, and both shadow and Rayleigh fading. This model can be defined as the logarithmic sum of all of these components. This is given in Equation (4.3).

$$
G=G_{\text {Path-Loss }}+G_{\text {Antenna }}+G_{\text {Shadow }}+G_{\text {Rayleigh }}
$$

As the distance between the MS and the BS/AP increases, the wireless signal attenuates. This signal attenuation is modeled using Hata Path Loss model [139] and is given in Equation (4.4).

$$
G_{\text {Path-Loss }}=10 \times \eta \times \log _{10} d+C
$$

where $d$ is the distance between the transmitter and the receiver, $\eta$ is the path loss exponent that relates the transmitted-power decay with distance, and $C$ is a constant accounting for different system losses depending on carrier frequency, the size of the antenna, and other physical parameters. 


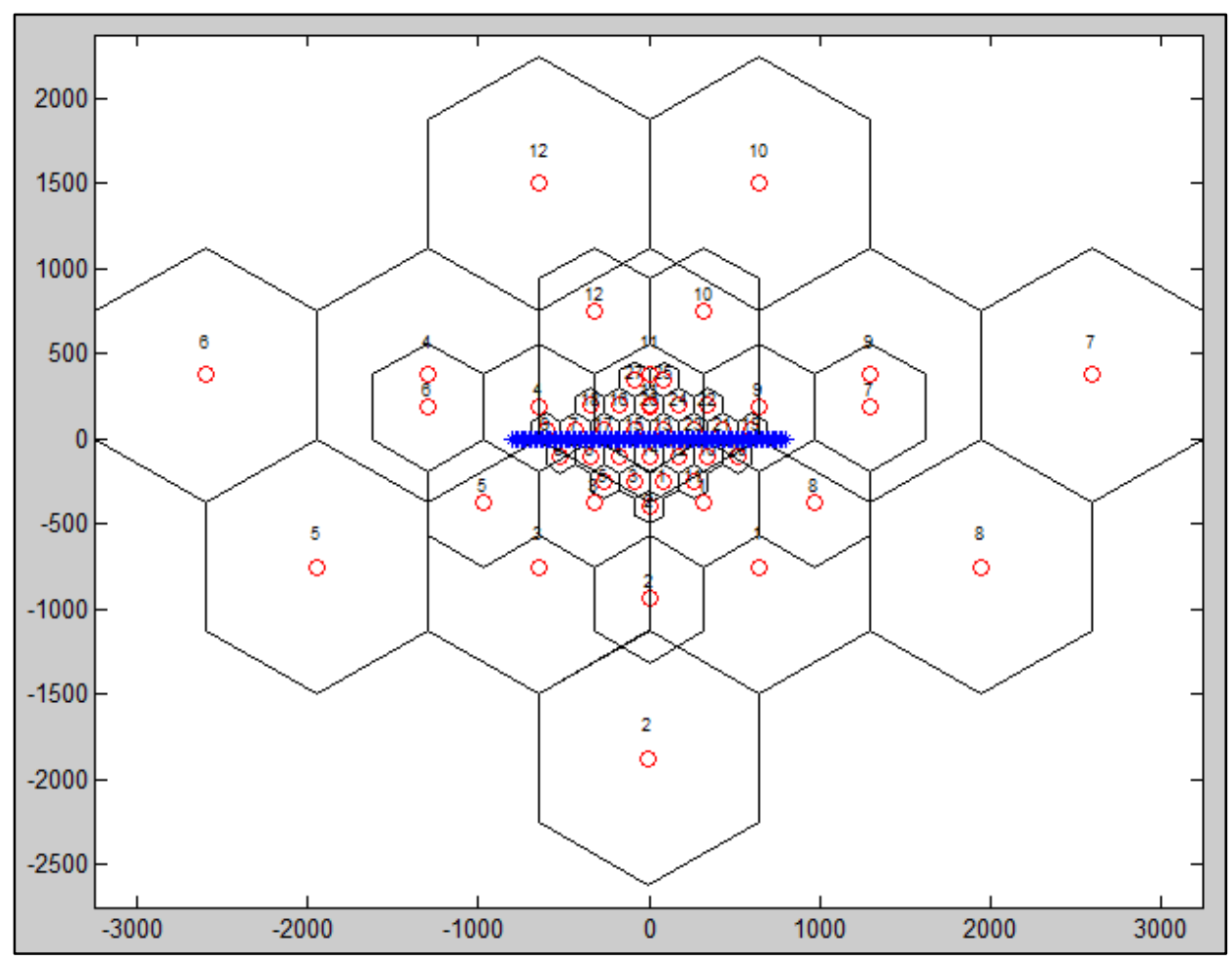

Figure 4.20: System Model for VHITS Simulation

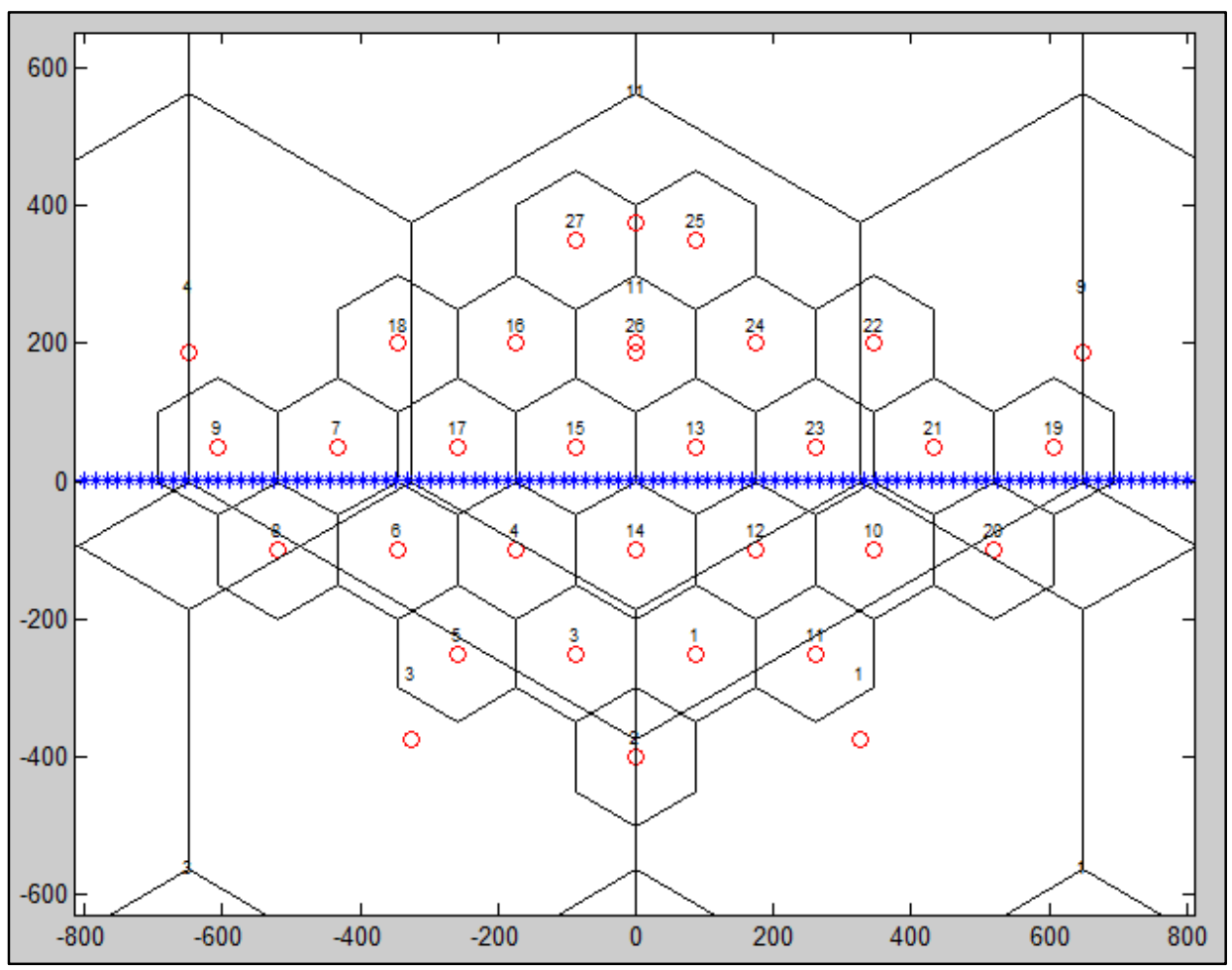

Figure 4.21: System model (zoomed-in) Depicting the Mobility of Single User 


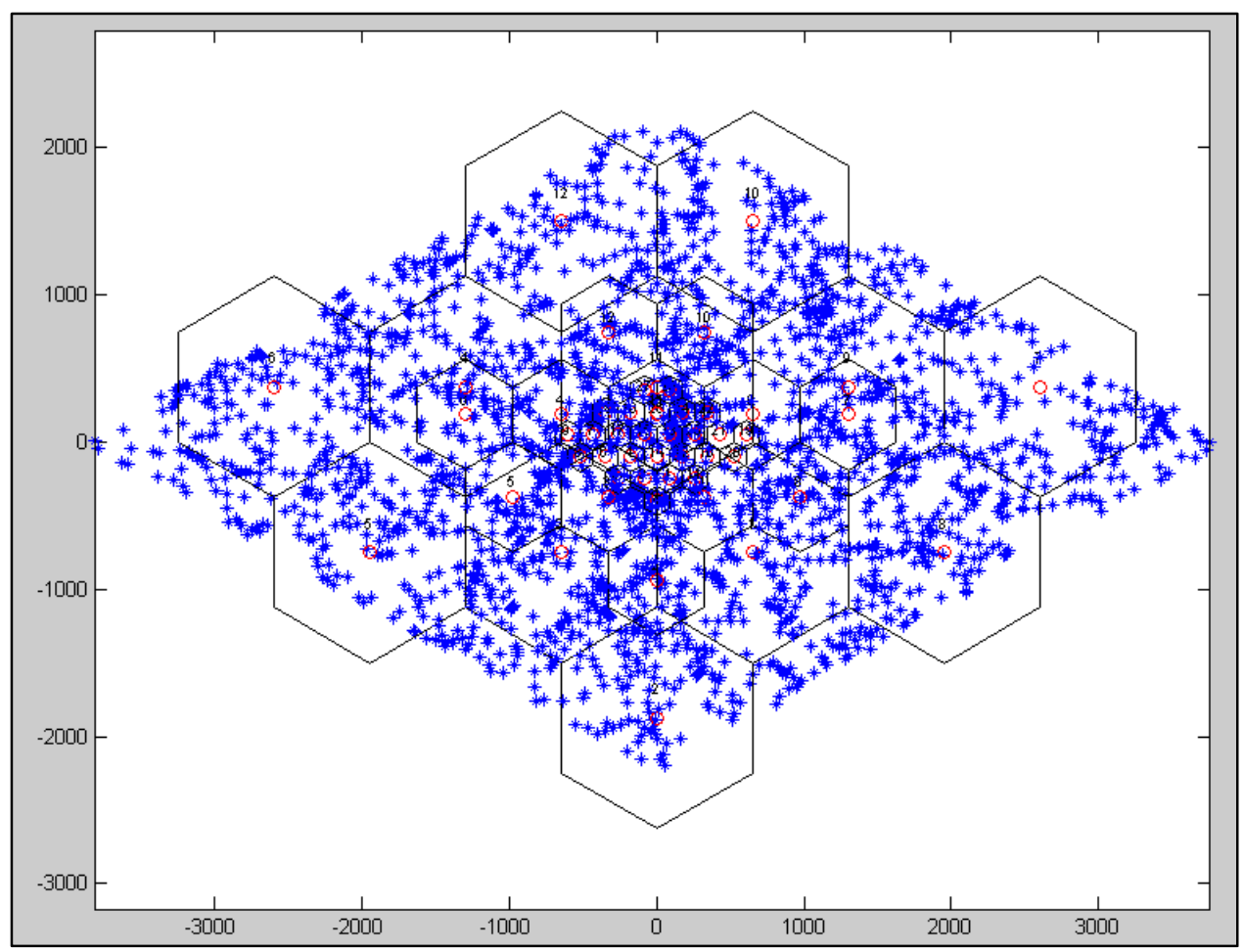

Figure 4.22: Distribution of MSs across Different Networks

In wireless communication, fading [140] is defined as the rapid fluctuations of the signal that it experiences over certain propagation media. There are two types of fading: Shadow Fading and Rayleigh Fading. Shadow fading is used to statistically model the effect of signal attenuation due to large object, such as a building located between the transmitter and the receiver. In this type of fading, the amplitude and phase change imposed by the channel can be considered roughly constant over the period of use. This change in the amplitude is often modeled using a log-normal distribution with a standard deviation according to the log-distance path loss model. On the other hand, Rayleigh fading is used to model the effect of scattering due to the presence of different objects between the transmitter and the receiver resulting in multi-path propagation of signal with 
no line-of-sight between the two end-stations. In this case, the change in amplitude and phase varies considerably over the period of use.

Different network parameters that are used for simulating the proposed scheme are depicted in Table 4.11. These parameters are randomly selected to indicate the QoS, security offered by a specific network, and their usage cost. The values for RSS and Network-Loading are obtained from the test-bed as they are different for each iteration, where MS-Velocity varying from $0-10 \mathrm{~m} / \mathrm{s}$. The maximum velocity of $10 \mathrm{~m} / \mathrm{s}$ is selected to match the WLAN cell radius of $100 \mathrm{~m}$.

Table 4.11: Network Parameters used for VHITS Simulation

\begin{tabular}{|c|c|c|c|c|c|c|}
\hline & $\begin{array}{c}\text { Delay } \\
(\mathbf{m s})\end{array}$ & $\begin{array}{c}\text { Jitter } \\
(\mathbf{m s})\end{array}$ & $\begin{array}{c}\text { PLR } \\
\text { (per } \mathbf{1 0}^{\mathbf{6}}\end{array}$ & $\begin{array}{c}\text { Throughput } \\
\text { bytes) }\end{array}$ & $\begin{array}{c}\text { Security } \\
\text { (Mlops) }\end{array}$ & $\begin{array}{c}\text { Cost } \\
(\mathbf{1 - 1 0})\end{array}$ \\
\hline WLAN & 130 & 30 & 5 & 140 & 5 & 2 \\
\hline WMAN & 30 & 10 & 4 & 50 & 5 & 4 \\
\hline WWAN & 10 & 1 & 2 & 0.2 & 5 & 7 \\
\hline
\end{tabular}

Table 4.12 summarizes some of the system parameters to setup the simulation environment.

\subsection{SIMULATION RESULTS: SINGLE MOBILE USER}

In this section, we present different simulation results assuming the presence of only one MS moving in a straight line with access to different wireless networks. The percentage of network connection is a metric that is used to evaluate the performance of our scheme. This metric indicates the percentages of networks' connections that our proposed scheme prefers for an MS moving with a speed of $0-10 \mathrm{~m} / \mathrm{s}$. Note that this percentage is calculated after the system completes the entire simulation. 
Table 4.12: System Parameters used for VHITS Simulation

\begin{tabular}{|c|c|c|c|}
\hline Parameters & WLAN & WMAN & WWAN \\
\hline Cell Shape & \multicolumn{3}{|c|}{ Hexagonal with Omni-directional Antennas } \\
\hline Cell Size (meters) & 100 & 375 & 750 \\
\hline Number of Cells & 27 & 12 & 12 \\
\hline Number of Clusters & 9 & 4 & 4 \\
\hline Allocated Channels per Cell & 8 & 12 & 16 \\
\hline Mean Velocity $(\mathrm{m} / \mathrm{s})$ & \multicolumn{3}{|c|}{$0-10$} \\
\hline Mean Acceleration $\left(\mathrm{m} / \mathrm{s}^{2}\right)$ & \multicolumn{3}{|c|}{0.01} \\
\hline Mean Service holding time (sec) & \multicolumn{3}{|c|}{20} \\
\hline Thermal Noise Floor (dbm) & \multicolumn{3}{|c|}{-118} \\
\hline Standard deviation for fading (dB) & \multicolumn{3}{|c|}{6} \\
\hline Fading Correlation (downlink) & \multicolumn{3}{|c|}{0.5} \\
\hline Fading Correlation distance (m) & \multicolumn{3}{|c|}{20} \\
\hline Average Number of Calls per Cell & \multicolumn{3}{|c|}{$1-10$} \\
\hline Min. RSS to connect (dbm) & -110 & -160 & -150 \\
\hline Attenuation at $1 \mathrm{~m}$ distance (dB) & -40 & -55 & -28 \\
\hline Path Loss Exponent & 3.3 & 4 & 4 \\
\hline
\end{tabular}

\subsubsection{TOPSIS BASED NETWORK SELECTION}

This section provides the results for the network selection based on TOPSIS ranking algorithm with AHP weighting method. Figures 4.23-4.26 show the percentages of connections to different types of wireless networks that the MS prefers for any of the four traffic classes.

Based on the network parameters provided in Table 4.11, the proposed scheme for the Conversational traffic class prefers to connect to WLAN approximately $98 \%$ of the 
time, for the slower moving MS. As depicted in Figure 4.23, at medium speed, WMAN can be seen as the preferred network with approximately $61 \%$ connections. Similarly, for a higher speed MS, the preferred connectivity is to WWAN. It can be noted that WLAN shows a strong presence at medium and high speeds with higher preference towards WLAN as compared with other networks with MS-speeds of 6-7 m/s.

A similar pattern like Conversational traffic can be observed for Interactive traffic class from Figure 4.24. At an MS speed of $7 \mathrm{~m} / \mathrm{s}$, a high connectivity preference of approximately $90 \%$ can be seen towards WLAN.

For Background traffic class, WLAN seems to be the preferred network for slow and fast moving MS. It can be observed from Figure 4.25 that an MS moving at slower or higher speed, WLAN connectivity preference is approximately 90\%-95\%. A similar behavior can be observed for Streaming traffic class, as shown in Figure 4.26.

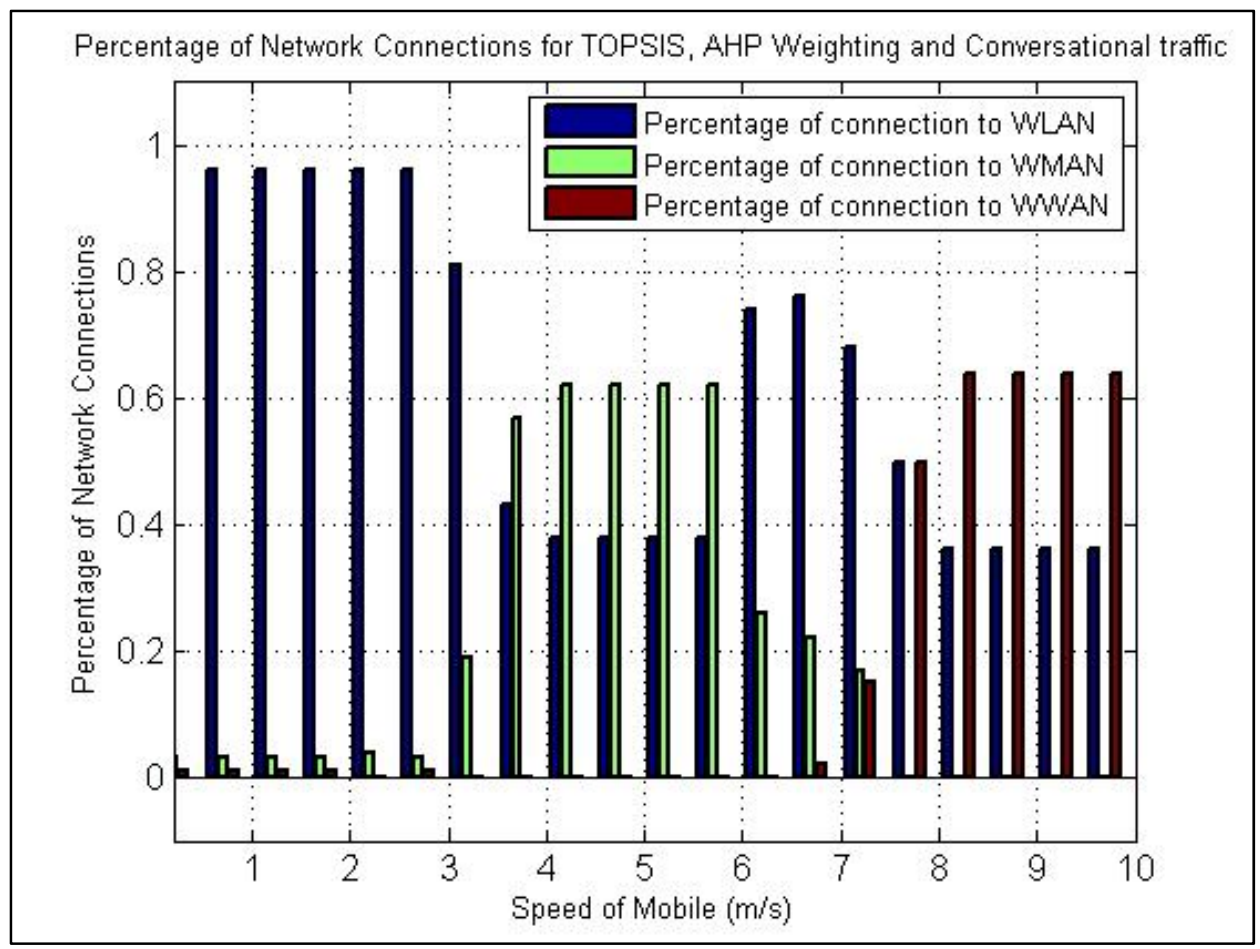

Figure 4.23: Percentage of NW-Connection, TOPSIS, AHP, for Conversational 


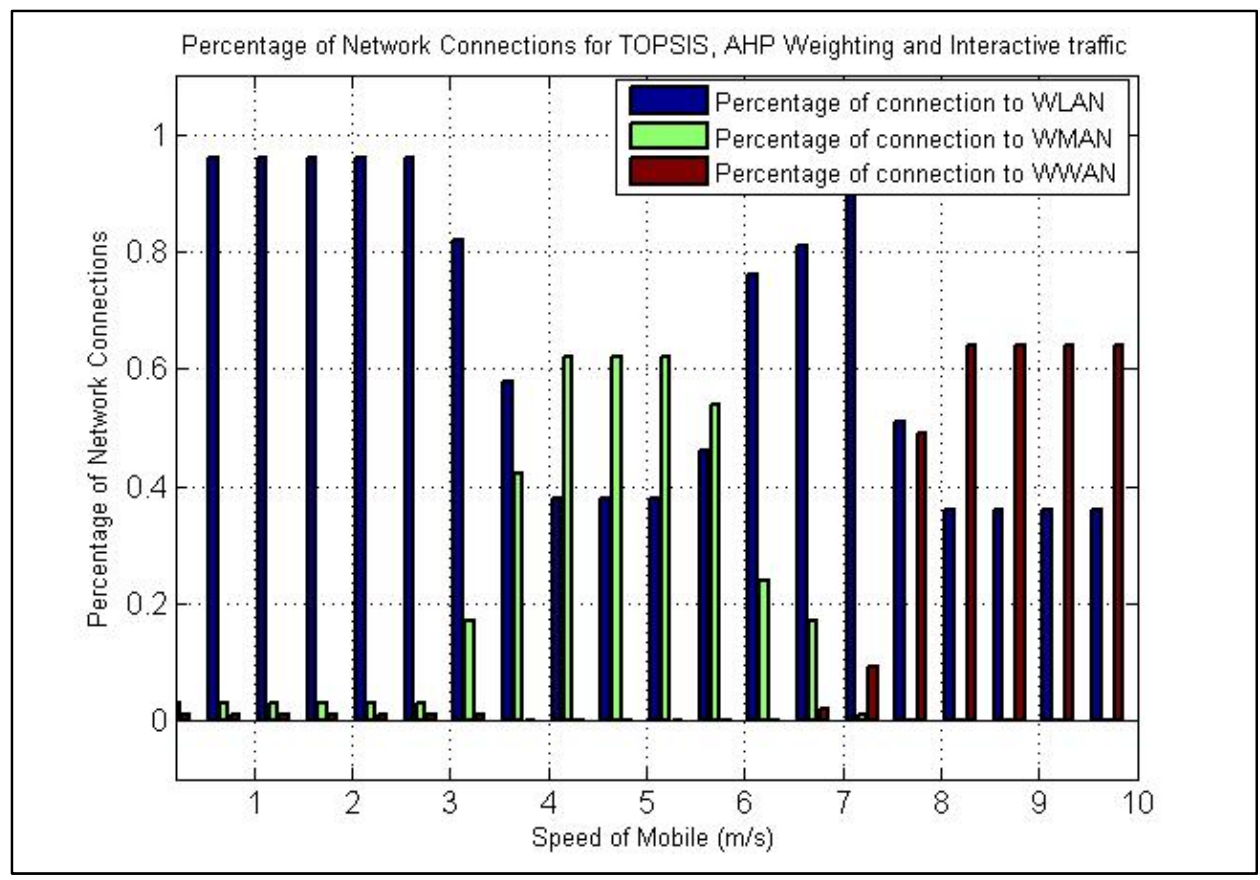

Figure 4.24: Percentage of NW-Connection, TOPSIS, AHP, for Interactive

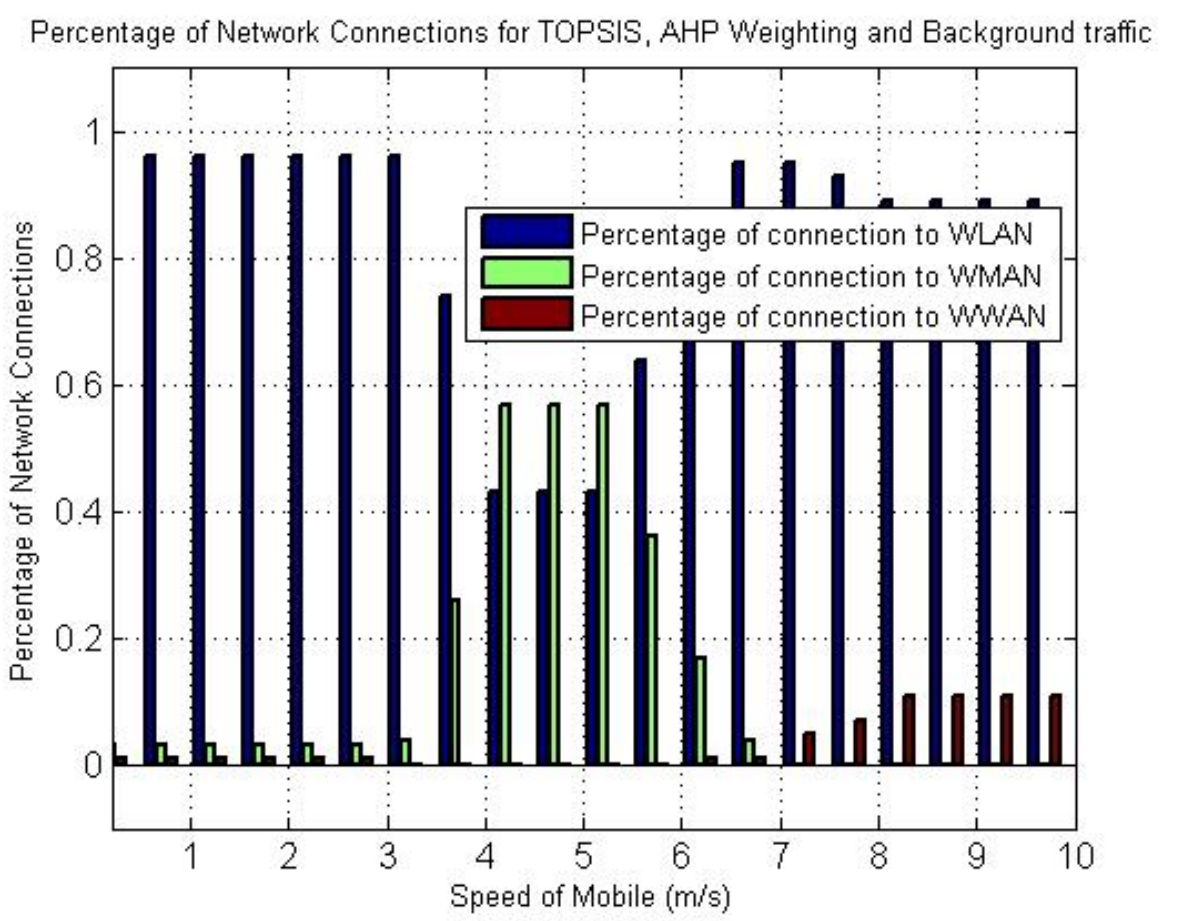

Figure 4.25: Percentage of NW-Connection, TOPSIS, AHP, for Background 


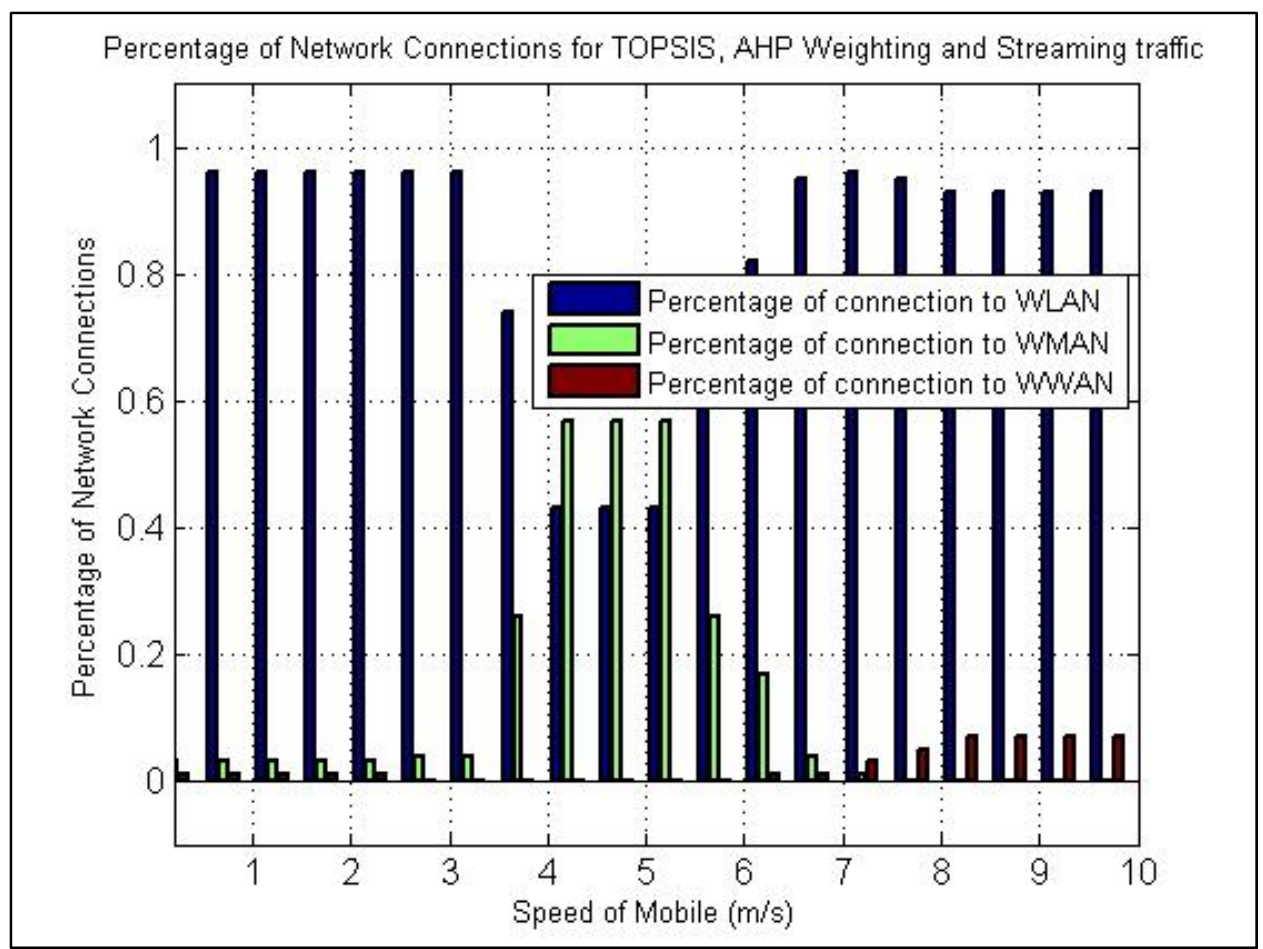

Figure 4.26: Percentage of NW-Connection, TOPSIS, AHP, for Streaming

Network selection based on TOPSIS and FAHP weighting schemes is presented in Figures 4.27-4.30, whereas Figures 4.31-4.34 show simulation results from the same ranking algorithm, but using TFN weighting scheme. As explained earlier, both FAHP and TFN schemes are based on fuzzy data to incorporate the vagueness and uncertainty inherent in the selection of priority weights for network attributes. Both FAHP and TFN weighting schemes, when used with TOPSIS ranking algorithm, show a clear choice of network connectivity preferences at slower, medium and higher speeds. 


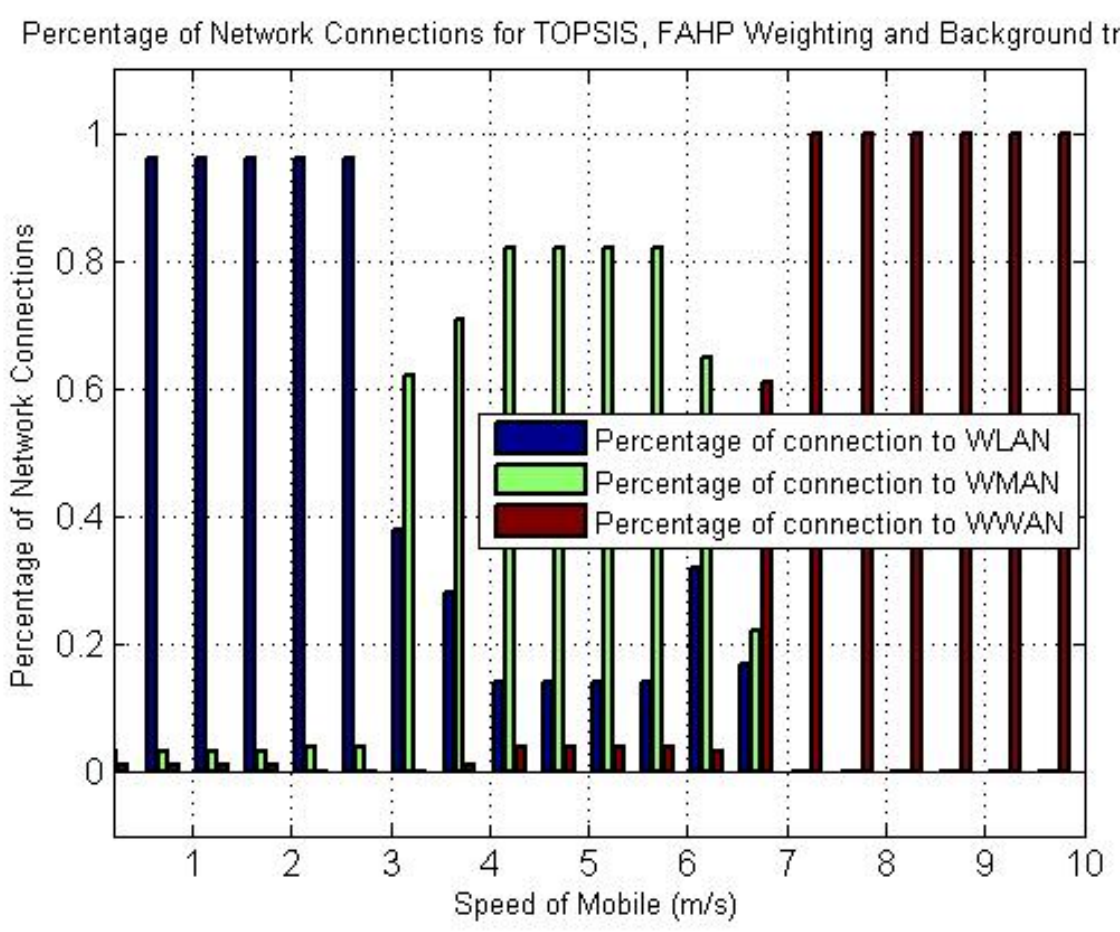

Figure 4.27: Percentage of NW-Connection, TOPSIS, FAHP, for Background

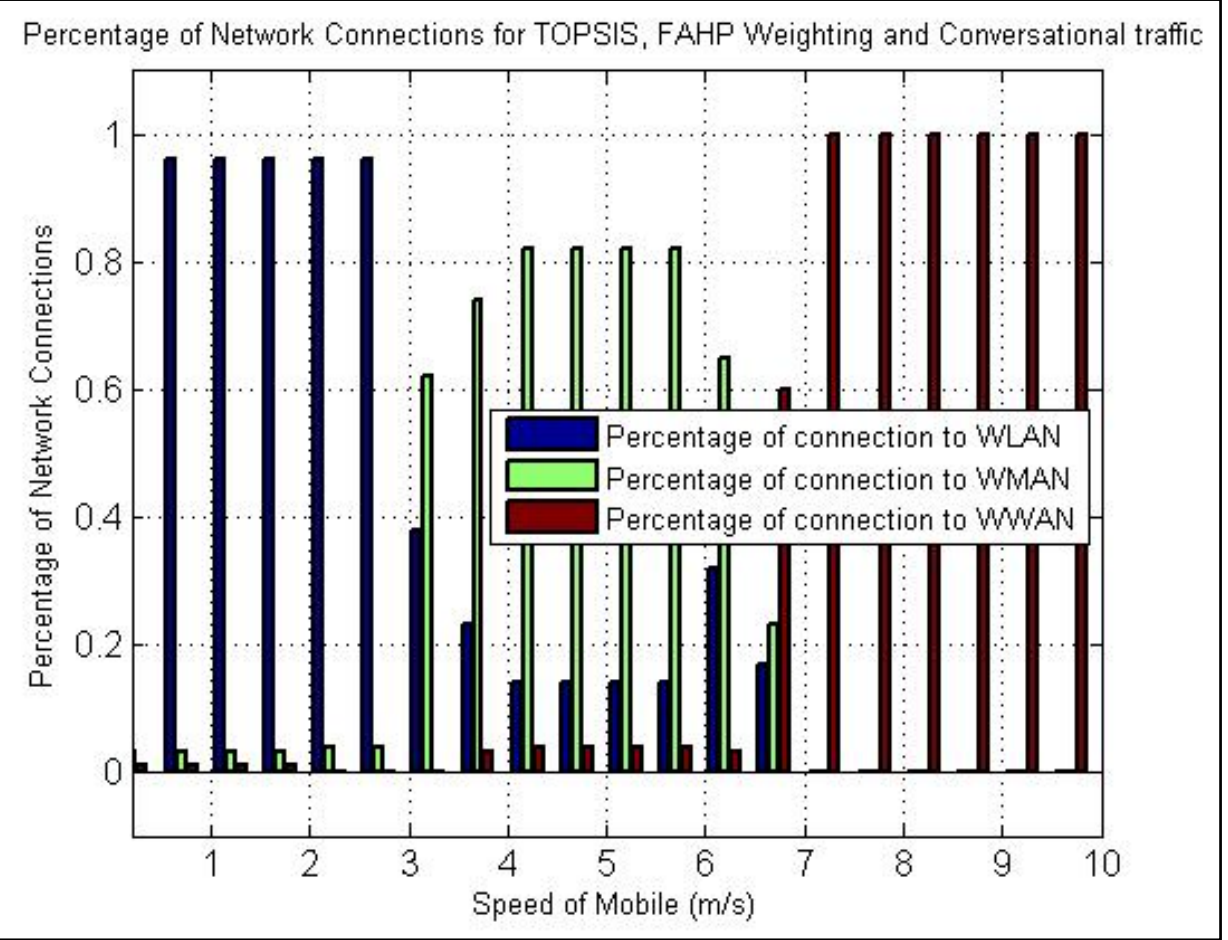

Figure 4.28: Percentage of NW-Connection, TOPSIS, FAHP, for Conversational 


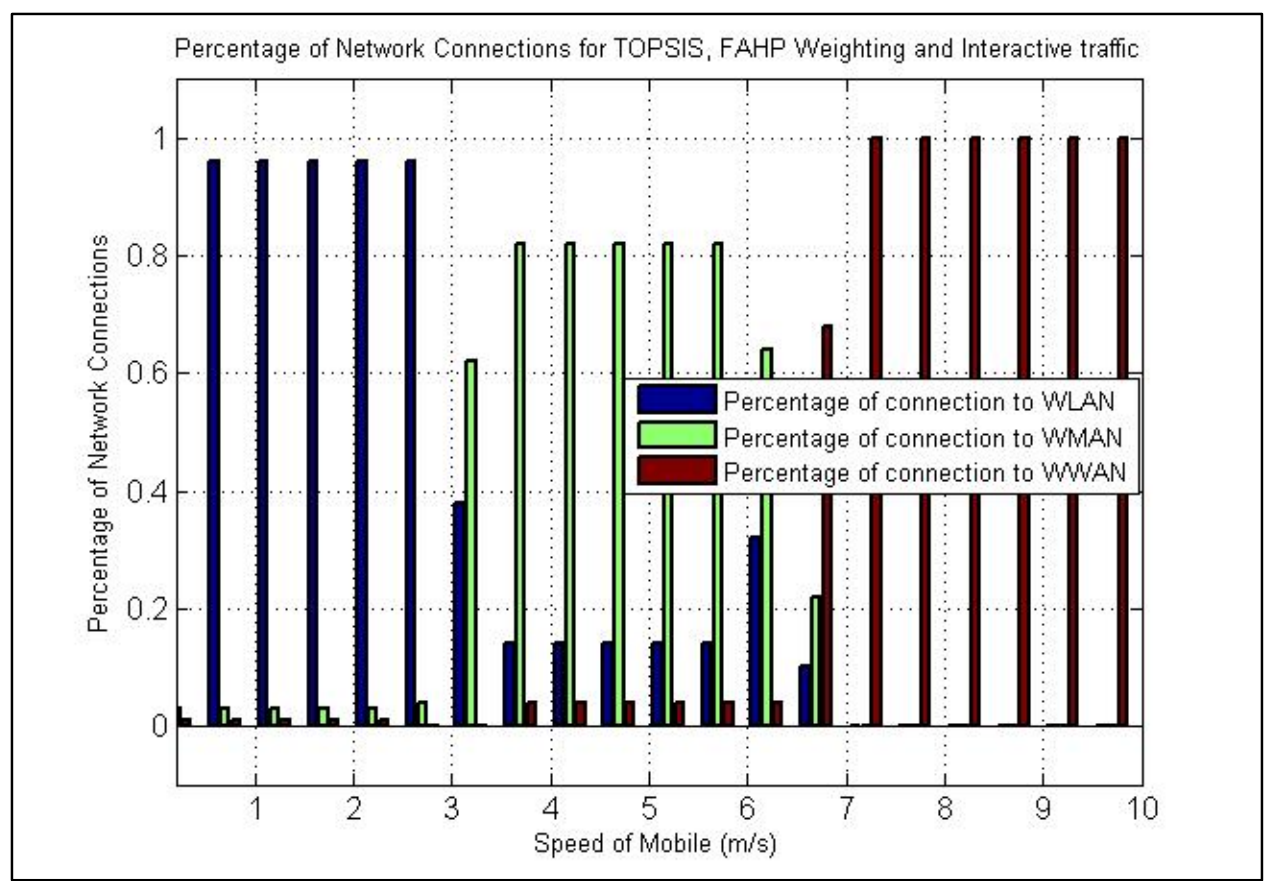

Figure 4.29: Percentage of NW-Connection, TOPSIS, FAHP, for Interactive

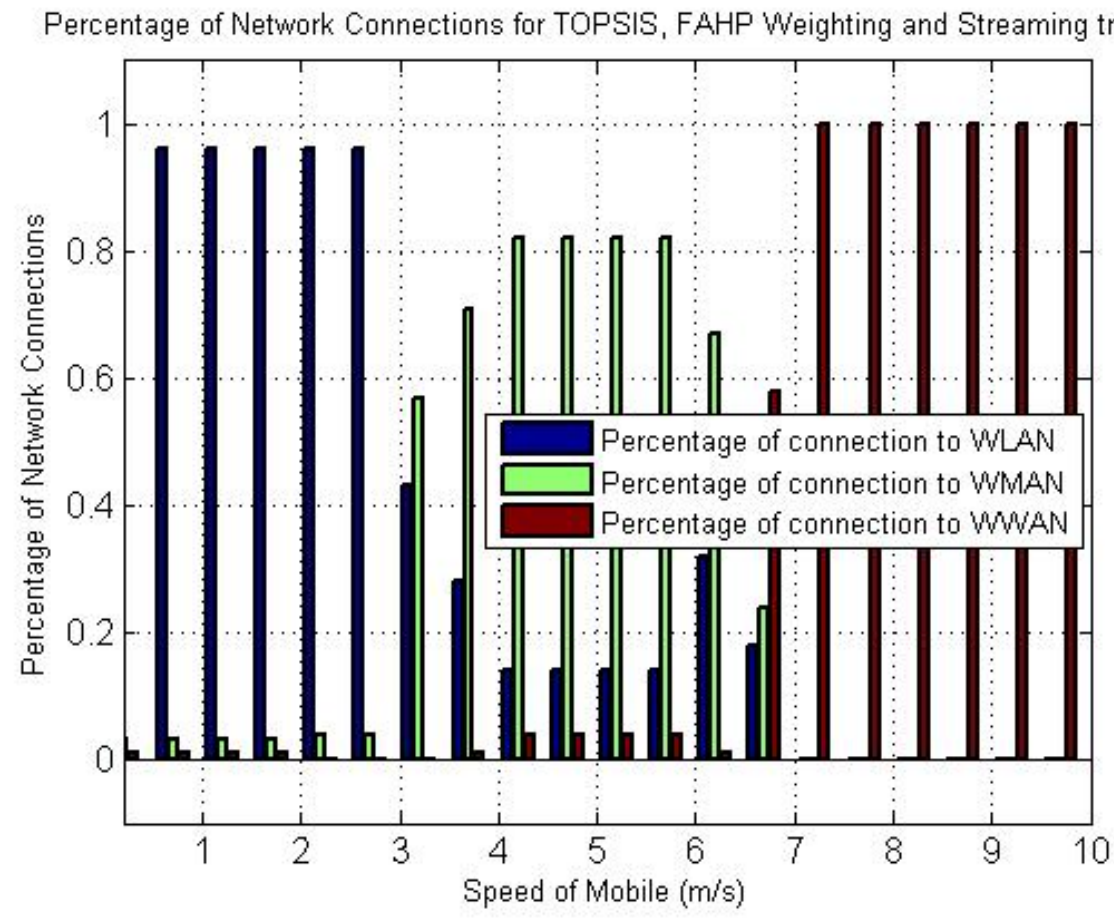

Figure 4.30: Percentage of NW-Connection, TOPSIS, FAHP, for Streaming 


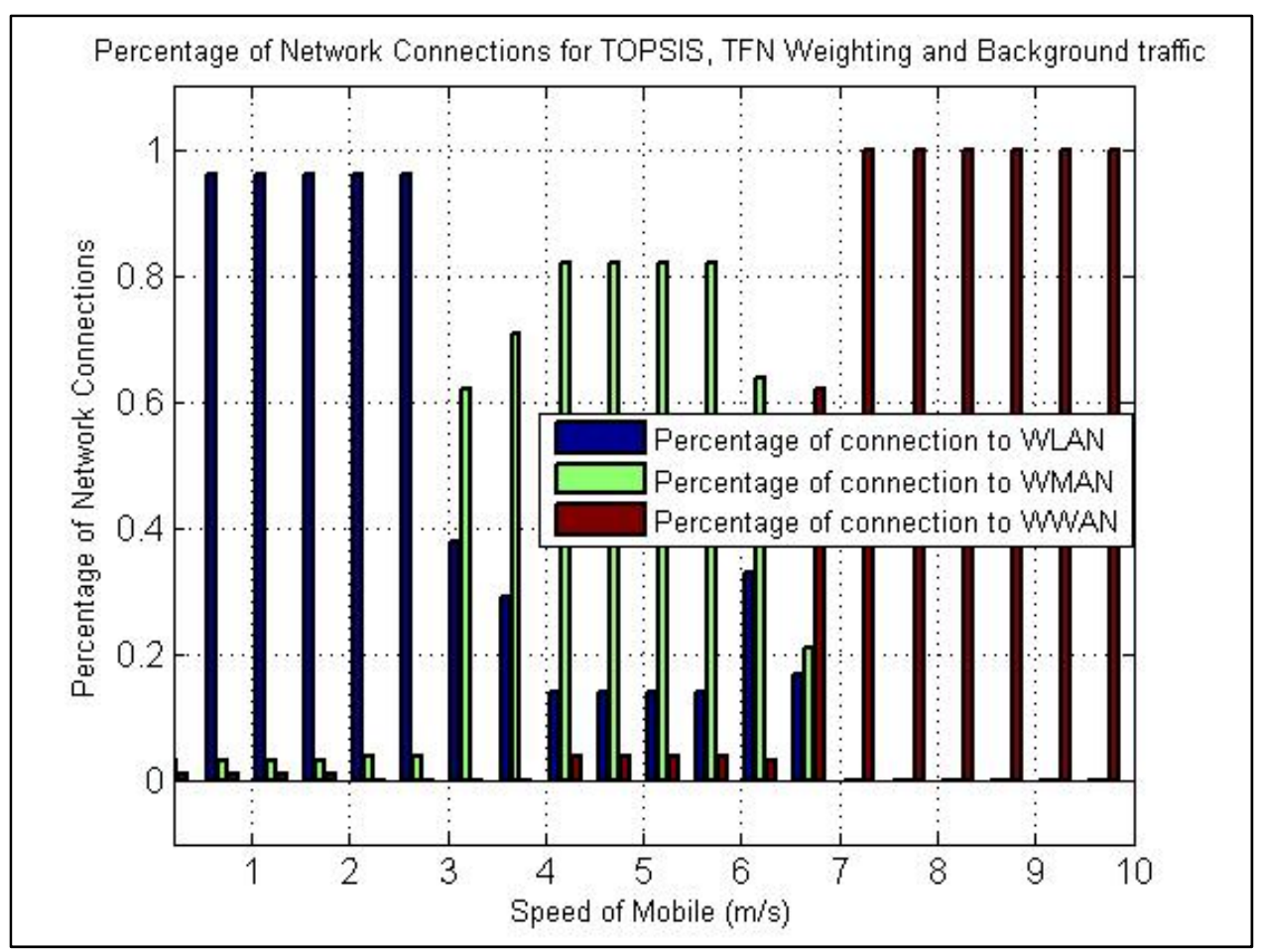

Figure 4.31: Percentage of NW-Connection, TOPSIS, TFN, for Background

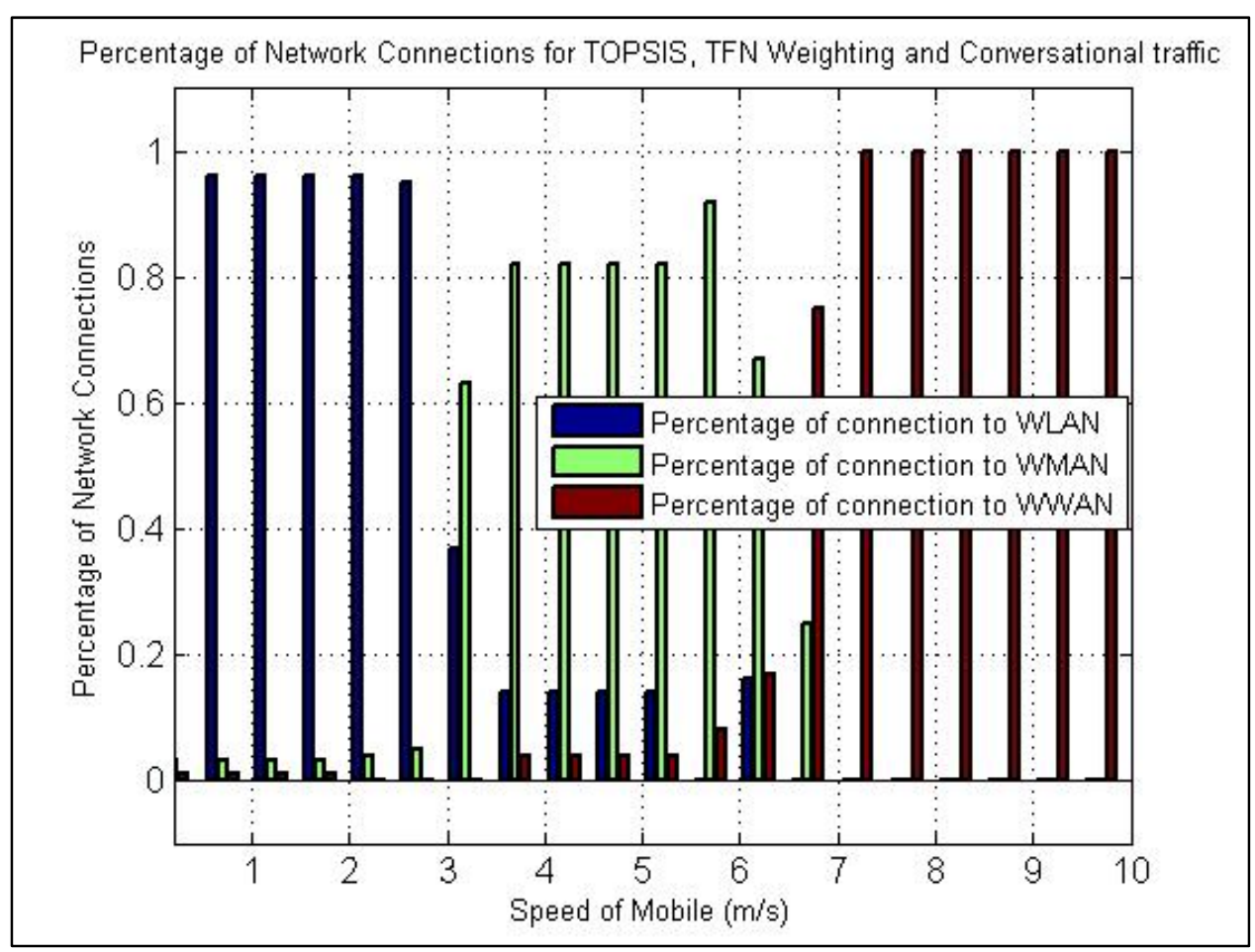

Figure 4.32: Percentage of NW-Connection, TOPSIS, TFN, for Conversational 


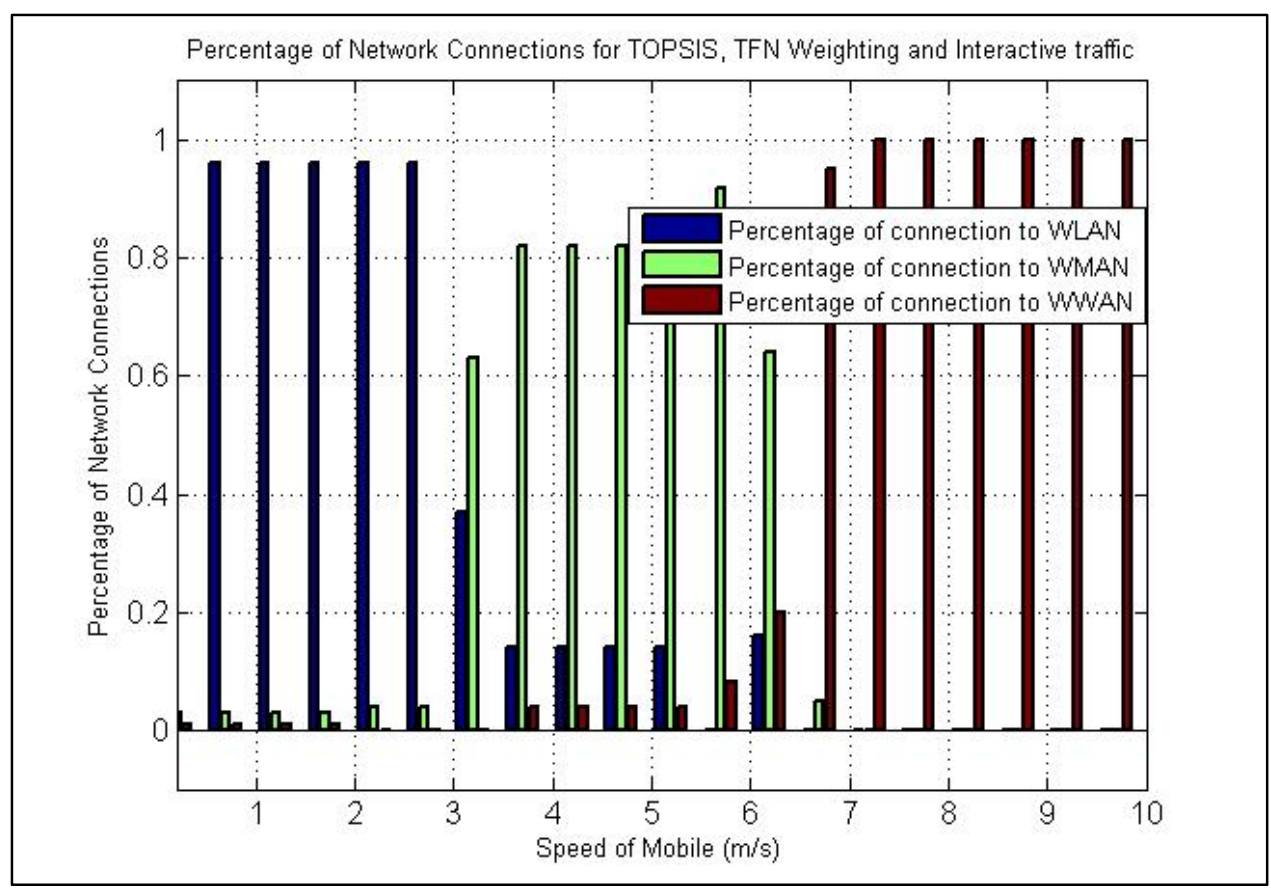

Figure 4.33: Percentage of NW-Connection, TOPSIS, TFN, for Interactive

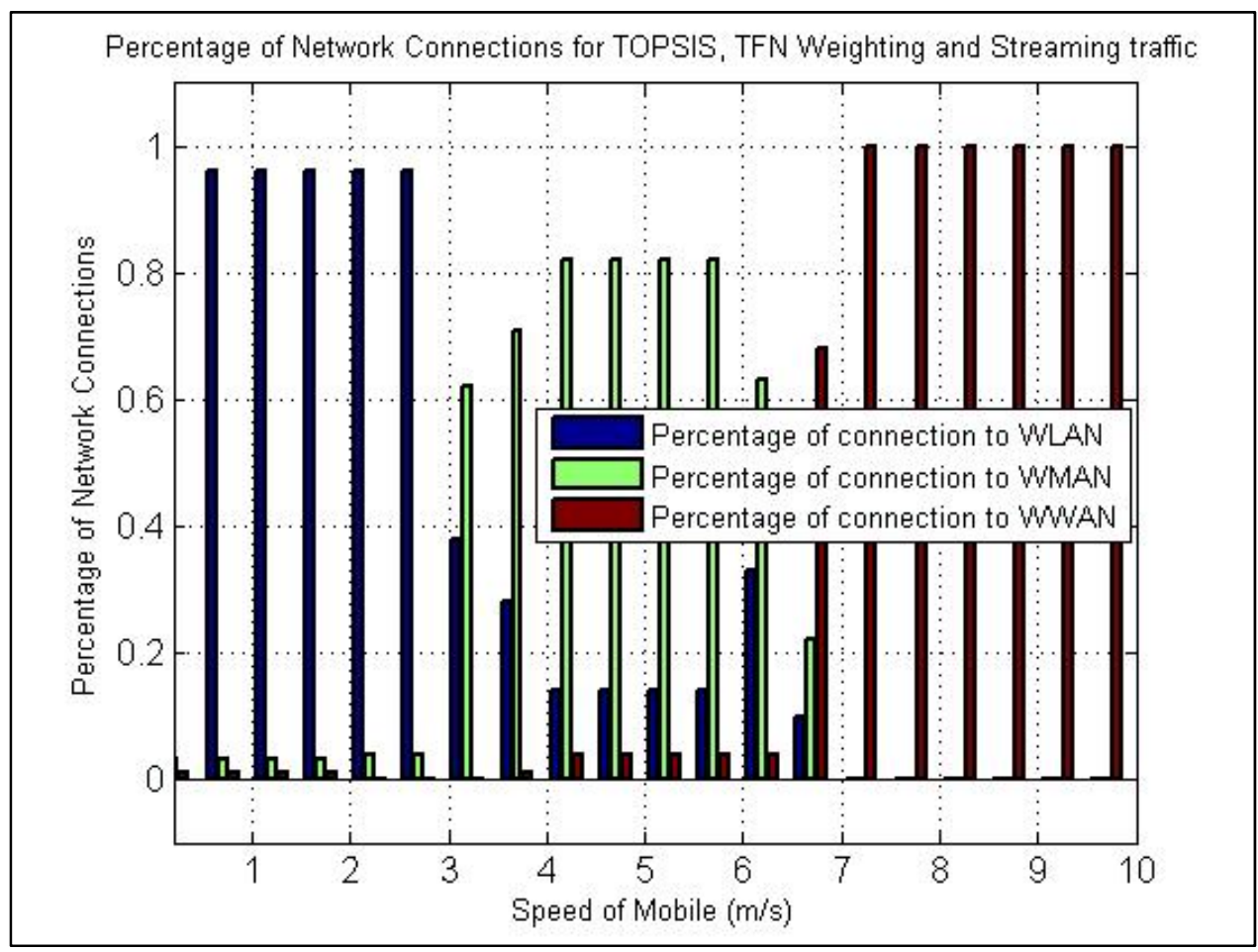

Figure 4.34: Percentage of NW-Connection, TOPSIS, TFN, for Streaming 
It can be observed from these figures that with minor differences, the percentages of connectivity towards a preferred wireless network for different traffic classes are almost the same. A $100 \%$ preference towards WWAN for the MS with higher speeds contrasting a $98 \%$ connectivity preference for WLAN can be observed. For slower speed, the percentage of connectivity to a preferred network provided by FAHP and TFN based schemes is exactly the same as AHP scheme, which is based on crisp data.

A comparison of Figure 4.23 with Figure 4.28 and Figure 4.32 shows that for Conversational traffic class, TOPSIS with AHP prefers WLAN at a mobile speed between $6-8 \mathrm{~m} / \mathrm{s}$. On the other hand, the fuzzy based weighting mechanism prefers WMAN and WWAN at these speeds, which should be the case. For Background traffic class, at higher speeds of the MS, the fuzzy based FAHP and TFN weight mechanisms give $100 \%$ preference towards WWAN (Figure 4.27 and Figure 4.31), whereas the crisp based AHP weighting scheme shows higher preference towards WLAN at higher speeds (Figure 4.25). Similar observations can be made for Interactive and Streaming traffic classes.

Based on the above discussion, it can be concluded that the performance of TOPSIS ranking algorithm utilizing fuzzy based weighting schemes (FAHP and TFN) is better, as compared with that of the crisp-value based AHP weighting mechanism.

\subsubsection{FTOPSIS BASED NETWORK SELECTION}

In a Fuzzy TOPSIS based network selection, the network attributes as well as their associated weights are treated as fuzzy values. The FTOPSIS is partially similar to 
TOPSIS with FAHP and to TOPSIS with TFN in terms of the weights of the network attributes that are based on fuzzy data.

Figures 4.35-4.38 depict percentages of connections towards a preferred network selected by FTOPSIS scheme. The percentage of network selection for the Background traffic class is shown in Figure 4.35. It can be seen from this figure that FTOPSIS shows higher preference towards WLAN for the MS moving with slow and medium speeds. Approximately $98 \%$ and $62 \%$ connectivity preferences for WLAN can be seen for slow and medium speed MS. WMAN with $37 \%$ connectivity preference can be observed as the second choice for a medium speed MS whereas $100 \%$ connectivity preference is shown for WWAN at higher speeds.

For Conversational and Interactive traffic classes, FTOPSIS prefers WWAN for medium and high speeds MS, with an average network connectivity of $60 \%$ and $100 \%$, respectively. Both of these traffic classes require a lower value of network delay and WWAN is fulfilling this requirement. On the other hand, WLAN is the preferred network for a slower moving MS. This can be seen from Figure 4.36 and Figure 4.37.

The behavior of FTOPSIS for Streaming traffic class is the same as Conversational and Interactive class with the exception of the MS moving with a medium speed. It can be seen from Figure 4.38, that the percentage of network connections for WMAN for a medium speed MS is around $60 \%$. As discussed earlier, the Streaming traffic class requires a higher throughput from the network. As can be seen from this figure, WLAN and WMAN fulfill this requirement for lower and medium MS-speed, respectively. 


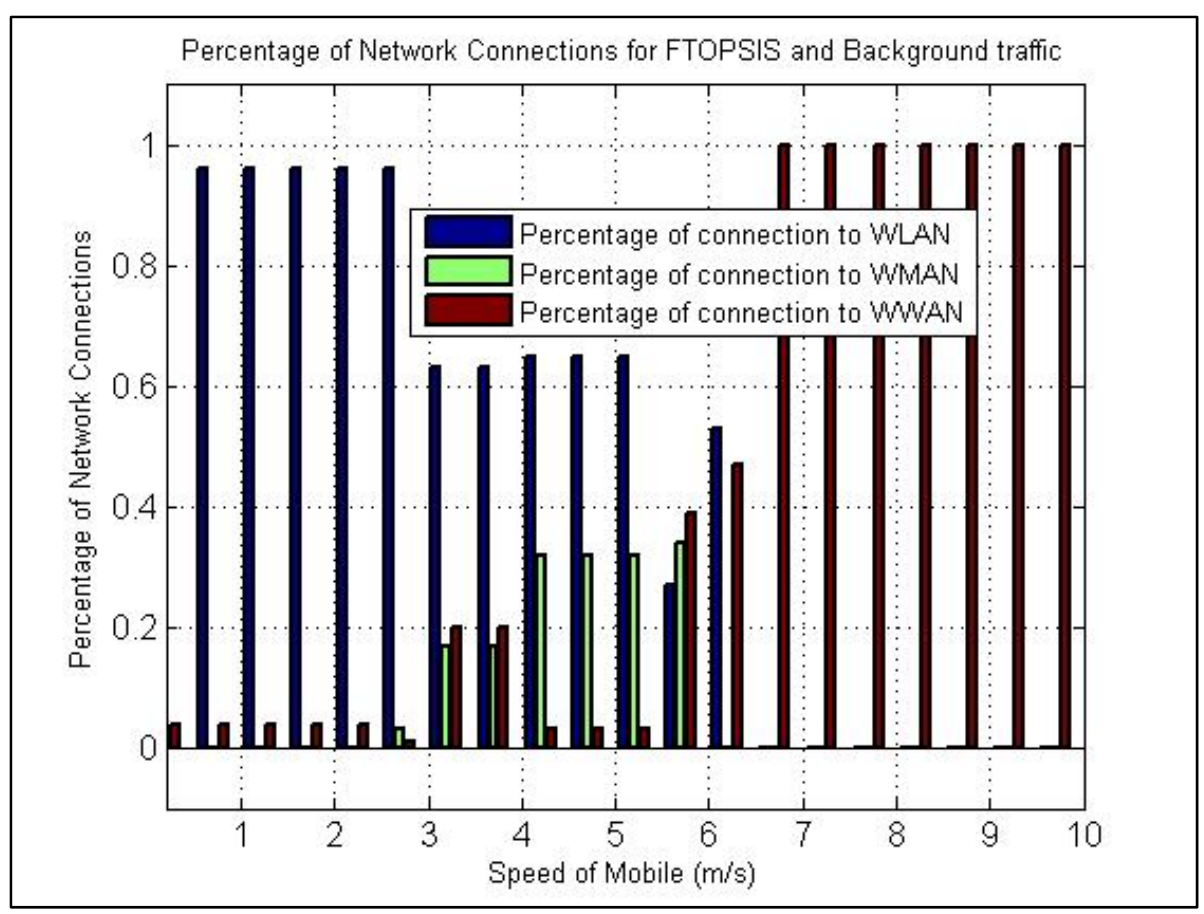

Figure 4.35: Percentage of NW-Connection, FTOPSIS for Background Traffic

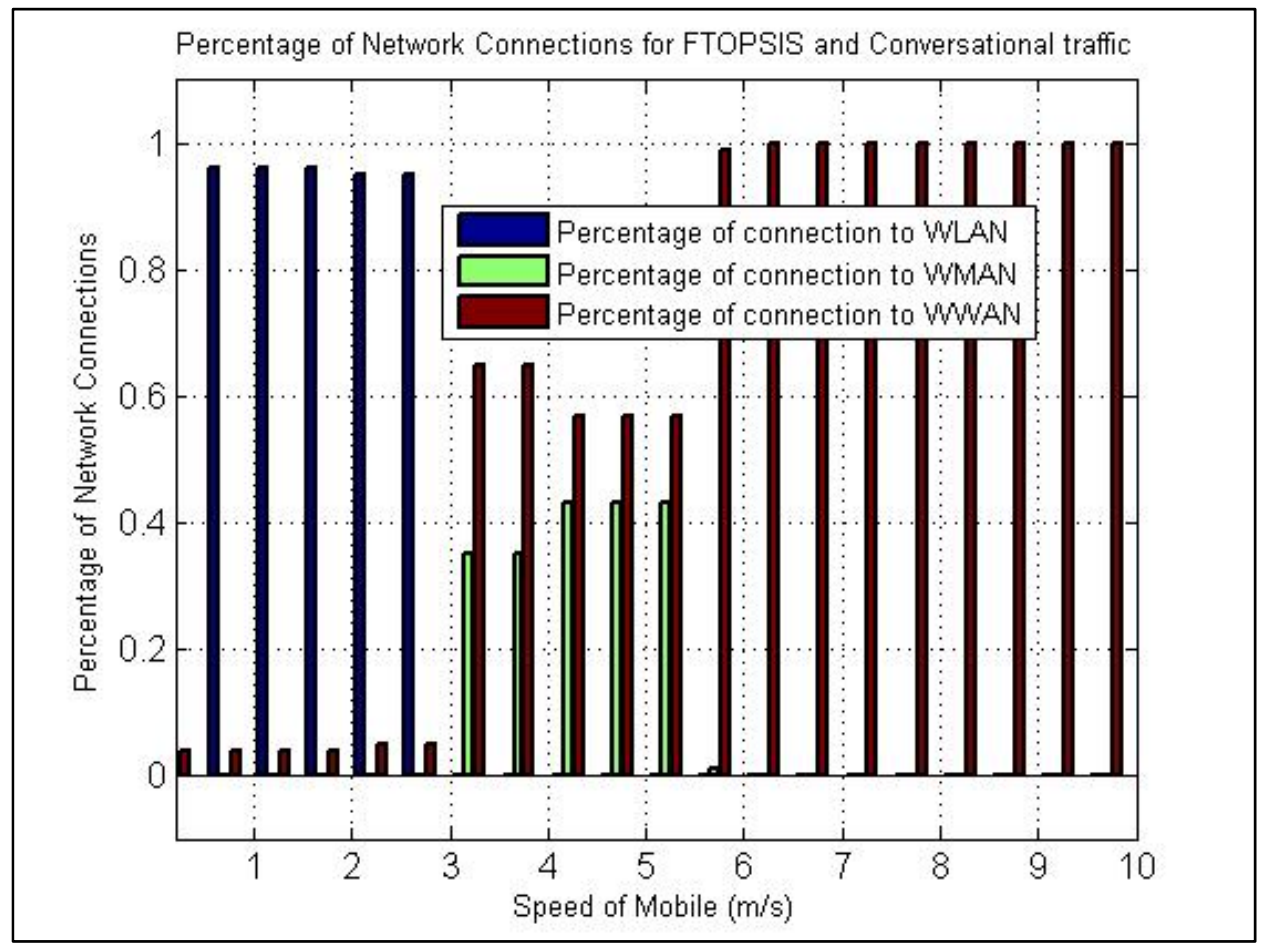

Figure 4.36: Percentage of NW-Connection, FTOPSIS for Conversational traffic 


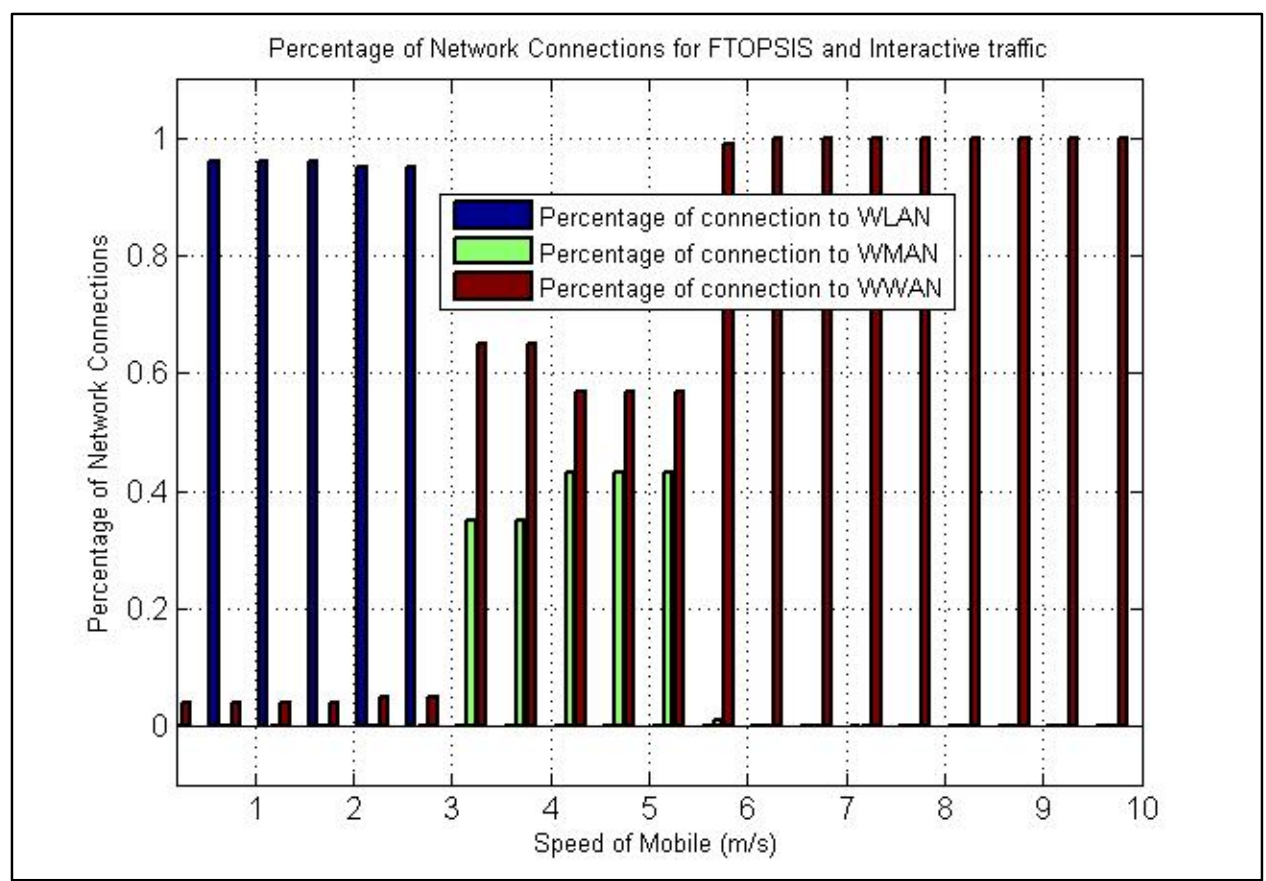

Figure 4.37: Percentage of NW-Connection, FTOPSIS for Interactive Traffic

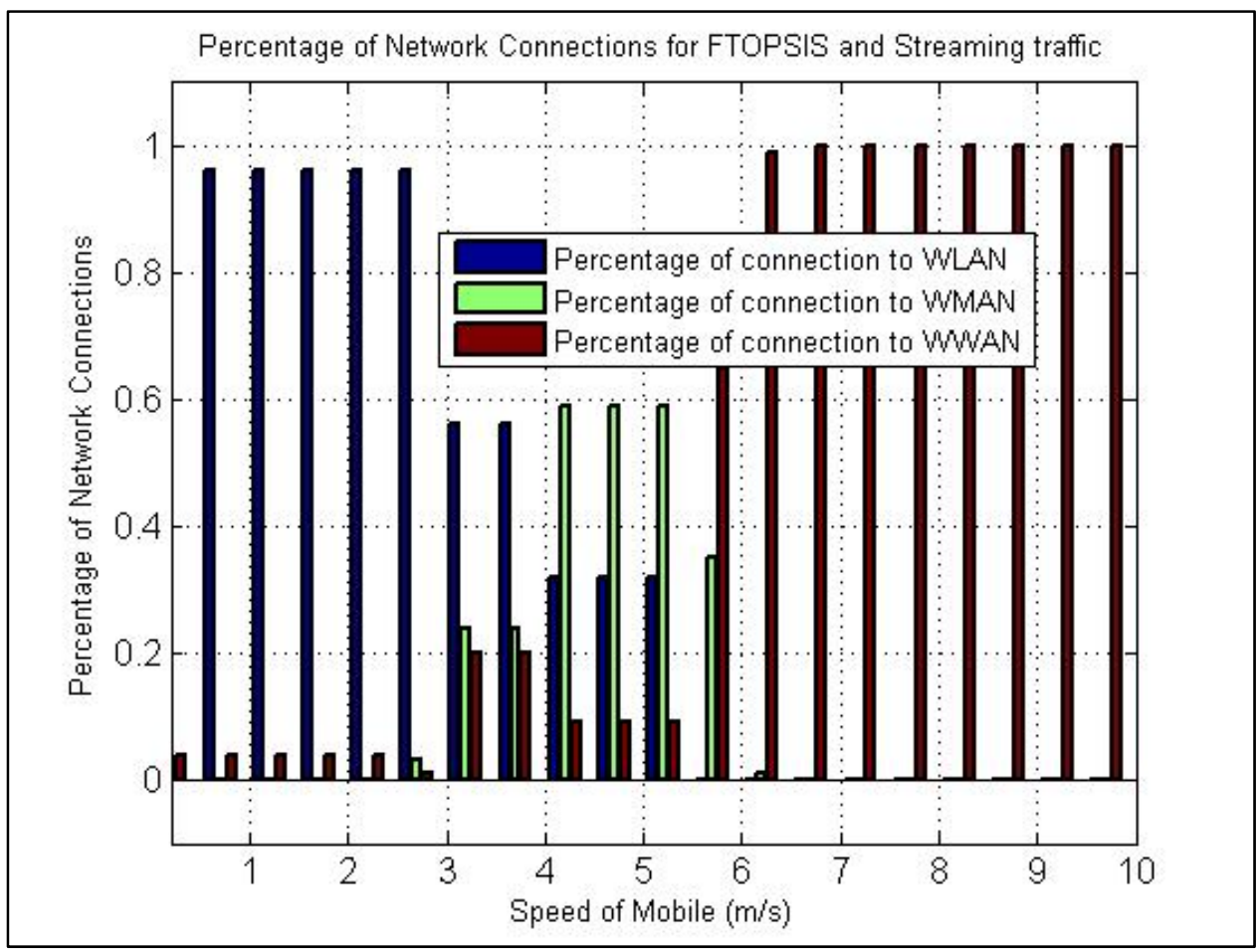

Figure 4.38: Percentage of NW-Connection, FTOPSIS for Streaming Traffic 


\subsubsection{FVIKOR BASED NETWORK SELECTION}

Similar to FTOPSIS, FVIKOR also utilize fuzzy data for both network attributes and their associated weights. Unlike the other ranking techniques discussed earlier, FVIKOR demonstrates a distribution of percentages for connectivity preferences among the available networks for different traffic types. This is depicted in Figures 4.39-4.42.

For Background traffic class, approximately $94 \%$ and $60 \%$ connectivity preferences towards WLAN can be observed for slower and high speed mobile, respectively. At medium speed, a strong competition between WLAN and WMAN can be observed from Figure 4.39, where the first preference is given to WMAN with approximately $42 \%$ of network connectivity. On the higher speed side, WWAN trails WLAN with a network connectivity of $38 \%$. An important trend that can be observed from this figure is that as the speed of the MS increases, the percentage of network connections to WLAN decreases from $94 \%$ to $60 \%$. FVIKOR gives higher connectivity preference for Conversational traffic class to WLAN for an MS moving with any speed. This is shown in Figure 4.40.

For Interactive traffic class, a mixed behavior can be observed where WLAN and WWAN are given higher connectivity preferences as compared with WMAN. This is depicted in Figure 4.41. At higher speed, approximately $60 \%$ of network connections preferred WWAN. 


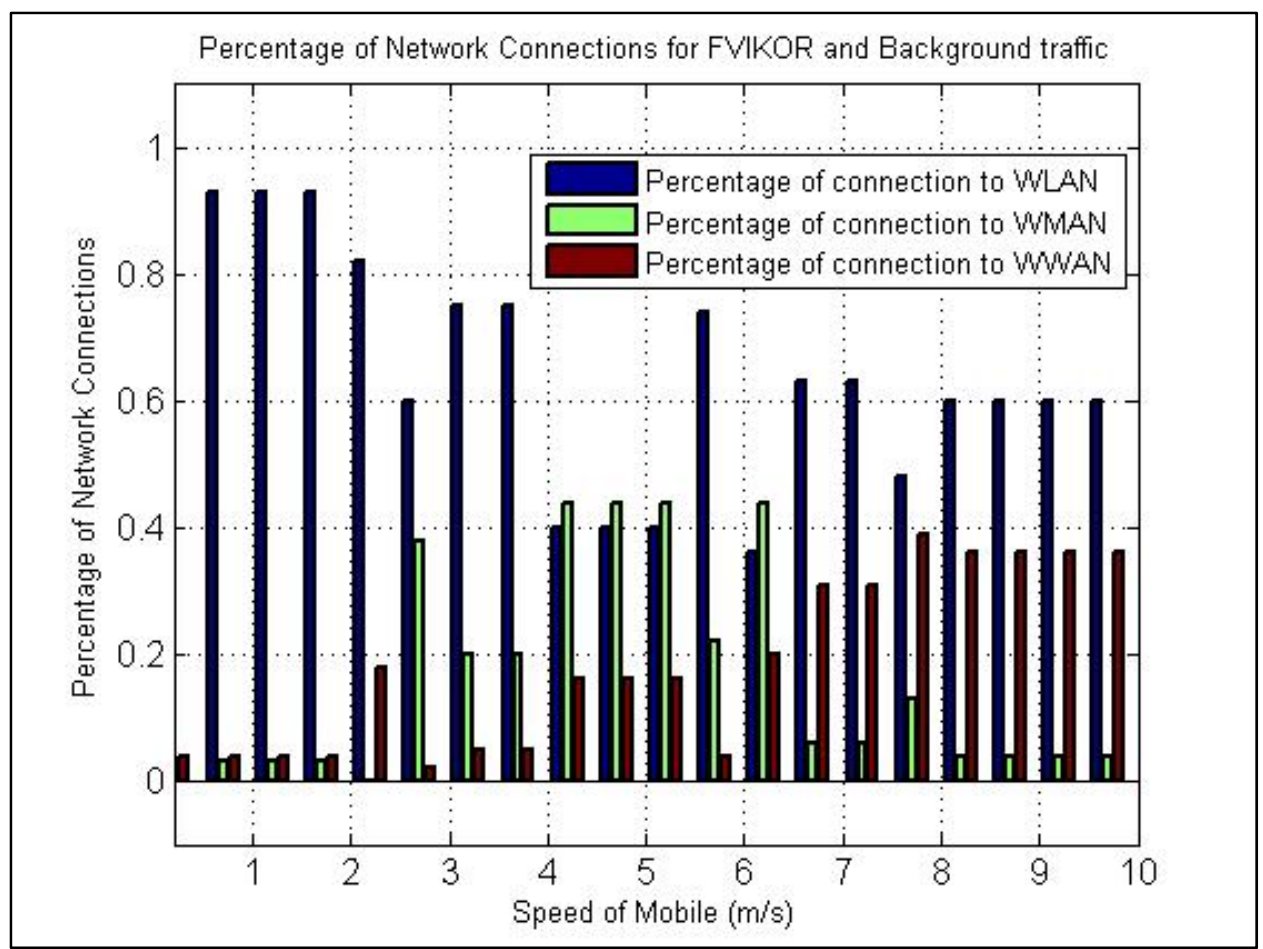

Figure 4.39: Percentage of NW-Connection, FVIKOR for Background Traffic

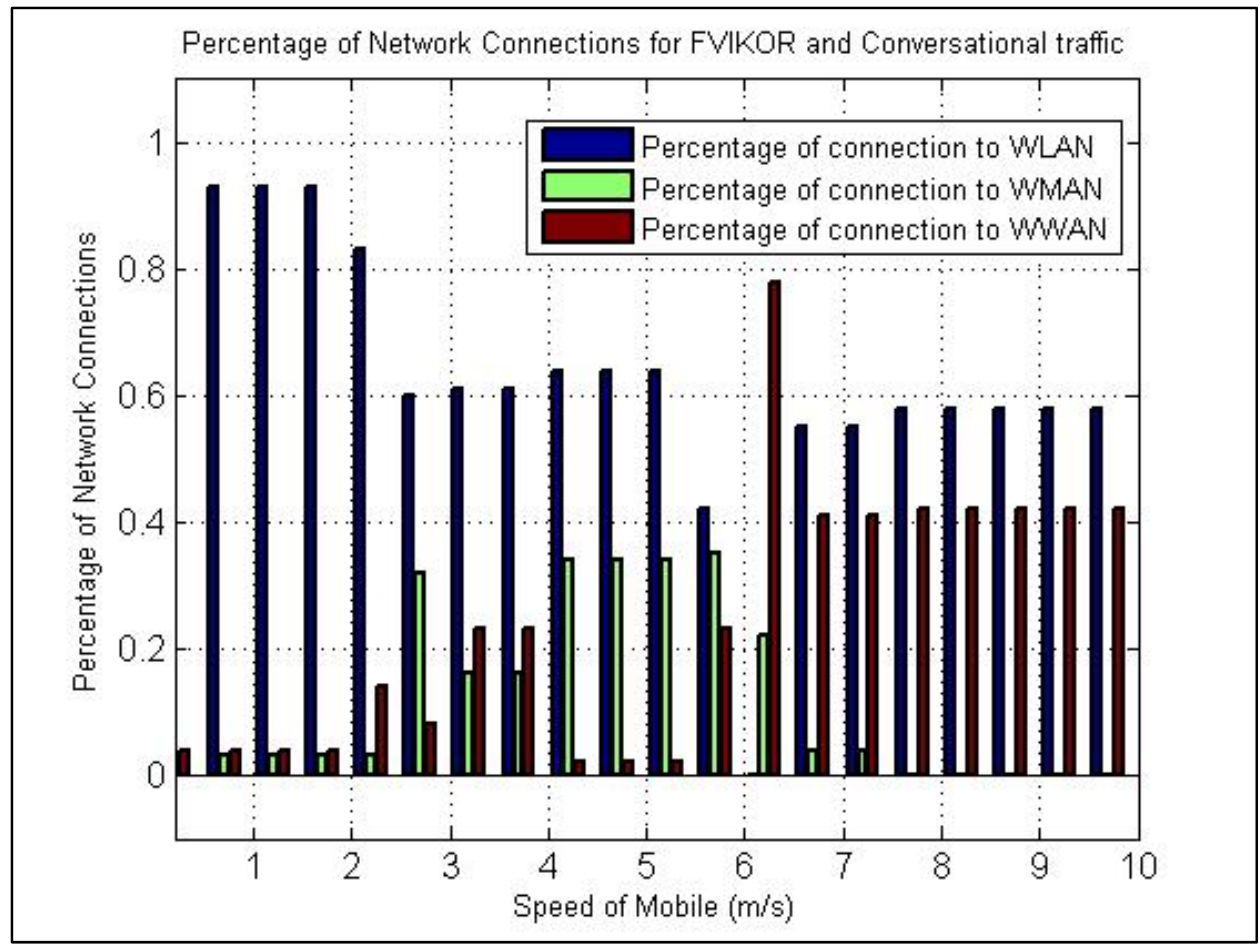

Figure 4.40: Percentage of NW-Connection, FVIKOR for Conversational Traffic 
Like Conversational traffic class, FVIKOR gives similar preferences to the Streaming traffic class. Streaming traffic class requires a higher value of throughput and WLAN is the network currently providing this higher value. As can be seen from Figure 4.42, WLAN is the preferred wireless network for any MS-speed whereas WMAN and WWAN are given second preferences by FVIKOR for an MS moving with medium to higher speeds, respectively.

\subsection{SIMULATION RESULTS: SINGLE MOBILE USER PREFERENCES}

In this section, different simulation results are presented based on the end-user's preferences in terms of cost and security. Once again, we still assume the presence of only one MS moving in a straight line with access to different wireless access networks. Simulation results for only Streaming traffic class is presented here, the results for the other traffic classes can be obtained in a similar fashion.

\subsubsection{TOPSIS BASED NETWORK RANKING WITH USER PREFERRED COST}

Figures 4.43-4.45 show a high end-user preference towards connecting to a less costly network. Based on the network parameters listed in Table 4.11, WLAN is the network that can fulfill this user requirement as it provides connectivity at a relatively low cost. It can be observed that in this case, the weighting schemes in general become irrelevant as the user assigns more weights towards the cost attribute. For the same reason, approximately $90 \%$ of the connections are towards WLAN, regardless of the MSspeed. On the other hand, since the overall QoS provided by WLAN is relatively weaker as compared with WWAN, approximately $10 \%$ connectivity preferences can be seen towards WWAN. 


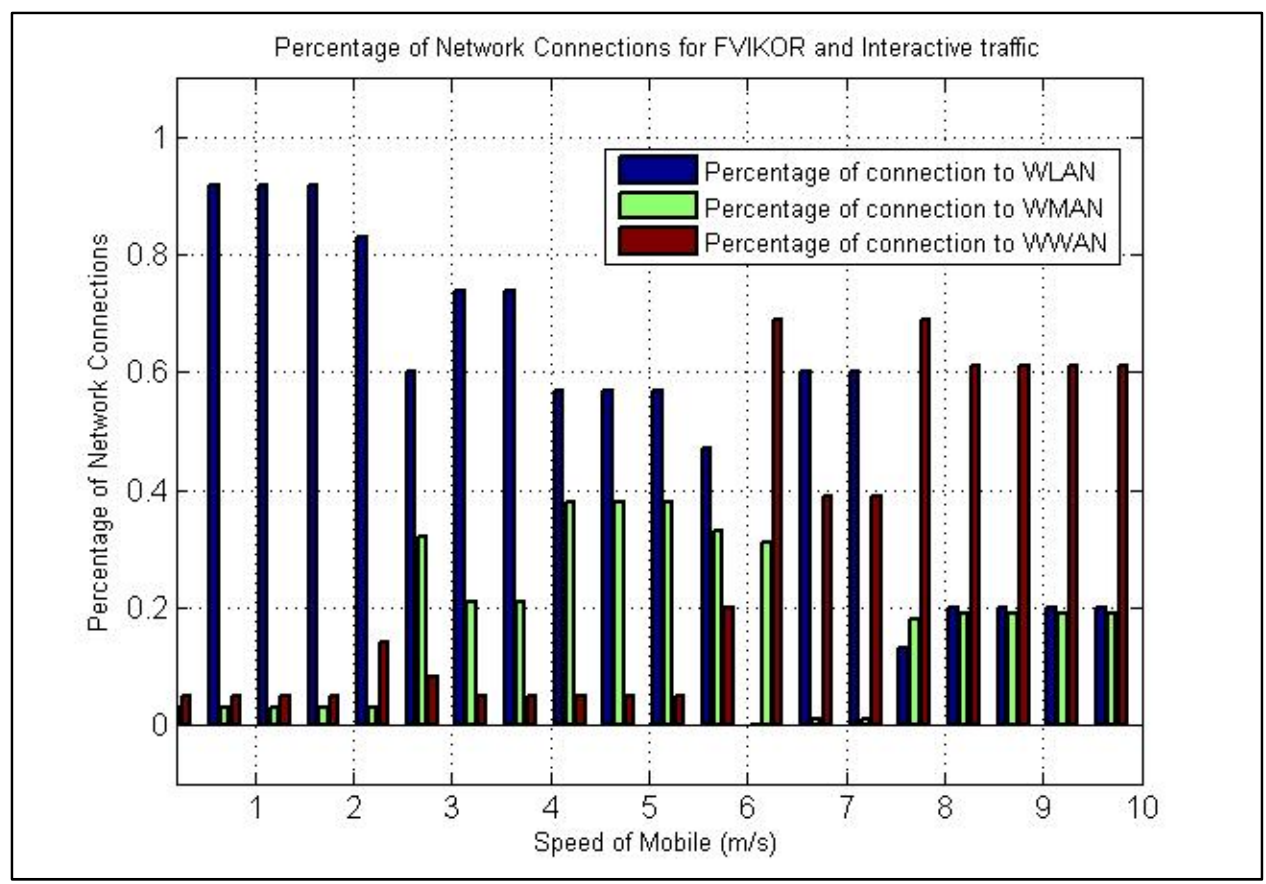

Figure 4.41: Percentage of NW-Connection, FVIKOR for Interactive Traffic

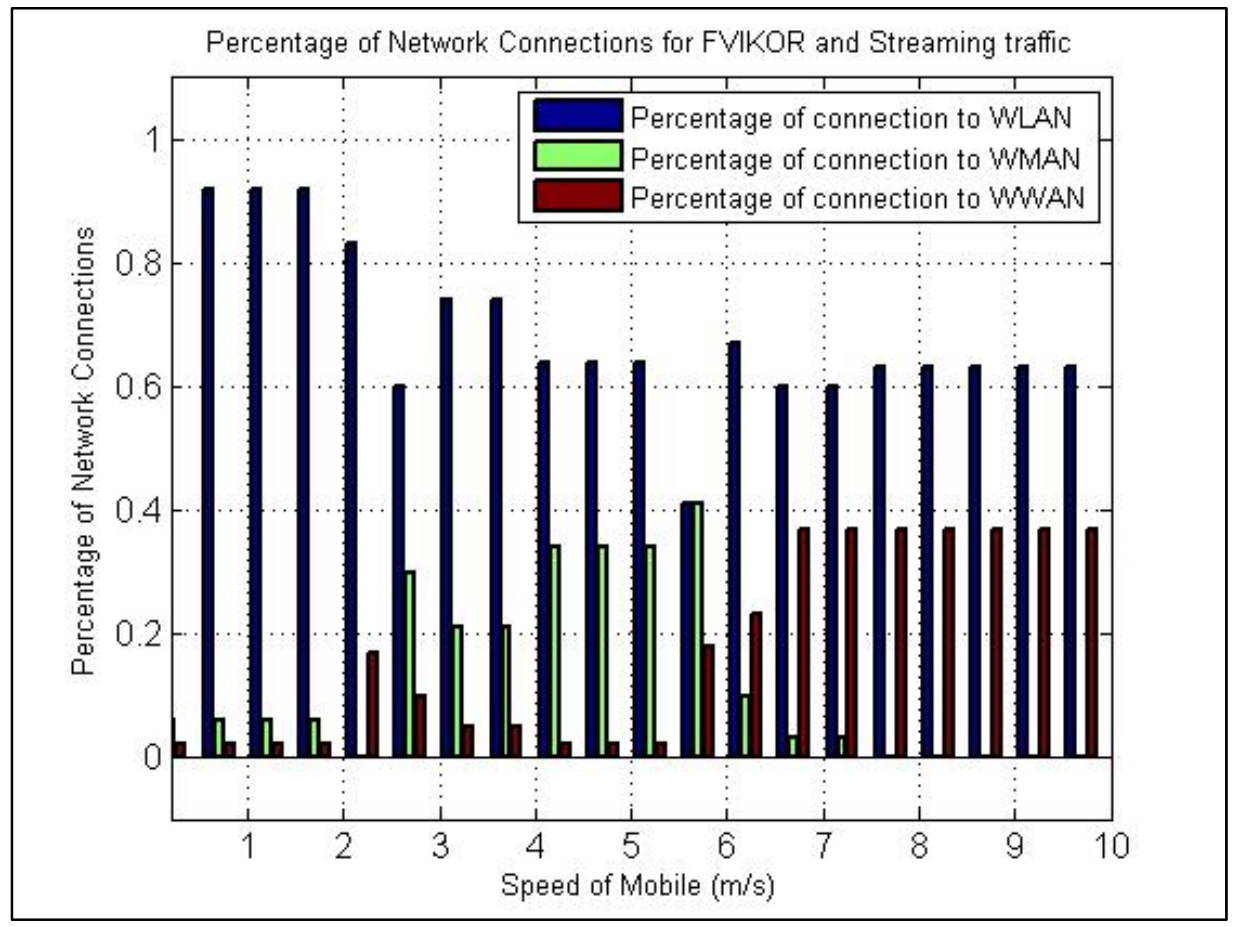

Figure 4.42: Percentage of NW-Connection, FVIKOR for Streaming Traffic 


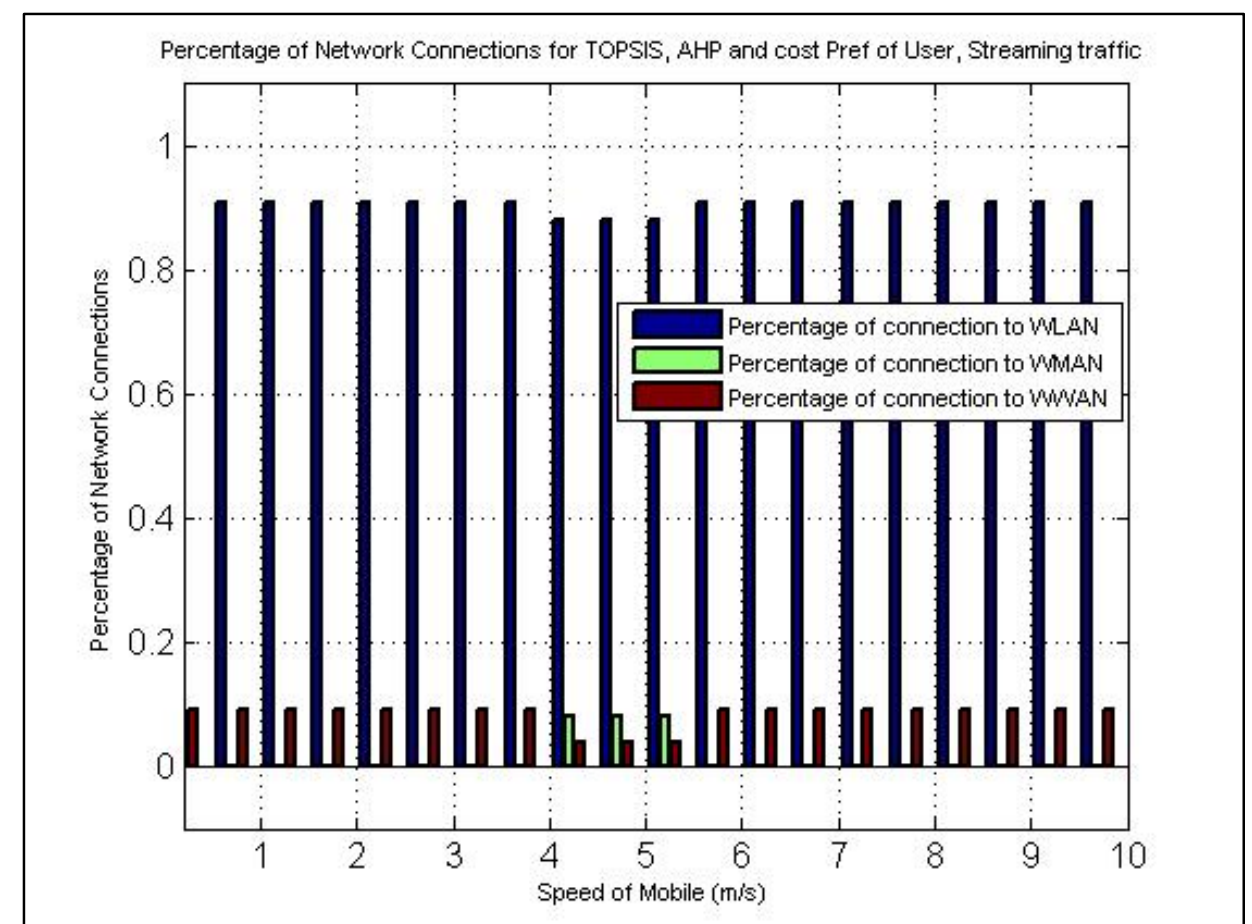

Figure 4.43: Percentage Connections, Preferred Cost, TOPSIS, AHP, Streaming

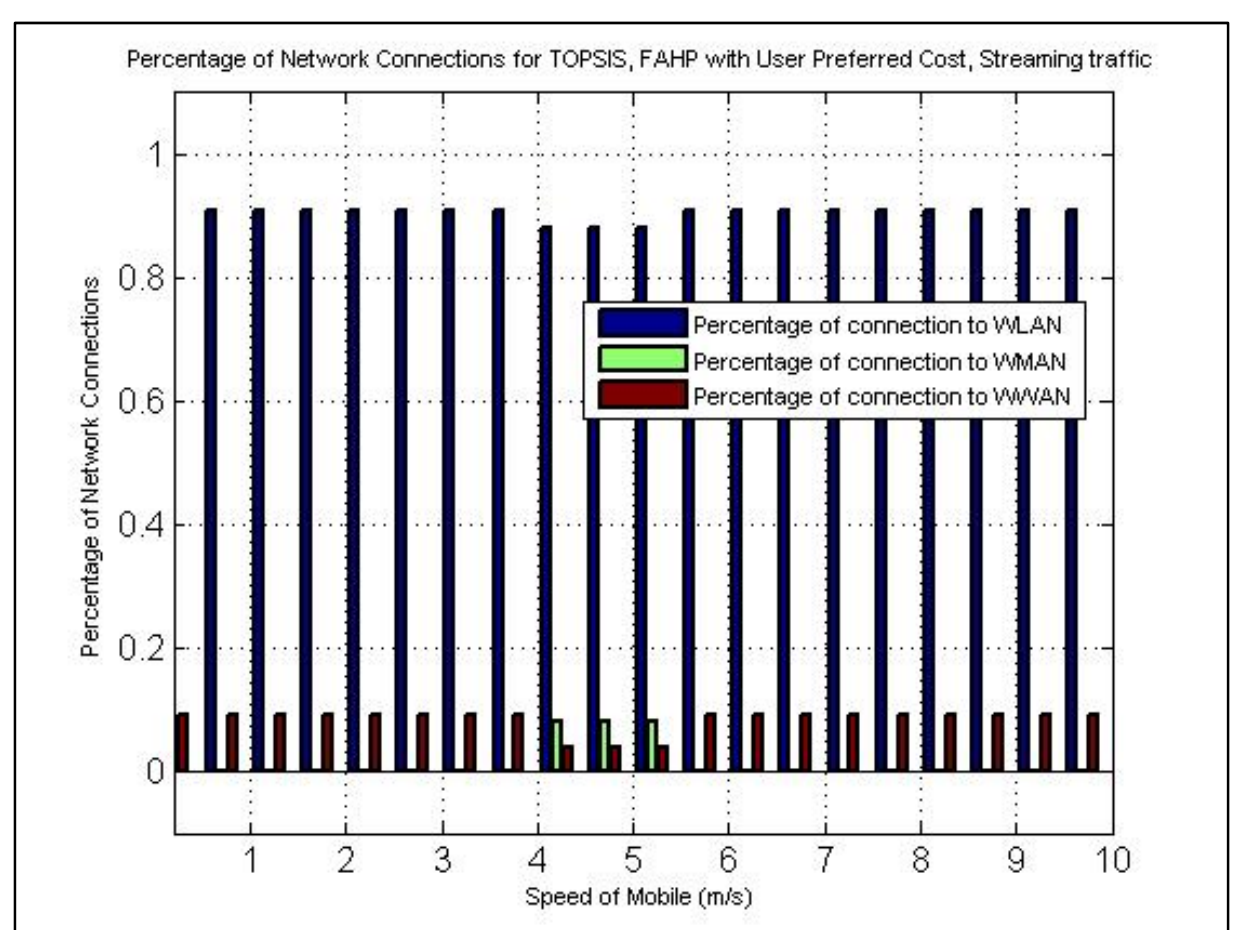

Figure 4.44: Percentage Connections, Preferred Cost, TOPSIS, FAHP, Streaming 


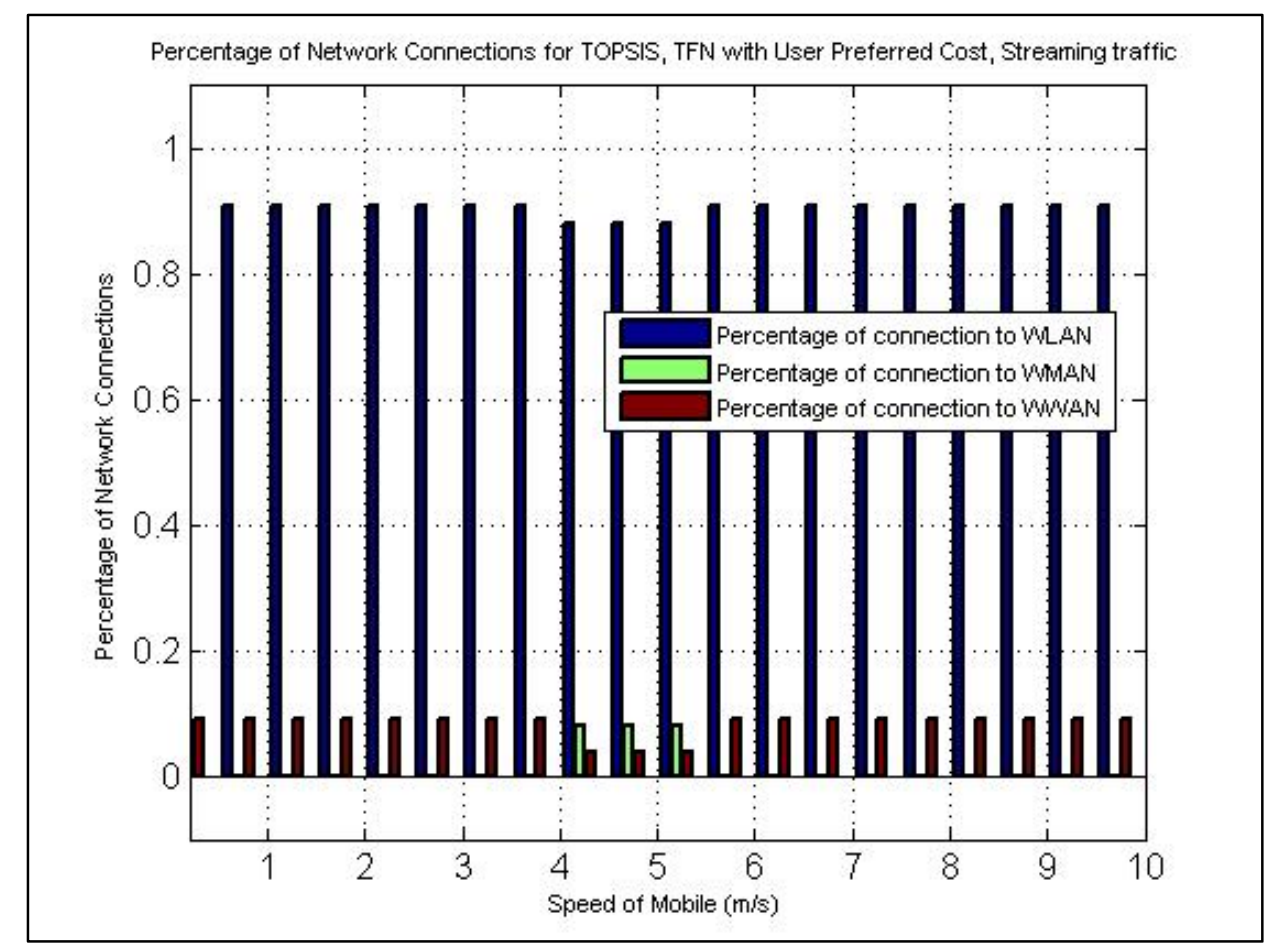

Figure 4.45: Percentage Connection, Preferred Cost, TOPSIS, TFN, Streaming

\subsubsection{TOPSIS NETWORK RANKING WITH USER PREFERRED SECURITY}

In the scenario where the end-user prefers a network with a higher offering of security, $100 \%$ connectivity preference for WWAN can be seen from Figures 4.46-4.48. Once again, the weighting scheme does not play an important role here since the user assigns higher weights towards security attribute. These results can be verified by observing the chosen parameters in Table 4.11 where a higher security offering with relatively better QoS parameters is offered by WWAN. Since the WWAN can fulfill the requirements for all traffic classes while providing a higher security, it is the preferred network for the MS travelling at any speed. 


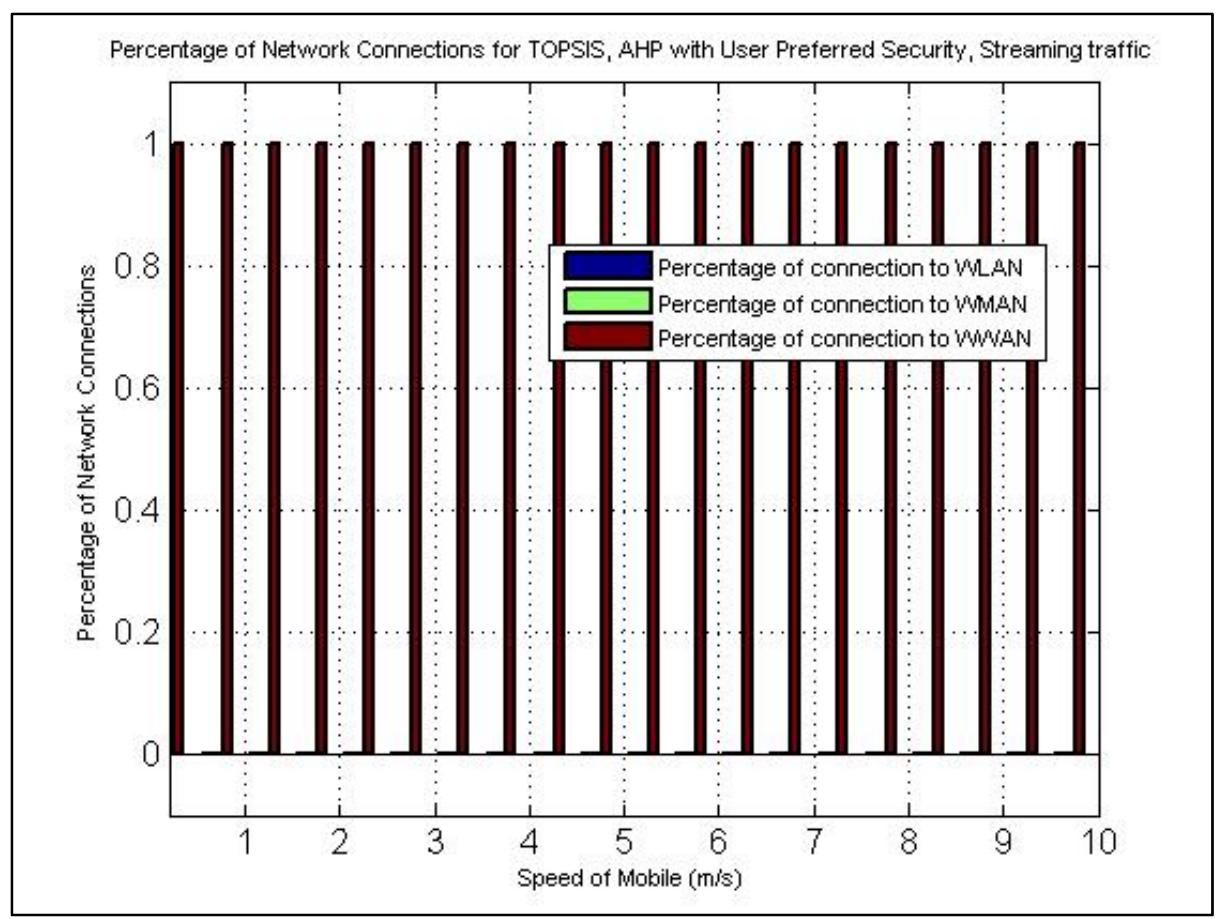

Figure 4.46: Percentage of NW-Connection for Preferred Security, TOPSIS, AHP, Streaming

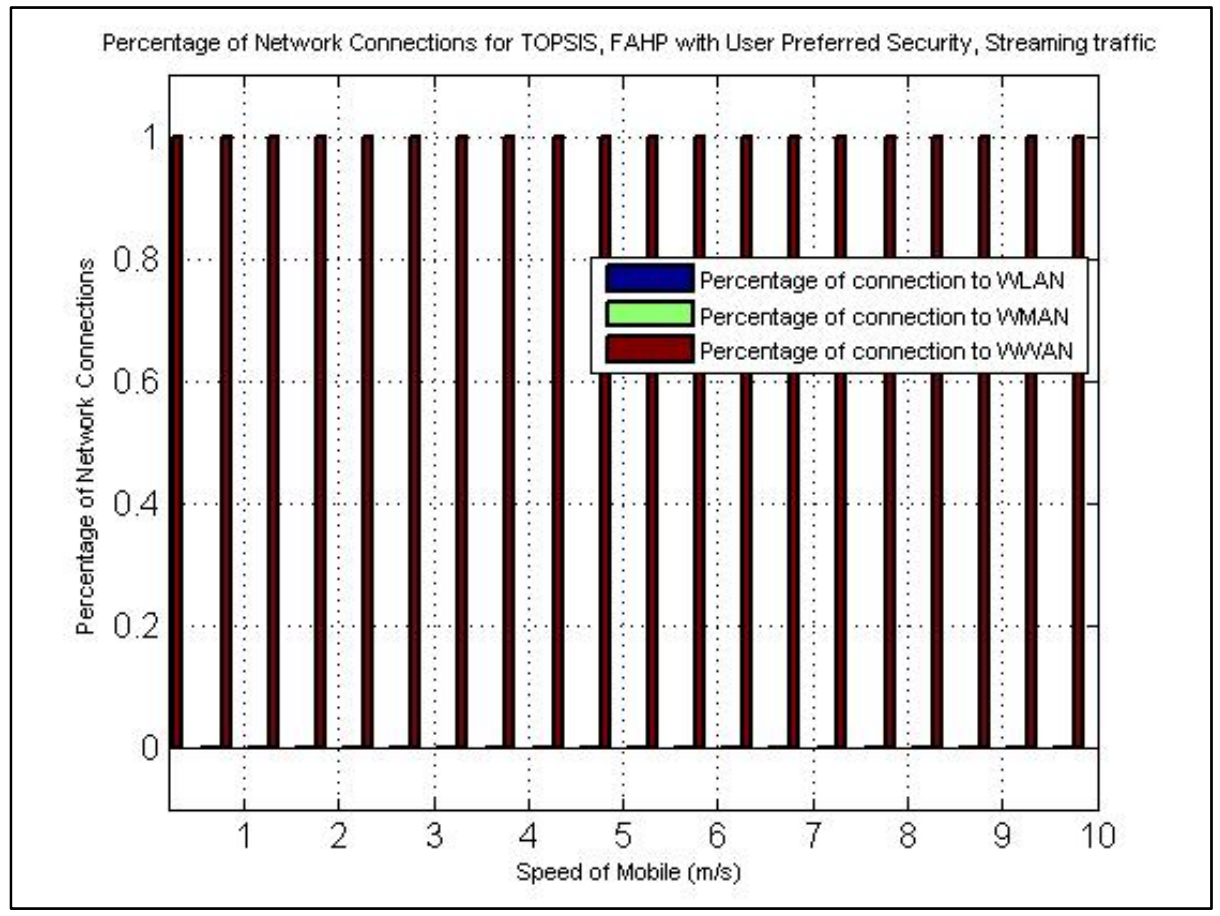

Figure 4.47: Percentage of NW-Connection for Preferred Security, TOPSIS, FAHP, Streaming 


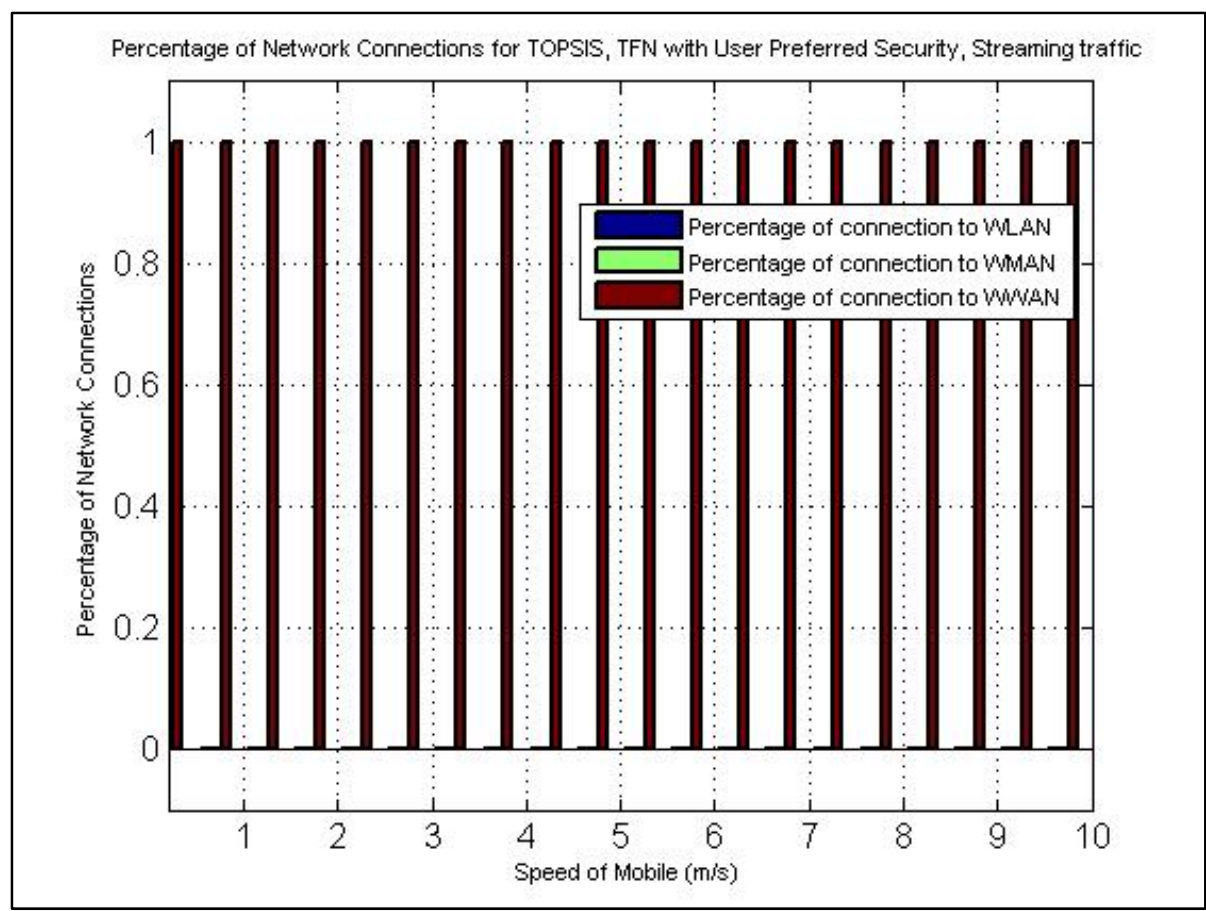

Figure 4.48: Percentage of NW-Connection for Preferred Security, TOPSIS, TFN, Streaming

\subsubsection{FTOPSIS AND FVIKOR BASED NETWORK RANKING WITH USER PREFERRED COST}

Figures 4.49-4.50 show percentages of network connection that are assigned to the MS with preferred cost for FTOPSIS, and FVIKOR schemes, respectively. These results are different as compared with the ones where TOPSIS based schemes are used (Figures 4.43-4.45). FTOPSIS based scheme respects the users' preference of cost by assigning the MS with a slow to medium speed to WLAN. For higher MS-speed, 100\% of the network connection to WWAN can be seen. This is because for a higher speed MS, VHITS prefers WWAN. Even though the user wants low cost network and WLAN can fulfill this requirement for the most part, FTOPSIS intelligently assigns a higher speed MS to WWAN to guarantee continuity and quality of the currently utilized service. 


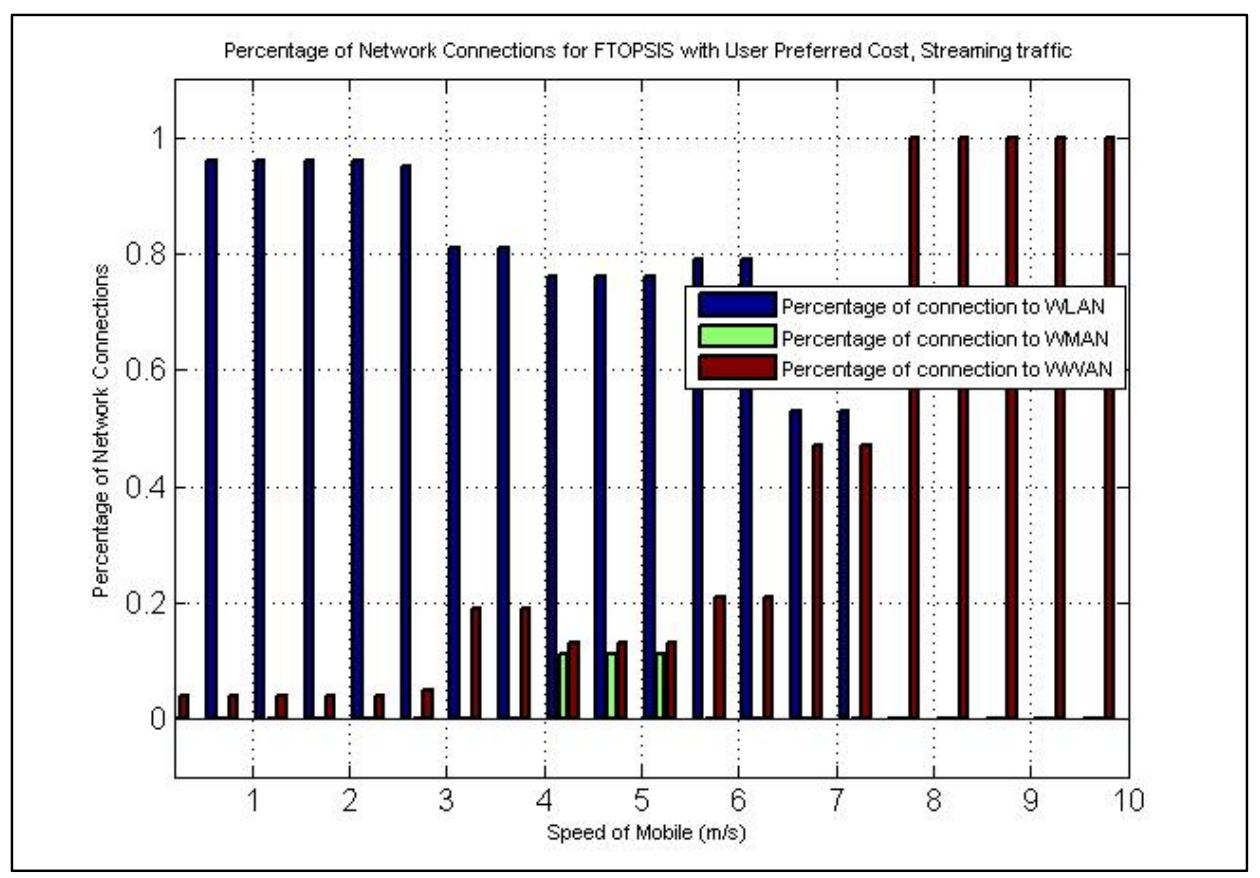

Figure 4.49: Percentage of NW-Connection for Preferred Cost, FTOPSIS, Streaming

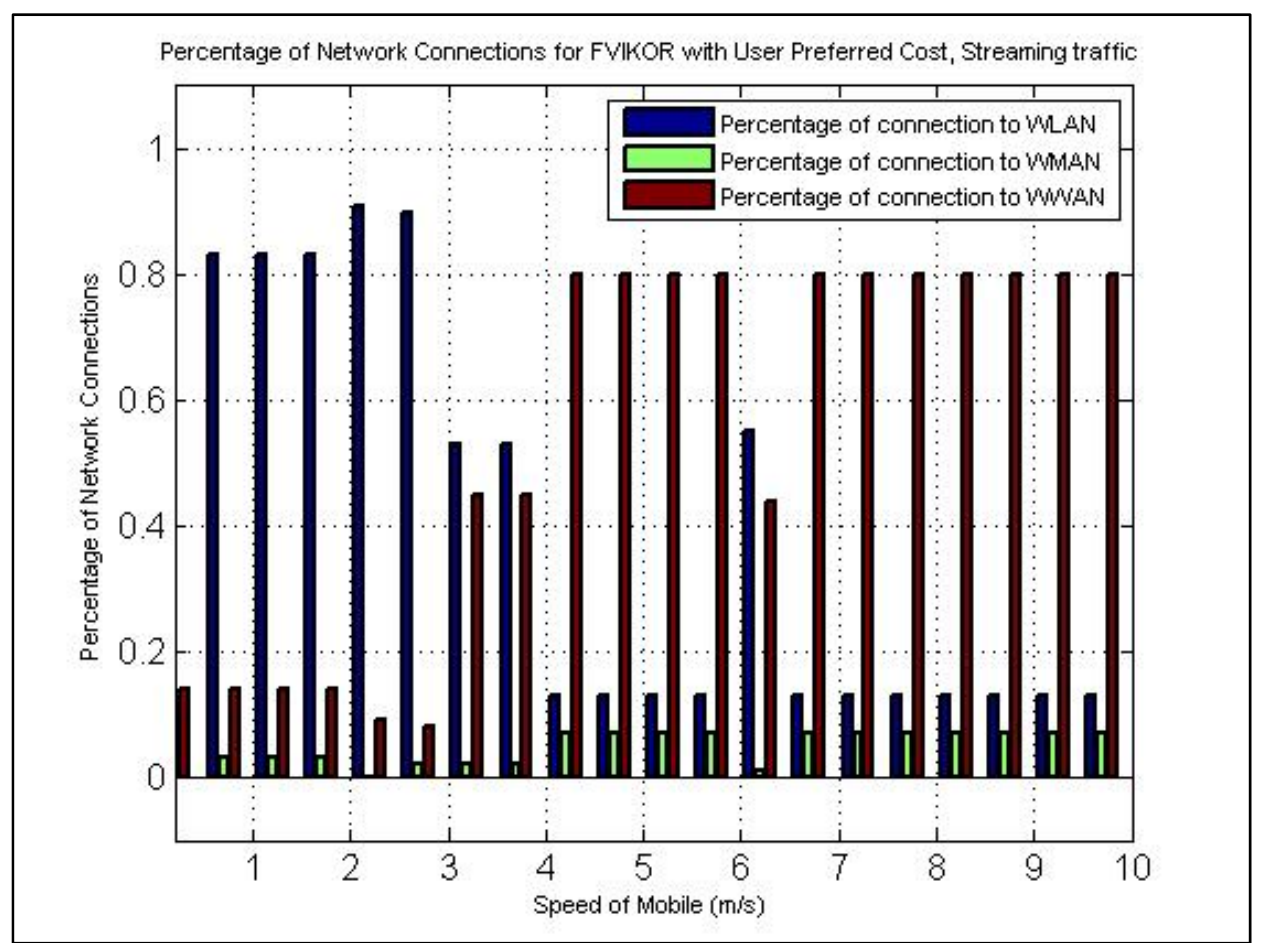

Figure 4.50: Percentage of NW-Connection for Preferred Cost, FVIKOR, Streaming 


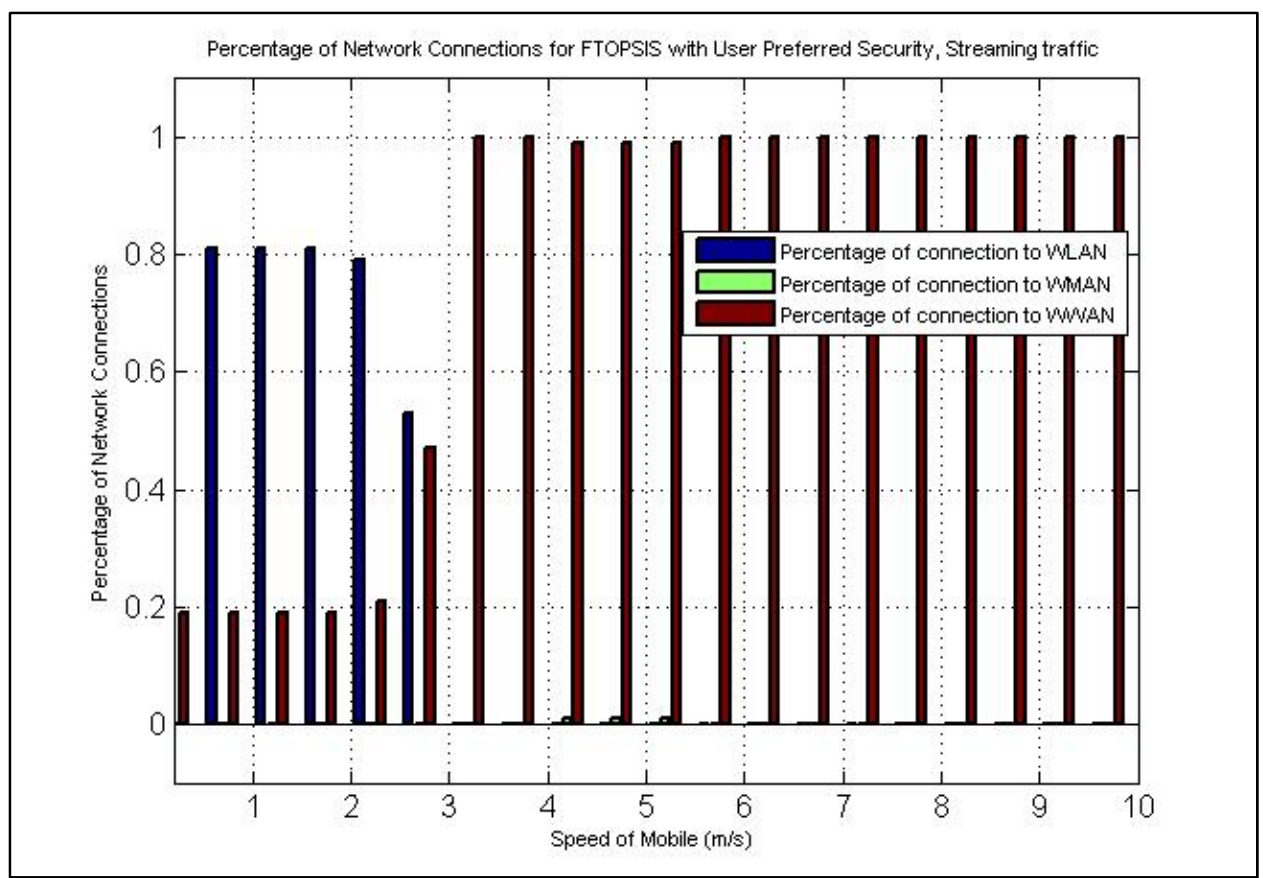

Figure 4.51: Percentage of NW-Connection for Preferred Security, FTOPSIS, Streaming

FVIKOR on the other hand, behaves the same but shows a different pattern for the MS moving with medium speed. As can be seen from Figure 4.50, WLAN is the preferred network for the MS moving at slow speed as it fulfills the low cost user preference, whereas WWAN is chosen for a medium to higher speed MS.

\subsubsection{FTOPSIS AND FVIKOR BASED NETWORK RANKING WITH USER PREFERRED SECURITY}

The percentages of network connection for FTOPSIS and FIKVOR with userpreferred security are depicted in Figures 4.51 and 4.52, respectively. As per the chosen network settings, WWAN provides higher security as compared with any other network. FTOPSIS chooses WWAN for a medium to higher speed MS. For slower speed, a connectivity preference of $20 \%$ can be seen towards WWAN. 


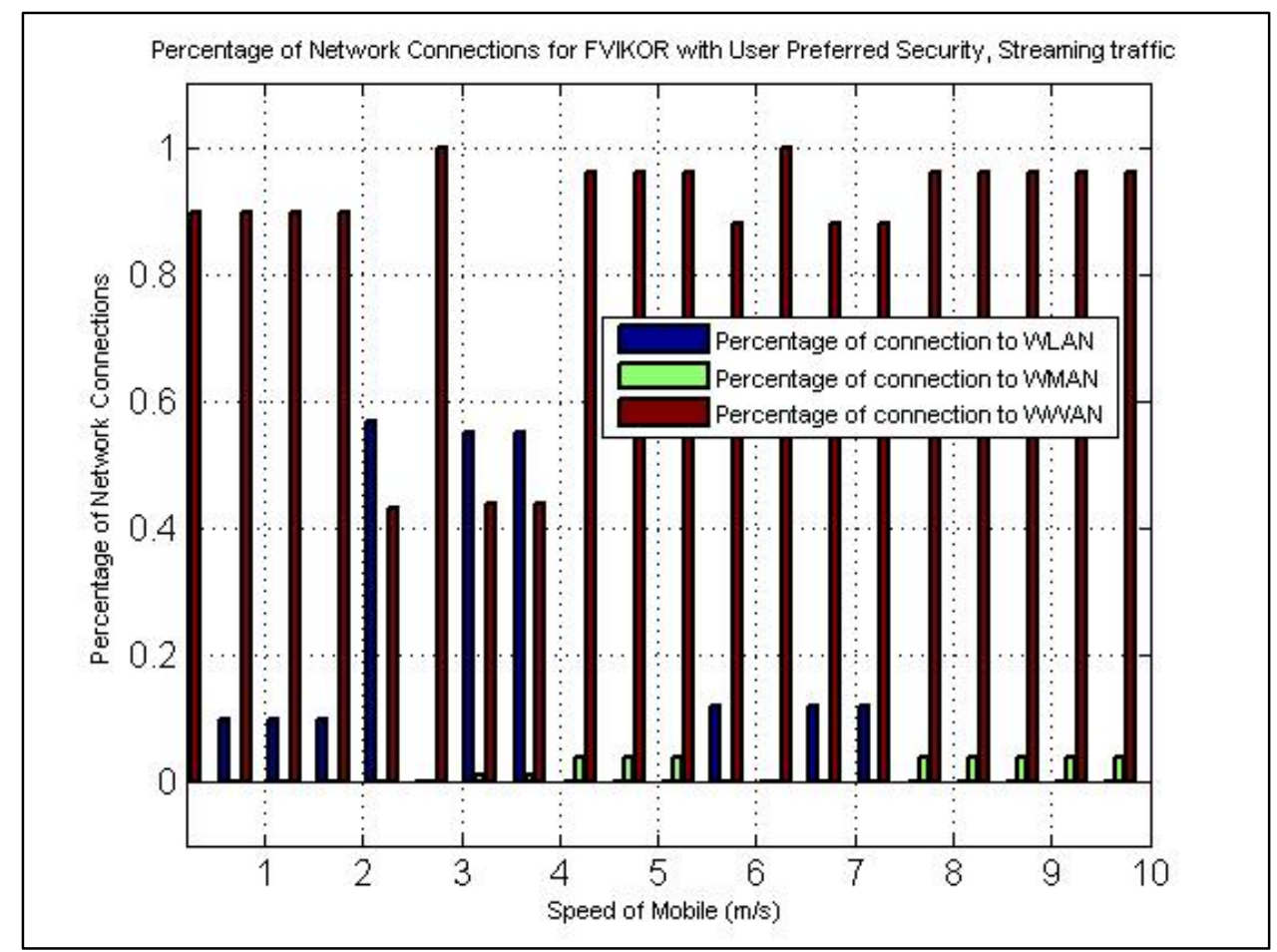

Figure 4.52: Percentage of NW-Connection for Preferred Security, FVIKOR, Streaming

A higher connectivity preference towards WWAN for the MS moving with any speed can be seen in Figure 4.52 where the selection is based on FVIKOR.

\subsection{SIMULATION RESULTS: MULTIPLE MOBILE USERS}

This section presents the evaluation of the proposed scheme assuming the presence of multiple MSs distributed uniformly throughout the system. As explained earlier, Poisson distribution is used to control the call arrival and departure for all MSs to/from the simulation system. The direction of movement and the speed of MSs are randomly chosen. This means that at a given moment in time, there are multiple MSs in the simulation system that are within the coverage area of multiple wireless access technologies and are either moving towards or away from BSs/APs. Note that the multi- 
user environment is uncontrolled as compared to single-user where the assumption is a single-user moving in a straight line crossing several cells with different coverage areas.

The following metrics are considered to evaluate the proposed scheme.

- Average Outage Probability: The average outage probability is a statistical measure that defines the probability of an MS failing to receive a signal with adequate quality at a particular location. This outage occurs when the SINR of an MS falls below a prescribed threshold. The proposed scheme is evaluated using this metric. The necessity of handoff and the decision to select the best network as the target of handoff is related to this metric. A lower value of this metric indicates a high number of MSs that have received adequate signal strength, which in turn, translates into intelligent and efficient network selection decisions made by the proposed scheme.

- Average New Call Blocking Probability: The new call blocking probability is the likelihood that the new call/session that is initiated cannot be completed due to unreachable signal or unavailable channel within a cell. Mathematically, this can be defined as the ratio of new-callsconnect rejects and the total new-calls-connect requests. From the perspective of end-user satisfaction, this factor is not as critically important as the handoff call blocking probability that deals with active calls/sessions. This metric cannot be used to evaluate VHITS necessity estimation scheme since for any new service requests, the VHITS necessity estimation module is skipped and VHITS only executes the 
VHITS target network selection mechanism. Hence this metric is used to evaluate the VHITS Target network selection module.

- Average Handoff Blocking Probability: The handoff blocking probability, also called forced termination probability, is the probability that the requested handoff to the target network is blocked. This occurs due to many reasons including the sudden drop of signal and non-availability of a channel or other resources within a cell. Mathematically, this can be defined as the ratio between rejected handoffs and the total requested handoffs in a system. This metric is considered more important than the blocking probability of new calls because the call/session is already active, and the QoS is more sensitive for the handed-off calls. This importance is basically from the end-user perspective because loss of connections during active calls/sessions are worse than new call rejects, which in turn result in reduced overall satisfaction of the end-user. This metric is used to evaluate the overall VHITS scheme that involves handoff initiation and target selection.

- Average Handoff Rate: The handoff rate is defined as the number of handoffs that the MS has performed during a call connection. The metric is critically important as it affect the quality of the ongoing service. A lower value of this metric is desirable to maximize end-user satisfaction in terms of guaranteed continuity and quality of service. It is important to mention that the average handoff rate is directly proportional to the MS- 
battery consumption. The higher the handoff rate, the more quickly the MS-battery drains.

- Percentage of Network Connections based on Average Call Arrivals per Cell: This metric is used to compare the percentages of network connections that our scheme prefers for moving MSs utilizing different types of traffic classes. A higher value of this metric indicates intelligent and efficient network selection decisions by the proposed scheme.

The proposed scheme, using the above metrics, is evaluated using the traditional RSS based reference algorithm combined with the network's traffic loading conditions. Note that the RSS based algorithm is used mainly for benchmarking purposes and not necessarily for comparing the performance of the proposed scheme. These evaluations are done based on the average number of arrived calls in each cell with multiple MSs moving randomly at a speed of $1 \mathrm{~m} / \mathrm{s}, 5 \mathrm{~m} / \mathrm{s}$, and $9 \mathrm{~m} / \mathrm{s}$, respectively. As depicted in Table 4.12, the average number of calls per cell is random and varies from 1-10. Note that the simulations are shown only for Conversational traffic class and similar results can be obtained for other traffic classes as well.

\subsubsection{AVERAGE OUTAGE PROBABILITY WITH TOPSIS BASED SELECTION}

Figure 4.53 shows the average outage probability based on TOPSIS and crispbased AHP weighting scheme. It can be observed that MSs moving with any speed, the average outage probability of TOPSIS with AHP is better than the traditional RSS based scheme. For slower moving MSs, the TOPSIS with AHP has an outage probability below $20 \%$. For MSs moving with medium and high velocities, the outage probability increases 
as the number of average call arrival per cell increases. For higher speed MSs with the maximum number of average call arrival per cell, the outage probability for TOPSIS with AHP scheme is approximately $41 \%$ as compared to RSS based that is around $49 \%$.

The outage probabilities calculated using TOPSIS with fuzzy based FAHP and TFN weighting schemes are shown in Figures 4.51-4.55. A minor improvement over TOPSIS-AHP scheme can be observed for both slower and faster moving MSs. With maximum loading and at higher speeds, TOPSIS with FAHP, with an outage probability of less than $40 \%$, performs better than both TOPSIS with AHP and TOPSIS with TFN schemes.

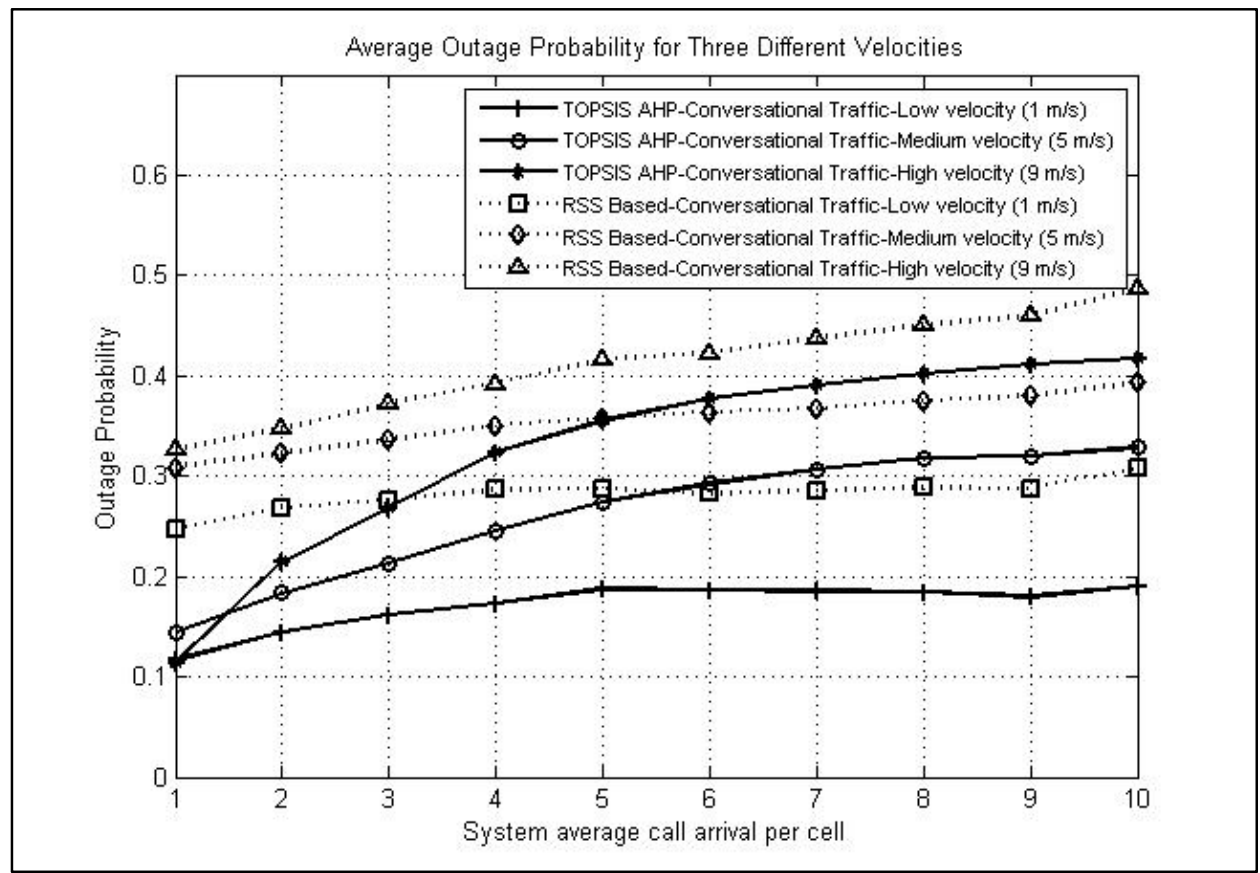

Figure 4.53: Average Outage Probability, TOPSIS, AHP, Conversational 


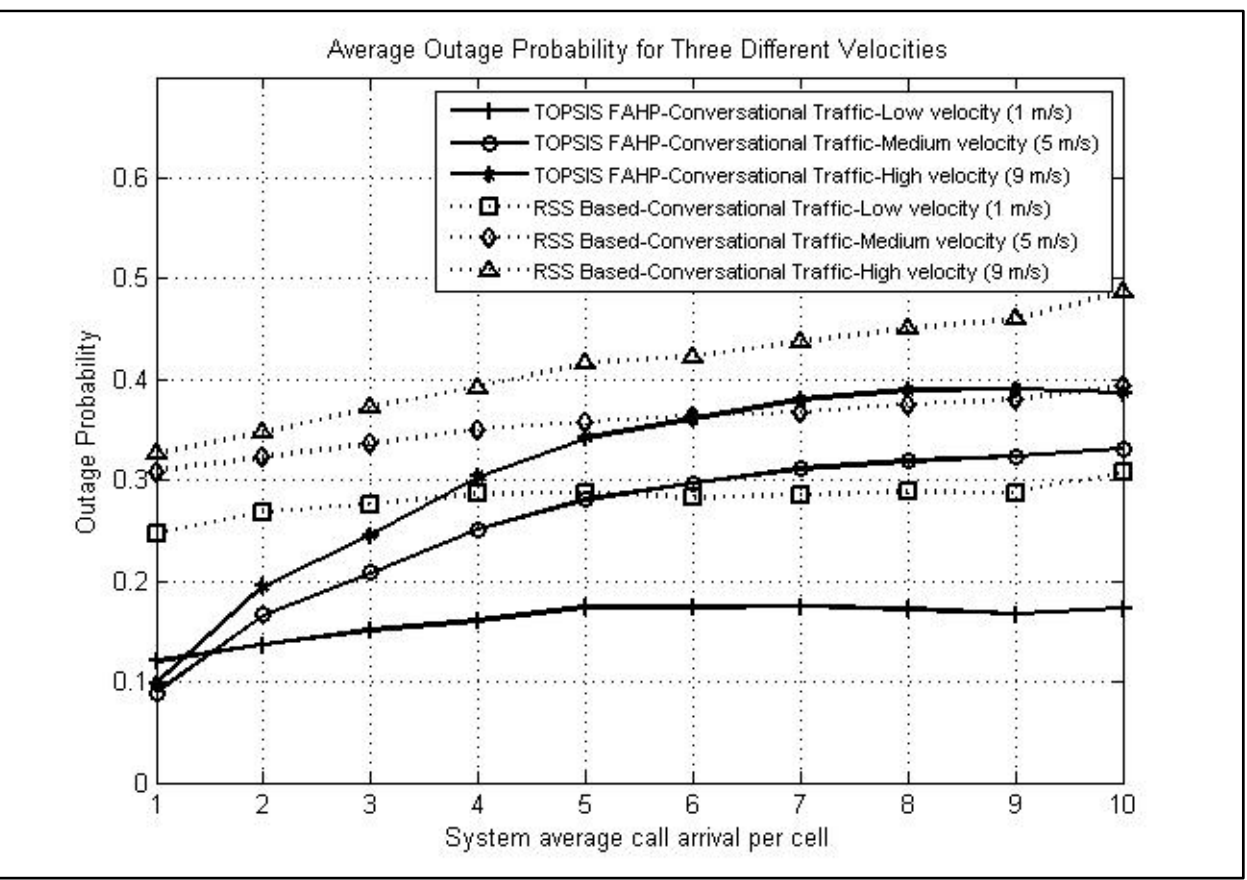

Figure 4.54: Average Outage Probability, TOPSIS, FAHP, Conversational

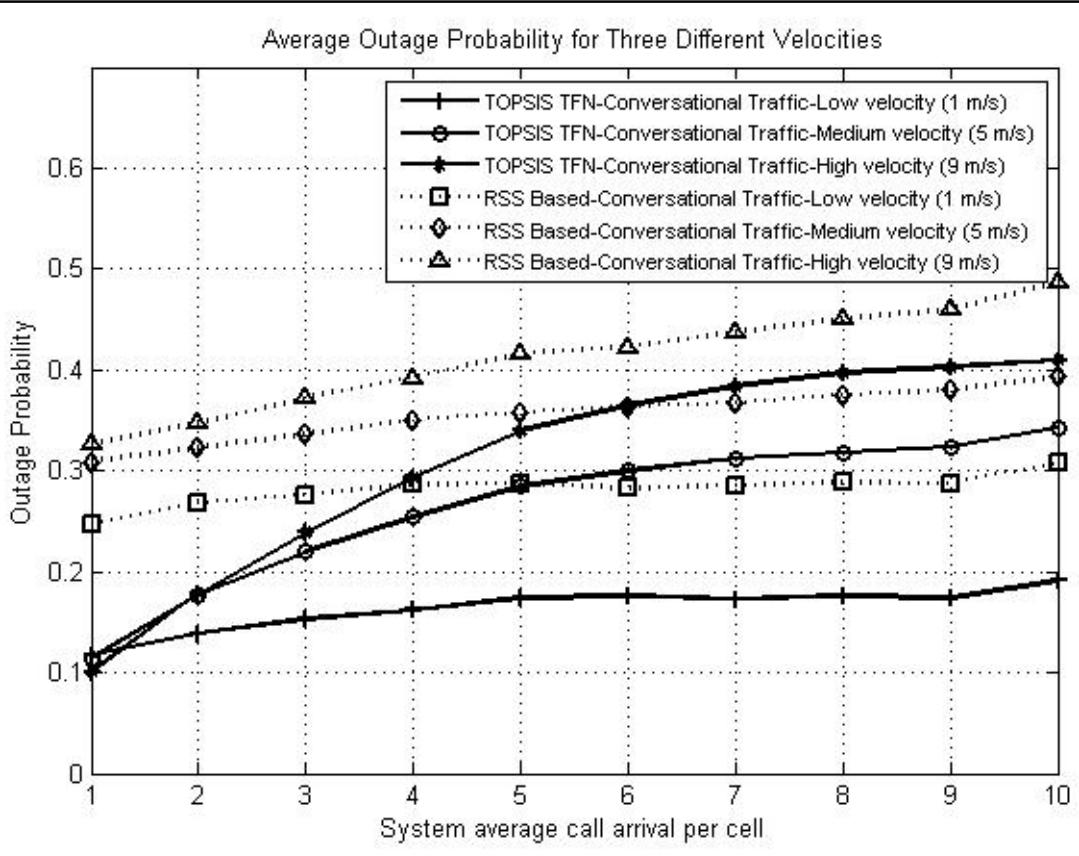

Figure 4.55: Average Outage Probability, TOPSIS, TFN, Conversational 


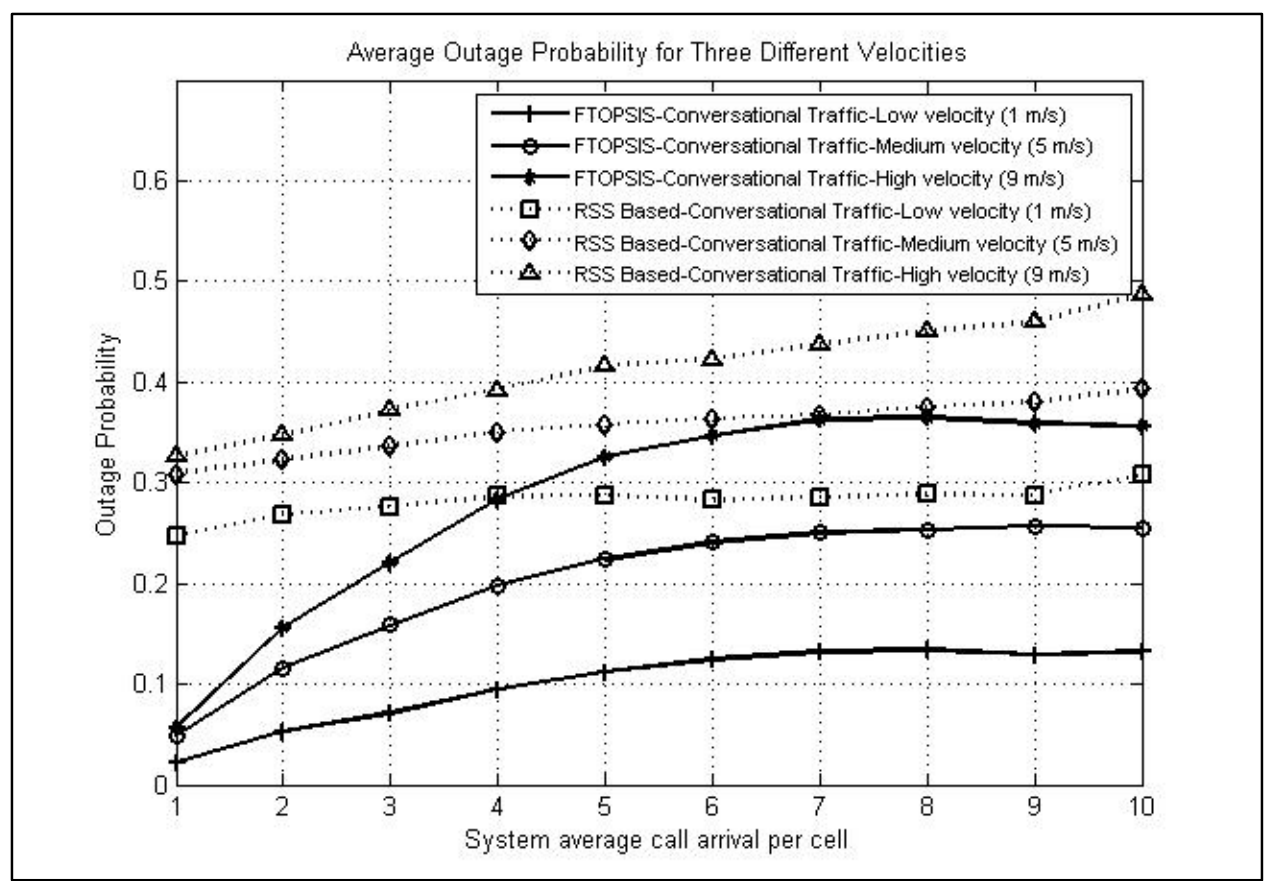

Figure 4.56: Average Outage Probability, FTOPSIS, Conversational

\subsubsection{AVERAGE OUTAGE PROBABILITY WITH FTOPSIS AND FVIKOR}

The FTOPSIS based network selection scheme shows significant performance improvement over traditional RSS-based and TOPSIS-based schemes. It can be observed from Figure 4.56, the average outage probability for MSs moving with slower speed with maximum number of calls per cell is around $13 \%$ as compared to RSS with $31 \%$, TOPSIS-FAHP with $18 \%$, and TOPSIS-TFN with 19\%, respectively. Similarly, at higher speeds and maximum system loading, the outage probability for FTOPSIS is around $35.5 \%$.

Fuzzy based FVIKOR network selection scheme performs better as compared with the traditional RSS and TOPSIS combined with AHP, FAHP, or TFN based schemes. For maximum number of average calls per cell, the average outage probabilities of $18 \%, 32 \%$, and $45 \%$ for slow, medium, and high moving MSs, respectively, can be 
observed from Figure 4.57. On the other hand, a comparison of Figure 4.56 with Figure 4.57 shows FTOPSIS providing superior performance with FVIKOR.

\subsubsection{AVERAGE HANDOFF BLOCKING PROBABILITY BASED ON TOPSIS}

As depicted in Figure 4.58, the TOPSIS-AHP based network selection scheme shows an overall reduced performance when compared with the reference algorithm. For slow moving MSs with a low number of average call arrivals per cell, the scheme performs marginally better than the RSS based reference algorithm. It can be observed from the figure, that for slow moving MSs, the TOPSIS-AHP scheme starts to diverge when the average number of calls per cell becomes greater than 3 . For medium and high speed MSs, this divergence takes place earlier as compared with slow speed MSs. On the other hand, a convergence can be seen for average call arrival rates between 7 and 10 .

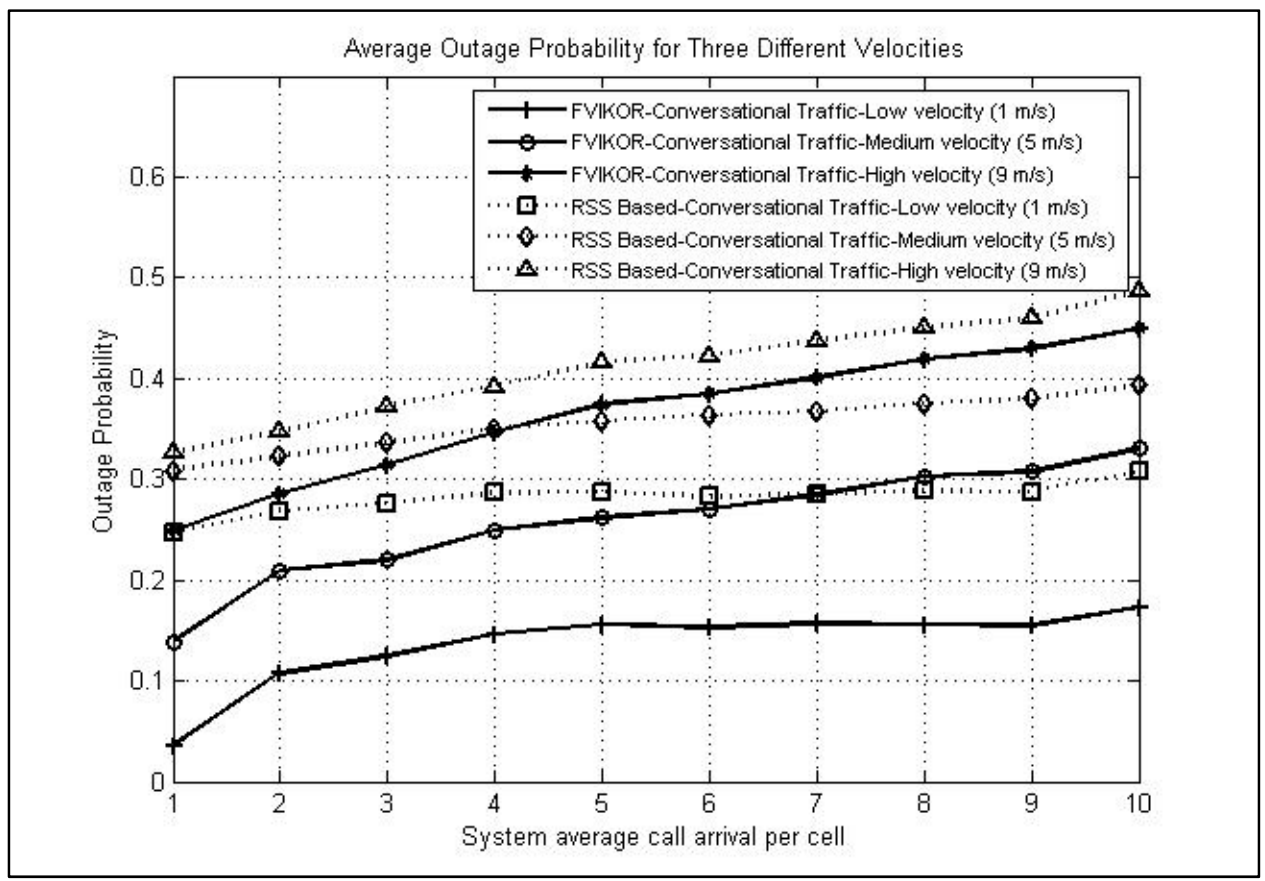

Figure 4.57: Average Outage Probability, FVIKOR, Conversational 


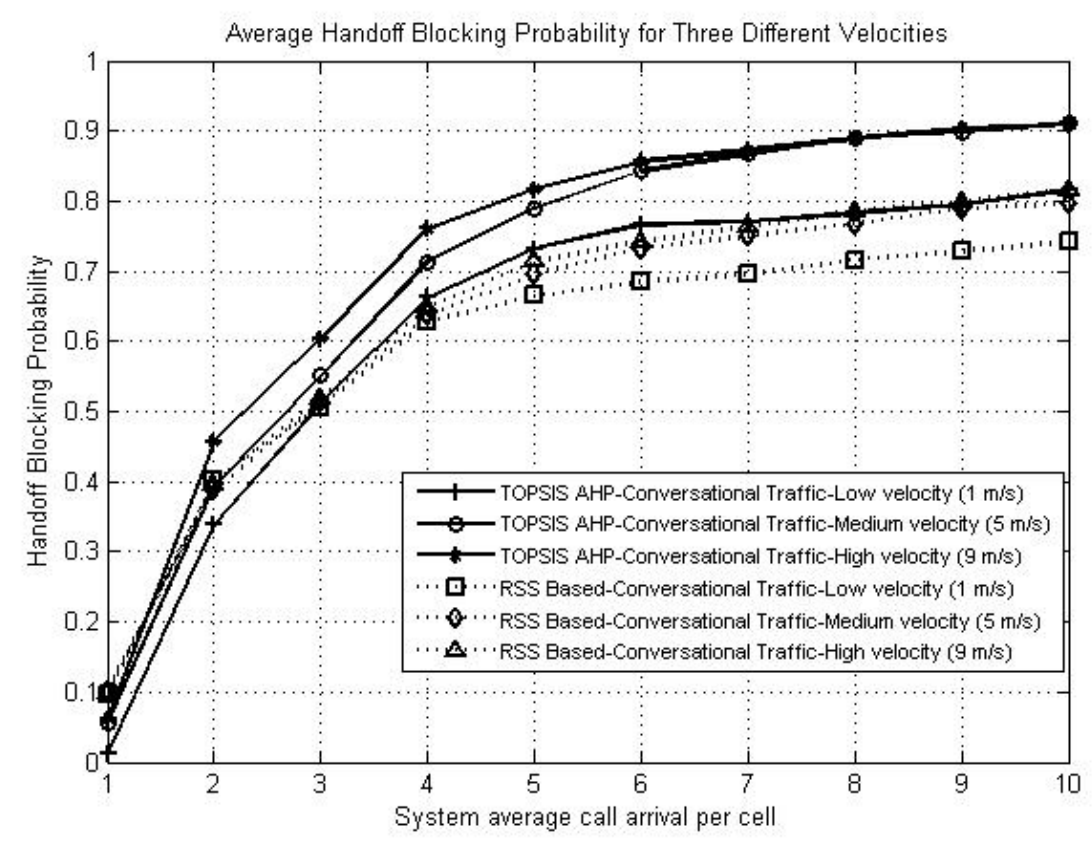

Figure 4.58: Average Handoff Blocking Probability based on TOPSIS-AHP

The performance of the TOPSIS-FAHP based scheme shows a little improvement when compared with TOPSIS-AHP scheme. It can be observed from Figure 4.59 that at MSs' speed of $1 \mathrm{~m} / \mathrm{s}$ the scheme provides a lower value of handoff blocking probability for average calls of 4 or less. On the other hand, with maximum average call arriving at each cell, the handoff blocking probability increases to approximately $92 \%$ for medium and higher speeds MSs.

TOPSIS-TFN scheme behaves similarly to TOPSIS-FAHP with a minor improvement for medium speed MSs with less numbers of system average call arrival per cell. This is shown in Figure 4.60. 


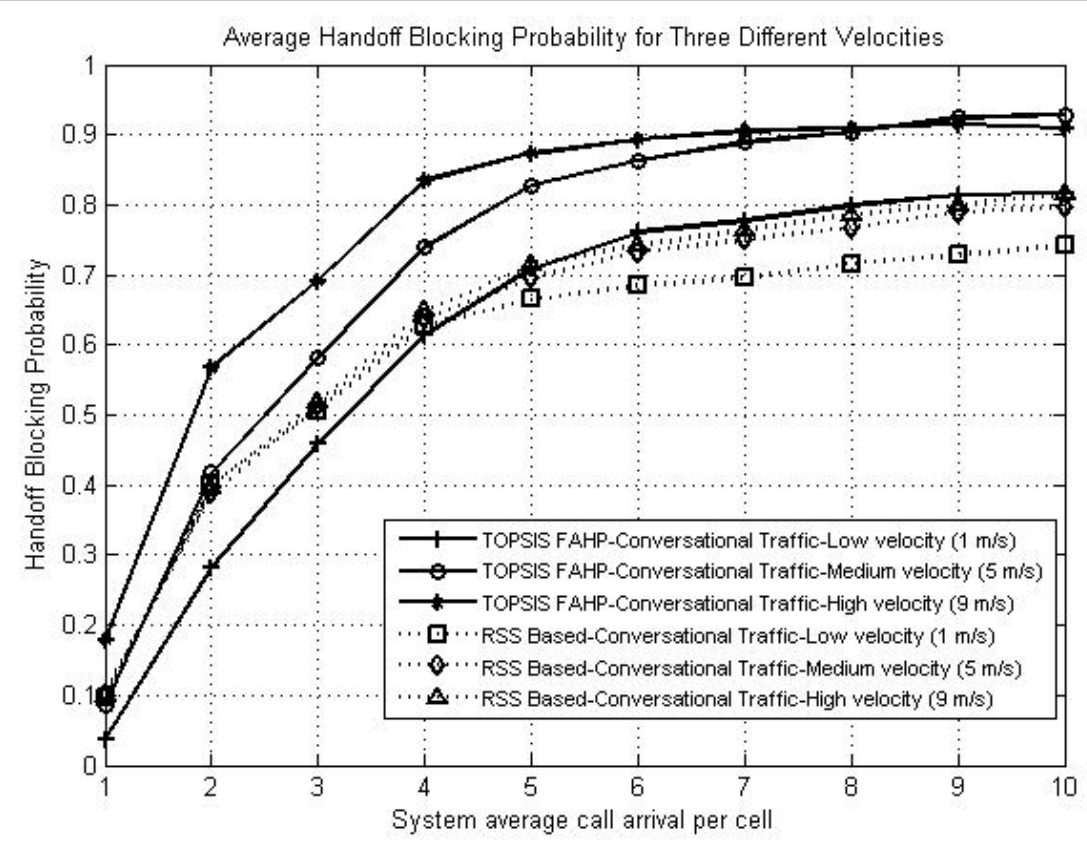

Figure 4.59: Average Handoff Blocking Probability based on TOPSIS-FAHP

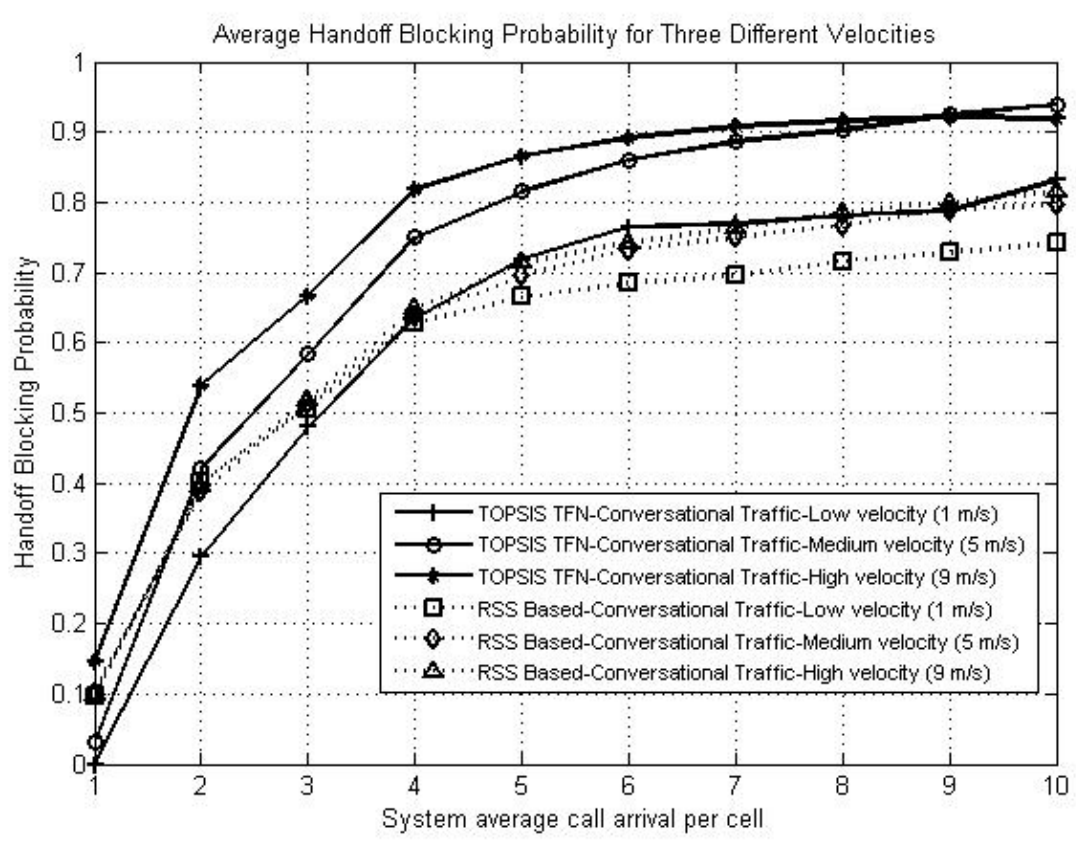

Figure 4.60: Average Handoff Blocking Probability based on TOPSIS-TFN 


\subsubsection{AVERAGE HANDOFF BLOCKING PROBABILITY BASED ON FTOPSIS AND FVIKOR}

Figure 4.61 shows the handoff blocking probability when FTOPSIS is used as the target network selection algorithm. It can be seen from this figure that FTOPSIS outperforms other schemes based on purely crisp data, or a hybrid of crisp and fuzzy data. For MSs moving at any speed, and for any number of average call arrivals per cell, FTOPSIS performs better than the above-mentioned schemes. For the maximum number of average calls arriving per cell, the handoff blocking probability is around $55 \%, 65 \%$, and $70 \%$, for slow, medium and high speed MSs, respectively. This can be compared against RSS based scheme with handoff blocking probabilities of $75 \%, 80 \%$, and $82 \%$, for slow, medium, and high speed MSs. This means that the FTOPSIS scheme makes better and intelligent decisions to find the best target network that can fulfill the end-user requirements.

The fuzzy based FVIKOR also shows improvements especially when there are increased numbers of average system calls per cell. For mobile stations moving with medium or higher speeds with an average number of 4 calls or less, the reference algorithm performs better than FVIKOR. Nevertheless, FVIKOR shows improvements for average calls of 5 and more per cell. It is important to note that FVIKOR does not

provide the same performance as FTOPSIS, when the average handoff blocking probability at maximum system loading for MSs with average speeds of $9 \mathrm{~m} / \mathrm{s}$ is around $70 \%$ as compared with FVIKOR, where the blocking probability is around $75 \%$. 


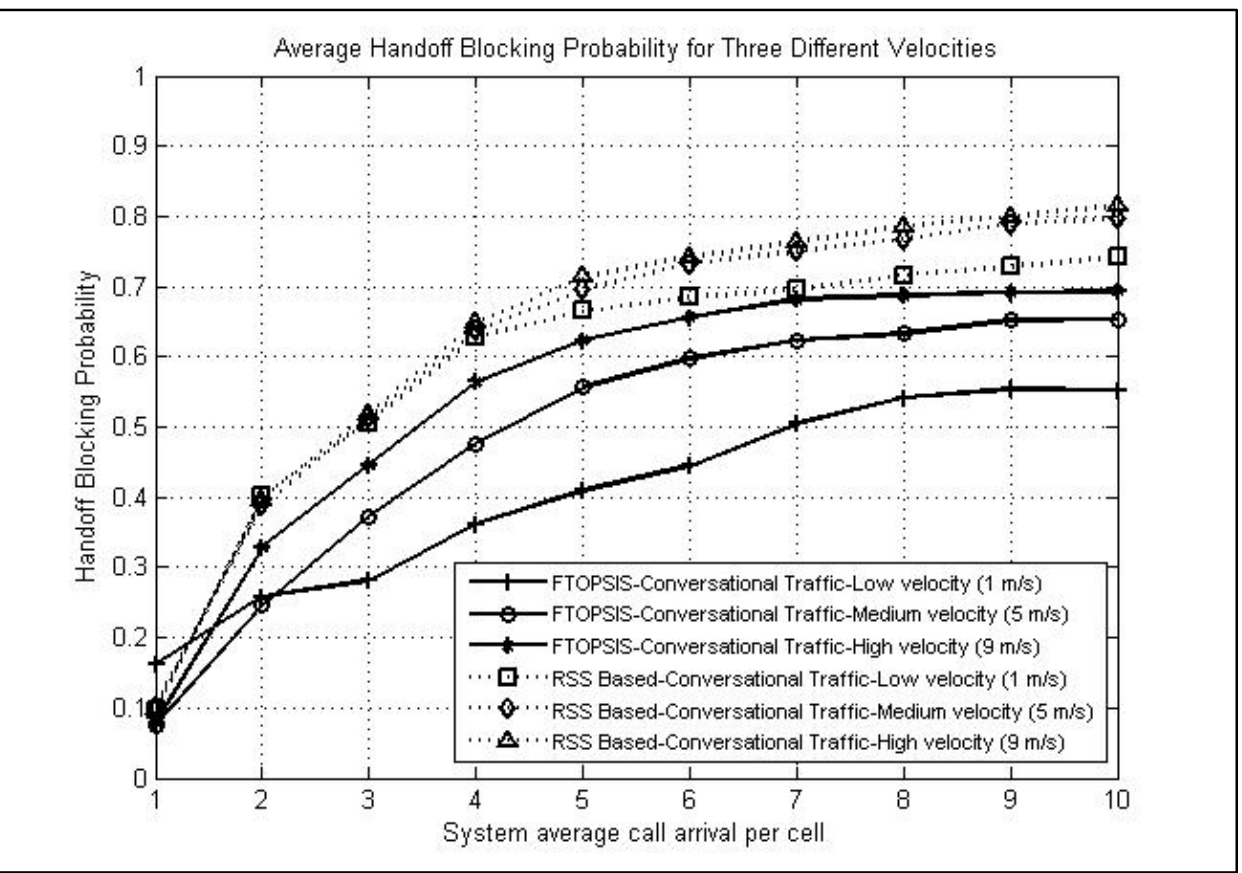

Figure 4.61: Average Handoff Blocking Probability based on FTOPSIS

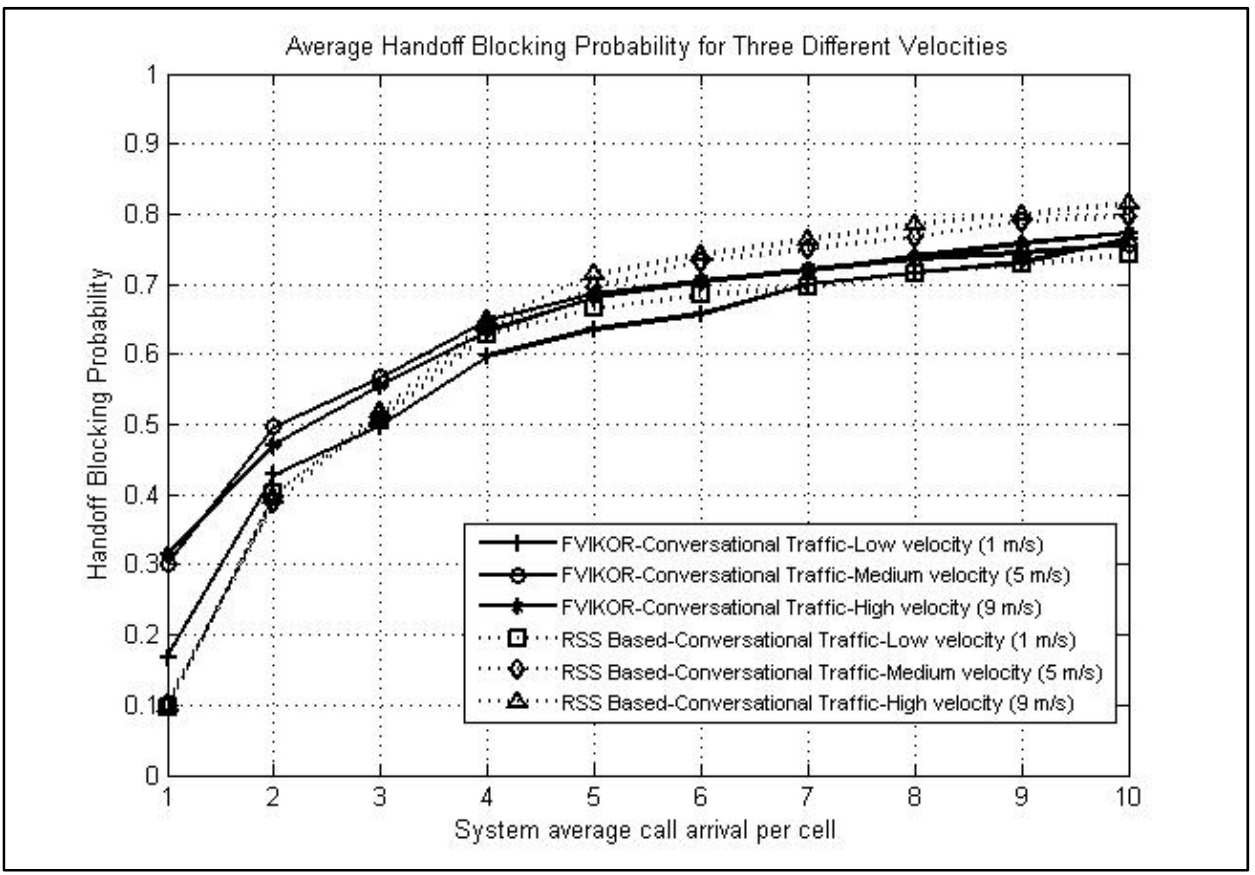

Figure 4.62: Average Handoff Blocking Probability based on FVIKOR 


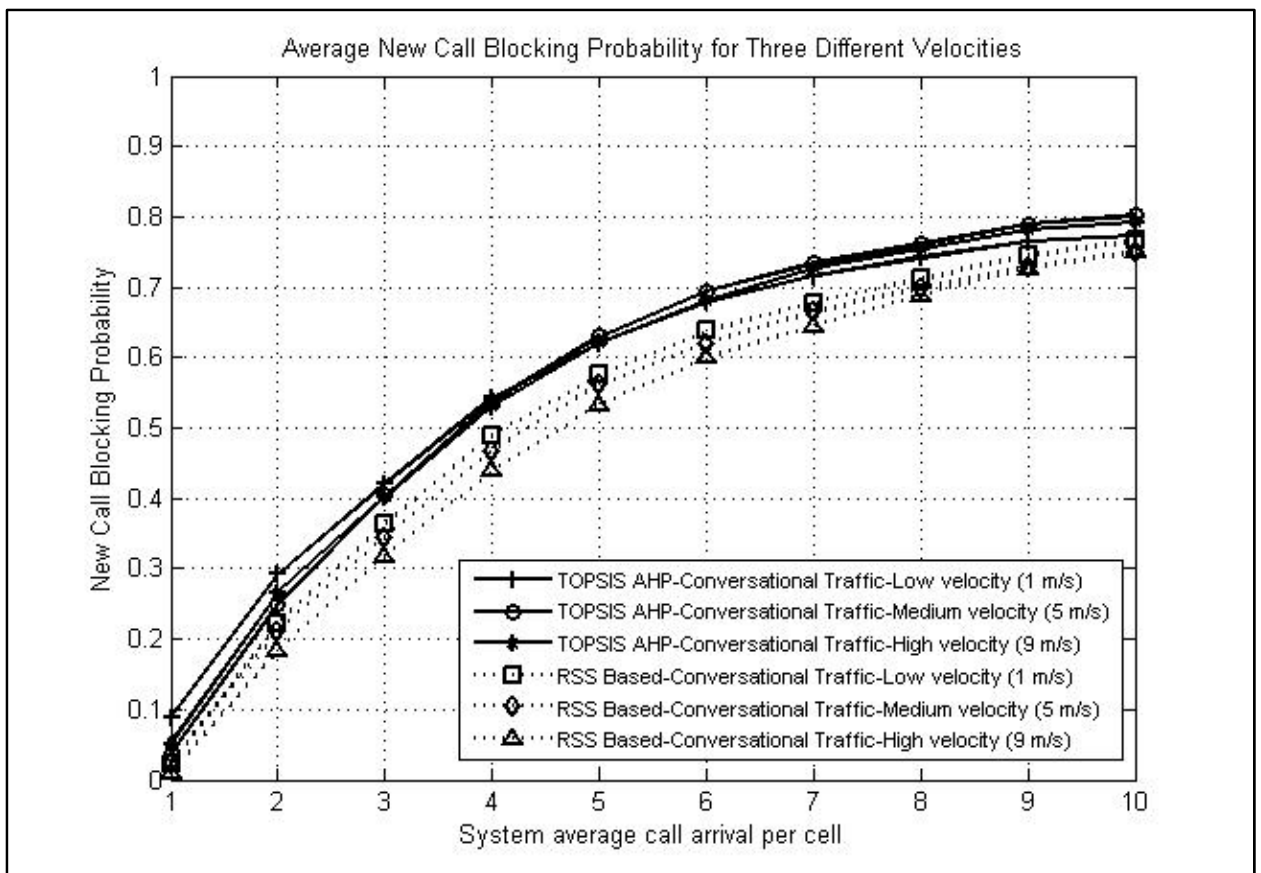

Figure 4.63: Average New Call Blocking Probability based on TOPSIS-AHP

\subsubsection{AVERAGE NEW CALL BLOCKING PROBABILITY BASED ON TOPSIS}

The average new call blocking probability for TOPSIS based schemes is presented in Figures 4.63-4.65. Just like average handoff blocking probability, a similar trend can be observed from these figures. The traditional RSS based scheme is able to provide a better performance as compared with all TOPSIS based schemes. But unlike Figures 4.58-4.60 where the average handoff blocking probability at maximum system load of 10 calls per cell is above $90 \%$, the probability of new call blocking is below $80 \%$. 


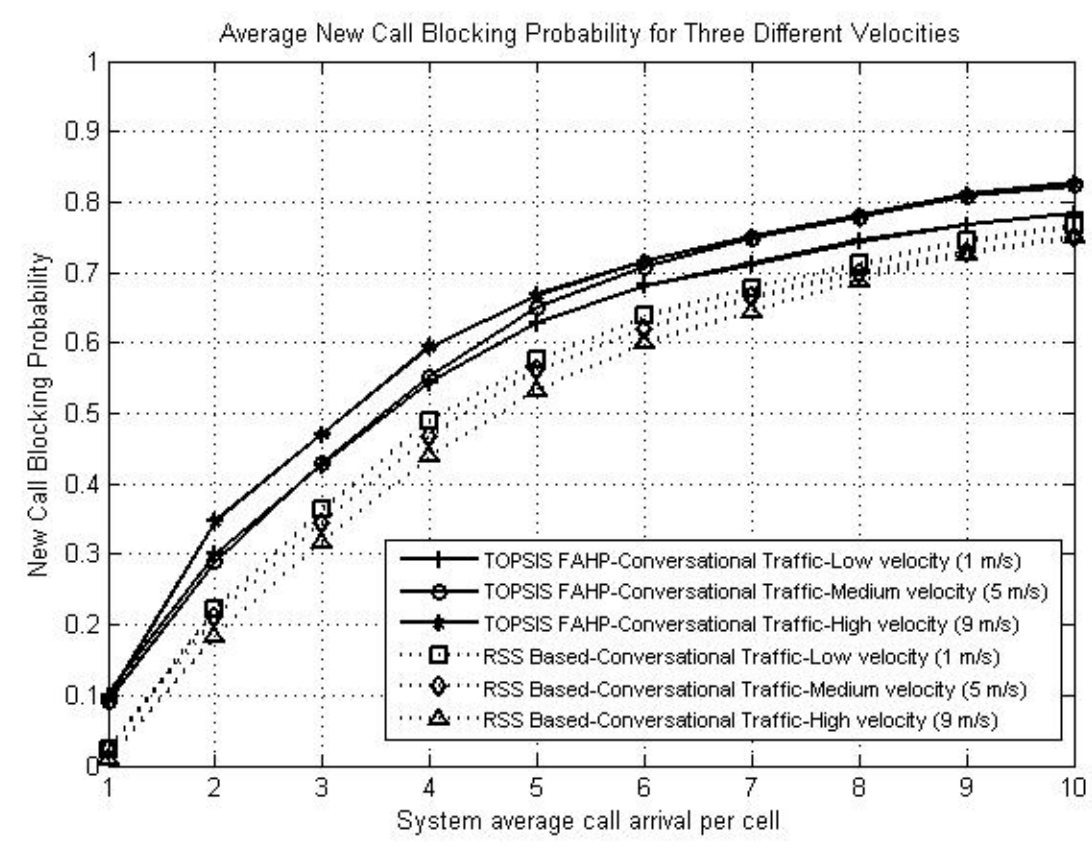

Figure 4.64: Average New Call Blocking Probability based on TOPSIS-FAHP

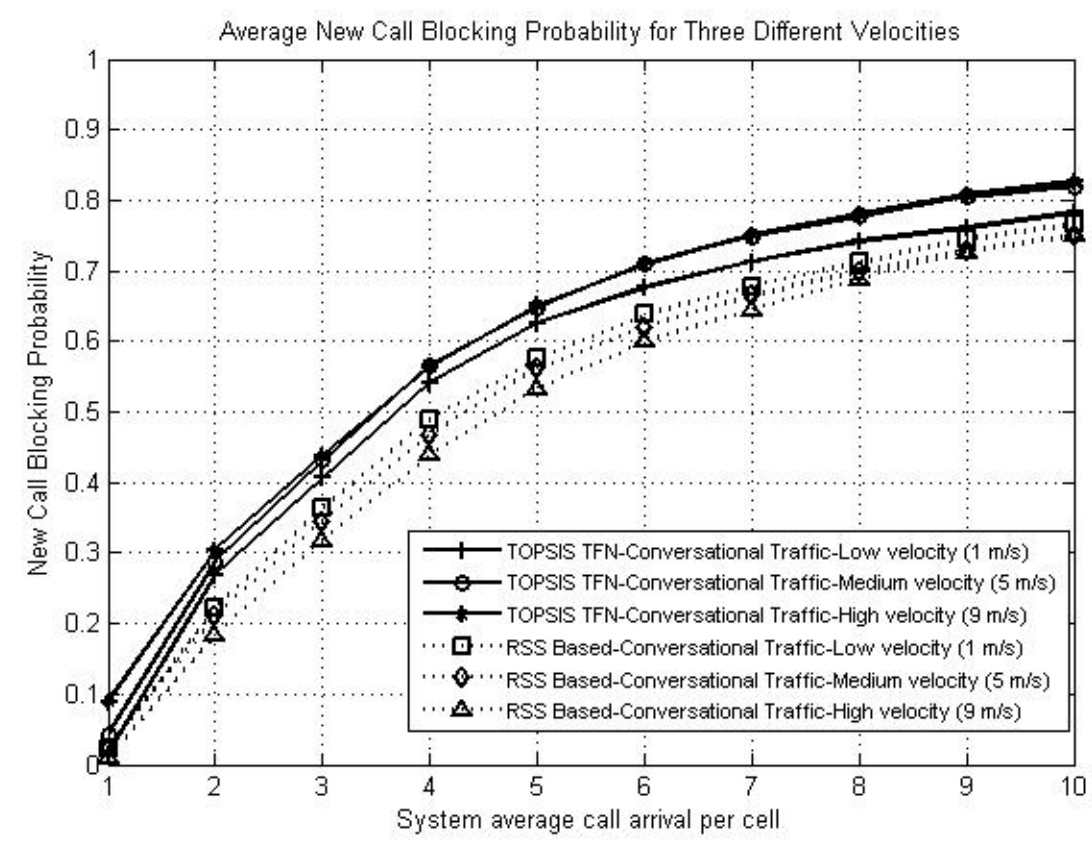

Figure 4.65: Average New Call Blocking Probability based on TOPSIS-TFN 


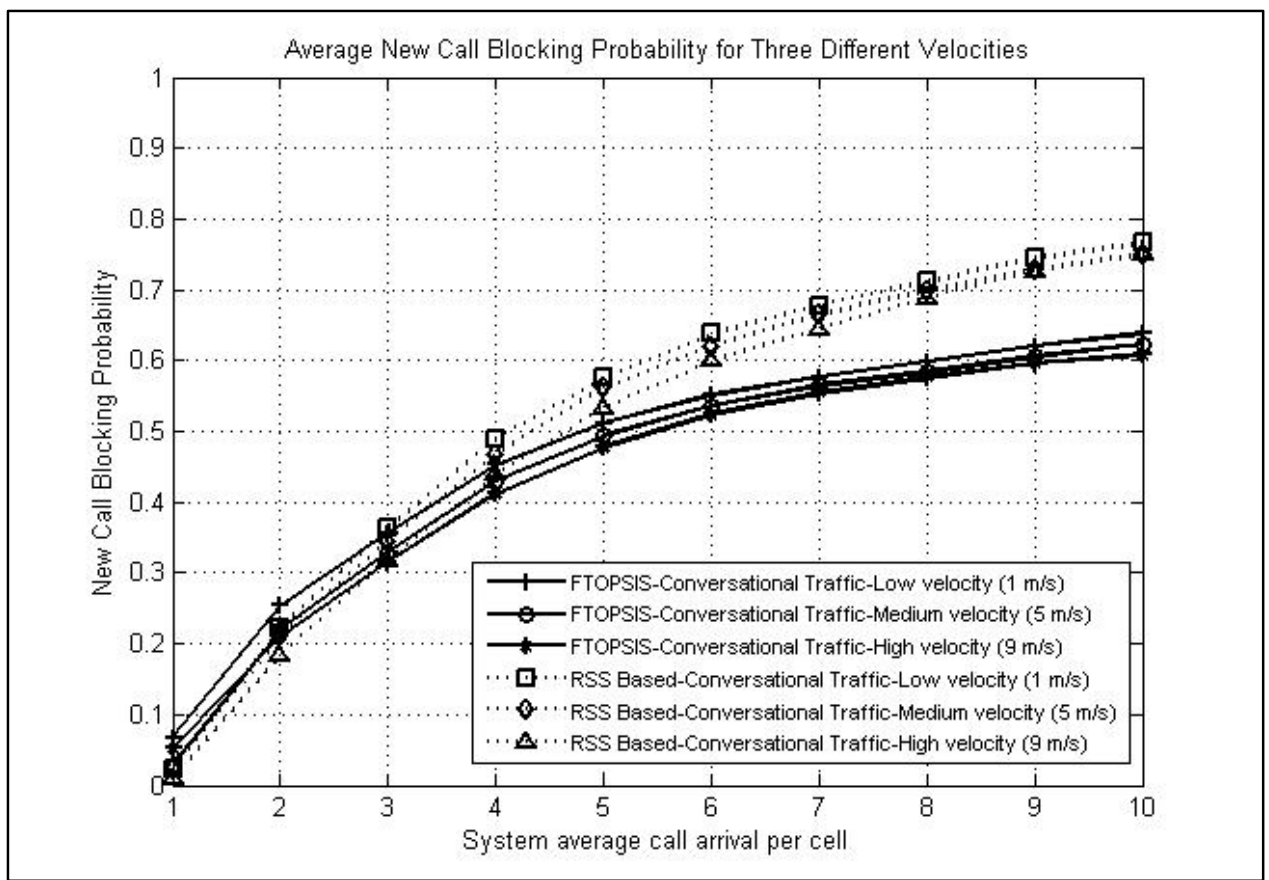

Figure 4.66: Average New Call Blocking Probability based on FTOPSIS

\subsubsection{AVERAGE NEW CALL BLOCKING PROBABILITY BASED ON FTOPSIS} AND FVIKOR

Figure 4.66 shows the new call blocking probability based on FTOPSIS ranking module. As discussed earlier, the evaluation of VHITS target network selection module can be done based on this metric. This figure clearly depicts an overall enhanced performance, especially for system-loading with an average call arrival rate of 3 and more. For a very busy system, FTOPSIS produces a new call blocking probability of less than $65 \%$ for MSs moving with any speed. On the other hand, FVIKOR, depicted in Figure 4.67, shows promising results only for an average call arrival rate of 8 and more per cell. Again, FTOPSIS shows better performance when compared with the other demonstrated schemes. 


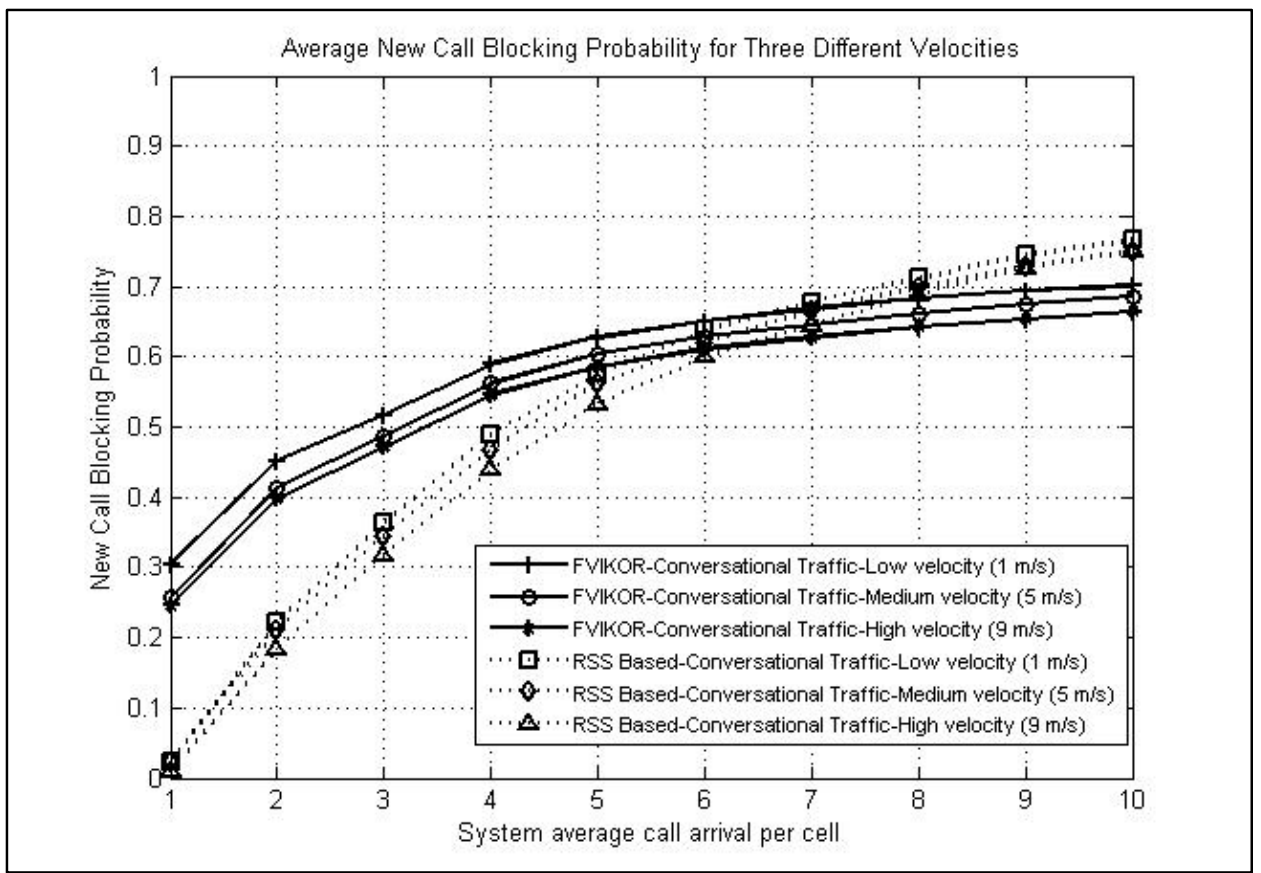

Figure 4.67: Average New Call Blocking Probability based on FVIKOR

\subsubsection{AVERAGE HANDOFF RATE BASED ON TOPSIS}

Figures 4.68-4.70 depict the average number of handoff rates that the system has performed during an on-going call/session, using TOPSIS with AHP, FAHP, and TFN weighting schemes. It is obvious from these figures that the average handoff rate of the VHITS scheme utilizing TOPSIS with any weighting scheme is far better than the RSS based reference algorithm. With MSs moving with high speeds and with system at maximum load, the average handoff rate is approximately $50 \%$ for TOPSIS-AHP scheme. On the other hand, when TOPSIS is combined with FAHP or TFN weighting mechanisms, an improvement of $24 \%$ over RSS based algorithm can be noted for higher speed MSs accessing system with higher number of arrival calls per cell. 


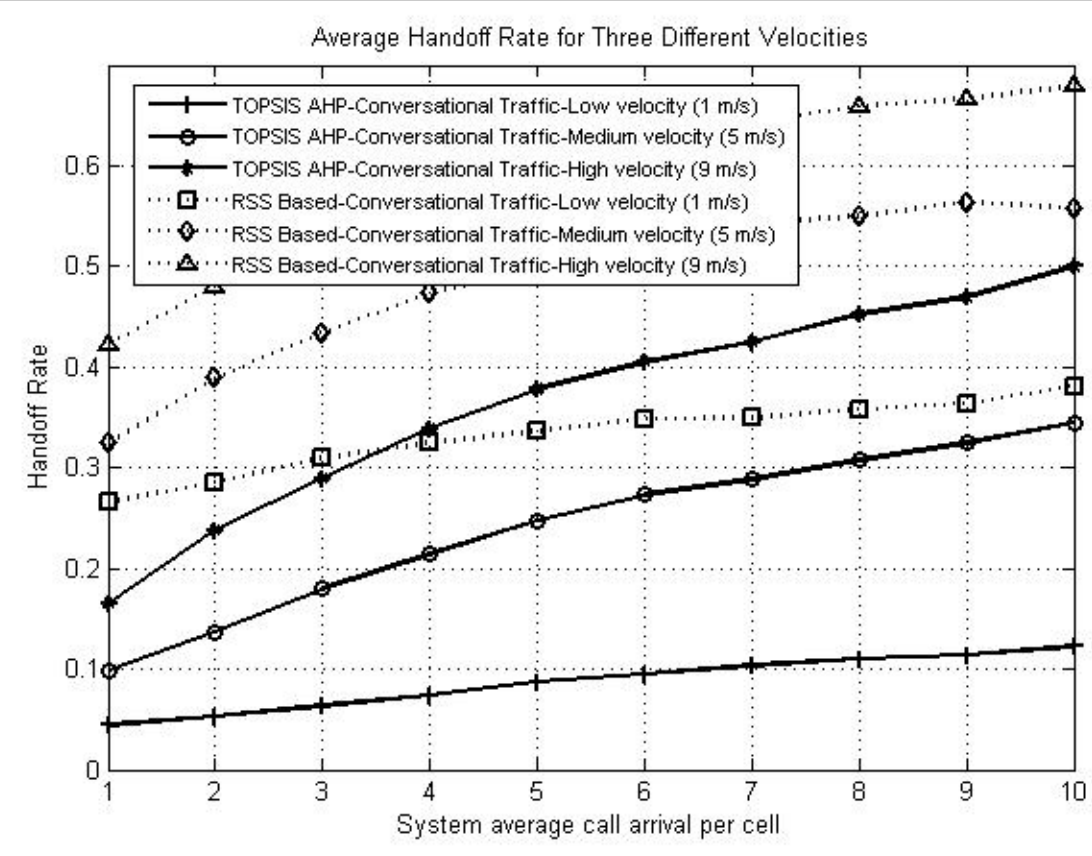

Figure 4.68: Average Handoff Rate based on TOPSIS-AHP

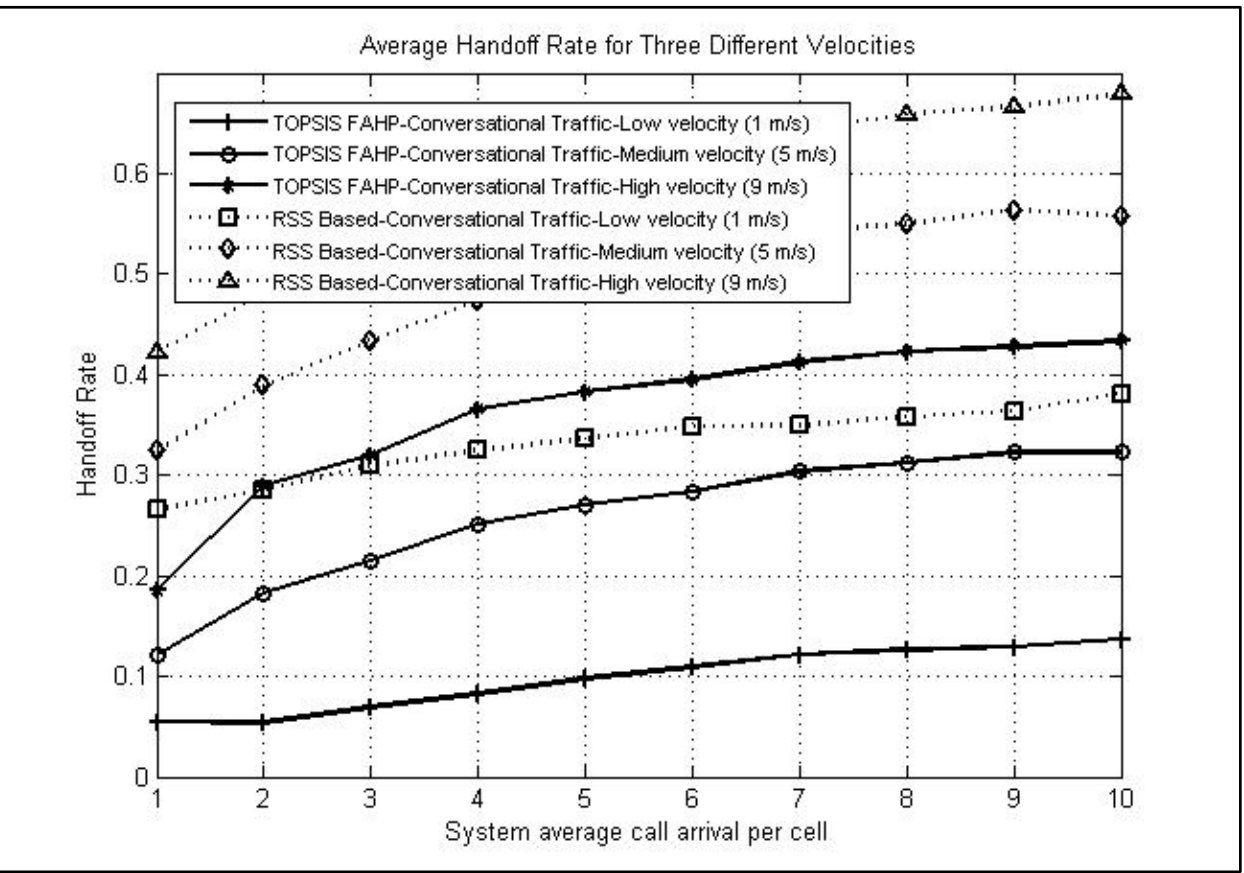

Figure 4.69: Average Handoff Rate based on TOPSIS-FAHP 


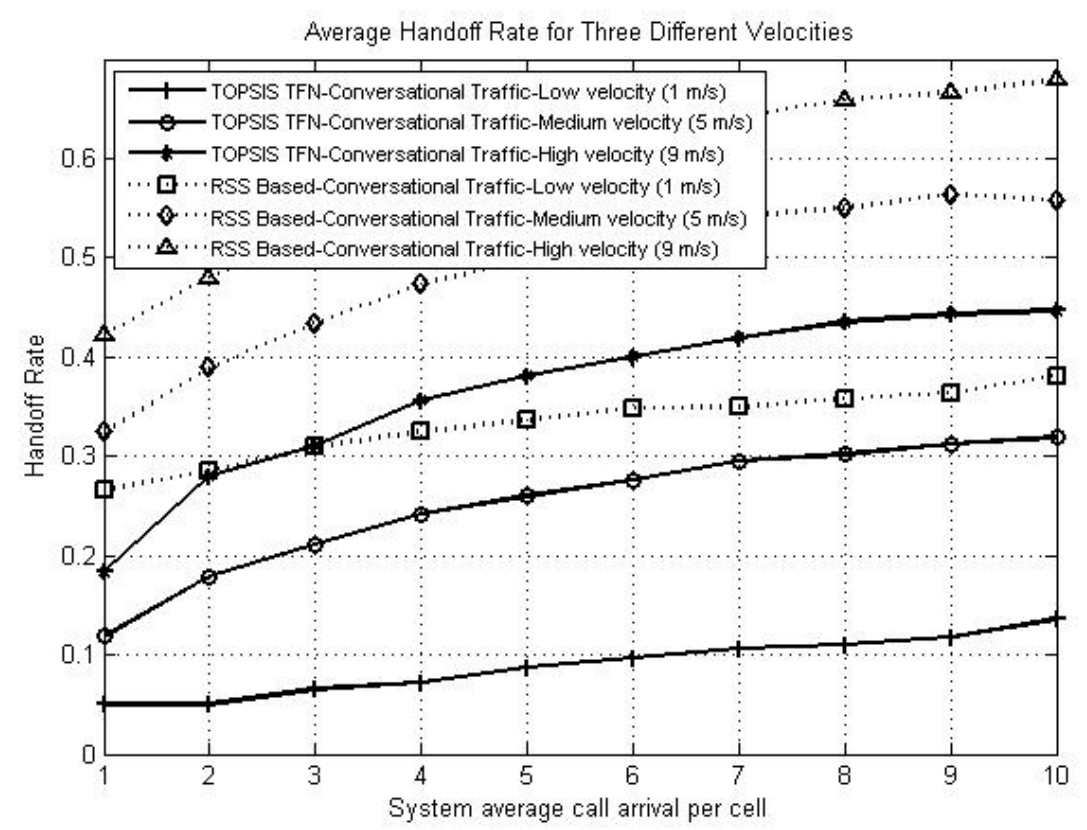

Figure 4.70: Average Handoff Rate based on TOPSIS-TFN

This overall performance gain, in terms of average number of handoffs, is due to the design of VHITS necessity estimation module that incorporates parallel FLCs and takes advantage of GPT with a moving average filter to predict RSS based on the measured samples from available networks; furthermore, this results in reduced and intelligent handoffs to the best available target networks. Due to the intelligence of the VHITS necessity estimation module, the "ping-pong effect" is also reduced significantly.

\subsubsection{AVERAGE HANDOFF RATE BASED ON FTOPSIS AND FVIKOR}

The average handoff rate for VHITS utilizing FTOPSIS and FVIKOR is presented in Figure 4.71 and Figure 4.72, respectively. Once again, these fuzzy-data based schemes demonstrate a superior performance when compared against the reference algorithm and the different TOPSIS combinations discussed in the above paragraphs. As can be seen from these figures, the performance of FTOPSIS is better than FVIKOR. An 
improvement of $32 \%$ and $7 \%$ can be seen for FTOPSIS when compared to RSS-based and FVIKOR schemes, respectively. These handoff rates are calculated for average call arrival rate of 10 per cell and with MSs' speed of $9 \mathrm{~m} / \mathrm{s}$. This improvement over traditional scheme shows that the VHITS scheme is performing handoff necessity estimation and target selection mechanism in a more intelligent and efficient manner.

\subsubsection{PERCENTAGE OF NETWORK CONNECTIONS BASED ON AVERAGE} SYSTEM CALLS-TOPSIS

This and the following sections discuss the overall percentages of network connections that the proposed scheme assigns to the three types of available networks based on the Conversational traffic class. The effect of different speeds of MSs is observed for three different average arrival rates of 1,5 , and 10 calls per cell. RSS based selection mechanism that is used as reference algorithm is also provided. Note that these percentages are calculated after the completion of the entire simulation to observe and discover some interesting trends based on the overall state of the system.

Figures 4.73-4.81 show the percentages of network connections based on TOPSIS with AHP, FAHP, and TFN weighting schemes. A common trend observed from these figures is that WWAN is consistently given higher preference as compared with WMAN, and WLAN. This is true for any mobile speed and any number of average system calls per cell. WMAN and WLAN are given second and third preferences, respectively. As explained earlier Conversational traffic class requires a low value of delay and jitter, and according to the chosen parameters listed in Table 4.11, WWAN provides the lowest values for these attributes, followed by WMAN and WLAN. 


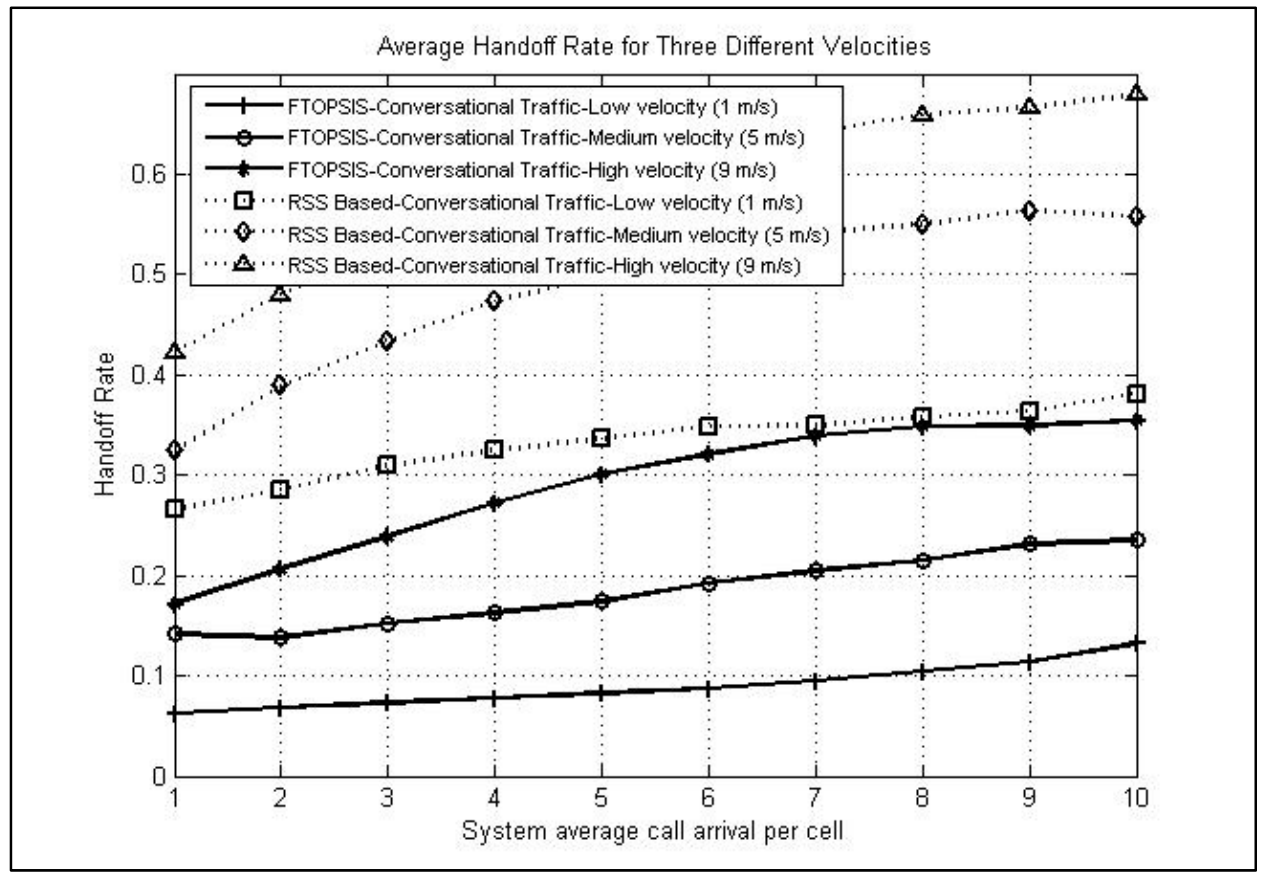

Figure 4.71: Average Handoff Rate based on FTOPSIS

As the speed of the MS increases from $1 \mathrm{~m} / \mathrm{s}$ to $9 \mathrm{~m} / \mathrm{s}$, the percentage of network connections to WWAN also increases for all three system traffic loadings. This should be the case, as the proposed scheme prefers WWAN for higher speed MSs. For example, for an average call arrival rate of 1 , it can be observed that the percentage of connectivity towards WWAN based on TOPSIS-AHP, increases from $70 \%$ to $75 \%$ for average MSs' speeds of $1 \mathrm{~m} / \mathrm{s}$ and $9 \mathrm{~m} / \mathrm{s}$, respectively. 


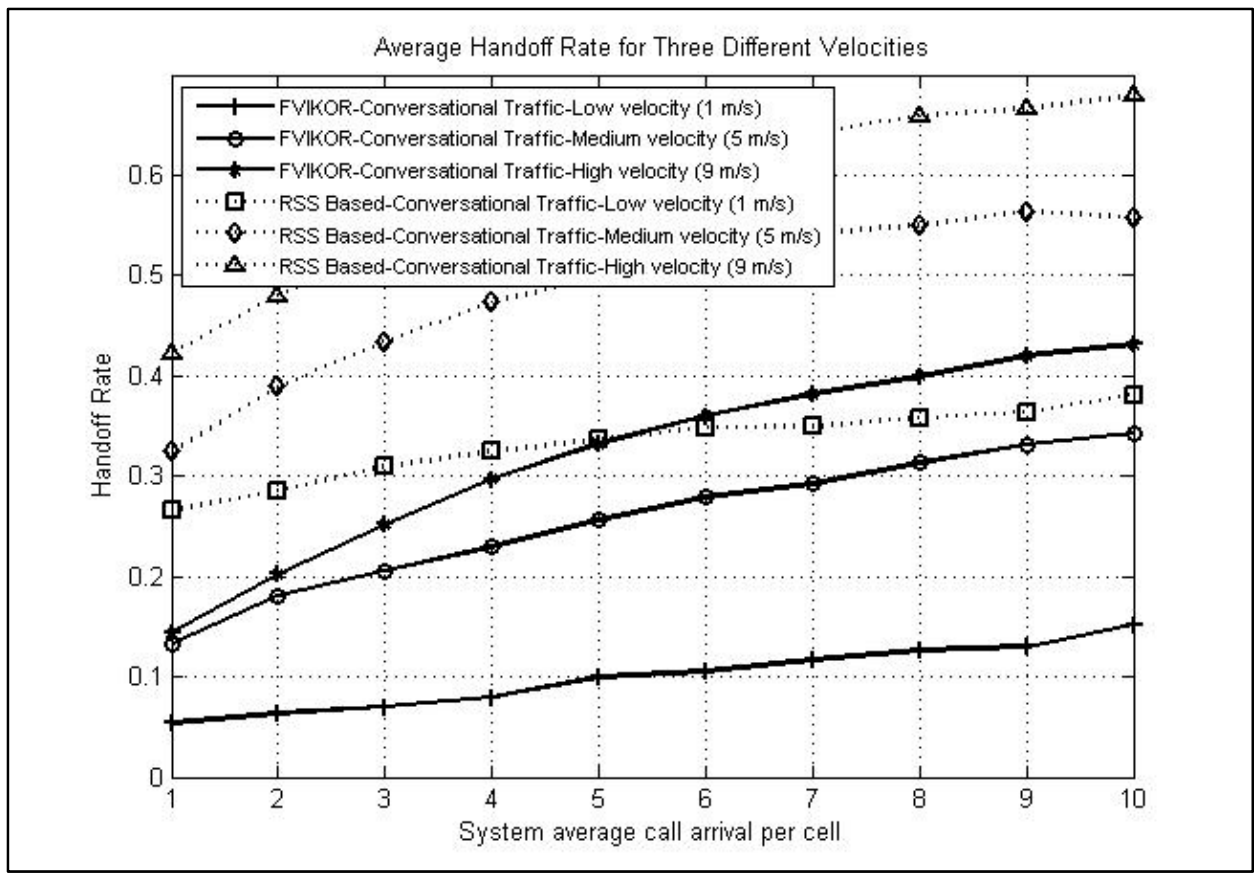

Figure 4.72: Average Handoff Rate based on FVIKOR

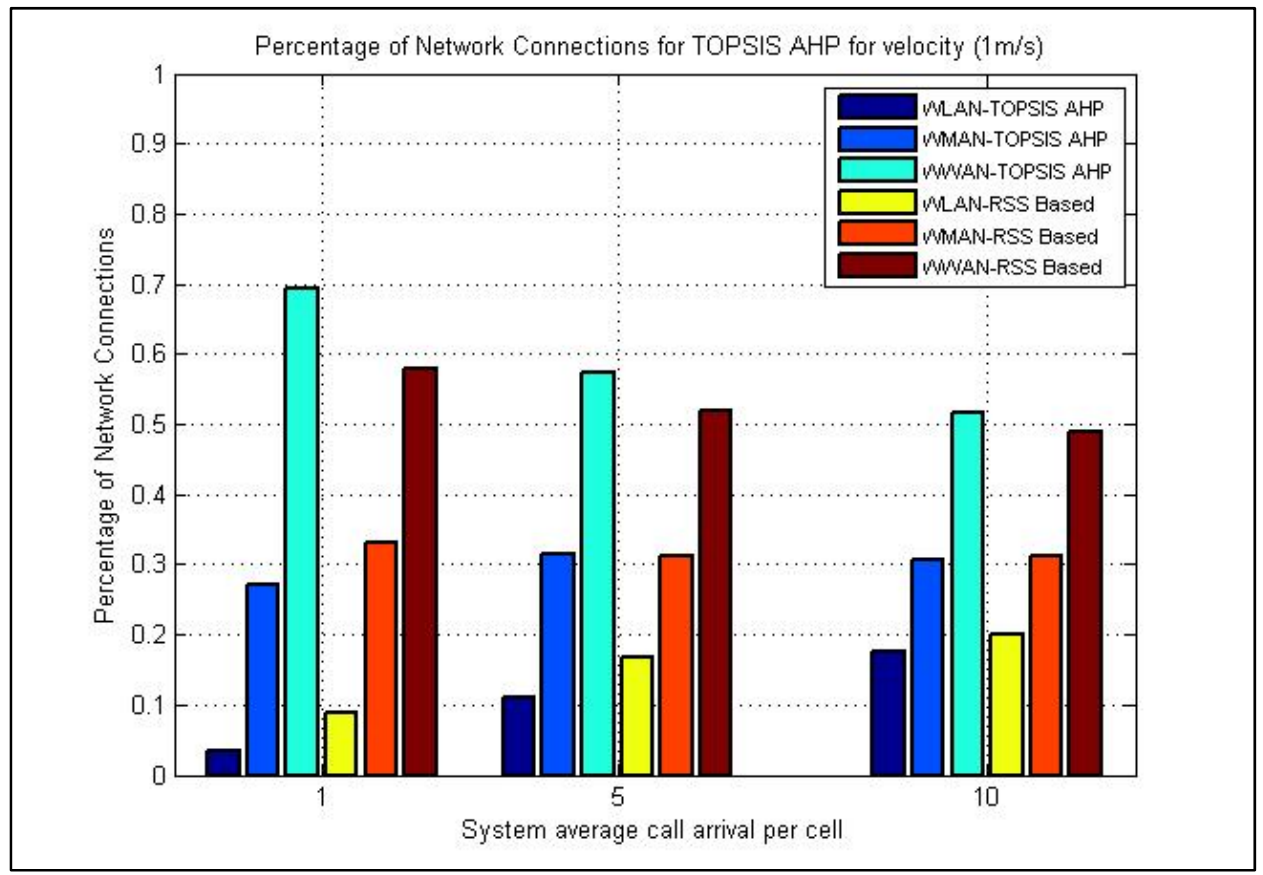

Figure 4.73: Percentage of NW-Connection per Number of Average Calls, TOPSIS-AHP, Velocity $=1 \mathrm{~m} / \mathrm{s}$ 
A similar pattern can be observed when the average number of calls increases from 1 to 10. At an average call arrival rate of 10, a distribution of connections among the three available networks can be observed. Based on the characteristics of the Conversational traffic class, the VHITS scheme still assigns more calls to WWAN as it currently offers a better overall QoS for the Conversational traffic class. For example, Figure 4.73 shows approximately $51 \%, 31 \%$, and $18 \%$ of the network connection assignments to WWAN, WMAN, and WLAN, respectively, at a system average call arrival rate of 10 and with mobiles moving with an average speed of $1 \mathrm{~m} / \mathrm{s}$. As discussed above, an increase in speed causes the proposed scheme to assign more calls to WWAN due to its larger coverage. For the same case, this can be observed from Figure 4.75 where these percentages have changed to $56 \%, 32 \%$, and $12 \%$, for WWAN, WMAN, and WLAN, respectively.

A comparison of the proposed scheme can be done against the RSS based reference algorithm, where for all the above-mentioned cases the proposed scheme performs better by assigning more calls/sessions to WWAN. For example, Figure 4.75 shows that the proposed scheme assigns approximately $75 \%$ of the connections to WWAN for a system average call arrival rate of 1 , as compared with $50 \%$ calls assigned by RSS based scheme. For an average call arrival rate of 10, the proposed scheme still outperforms RSS by a difference of approximately $9 \%$.

TOPSIS with FAHP and TFN based mechanisms offer similar trends as discussed above. At slower speeds, the call assignments based on TOPSIS-FAHP favor WLAN and WMAN by very small percentages. This can be observed from Figure 4.76 where the percentage of WMAN and WLAN has increased by $1 \%$ as compared with Figure 4.73 , 
when the system arrival rate is 1 . For medium and high speeds MSs, the TOPSIS-FAHP, as shown in Figures 4.77-4.78, assigns higher priority to WWAN than the TOPSIS-AHP scheme. This is due to the fact that FAHP utilizes fuzzy data for calculating system weights.

A comparison of Figures 4.76 and 4.79 show that at slower speeds, the performances of TOPSIS-FAHP and TOPSIS-TFN are almost the same. For MSs with medium speeds, TOPSIS with TFN assigns more call to WMAN as compared with WLAN. This can be observed from Figure 4.80. A minor performance difference between TOPSIS-FAHP and TOPSIS-TFN, at medium and higher arrival call rates, can be observed from Figure 4.81 .

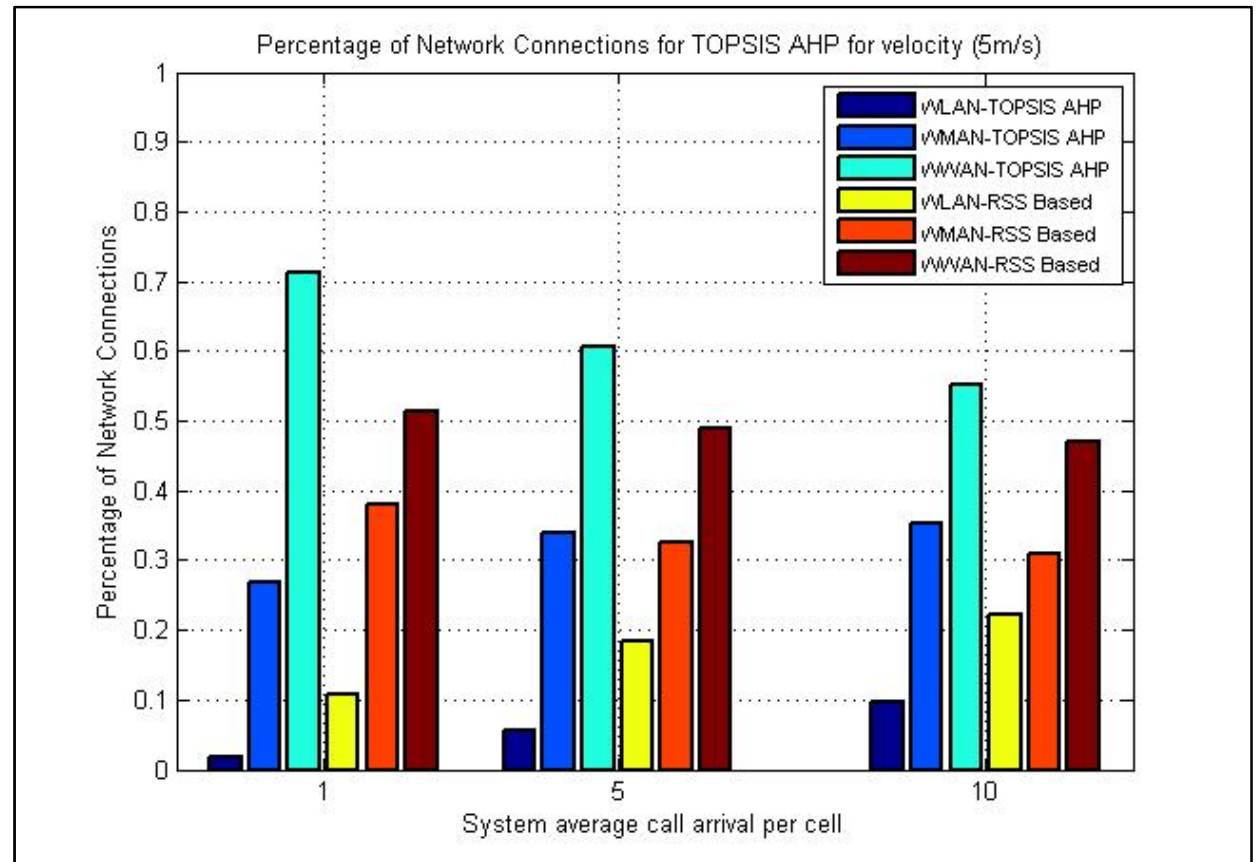

Figure 4.74: Percentage of NW-Connection per Number of Average Calls, TOPSIS-AHP, Velocity $=5 \mathrm{~m} / \mathrm{s}$ 


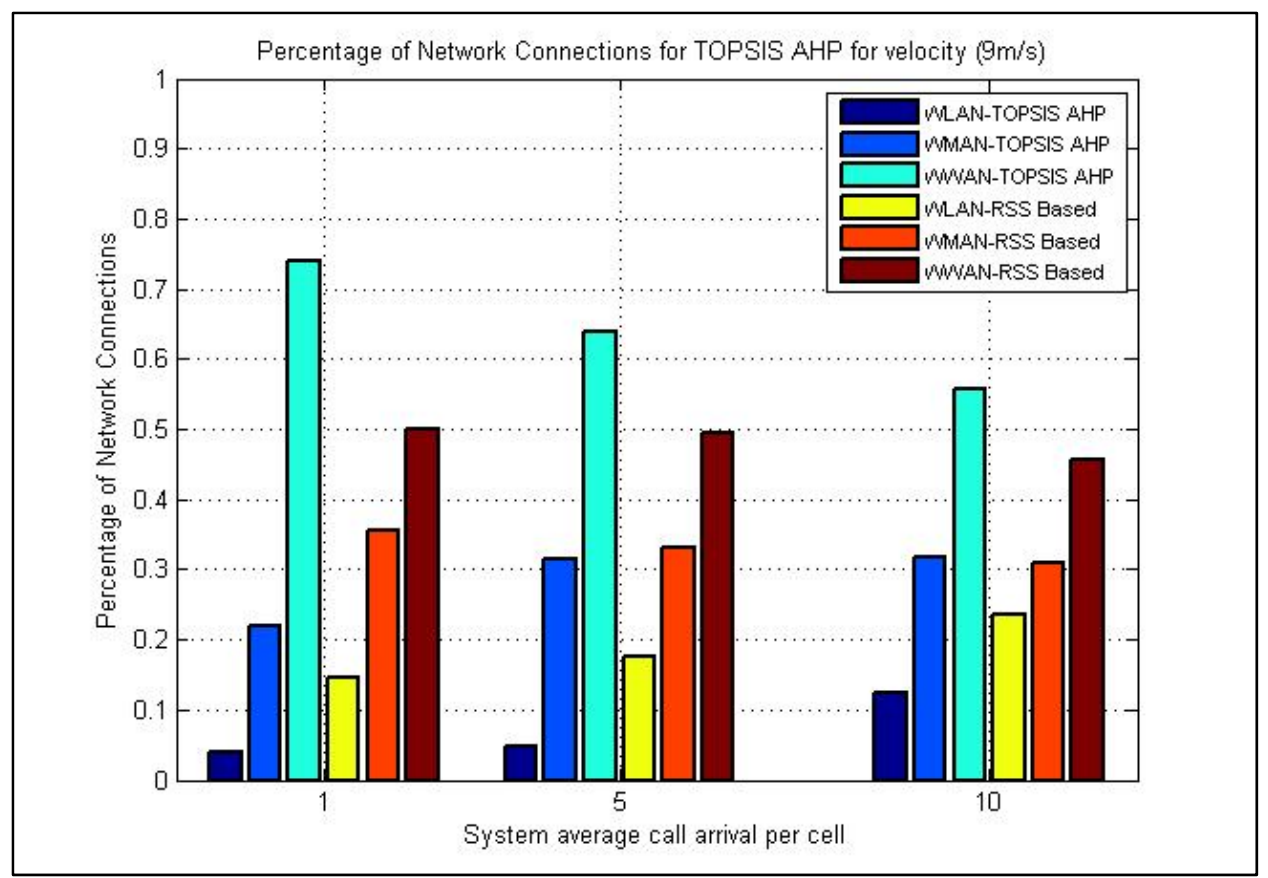

Figure 4.75: Percentage of NW-Connections per Number of Average Calls, TOPSIS-AHP, Velocity $=9 \mathrm{~m} / \mathrm{s}$

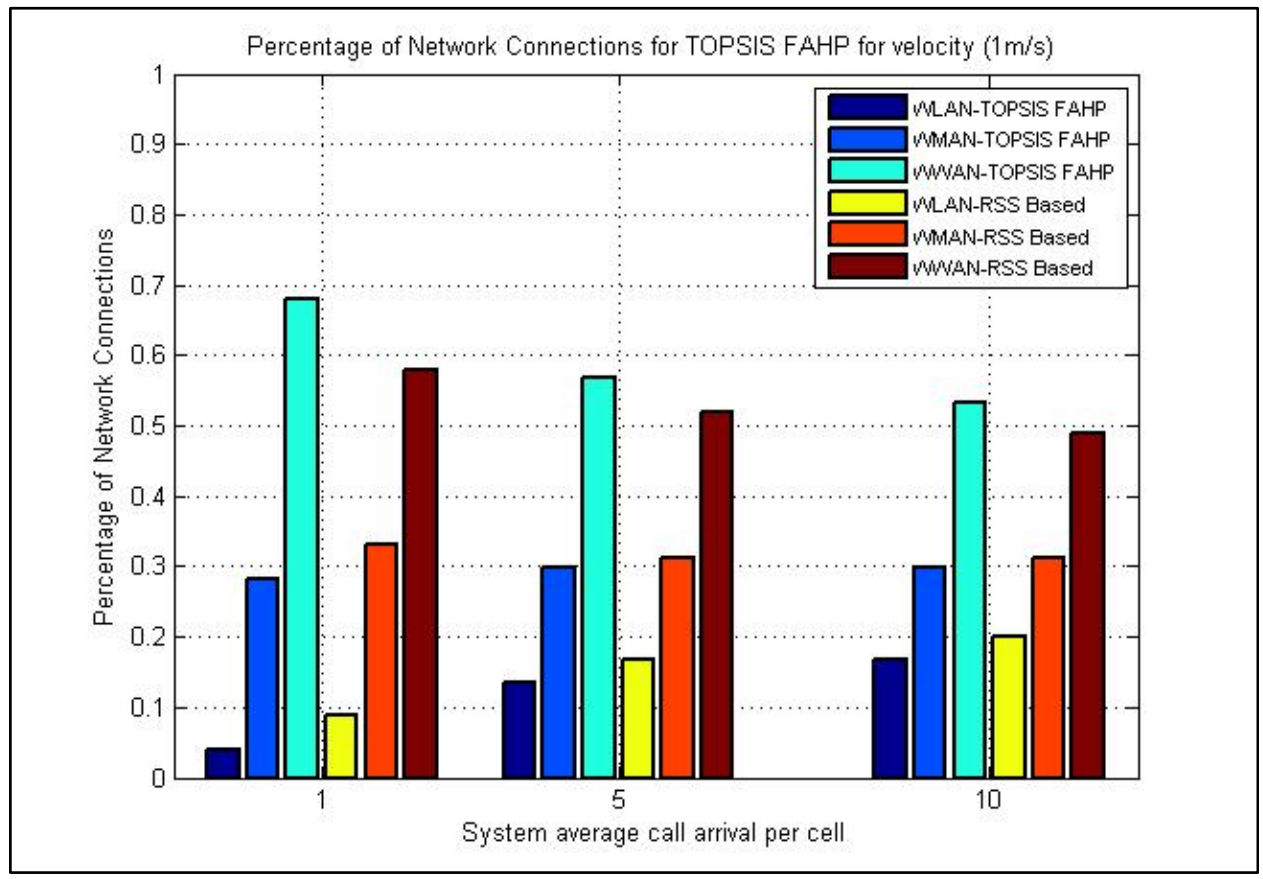

Figure 4.76: Percentage of NW-Connections per Number of Average Calls, TOPSIS-FAHP, Velocity $=1 \mathrm{~m} / \mathrm{s}$ 


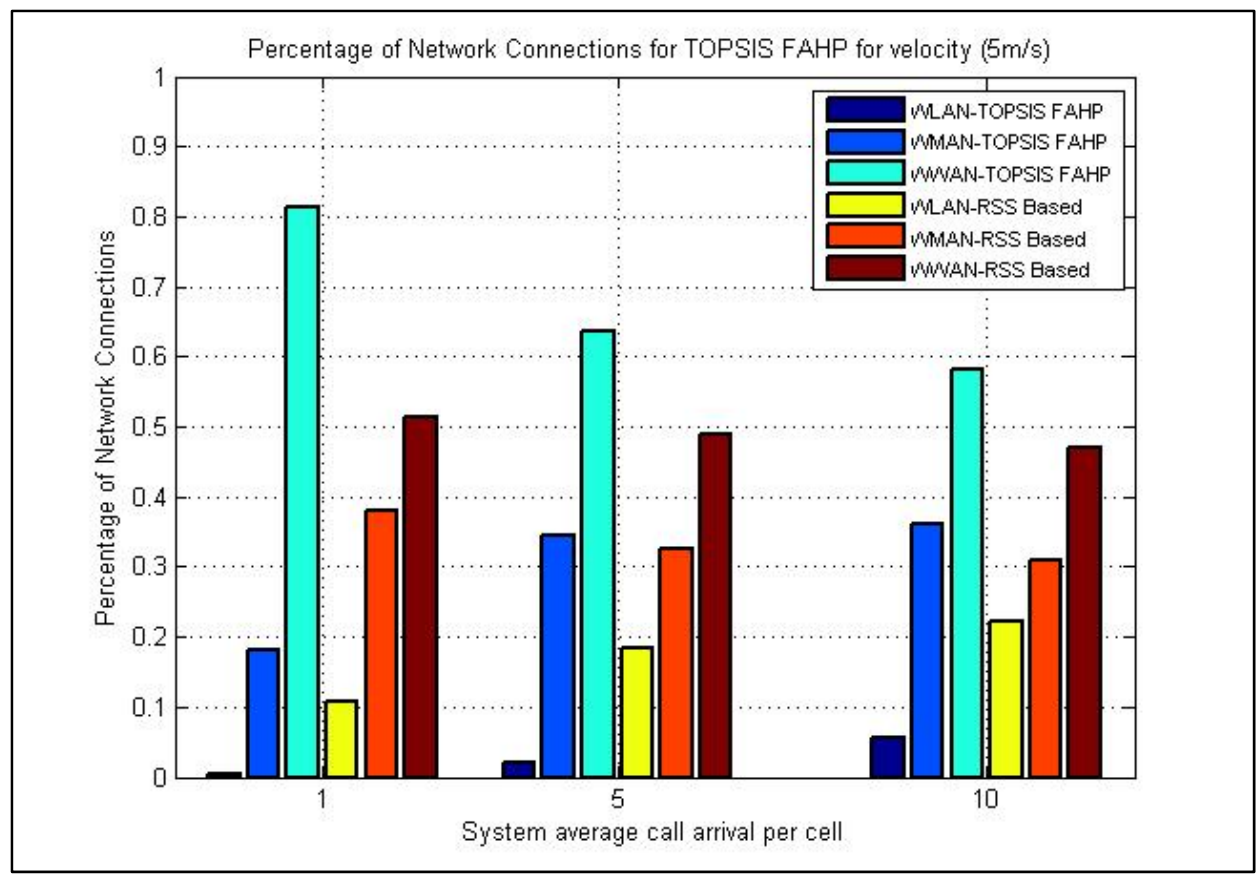

Figure 4.77: Percentage of NW-Connections per Number of Average Calls, TOPSIS-FAHP, Velocity $=5 \mathrm{~m} / \mathrm{s}$

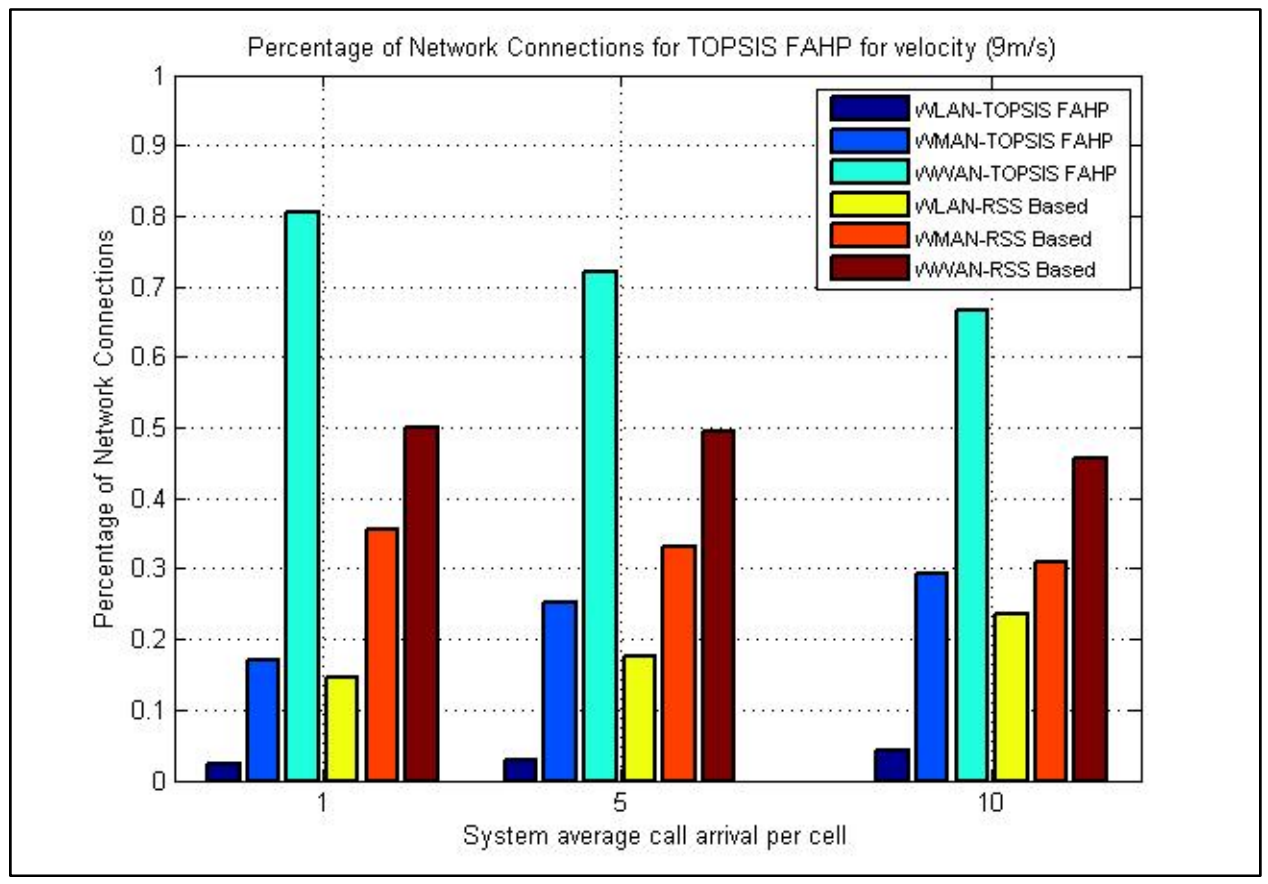

Figure 4.78: Percentage of NW-Connections per Number of Average Calls, TOPSIS-FAHP, Velocity $=9 \mathrm{~m} / \mathrm{s}$ 


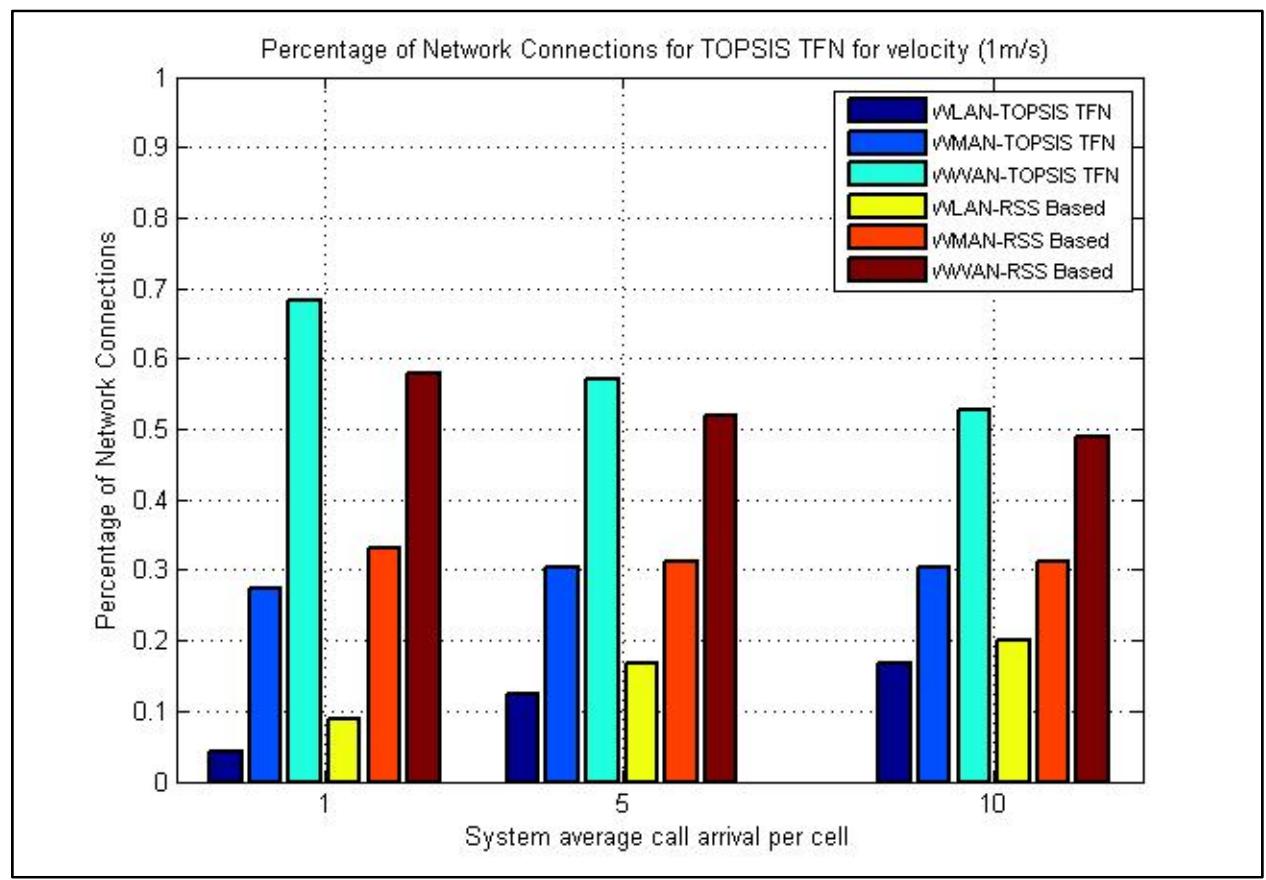

Figure 4.79: Percentage of NW-Connections per Number of Average Calls, TOPSIS-TFN, Velocity $=1 \mathrm{~m} / \mathrm{s}$

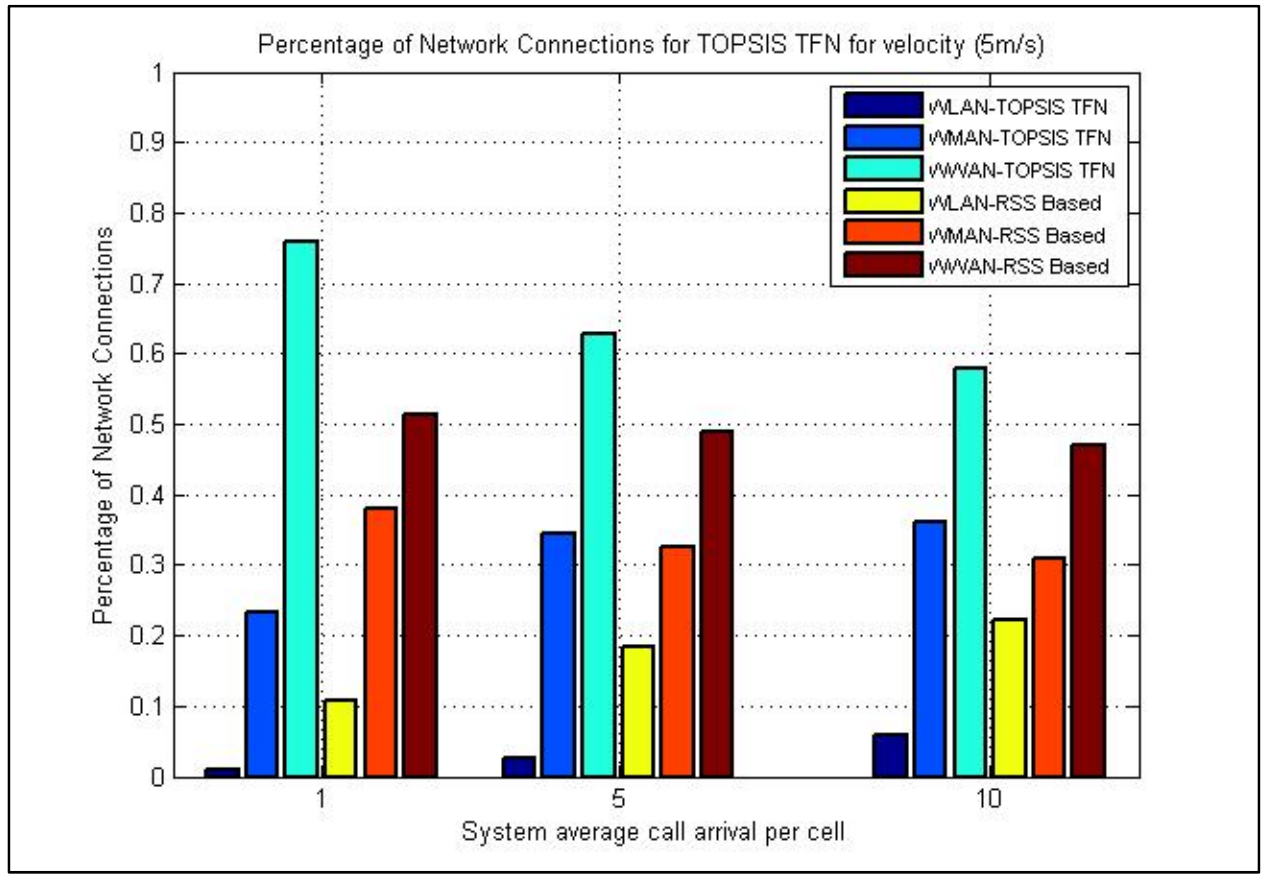

Figure 4.80: Percentage of NW-Connections per Number of Average Calls, TOPSIS-TFN, Velocity $=5 \mathrm{~m} / \mathrm{s}$ 


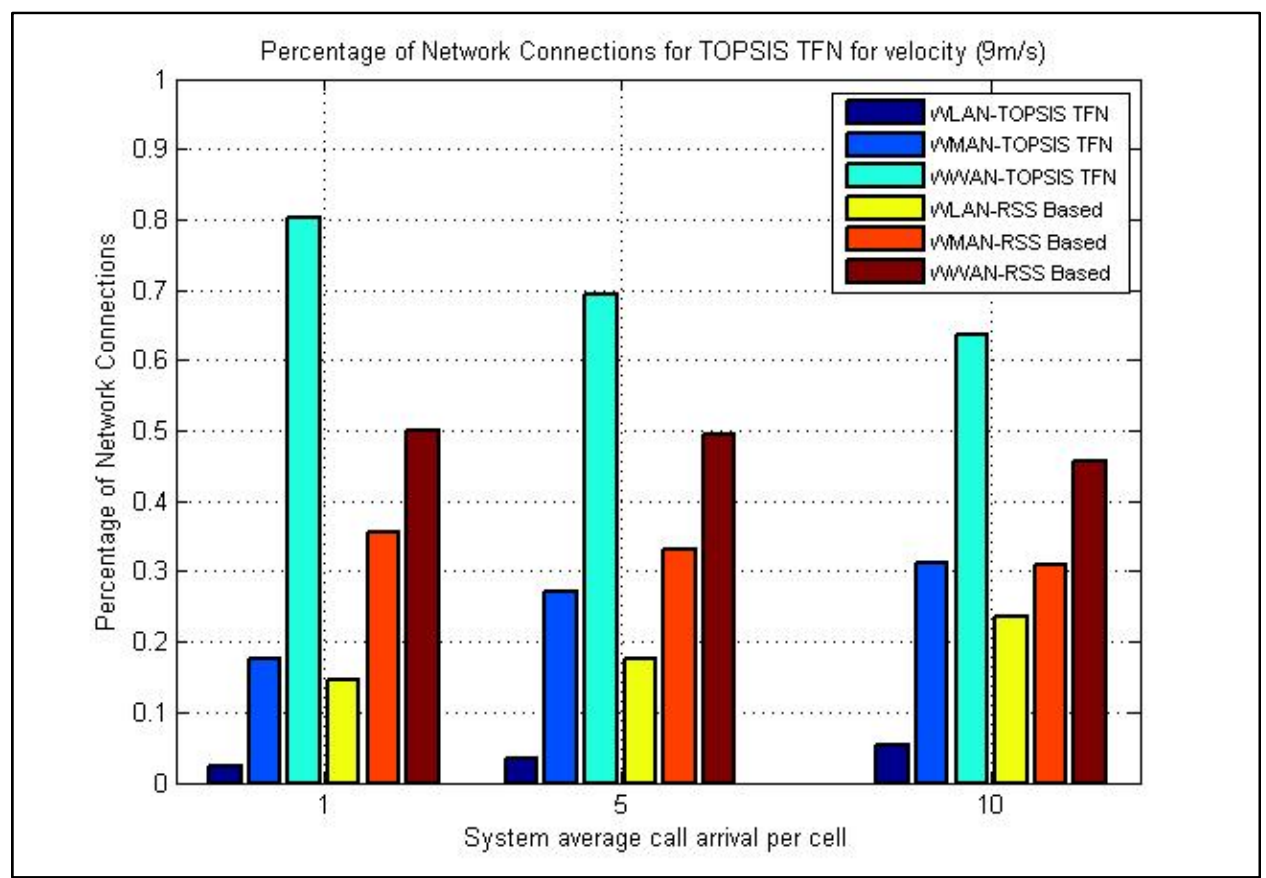

Figure 4.81: Percentage of NW-Connections per Number of Average Calls, TOPSIS-TFN, Velocity $=9 \mathrm{~m} / \mathrm{s}$

Based on the preceding discussions, it can be concluded that TOPSIS with FAHP or TFN perform better when compared with TOPSIS with AHP, which relies on crisp data.

\subsubsection{PERCENTAGE OF NETWORK CONNECTION BASED ON AVERAGE}

\section{SYSTEM CALLS-FTOPSIS AND FVIKOR}

The results for FTOPSIS and FVIKOR based network selection are presented in Figures 4.82-4.87. All the observations made for TOSPIS based schemes are also valid for FTOPSIS and FVIKOR target network selection schemes. Since both FTOPSIS and FVIKOR rely on fuzzy based network's attributes and weights, a higher preference can be seen towards WWAN. For example, approximately $87 \%$, and $58 \%$ preference towards WWAN can be observed from Figure 4.82, for an average system call rate of 1 and 10, 
respectively. For FTOPSIS, this preference is even higher for fast moving MSs; approximately $90 \%$ for system call arrival rate of 1 , as can be seen from Figure 4.84 .

For slow and medium speed MSs with a system call arrival rate of 10, FVIKOR performs nearly the same as the RSS based scheme. This is shown in Figure 4.85 and Figure 4.86 .

A comparison of FTOPSIS with FVIKOR reveals that VHITS selection scheme, based on FTOPSIS, produces better percentage of network connections at different MSsspeeds and at different system loading conditions. FTOPSIS also performs better than the TOPSIS based schemes as well as the traditional RSS based reference algorithm.

\subsection{COMPARISON BETWEEN DIFFERENT SCHEMES}

This chapter concludes by providing an overall comparison between the discussed schemes. This comparison is based on the same metrics discussed in Section 4.5 for evaluating VHITS in a multi-user scenario.

Tables 4.13-4.16 show comparisons among the different ranking algorithms that VHITS utilizes to perform vertical handoff decisions based on the four different traffic classes. All four tables depict different probabilities based on MSs' speed of 1, 5, and 10 $\mathrm{m} / \mathrm{s}$, assuming maximum system loading of 10 calls per cell. These tables also show the handoff rates based on the same values of MS-speed and system loading. The percentages of connections towards the three types of networks based on the four traffic classes can be observed as well from these tables. 


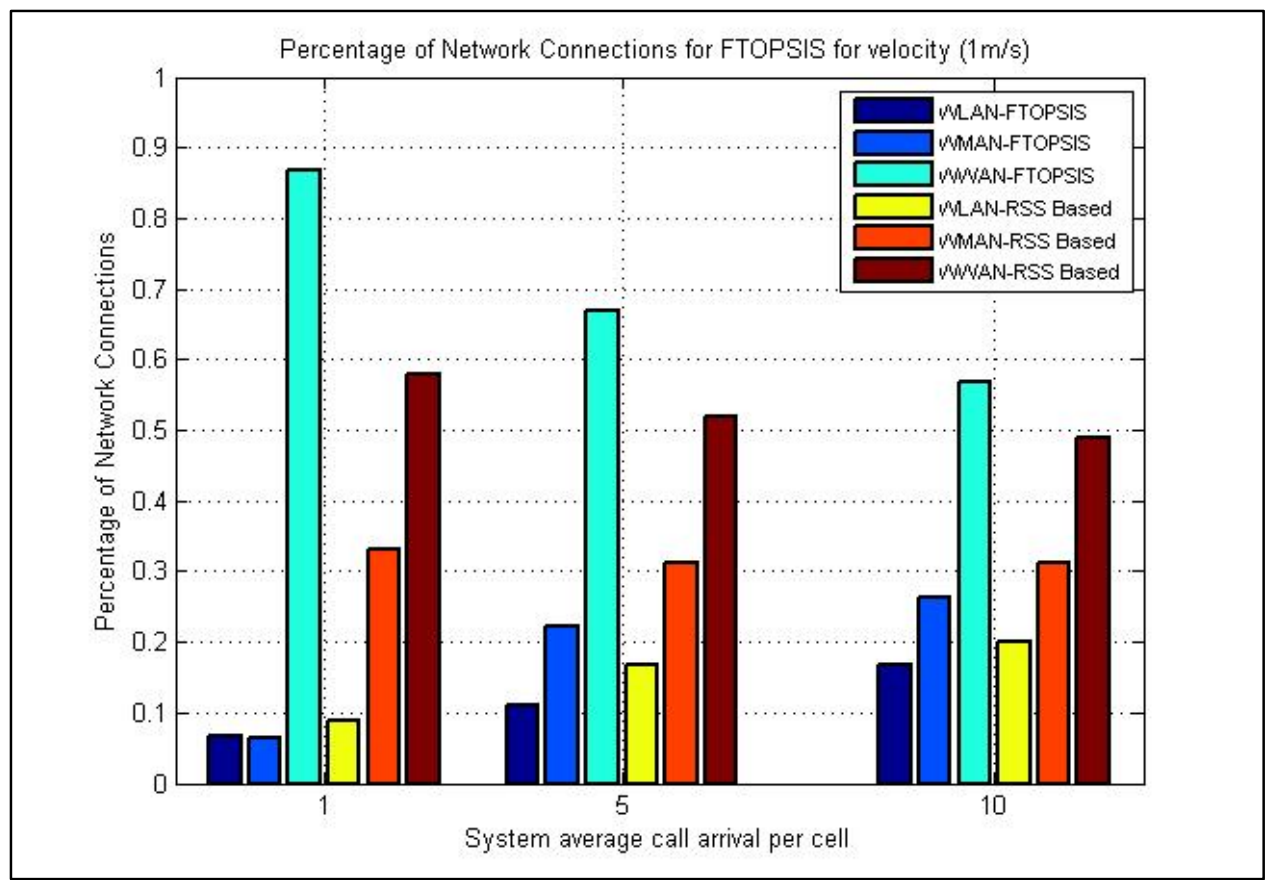

Figure 4.82: Percentage of NW-Connections per Number of Average Calls, FTOPSIS, Velocity $=1 \mathrm{~m} / \mathrm{s}$

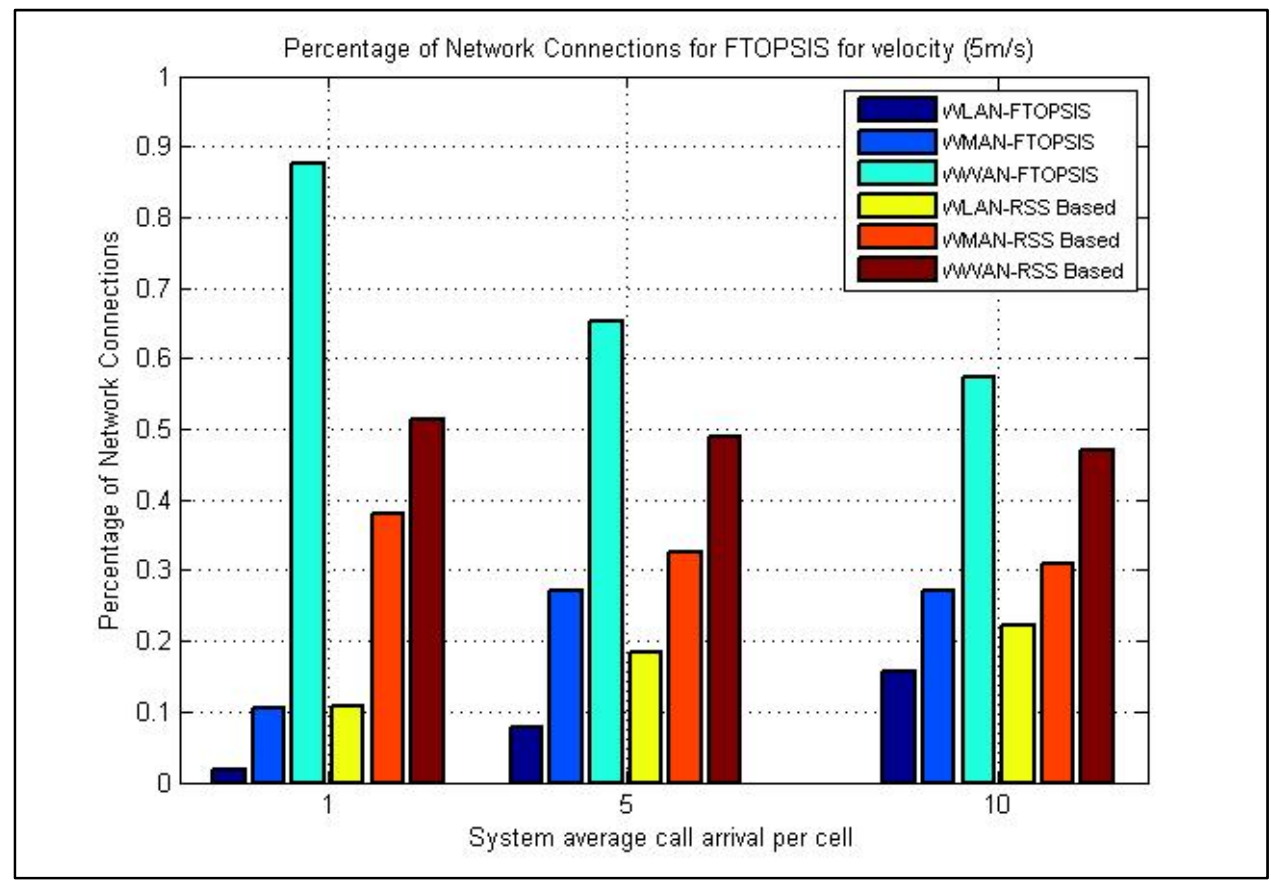

Figure 4.83: Percentage of NW-Connections per Number of Average Calls, FTOPSIS, Velocity $=5 \mathrm{~m} / \mathrm{s}$ 


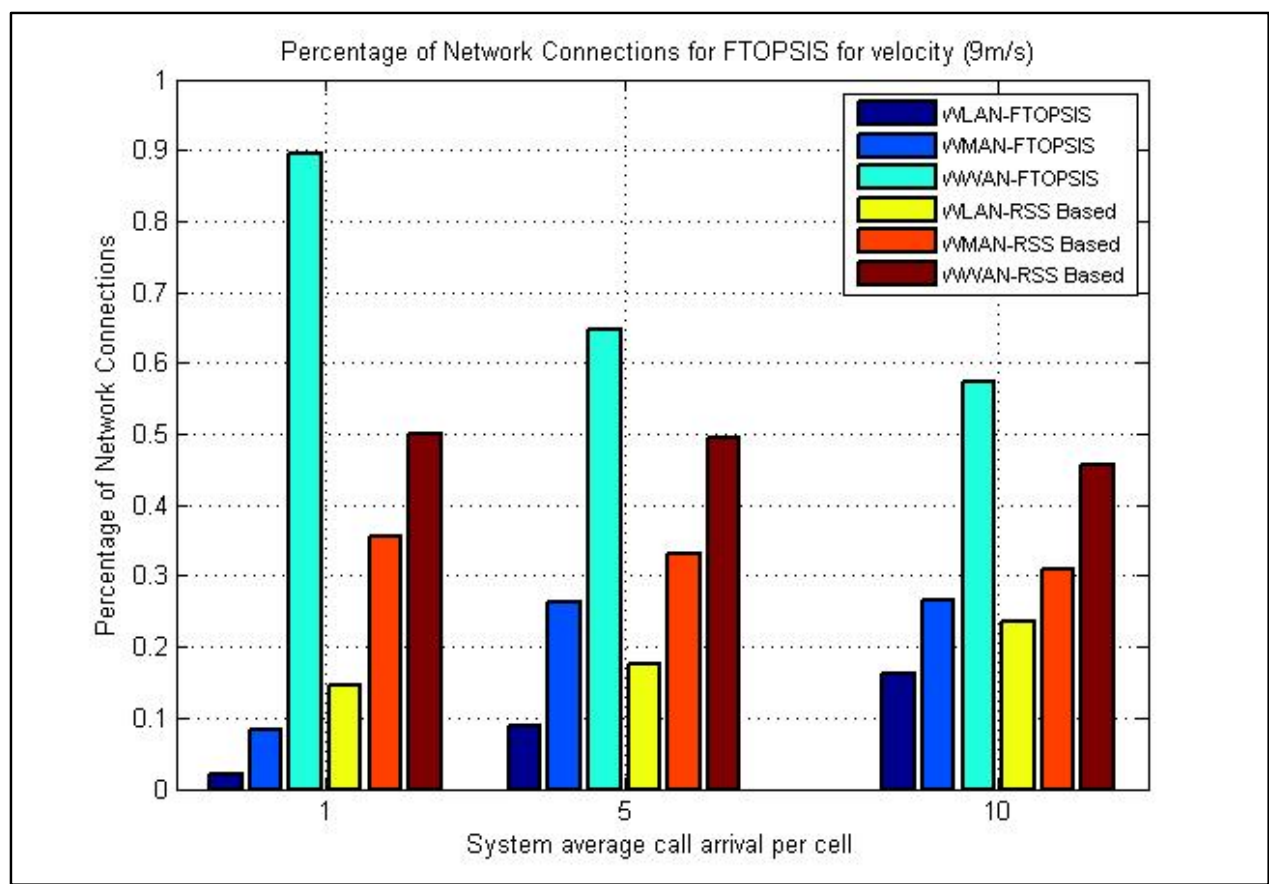

Figure 4.84: Percentage of NW-Connections per Number of Average Calls, FTOPSIS, Velocity $=9 \mathrm{~m} / \mathrm{s}$

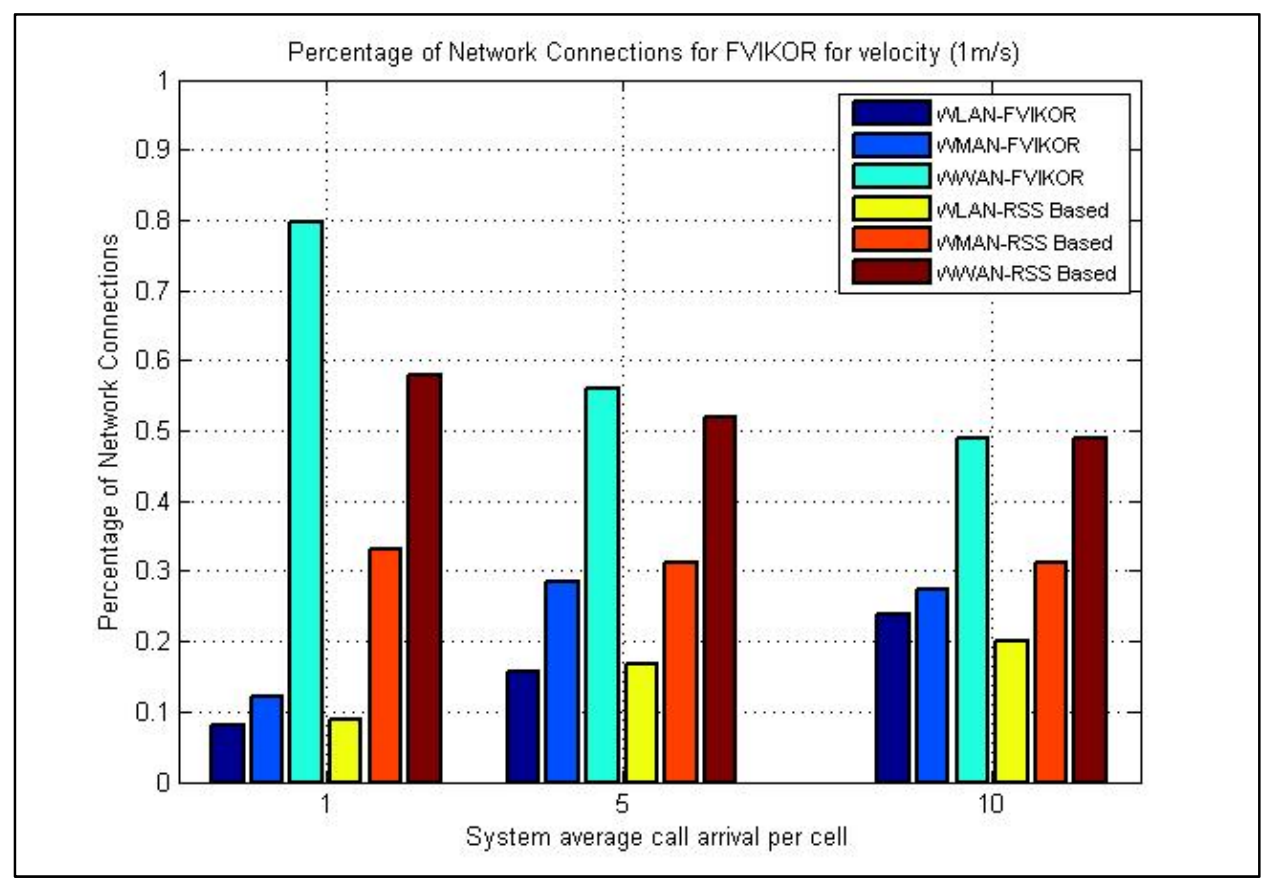

Figure 4.85: Percentage of NW-Connections per Number of Average Calls, FVIKOR, Velocity $=1 \mathrm{~m} / \mathrm{s}$ 


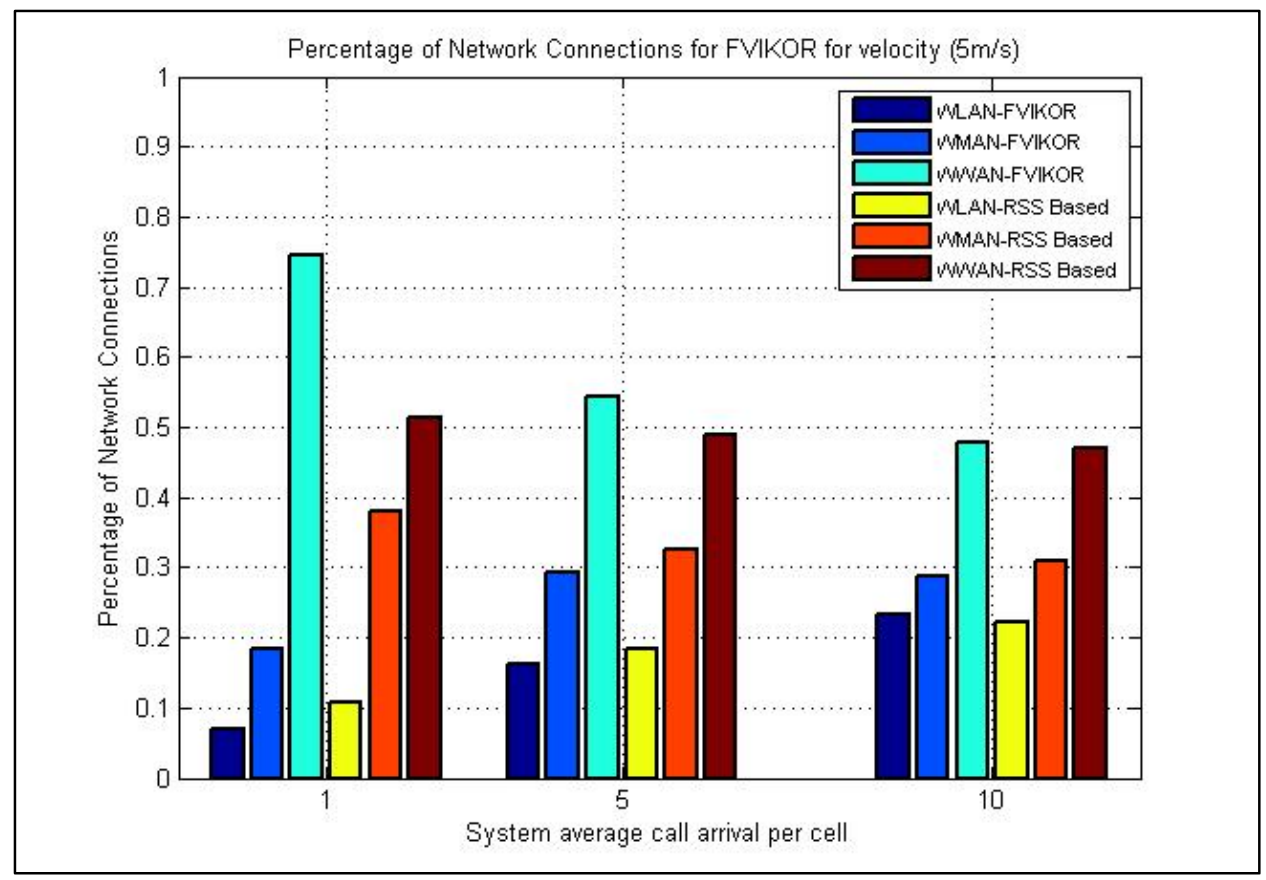

Figure 4.86: Percentage of NW-Connections per Number of Average Calls, FVIKOR, Velocity $=5 \mathrm{~m} / \mathrm{s}$

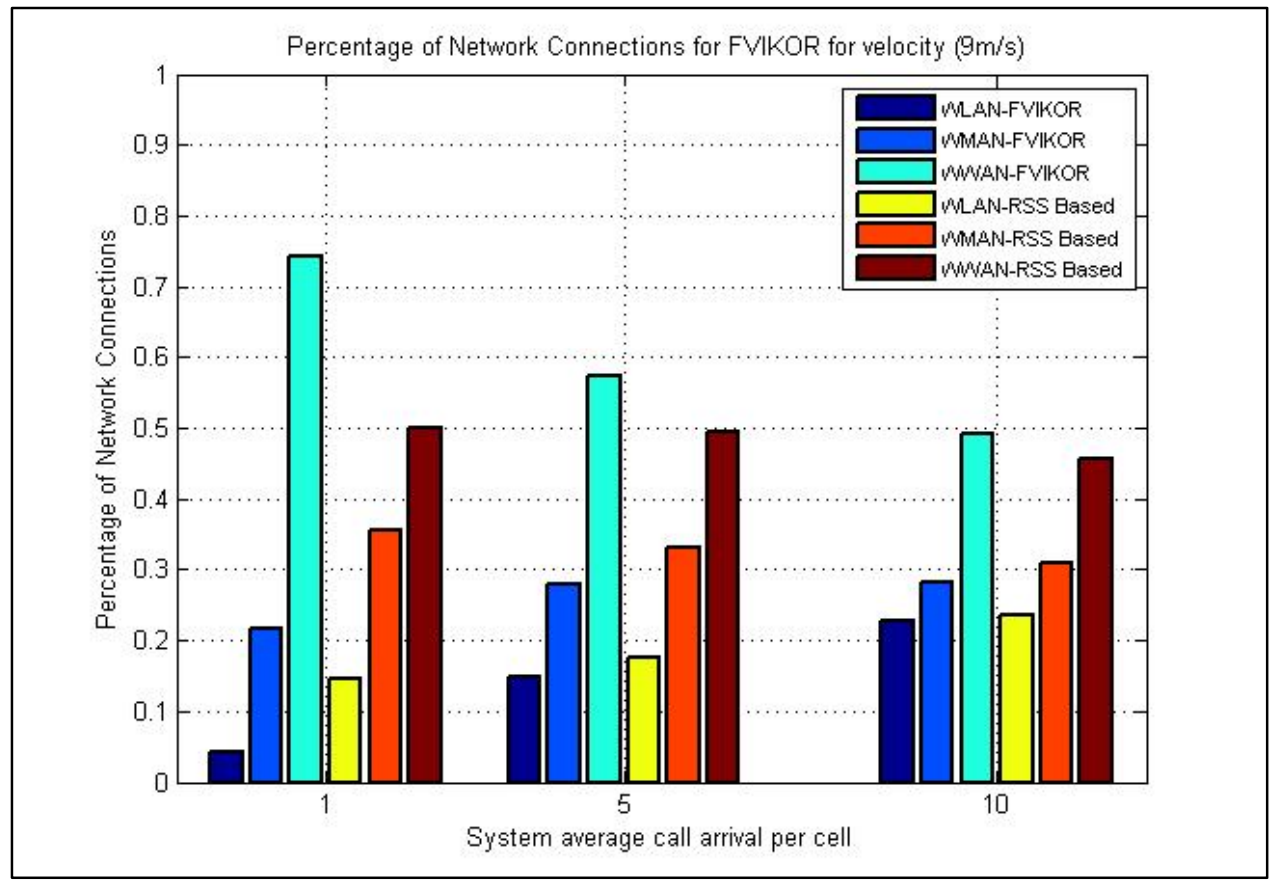

Figure 4.87: Percentage of NW-Connections per Number of Average Calls, FVIKOR, Velocity $=9 \mathrm{~m} / \mathrm{s}$ 
It can be seen that FTOPSIS outperforms all other schemes in terms of average outage probability, new call blocking probability, handoff blocking probability, average handoff rate, and percentage of connection towards a preferred network for all four traffic classes.

Table 4.13 shows the comparison between different selection schemes based on the Conversational traffic class. As can be seen from this table, at higher speeds and maximum system loadings, the VHITS scheme utilizing FTOPSIS performs $17 \%$ better than the reference algorithm in terms of average outage probability, $22 \%$ better in terms of new call blocking probability, and $16 \%$ better in terms of handoff blocking probability. The handoff rate also shows significant improvement of $40 \%$ over the reference algorithm, this being a clear indication of decreased "ping-pong effect" as well. The significant reduction in the handoff rate provided by the proposed scheme is translated into a better power consumption and more available battery life for MSs; as higher handoff rates consumes more battery power. As mentioned earlier, Conversational traffic class is characterized by low values of delay and jitter. Based on the current parameter set, these requirements are fulfilled by WWAN and followed by WMAN and WLAN. Higher connectivity preference towards WWAN can be observed from this table, for MSs utilizing the Conversational traffic class; the second preferred network is WMAN followed by WLAN, which offers the weakest QoS for Conversational traffic among all three networks.

The comparison of the schemes based on the Streaming traffic class is shown in Table 4.14. Just like Conversational traffic class, reduced values of different metrics can be seen for this class as well. As noted before, low value of jitter and a relatively higher 
value of throughput are required for Streaming traffic class. Based on the chosen parameters, WWAN provides the lowest value of jitter and WLAN provides the highest throughput. It can be observed from this table that WWAN provides the overall QoS required by the Streaming class. A connectivity preference can be seen towards WLAN for MSs moving at low speeds. A competition between WMAN and WLAN can also be observed for low MSs moving with low to mid speeds, this is due to WLAN providing better throughput when compared with WMAN.

Similar observations, in terms of lower outage and handoff rates, handoff blocking probabilities, and new call blocking probabilities, can also be made for the Background and Interactive traffic classes as can be seen from Table 4.15 and Table 4.16, respectively.

FVIKOR shows improvement in some areas especially when compared against the other non-fuzzy based and partially fuzzy based schemes. It can be noted that FVIKOR does not provide the same overall performance as FTOPSIS in terms of the metrics used to evaluate the proposed scheme.

The performance of TOPSIS-AHP for some metrics, such as new call and handoff blocking probability, is not good as compared with the RSS based reference algorithm. On the other hand, when subjected to fuzzy based weighting mechanisms such as FAHP or TFN, TOPSIS showed improved performance.

A comparison of FAHP weighting scheme with TFN reveals that TFN, which is based on simple usage of Linguistic Variables, mostly performs better than FAHP for all four traffic classes. 
We will conclude this chapter by making a final comment regarding the complexity of our scheme. Although, we have not performed a detailed complexity analysis in terms of resource usage, we believe that our scheme can be implemented on any smart mobile terminals since the latest innovations in mobile technology provides powerful processors and increased and efficient storage space; besides most of the latest consumer electronics such as digital cameras, are based on embedded fuzzy logic and provide superior performances. Just like any other complex MADM-based handoff scheme, we assume that the MS's battery consumption of our scheme is higher as well. Nonetheless, the proposed scheme, as noted from previous discussions, is able to achieve reduction in "ping-pong effect" by performing efficient and intelligent handoff decisions, which translate into better MS's power consumption. 
Table 4.13: VHITS Selection Schemes Comparison for Conversational Traffic Class

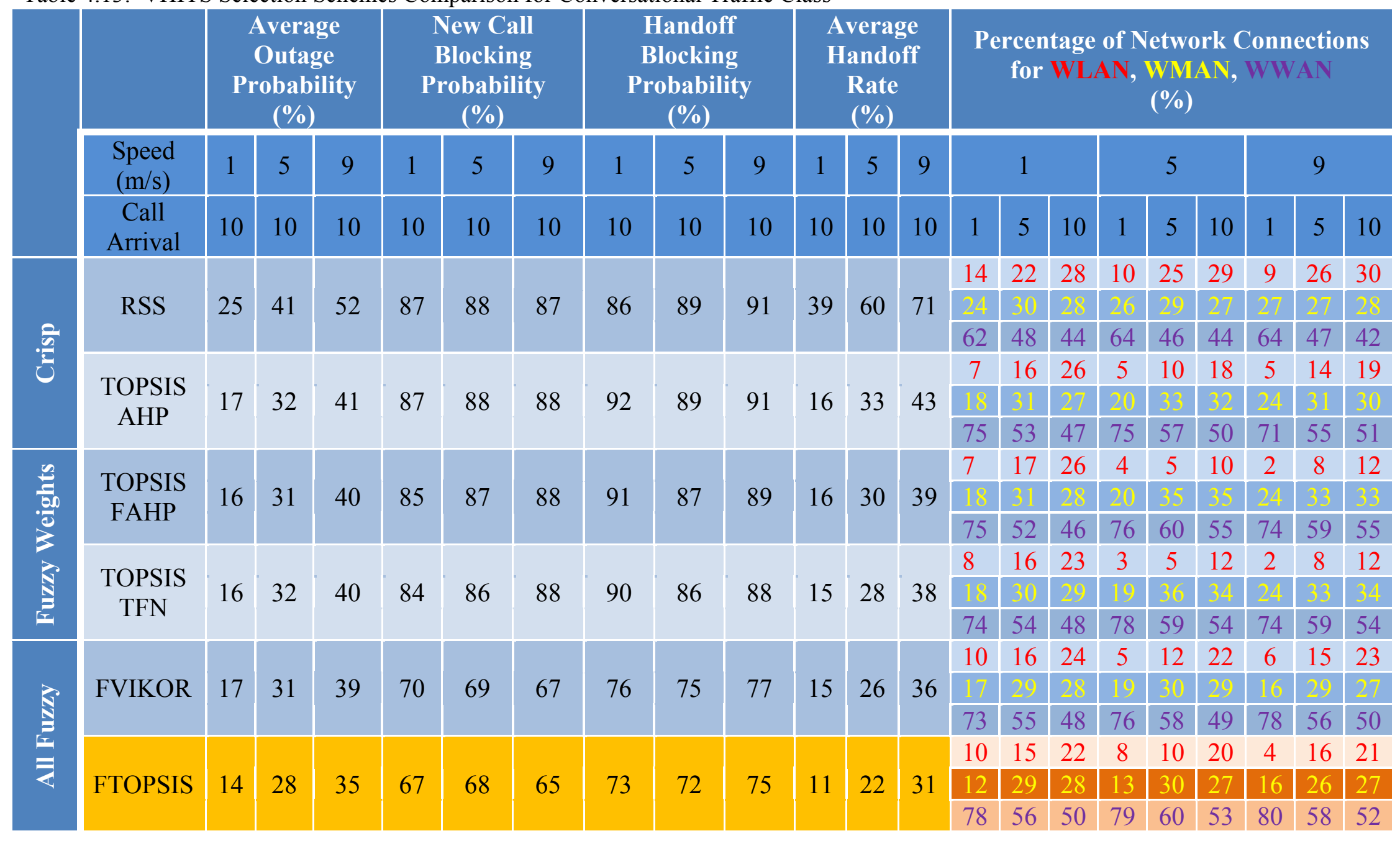


Table 4.14: VHITS Selection Schemes Comparison for Streaming Traffic Class

\begin{tabular}{|c|c|c|c|c|c|c|c|c|c|c|c|c|c|c|c|c|c|c|c|c|c|c|}
\hline & & \multicolumn{3}{|c|}{$\begin{array}{l}\text { Average } \\
\text { Outage } \\
\text { Probability } \\
(\%)\end{array}$} & \multicolumn{3}{|c|}{$\begin{array}{l}\text { New Call } \\
\text { Blocking } \\
\text { Probability } \\
(\%)\end{array}$} & \multicolumn{3}{|c|}{$\begin{array}{l}\text { Handoff } \\
\text { Blocking } \\
\text { Probability } \\
\text { (\%) }\end{array}$} & \multicolumn{3}{|c|}{$\begin{array}{l}\text { Average } \\
\text { Handoff } \\
\text { Rate } \\
(\%)\end{array}$} & \multicolumn{9}{|c|}{$\begin{array}{c}\text { Percentage of Network Connections } \\
\text { for WLAN, WMAN, WWAN } \\
(\%)\end{array}$} \\
\hline & \multirow{2}{*}{$\begin{array}{l}\begin{array}{l}\text { Speed } \\
(\mathrm{m} / \mathrm{s})\end{array} \\
\text { Call } \\
\text { Arrival }\end{array}$} & \multirow{2}{*}{10} & \multirow{2}{*}{5} & \multirow{2}{*}{9} & \multirow{2}{*}{10} & \multirow{2}{*}{5} & \multirow{2}{*}{9} & \multirow{2}{*}{1} & \multirow{2}{*}{5} & \multirow{2}{*}{9} & \multirow{2}{*}{10} & \multirow{2}{*}{$\frac{5}{10}$} & \multirow{2}{*}{$\begin{array}{c}9 \\
10\end{array}$} & \multicolumn{3}{|c|}{1} & \multicolumn{3}{|c|}{5} & \multicolumn{3}{|c|}{9} \\
\hline & & & & & & & & & & & & & & 1 & 5 & 10 & 1 & 5 & 10 & 1 & 5 & 10 \\
\hline \multirow{6}{*}{$\frac{0}{3}$} & \multirow{3}{*}{ RSS } & \multirow{3}{*}{25} & \multirow{3}{*}{41} & \multirow{3}{*}{52} & \multirow{3}{*}{87} & & & & & & & & & 14 & 22 & 28 & 10 & 25 & 29 & 9 & 26 & 30 \\
\hline & & & & & & 88 & 87 & 86 & 89 & 91 & 39 & 60 & 71 & 24 & 30 & 28 & 26 & 29 & 27 & 27 & 27 & 28 \\
\hline & & & & & & & & & & & & & & 62 & 48 & 44 & 64 & 46 & 44 & 64 & 47 & 42 \\
\hline & & & & & & & & & & & & & & 7 & 19 & 25 & 6 & 13 & 20 & 4 & 13 & 22 \\
\hline & $\begin{array}{c}\text { TUPSIS } \\
\text { A HP }\end{array}$ & 20 & 35 & 46 & 88 & 87 & 87 & 92 & 88 & 89 & 17 & 32 & 44 & 18 & 27 & 28 & 19 & 31 & 32 & 20 & 29 & 28 \\
\hline & & & & & & & & & & & & & & 75 & 54 & 47 & 75 & 56 & 48 & 76 & 58 & 50 \\
\hline & & & & & & & & & & & & & & 7 & 16 & 25 & 4 & 6 & 13 & 2 & 10 & 16 \\
\hline$\frac{5}{200}$ & $\begin{array}{l}\text { 1UPSIS } \\
\text { FAHP }\end{array}$ & 19 & 34 & 45 & 87 & 89 & 88 & 92 & 91 & 90 & 17 & 31 & 42 & 18 & 31 & 27 & 19 & 33 & 34 & 23 & 32 & 31 \\
\hline 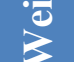 & & & & & & & & & & & & & & 75 & 53 & 48 & 77 & 61 & 53 & 75 & 58 & 53 \\
\hline 8 & & & & & & & & & & & & & & 8 & 15 & 23 & 4 & 7 & 14 & 2 & 11 & 15 \\
\hline $\mathbf{y}$ & TFN & 19 & 33 & 44 & 86 & 89 & 88 & 90 & 89 & 88 & 16 & 30 & 39 & 18 & 31 & 29 & 19 & 35 & 34 & 23 & 30 & 31 \\
\hline$E$ & & & & & & & & & & & & & & 74 & 54 & 48 & 77 & 58 & 52 & 75 & 59 & 54 \\
\hline & & & & & & & & & & & & & & 8 & 17 & 22 & 6 & 16 & 20 & 4 & 14 & 21 \\
\hline & FVIKOR & 18 & 32 & 43 & 72 & 71 & 71 & 77 & 76 & 78 & 15 & 28 & 40 & 16 & 29 & 29 & 22 & 30 & 30 & 20 & 30 & 30 \\
\hline 8 & & & & & & & & & & & & & & 76 & 54 & 49 & 72 & 54 & 50 & 76 & 56 & 49 \\
\hline$=$ & & & & & & & & & & & & & & 12 & 21 & 23 & 10 & 18 & 28 & 9 & 16 & 23 \\
\hline & FTOPSIS & 16 & 30 & 42 & 69 & 68 & 67 & 74 & 72 & 74 & 12 & 25 & 36 & 10 & 20 & 26 & 13 & 22 & 30 & 15 & 26 & 27 \\
\hline & & & & & & & & & & & & & & 78 & 59 & 51 & 77 & 60 & 52 & 76 & 58 & 50 \\
\hline
\end{tabular}


Table 4.15: VHITS Selection Schemes Comparison for Background Traffic Class

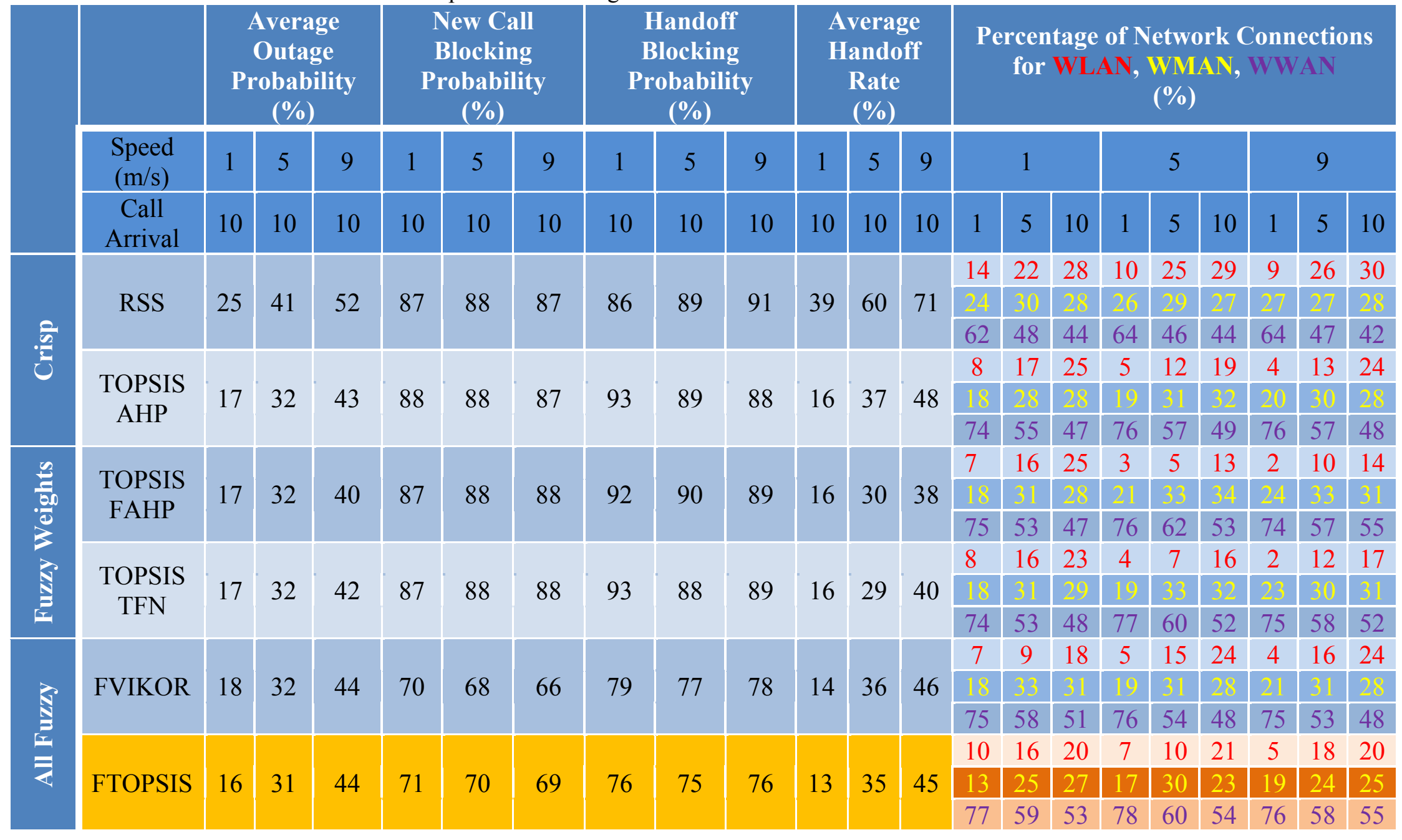


Table 4.16: VHITS Selection Schemes Comparison for Interactive Traffic Class

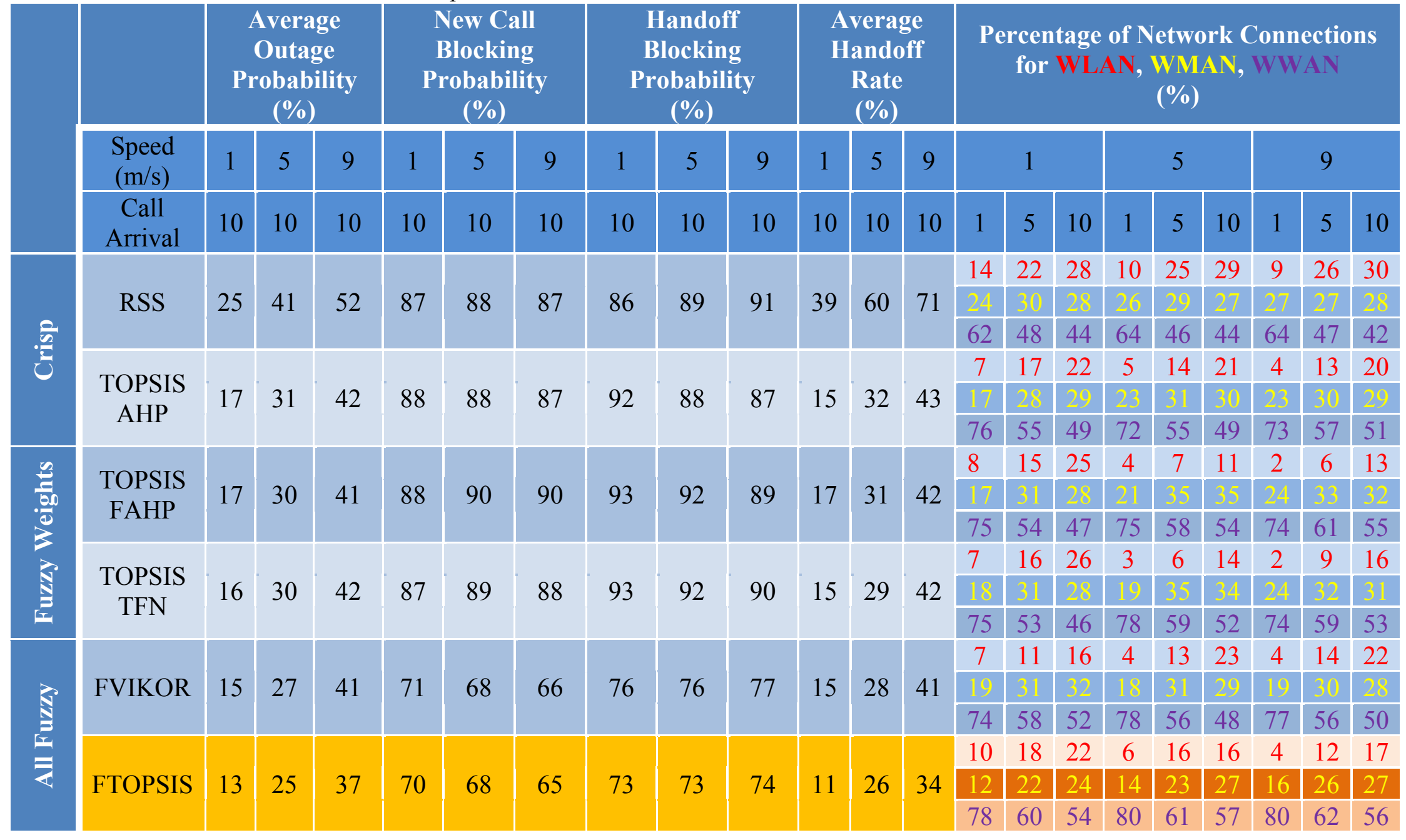




\section{CHAPTER 5}

\section{CONCLUSIONS AND FUTURE WORK}

This chapter summarizes the research work and discusses potential directions for future handoff-related research.

\section{$5.1 \quad$ SUMMARY}

In a highly integrated ubiquitous heterogeneous wireless environment, the selection of a network that can fulfill end-users' service requests while keeping their overall satisfaction at a very high level is vital; a wrong selection can lead to undesirable conditions such as unsatisfied users, weak QoS, network congestions, dropped and/or blocked calls, and wastage of valuable network resources. The selection of these networks is performed during the handoff process when an MS switches its current PoA to a different network due to the degradation or complete loss of signal and/or deterioration of the provided QoS. Traditional schemes perform the necessity of handoffs, and trigger the network selection process based on a single metric such as RSS. These schemes are not efficient and intelligent enough, so they do not take into consideration the traffic characteristics, user preferences, network conditions and other important system metrics.

The focus of this research work is on the design and implemention of a scheme that can perform efficient and intelligent vertical handoffs in heterogeneous wireless networks. The main objective of the developed scheme is to minimize the number of unnecessary handoffs while maximizing the sojourn time with a preferred network, resulting in increased end-users' satisfaction levels. 
Two modules are proposed, namely VHITS Handoff Necessity Estimation, and VHITS Handoff Target Network Selection. The fuzzy logic-based VHITS Handoff Necessity Estimation module determines whether a handoff is necessary by taking into consideration the predicted RSS values provided by the current PoA, the degree of the provided QoS based on the requested traffic class (Conversational, Streaming, Background, and Interactive), and the speed of the vehicle including the direction (toward/away from the PoA) in which the MS is travelling. The future value of RSS predicted using GPT is used to minimize call dropping probabilities due to sudden loss of signal in a lognormal fading environment that is inherent in wireless networks. Several parallel FLCs are designed to make the computation of the proposed scheme efficient by minimizing the number of required inference rules.

The VHITS Target Selection scheme also utilizes fuzzy logic in addition to different ranking algorithms and weight elicitation techniques that are implemented to select the best target network that can fulfill end-users' preferences. Multiple weighting schemes are developed: AHP uses crisp values to indicate user preferences while FAHP, TFN, and Linguistic Variables based schemes are developed to deal with uncertainty and vagueness in user-provided preferences by treating them as fuzzy data. Multiple ranking algorithms are implemented and compared to perform network selection: TOPSIS uses crisp values for multiple networks' attributes required to make the selection, while FTOPSIS and FVIKOR are based on fuzzy data. These schemes are implemented to deal with uncertain and fuzzy values of the measured parameters inherent in a dynamic wireless environment. The carefully chosen network attributes from WLAN, WMAN, and WWAN include predicted RSS, QoS-related parameters (delay, jitter, PLR, and 
throughput), and speed of the MS including its moving direction, traffic-loading conditions, security preferences, and the cost of the provided service. Four different types of services, namely Conversational, Streaming, Background, and Interactive, are considered in this research work.

As noted from the discussion above, the developed modules utilize the benefits of parallel FLCs, and different weighting and MADM algorithms that can work with both crisp and fuzzy data. Furthermore, the proposed scheme is flexible and scalable as it can work with any number of networks with a large set of decision criteria. These are obvious advantages of using this scheme over traditional ones as well as other schemes that utilize multiple metrics, to perform handoff decisions.

Numerical examples are provided and simulations using RUNE tools are done comparing the performance of the proposed scheme with the traditional reference algorithms. Simulations based on certain types of traffic classes are also carried out comparing the performance of implemented weighing schemes as well as the network ranking algorithms. The simulation results indicate a better overall performance of the proposed scheme in terms of minimized handoffs, lower probability of handoff blocking and new call blocking, and increased connection time with the selected network that is preferred by the end-user. The VHITS necessity estimation scheme is able to reduce the number of handoffs, and the VHITS target network selection scheme based on FTOPSIS outperforms the other schemes implemented and simulated in terms of intelligent and efficient handoff decisions. 


\subsection{FUTURE WORK}

Different aspects of the proposed scheme can be further improved. Some of the major future directions related to handoff research are briefly discussed as follows:

- The handoff process is just one aspect of an overall mobility framework to provide ubiquitous access to MSs moving in a heterogeneous wireless environment. The proposed handoff algorithm can be combined with other resource management tasks such as power and call admission control, and channel assignments to provide continued and guaranteed quality of service. A joint optimization of such an integrated system may result in an overall optimal performance with increased users' satisfaction.

- We intend to optimize the proposed weight elicitation process to improve the efficiency of handoffs for each of the four traffic classes. This optimization can be achieved through the use of neural or Bayesian networks.

- The proposed scheme is based on fuzzy logic with rules and membership functions that are subjective in nature. Hence, research needs to be done in finding out the different types of membership functions that can result in optimal handoff performance. One possibility is to utilize an AdaptiveNetwork-based Fuzzy Inference System (ANFIS) where the system can construct an input-output relationship based on both subjective human knowledge and stipulated input-output data pairs. By embedding the fuzzy inference system into the framework of adaptive networks, a set of fuzzy if-then rules with appropriate membership functions is created to generate 
the stipulated input-output pairs. Another possibility includes suitable learning methods such as neural and Bayesian networks.

- The proposed scheme is tested using RUNE that can provide propagation data based on different mobility and path models. Further improvements may be achieved by optimizing the algorithm using real propagation data and by testing its performance utilizing real wireless network conditions.

- The proposed scheme investigates handoffs using three types of networks, namely WLAN, WMAN, and WWAN, without targeting a specific wireless network technology. We plan to extend this research work by applying this newly developed scheme to the handoff process of specific wireless technologies such as LTE advance, $60 \mathrm{GHz}$ millimeter waves, Bluetooth, IEEE 802.11, and IEEE 802.16.

- We plan to perform a detailed complexity analysis in terms of resources usage to determine the feasibility of our proposed scheme. As MS-battery consumption is critical in any MADM based scheme, we intend to extend our work by offering a power-efficient handoff scheme that can provide similar intelligent handoff decisions. 


\section{REFERENCES}

[1] Xiaohuan Yan, N. Mani and Y. A. Cekercioglu, "A Traveling Distance Prediction Based Method to Minimize Unnecessary Handovers from Cellular Networks to WLANs," Communications Letters, IEEE, vol. 12, pp. 14-16, 2008.

[2] I. F. Akyildiz, Jiang Xie and S. Mohanty, "A survey of mobility management in nextgeneration all-IP-based wireless systems," Wireless Communications, IEEE, vol. 11, pp. 16-28, 2004.

[3] C. Çeken, S. Yarkan and H. Arslan, "Interference aware vertical handoff decision algorithm for quality of service support in wireless heterogeneous networks," Computer Networks, vol. 54, pp. 726-740, 4/8, 2010.

[4] S. Dekleva, J. P. Shim, U. Varshney and G. Knoerzer, "Evolution and emerging issues in mobile wireless networks," Commun ACM, vol. 50, pp. 38-43, June, 2007.

[5] Q. -. Nguyen-Vuong, N. Agoulmine and Y. Ghamri-Doudane, "Terminal-Controlled Mobility Management in Heterogeneous Wireless Networks," Communications Magazine, IEEE, vol. 45, pp. 122-129, 2007.

[6] Xichun Li, A. Gani, R. Salleh and O. Zakaria, "The future of mobile wireless communication networks," in Communication Software and Networks, 2009. ICCSN '09. International Conference on, 2009, pp. 554-557.

[7] M. Bernaschi, F. Cacace, G. lannello and M. Vellucci, "Mobility Management for VoIP on Heterogeneous Networks: Evaluation of Adaptive Schemes," Mobile Computing, IEEE Transactions on, vol. 6, pp. 1035-1047, 2007.

[8] L. Eastwood, S. Migaldi, Qiaobing Xie and V. Gupta, "Mobility using IEEE 802.21 in a heterogeneous IEEE 802.16/802.11-based, IMT-advanced (4G) network," Wireless Communications, IEEE, vol. 15, pp. 26-34, 2008.

[9] K. V. S. S. S. S. Sairam, N. Gunasekaran and S. R. Redd, "Bluetooth in wireless communication," Communications Magazine, IEEE, vol. 40, pp. 90-96, 2002.

[10] Der-Jiunn Deng and Hsu-Chun Yen, "Quality-of-service provisioning system for multimedia transmission in IEEE 802.11 wireless LANs," Selected Areas in Communications, IEEE Journal on, vol. 23, pp. 1240-1252, 2005.

[11] Fan Wang, A. Ghosh, C. Sankaran, P. Fleming, F. Hsieh and S. Benes, "Mobile WiMAX systems: performance and evolution," Communications Magazine, IEEE, vol. 46, pp. 41-49, 2008. 
[12] G. Lampropoulos, A. K. Salkintzis and N. Passas, "Media-independent handover for seamless service provision in heterogeneous networks," Communications Magazine, IEEE, vol. 46, pp. 64-71, 2008.

[13] I. F. Akyildiz, J. McNair, J. S. M. Ho, H. Uzunalioglu and Wenye Wang, "Mobility management in next-generation wireless systems," Proceedings of the IEEE, vol. 87, pp. 1347-1384, 1999.

[14] M. Kassar, B. Kervella and G. Pujolle, "An overview of vertical handover decision strategies in heterogeneous wireless networks," Comput. Commun., vol. 31, pp. 26072620, 6/25, 2008.

[15] F. Zhu and J. McNair, "Multiservice vertical handoff decision algorithms," EURASIP J.Wirel.Commun.Netw., vol. 2006, pp. 52-52, April, 2006.

[16] J. Kang, J. Strassner, S. Seo and J. W. Hong, "Autonomic personalized handover decisions for mobile services in heterogeneous wireless networks," Computer Networks, vol. 55, pp. 1520-1532, 5/16, 2011.

[17] F. Siddiqui and S. Zeadally, "Mobility management across hybrid wireless networks: Trends and challenges," Comput. Commun., vol. 29, pp. 1363-1385, May, 2006.

[18] N. Nasser, A. Hasswa and H. Hassanein, "Handoffs in fourth generation heterogeneous networks," Communications Magazine, IEEE, vol. 44, pp. 96-103, 2006.

[19] N. D. Tripathi, J. H. Reed and H. F. VanLandinoham, "Handoff in cellular systems," Personal Communications, IEEE, vol. 5, pp. 26-37, 1998.

[20] I. F. Akyildiz and W. Wang, "A dynamic location management scheme for nextgeneration multitier PCS systems," Wireless Communications, IEEE Transactions on, vol. 1, pp. 178-189, 2002.

[21] E. Stevens-Navarro and V. W. S. Wong, "Comparison between vertical handoff decision algorithms for heterogeneous wireless networks," in Vehicular Technology Conference, 2006. VTC 2006-Spring. IEEE 63rd, 2006, pp. 947-951.

[22] A. H. Zahran and Ben Liang, "Performance evaluation framework for vertical handoff algorithms in heterogeneous networks," in Communications, 2005. ICC 2005. 2005 IEEE International Conference on, 2005, pp. 173-178 Vol. 1.

[23] W. C. Y. Lee, Mobile Cellular Telecommunications Systems. New York: McGrawHill, 1989. 
[24] Ning Zhang and J. M. Holtzman, "Analysis of handoff algorithms using both absolute and relative measurements," in Vehicular Technology Conference, 1994 IEEE 44th, 1994, pp. 82-86 vol.1.

[25] A. H. Zahran, B. Liang and A. Saleh, "Signal threshold adaptation for vertical handoff in heterogeneous wireless networks," Mob.Netw.Appl., vol. 11, pp. 625-640, August, 2006.

[26] M. D. Austin and G. L. Stuber, "Direction biased handoff algorithms for urban microcells," in Vehicular Technology Conference, 1994 IEEE 44th, 1994, pp. 101-105 vol.1.

[27] R. Tawil, G. Pujolle and O. Salazar, "A vertical handoff decision scheme in heterogeneous wireless systems," in Vehicular Technology Conference, 2008. VTC Spring 2008. IEEE, 2008, pp. 2626-2630.

[28] H. Maturino-Lozoya, D. Munoz-Rodriguez and H. Tawfik, "Pattern recognition techniques in handoff and service area determination," in Vehicular Technology Conference, 1994 IEEE 44th, 1994, pp. 96-100 vol.1.

[29] A. Mehbodniya and J. Chitizadeh, "An intelligent vertical handoff algorithm for next generation wireless networks," in Wireless and Optical Communications Networks, 2005. WOCN 2005. Second IFIP International Conference on, 2005, pp. 244-249.

[30] V. Kapor, G. Edwards and R. Sankar, "Handoff criteria for personal communication networks," in Communications, 1994. ICC '94, SUPERCOMM/ICC '94, Conference Record, 'Serving Humanity through Communications.' IEEE International Conference on, 1994, pp. 1297-1301 vol.3.

[31] Liu Xia, Ling-ge Jiang and Chen He, "A novel fuzzy logic vertical handoff algorithm with aid of differential prediction and pre-decision method," in Communications, 2007. ICC '07. IEEE International Conference on, 2007, pp. 56655670 .

[32] N. Nasser, S. Guizani and E. Al-Masri, "Middleware vertical handoff manager: A neural network-based solution," in Communications, 2007. ICC '07. IEEE International Conference on, 2007, pp. 5671-5676.

[33] Kwong Chiew Foong, Chuah Teong Chee and Lee Sze Wei, "Adaptive network fuzzy inference system (ANFIS) handoff algorithm," in Future Computer and Communication, 2009. ICFCC 2009. International Conference on, 2009, pp. 195-198.

[34] K. Taniuchi, Y. Ohba, V. Fajardo, S. Das, M. Tauil, Yuu-Heng Cheng, A. Dutta, D. Baker, M. Yajnik and D. Famolari, "IEEE 802.21: Media independent handover: 
Features, applicability, and realization," Communications Magazine, IEEE, vol. 47, pp. 112-120, 2009.

[35] S. Mohanty and I. F. Akyildiz, "A Cross-Layer (Layer 2 + 3) Handoff Management Protocol for Next-Generation Wireless Systems," Mobile Computing, IEEE Transactions on, vol. 5, pp. 1347-1360, 2006.

[36] Young-Il Kim, Kyung-Jun Lee and Yong-Oak Chin, "Analysis of multi-level threshold handoff algorithm," in Global Telecommunications Conference, 1996. GLOBECOM '96. 'Communications: The Key to Global Prosperity, 1996, pp. 1141-1145 vol.2.

[37] Li Hua, M. H. Kabir and T. Sato, "Velocity adaptive vertical handoff on multifrequency system," in Personal, Indoor and Mobile Radio Communications, 2009 IEEE 20th International Symposium on, 2009, pp. 773-777.

[38] M. D. Austin and G. L. Stuber, "Velocity adaptive handoff algorithms for microcellular systems," Vehicular Technology, IEEE Transactions on, vol. 43, pp. 549$561,1994$.

[39] Li Zheng, Wang Yu and Wu Weiling, "Position location and direction assisted handoff algorithm in DWCS," in Personal, Indoor and Mobile Radio Communications, 2004. PIMRC 2004. 15th IEEE International Symposium on, 2004, pp. 663-666 Vol.1.

[40] N. D. Tripathi, J. H. Reed and H. F. VanLandingham, "An adaptive direction biased fuzzy handoff algorithm with unified handoff candidate selection criterion," in Vehicular Technology Conference, 1998. VTC 98. 48th IEEE, 1998, pp. 127-131 vol.1.

[41] Chen-Nee Chuah and R. D. Yates, "Evaluation of a minimum power handoff algorithm," in Personal, Indoor and Mobile Radio Communications, 1995. PIMRC'95. 'Wireless: Merging Onto the Information Superhighway'., Sixth IEEE International Symposium on, 1995, pp. 814-818 vol.2.

[42] A. Calvagna and G. Di Modica, "A user-centric analysis of vertical handovers," in Proceedings of the 2nd ACM International Workshop on Wireless Mobile Applications and Services on WLAN Hotspots, Philadelphia, PA, USA, 2004, pp. 137-146.

[43] A. Hasswa, N. Nasser and H. Hossanein, "Generic vertical handoff decision function for heterogeneous wireless," in Wireless and Optical Communications Networks, 2005. WOCN 2005. Second IFIP International Conference on, 2005, pp. 239-243.

[44] O. Ormond, G. Miro-Muntean and J. Murphy, "Evaluation of an intelligent utilitybased strategy for dynamic wireless network selection," in Proceedings of the 9th IFIP/IEEE International Conference on Management of Multimedia and Mobile Networks and Services, Dublin, Ireland, 2006, pp. 158-170. 
[45] Q. Nguyen-Vuong, N. Agoulmine and Y. Ghamri-Doudane, "A user-centric and context-aware solution to interface management and access network selection in heterogeneous wireless environments," Comput.Netw., vol. 52, pp. 3358-3372, December, 2008.

[46] Q. Wei, K. Farkas, C. Prehofer, P. Mendes and B. Plattner, "Context-aware handover using active network technology," Comput.Netw., vol. 50, pp. 2855-2872, October, 2006.

[47] T. Ahmed, K. Kyamakya and M. Ludwig, "Design and implementation of a contextaware decision algorithm for heterogeneous networks," in Proceedings of the 2006 ACM Symposium on Applied Computing, Dijon, France, 2006, pp. 1134-1138.

[48] B. S. Ghahfarokhi and N. Movahhedinia, "A context-aware handover decision based on user perceived quality of service trigger," Wirel.Commun.Mob.Comput., vol. 11, pp. 723-741, June, 2011.

[49] Miao Xiong, Jiannong Cao and Jun Zhang, "Context-aware mechanism for IEEE 802.21 media independent handover," in Computer Communications and Networks (ICCCN), 2011 Proceedings of 20th International Conference on, 2011, pp. 1-6.

[50] P. TalebiFard and V. C. M. Leung, "A dynamic context-aware access network selection for handover in heterogeneous network environments," in Computer Communications Workshops (INFOCOM WKSHPS), 2011 IEEE Conference on, 2011, pp. 385-390.

[51] M. Zekri, B. Jouaber and D. Zeghlache, "Context aware vertical handover decision making in heterogeneous wireless networks," in Local Computer Networks (LCN), 2010 IEEE 35th Conference on, 2010, pp. 764-768.

[52] T. Ahmed, K. Kyamakya and M. Ludwig, "Architecture of a context-aware vertical handover decision model and its performance analysis for GPRS - WiFi handover," in Computers and Communications, 2006. ISCC '06. Proceedings. 11th IEEE Symposium on, 2006, pp. 795-801.

[53] J. McNair and Fang Zhu, "Vertical handoffs in fourth-generation multinetwork environments," Wireless Communications, IEEE, vol. 11, pp. 8-15, 2004.

[54] H. J. Wang, R. H. Katz and J. Giese, "Policy-enabled handoffs across heterogeneous wireless networks," in Mobile Computing Systems and Applications, 1999. Proceedings. WMCSA '99. Second IEEE Workshop on, 1999, pp. 51-60.

[55] Wen-Tsuen Chen, Jen-Chu Liu and Hsieh-Kuan Huang, "An adaptive scheme for vertical handoff in wireless overlay networks," in Parallel and Distributed Systems, 
2004. ICPADS 2004. Proceedings. Tenth International Conference on, 2004, pp. 541548.

[56] M. Angermann and J. Kammann, "Cost metrics for decision problems in wireless ad hoc networking," in In Proceedings IEEE CAS 2002, 2002, .

[57] Fang Zhu and J. McNair, "Optimizations for vertical handoff decision algorithms," in Wireless Communications and Networking Conference, 2004. WCNC. 2004 IEEE, 2004, pp. 867-872 Vol.2.

[58] E. Stevens-Navarro, Yuxia Lin and V. W. S. Wong, "An MDP-Based Vertical Handoff Decision Algorithm for Heterogeneous Wireless Networks," Vehicular Technology, IEEE Transactions on, vol. 57, pp. 1243-1254, 2008.

[59] Su Kyoung Lee, K. Sriram, Kyungsoo Kim, Jong Hyup Lee, Yoon Hyuk Kim and N. Golmie, "Vertical handoff decision algorithm providing optimized performance in heterogeneous wireless networks," in Global Telecommunications Conference, 2007. GLOBECOM '07. IEEE, 2007, pp. 5164-5169.

[60] F. Bari and V. Leung, "Application of ELECTRE to network selection in A hetereogeneous wireless network environment," in Wireless Communications and Networking Conference, 2007.WCNC 2007. IEEE, 2007, pp. 3810-3815.

[61] J. D. Martínez-Morales, U. Pineda-Rico and E. Stevens-Navarro, "Performance comparison between MADM algorithms for vertical handoff in 4G networks," in Electrical Engineering Computing Science and Automatic Control (CCE), 2010 7th International Conference on, 2010, pp. 309-314.

[62] J. R. Gallardo-Medina, U. Pineda-Rico and E. Stevens-Navarro, "VIKOR method for vertical handoff decision in beyond 3G wireless networks," in Electrical Engineering, Computing Science and Automatic Control,CCE,2009 6th International Conference on, 2009, pp. 1-5.

[63] Y. Wang, P. Zhang, Y. Zhou, J. Yuan, F. Liu and G. Li, "Handover Management in Enhanced MIH Framework for Heterogeneous Wireless Networks Environment," Wirel.Pers.Commun., vol. 52, pp. 615-636, February, 2010.

[64] P. N. Tran and N. Boukhatem, "Comparison of MADM decision algorithms for interface selection in heterogeneous wireless networks," in Software, Telecommunications and Computer Networks, 2008. SoftCOM 2008. 16th International Conference on, 2008, pp. 119-124.

[65] O. E. Falowo and H. A. Chan, "RAT selection for multiple calls in heterogeneous wireless networks using modified topsis group decision making technique," in Personal 
Indoor and Mobile Radio Communications (PIMRC), 2011 IEEE 22nd International Symposium on, 2011, pp. 1371-1375.

[66] I. Smaoui, F. Zarai, R. Bouallegue and L. Kamoun, "Multi-criteria dynamic access selection in heterogeneous wireless networks," in Wireless Communication Systems, 2009. ISWCS 2009. 6th International Symposium on, 2009, pp. 338-342.

[67] A. Sgora, D. D. Vergados and P. Chatzimisios, "An access network selection algorithm for heterogeneous wireless environments," in Computers and Communications (ISCC), 2010 IEEE Symposium on, 2010, pp. 890-892.

[68] L. Mohamed, C. Leghris and A. Adib, "A hybrid approach for network selection in heterogeneous multi-access environments," in New Technologies, Mobility and Security (NTMS), 2011 4th IFIP International Conference on, 2011, pp. 1-5.

[69] F. Bari and V. C. M. Leung, "Use of non-monotonic utility in multi-attribute network selection," in Wireless Telecommunications Symposium, 2007. WTS 2007, 2007, pp. 1-8.

[70] Liu Sheng-mei, Pan Su, Mi Zheng-kun, Meng Qing-min and Xu Ming-hai, "A simple additive weighting vertical handoff algorithm based on SINR and AHP for heterogeneous wireless networks," in Intelligent Computation Technology and Automation (ICICTA), 2010 International Conference on, 2010, pp. 347-350.

[71] Qingyang Song and A. Jamalipour, "A network selection mechanism for next generation networks," in Communications, 2005. ICC 2005. 2005 IEEE International Conference on, 2005, pp. 1418-1422 Vol. 2.

[72] Qingyang Song and A. Jamalipour, "Network selection in an integrated wireless LAN and UMTS environment using mathematical modeling and computing techniques," Wireless Communications, IEEE, vol. 12, pp. 42-48, 2005.

[73] Inwhee Joe, Won-Tae Kim and Seokjoon Hong, "A network selection algorithm considering power consumption in hybrid wireless networks," in Computer Communications and Networks, 2007. ICCCN 2007. Proceedings of 16th International Conference on, 2007, pp. 1240-1243.

[74] A. Ismail and Byeong-hee Roh, "Adaptive handovers in heterogeneous networks using fuzzy MADM," in Mobile IT Convergence (ICMIC), 2011 International Conference on, 2011, pp. 99-104.

[75] F. Bari and V. Leung, "Multi-attribute network selection by iterative TOPSIS for heterogeneous wireless access," in Consumer Communications and Networking Conference, 2007. CCNC 2007. 4th IEEE, 2007, pp. 808-812. 
[76] T. L. Saaty and L. G. Vargas, Models, Methods, Concepts \& Applications of the Analytic Hierarchy Process. Boston: Kluwer Academic Publishers, 2001.

[77] Zhao Song, Shi Wenxiao, Fan Shaoshua and Wang Nan, "A GRA-based network selection mechanism in heterogeneous wireless networks," in Computer, Mechatronics, Control and Electronic Engineering (CMCE), 2010 International Conference on, 2010, pp. 215-218.

[78] A. Huszák and S. Imre, "Eliminating rank reversal phenomenon in GRA-based network selection method," in Communications (ICC), 2010 IEEE International Conference on, 2010, pp. 1-6.

[79] Wang Yafang, Cui Huimin and Zhang Jinyan, "Network access selection algorithm based on the analytic hierarehy process and gray relation analysis," in New Trends in Information Science and Service Science (NISS), 2010 4th International Conference on, 2010, pp. 503-506.

[80] Tien-Chin Wang, Jia-Ling Liang and Chun-Yen Ho, "Multi-criteria decision analysis by using fuzzy VIKOR," in Service Systems and Service Management, 2006 International Conference on, 2006, pp. 901-906.

[81] S. Opricovic and G. Tzeng, "Compromise solution by MCDM methods: A comparative analysis of VIKOR and TOPSIS," Eur. J. Oper. Res., vol. 156, pp. 445-455, 7/16, 2004.

[82] Wenhui Zhang, "Handover decision using fuzzy MADM in heterogeneous networks," in Wireless Communications and Networking Conference, 2004. WCNC. 2004 IEEE, 2004, pp. 653-658 Vol.2.

[83] P. M. L. Chan, Y. F. Hu and R. E. Sheriff, "Implementation of fuzzy multiple objective decision making algorithm in a heterogeneous mobile environment," in Wireless Communications and Networking Conference, 2002. WCNC2002. 2002 IEEE, 2002, pp. 332-336 vol.1.

[84] Z. Yan, H. Luo, Y. Qin, H. Zhou, J. Guan and S. Zhang, "An adaptive multi-criteria vertical handover framework for heterogeneous networks," in Proceedings of the International Conference on Mobile Technology, Applications, and Systems, Yilan, Taiwan, 2008, pp. 14:1-14:7.

[85] P. Israt, N. Chakma and M. Hashem, "A fuzzy logic-based adaptive handoff management protocol for next-generation wireless systems," in Computer and Information Technology, 2008. ICCIT 2008. 11th International Conference on, 2008, pp. 288-293. 
[86] Ge Kun, Ji Hong and Li Xi, "A speed sensitive vertical handoff algorithm based on fuzzy control," in Wireless Communications, Networking and Mobile Computing, 2009. WiCom '09. 5th International Conference on, 2009, pp. 1-4.

[87] T. Thumthawatworn, A. Pervez and P. Santiprabhob, "Modular handover decision system based on fuzzy logic for wireless networks," in Electrical Engineering/Electronics, Computer, Telecommunications and Information Technology (ECTI-CON), 2011 8th International Conference on, 2011, pp. 385-388.

[88] Shih Jung Wu, "An intelligent handover decision mechanism for heterogeneous wireless networks," in Networked Computing and Advanced Information Management (NCM), 2010 Sixth International Conference on, 2010, pp. 688-693.

[89] K. Vasu, S. Maheshwari, S. Mahapatra and C. S. Kumar, "QoS aware fuzzy rule based vertical handoff decision algorithm for wireless heterogeneous networks," in Communications (NCC), 2011 National Conference on, 2011, pp. 1-5.

[90] Tao Yang and Peng Rong, "A fuzzy logic vertical handoff algorithm with motion trend decision," in Strategic Technology (IFOST), 2011 6th International Forum on, 2011, pp. 1280-1283.

[91] M. M. Alkhawlani, K. A. Alsalem and A. A. Hussein, "Multi-criteria vertical handover by TOPSIS and fuzzy logic," in Communications and Information Technology (ICCIT), 2011 International Conference on, 2011, pp. 96-102.

[92] X. Liu and L. Jiang, "A novel vertical handoff algorithm based on fuzzy logic in aid of grey prediction theory in wireless heterogeneous networks," Journal of Shanghai Jiaotong University (Science), vol. 17, pp. 25-30, 2012.

[93] Rong Chai, Jingjing Cheng, Xiansen Pu and Qianbin Chen, "Neural network based vertical handoff performance enhancement in heterogeneous wireless networks," in Wireless Communications, Networking and Mobile Computing (WiCOM), 2011 7th International Conference on, 2011, pp. 1-4.

[94] R. Suleesathira and S. Kunarak, "Neural network handoff in shadow-rayleigh fading," in Circuits and Systems, 2005. ISCAS 2005. IEEE International Symposium on, 2005, pp. 5146-5149 Vol. 5.

[95] S. Kunarak and R. Suleesathira, "Predictive RSS with fuzzy logic based vertical handoff algorithm in heterogeneous wireless networks," in Advanced Technologies for Communications (ATC), 2010 International Conference on, 2010, pp. 189-194.

[96] A. Çalhan and C. Çeken, "An adaptive neuro-fuzzy based vertical handoff decision algorithm for wireless heterogeneous networks," in Personal Indoor and Mobile Radio 
Communications (PIMRC), 2010 IEEE 21st International Symposium on, 2010, pp. 22712276.

[97] A. Çalhan and C. Çeken, "An Optimum Vertical Handoff Decision Algorithm Based on Adaptive Fuzzy Logic and Genetic Algorithm," Wireless Personal Communications, pp. 1-18, .

[98] A. Singhrova and N. Prakash, "Adaptive vertical handoff decision algorithm for wireless heterogeneous networks," in High Performance Computing and Communications, 2009. HPCC '09. 11th IEEE International Conference on, 2009, pp. 476-481.

[99] M. R. HeidariNezhad and Z. Zukarnain, "A Host Mobility Support with Adaptive Network Selection Method in Hybrid Wireless Environment," JDCTA, vol. 3, pp. 34-39, 2009.

[100] S. Altug, H. J. Trussell and Mo-Yuen Chow, "A "mutual update" training algorithm for fuzzy adaptive logic control/decision network (FALCON)," Neural Networks, IEEE Transactions on, vol. 10, pp. 196-199, 1999.

[101] M. Alkhawlani and A. Ayesh, "Access network selection based on fuzzy logic and genetic algorithms," Adv.in Artif.Intell., vol. 8, pp. 1:1-1:12, January, 2008.

[102] M. M. Alkhawlani and K. A. Alsalem, "Radio network selection for tight-coupled wireless networks," in Informatics and Systems (INFOS), 2010 the 7th International Conference on, 2010, pp. 1-8.

[103] S. Horrich, S. Ben Jamaa and P. Godlewski, "Neural networks for adaptive vertical handover decision," in Modeling and Optimization in Mobile, Ad Hoc and Wireless Networks and Workshops, 2007. WiOpt 2007. 5th International Symposium on, 2007, pp. $1-7$.

[104] Qiang Guo, Jie Zhu and Xianghua Xu, "An adaptive multi-criteria vertical handoff decision algorithm for radio heterogeneous network," in Communications, 2005. ICC 2005. 2005 IEEE International Conference on, 2005, pp. 2769-2773 Vol. 4.

[105] L. Giupponi, R. Agusti, J. Perez-Romero and O. Sallent, "A fuzzy neural JRRM in a heterogeneous scenario supported by prediction strategies for horizontal and vertical handovers," in Fuzzy Systems, 2006 IEEE International Conference on, 2006, pp. 655662.

[106] L. Giupponi, R. Agusti, J. Perez-Romero and O. Sallent Roig, "A Novel Approach for Joint Radio Resource Management Based on Fuzzy Neural Methodology," Vehicular Technology, IEEE Transactions on, vol. 57, pp. 1789-1805, 2008. 
[107] B. V. Quang, R. V. Prasad and I. Niemegeers, "A Survey on Handoffs - Lessons for $60 \mathrm{GHz}$ Based Wireless Systems," Communications Surveys \& Tutorials, IEEE, vol. 14, pp. 64-86, 2012.

[108] T. Oliveira, S. Mahadevan and D. P. Agrawal, "Handling network uncertainty in heterogeneous wireless networks," in INFOCOM, 2011 Proceedings IEEE, 2011, pp. 2390-2398.

[109] S. Dhar, A. Ray and R. Bera, "Design and Simulation of Vertical Handover Algorithm for Vehicular Communication," International Journal of Engineering Science and Technology, vol. 2, pp. 5509-5525, 2010.

[110] S. Dhar, A. Ray and R. Bera, "A Context Aware Vertical Handoff Algorithm for Vehicular Communication," International Journal of Electronics, Computer and Communications Technologies, vol. 2, pp. 24-34, 2011.

[111] A. Sgora, C. A. Gizelis and D. D. Vergados, "Network selection in a WiMAXWiFi environment," Pervasive and Mobile Computing, vol. 7, pp. 584-594, 2011.

[112] I. Chamodrakas and D. Martakos, "A utility-based fuzzy TOPSIS method for energy efficient network selection in heterogeneous wireless networks," Applied Soft Computing, vol. 11, pp. 3734-3743, 6, 2011.

[113] D. E. Charilas, O. I. Markaki, J. Psarras and P. Constantinou, "Application of Fuzzy AHP and ELECTRE to Network Selection," vol. 13, pp. 63-73, 2009.

[114] Y. Nkansah-Gyekye and J. I. Agbinya, "A vertical handoff decision algorithm for next generation wireless networks," in Broadband Communications, Information Technology \& Biomedical Applications, 2008 Third International Conference on, 2008, pp. 358-364.

[115] J. Rinne, "3GPP Specification Details, TS 23.107," .

[116] L. Mikhailov and P. Tsvetinov, "Evaluation of services using a fuzzy analytic hierarchy process," Applied Soft Computing, vol. 5, pp. 23-33, 12, 2004.

[117] M. T. Lamata, "Ranking of alternatives with ordered weighted averaging operators," Int J Intell Syst, vol. 19, pp. 473-482, May, 2004.

[118] C. Da-Yong, "Applications of the extent analysis method on fuzzy AHP," Eur. J. Oper. Res., vol. 95, pp. 649-655, 12/20, 1996.

[119] A. H. Gharehgozli, R. Tavakkoli-Moghaddam, M. Rabbani and N. Zaerpour, "Acceptance/rejection of incoming orders by a fuzzy analytical hierarchy process in make-to-order environments," in Proceedings of the 8th Conference on 8th WSEAS 
International Conference on Fuzzy Systems - Volume 8, Vancouver, Canada, 2007, pp. 202-207.

[120] H. Lin and W. Chang, "Order selection and pricing methods using flexible quantity and fuzzy approach for buyer evaluation," Eur. J. Oper. Res., vol. 187, pp. 415-428, 2008.

[121] D. Yong, "Plant location selection based on fuzzy TOPSIS," The International Journal of Advanced Manufacturing Technology, vol. 28, pp. 839-844, 2006.

[122] J. L. Deng, "Introduction to Grey system theory," J. Grey Syst., vol. 1, pp. 1-24, November, 1989.

[123] S. Saeed, C. Rezaei and B. H. Khalaj, "Grey Prediction Based Handoff Algorithm,".

[124] J. Lilly, "Takagi-sugeno fuzzy systems," in Fuzzy Control and IdentificationAnonymous Wiley-IEEE Press, 2010, pp. 88-105.

[125] E. H. Mamdani, "Application of fuzzy algorithms for control of simple dynamic plant," Electrical Engineers, Proceedings of the Institution of, vol. 121, pp. 1585-1588, 1974.

[126] E. Triantaphyllou, Multi-Criteria Decision Making Methods: A Comparative Study. 2000.

[127] T. Gwo-Hshiung and H. Jih-Jeng, Multiple Attribute Decision Making: Methods and Applications. 2011.

[128] C. Chen, C. Lin and S. Huang, "A fuzzy approach for supplier evaluation and selection in supply chain management," Int J Prod Econ, vol. 102, pp. 289-301, 8, 2006.

[129] C. Chen, "Extensions of the TOPSIS for group decision-making under fuzzy environment," Fuzzy Sets Syst., vol. 114, pp. 1-9, August, 2000.

[130] C. Cheng and Y. Lin, "Evaluating the best main battle tank using fuzzy decision theory with Linguistic criteria evaluation," Eur. J. Oper. Res., vol. 142, pp. 174, 2002.

[131] O. Serafim, "Fuzzy VIKOR with an application to water resources planning," Expert Syst. Appl., vol. 38, pp. 12983-12990, 9/15, 2011.

[132] Ruan Lian-fa and Chen Jialing, "Comparison and selection of green building programs based on fuzzy VIKOR method," in Consumer Electronics, Communications and Networks (CECNet), 2011 International Conference on, 2011, pp. 3027-3030. 
[133] C. Shan-Huo, "Ranking fuzzy numbers with maximizing set and minimizing set," Fuzzy Sets Syst., vol. 17, pp. 113-129, 11//, 1985.

[134] J. Zander and S. Kim, Radio Resource Management in Wireless Networks. Artech House, 2001.

[135] O. Queseth, "The effect of selfish behavior in mobile networks using CSMA/CA," in Vehicular Technology Conference, 2005. VTC 2005-Spring. 2005 IEEE 61st, 2005, pp. 2157-2161 Vol. 4.

[136] J. -. C. Wu and Hung-Huan Liu, "Performance evaluation for integrated voice/data wireless networks with link adaptation," in Vehicular Technology Conference, 2003. VTC 2003-Spring. the 57th IEEE Semiannual, 2003, pp. 1298-1302 vol.2.

[137] H. Liu, J. C. Wu and L. Lu, "The study of performance enhancement for integrated voice/data wireless networks by link adaptation," Comput. Commun., vol. 28, pp. 519528, March, 2005.

[138] D. Willkomm, S. Machiraju, J. Bolot and A. Wolisz, "Primary user behavior in cellular networks and implications for dynamic spectrum access," Communications Magazine, IEEE, vol. 47, pp. 88-95, 2009.

[139] M. Hata, "Empirical formula for propagation loss in land mobile radio services," Vehicular Technology, IEEE Transactions on, vol. 29, pp. 317-325, 1980.

[140] T. Rappaport, Wireless Communciations: Principles and Practice. Prentice Hall, 2002. 
VITA

\section{FAISAL KALEEM}

Born, Karachi, Pakistan

1989-1993

B.S., Electrical Engineering

N.E.D. University of Engineering \& Technology

Karachi, Pakistan

1996-1999

M.S., Electrical Engineering

Florida International University

Miami, Florida

$1998-2000$

Instructor

School of Computer Science

Florida International University

Miami, Florida

2000-2010

Instructor

DSIS, College of Business

Florida International University

Miami, Florida

2000-till date

Certified Trainer

Florida International University

Miami, Florida

$2004-2012$

Doctoral Candidate

Florida International University

Miami, Florida

2008-2008

Faculty Award for Excellence in Teaching

Florida International University

Miami, Florida

2010-till date

Instructor

College of Engineering

Florida International University

Miami, Florida

\section{PUBLICATIONS AND PRESENTATIONS}

K. Yen, F. Kaleem, J. Andrian, P. Schmidt, "Secure Communication with Enhanced Steganography," Signal Processing, Communications \& Computer Science, edited by Nikos Mastoralcis, pp.90-96, World Scientific, 2000

K. Yen, F. Kaleem, "Encryption Enhancement of Steganography with Chaotic Signals," Information: An Int'1 Journal, vol. 4, no.3, pp.327-334, 2001 
F. Kaleem, K. Yen, A. Caballero, "Intelligent Spatial Filters Based on Fuzzy Logic," WSEAS Trans. on Circuits, vol.2, no.1, pp.252-257, 2003

F. Kaleem, K. K. Yen, A. Caballero, "Java Program Implementation of HDLC Protocol for Serial Data Communication," World Trans. on Engineering \& Technology Education, vol.2, no.2, pp.331-349, 2003

K. Yen, F. Kaleem, "Implementation of HDLC Based Serial Data Communication Using Java," Proc. of 2003 Int'l Conf. on Communications in Computing (CIC'03), June 23-26, 2003

F. Kaleem, K.K. Yen, A.A. Caballero, "Java Program Implementation of HDLC Protocol For Series Data Communication," World Trans. on Engineering \& Technology Education, vol. 2, no.2, pp.331-334, 2003

F. Kaleem, "Online = An Easy 'A', Dispelling the Myth”, FIU ONLINE Conference, March 2008

F. Kaleem, "Professors, Beware!", FIU Faculty Senate Technology Committee Conference , April 2009

F. Kaleem, "RFID Security", video presentation for NBC Channel 6, Miami-Florida, February 2011

F. Kaleem, "Smartphone Security", video presentation for NBC Channel 6, Miami-Florida, February 2011

F. Kaleem, "Smartphone Security", video presentation for CBS Channel 12, West Palm BeachFlorida, April 2011

F. Kaleem, K. K. Yen, "Wireless Security”, Information Assurance and Security Technologies for Risk Assessment and Threat Management: Advances, pp. 17-45, 2012

HONORS AND AWARDS

2000

2002

2010

May 2002

December 2004

December 2004

August 2006

December 2006

December 2006

August 2007

August 2007

December 2007

August 2008

December 2008

August 2009

December 2010
Induction in Tau Beta Pi Honor Society

Induction in Eta Kappa Nu Honor Society

Induction in Golden Key International Honor Society

Best Professor Award

Best Course (Information Security) Award

Best Professor Award

Best Course (Information Security) Award

Best Course (Information Security) Award

Best Professor Award

Best Professor Award

Best Course (Information Security) Award

Best Professor Award

Best Professor Award

Best Professor Award

Best Professor Award

Best Professor Award 